\title{
On Maximum-Sized Golden-Mean Matroids
}

Michael Welsh

\section{VICTORIA UNIVERSITY OF WELLINGTON}

Te Whare Wananga o te Upoko o te Ika a Maui

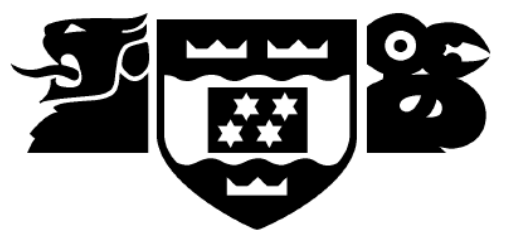

School of Mathematics, Statistics and Operations Research

Te Kura Matai Tatauranga, Rangahau Punaha

A thesis

submitted to the Victoria University of Wellington in fulfilment of the requirements for the degree of

Doctor of Philosophy in Mathematics.

Victoria University of Wellington 



\begin{abstract}
A rank- $r$ simple matroid is maximum-sized in a class if it has the largest number of elements out of all simple rank- $r$ matroids in that class. Maximumsized matroids have been classified for various classes of matroids: regular (Heller, 1957); dyadic (Kung and Oxley, 1988-90); k-regular (Semple, 1998); near-regular and sixth-root-of-unity (Oxley, Vertigan, and Whittle, 1998). Golden-mean matroids are matroids that are representable over the goldenmean partial field. Equivalently, a golden-mean matroid is a matroid that is representable over $G F(4)$ and $G F(5)$.

Archer conjectured that there are three families of maximum-sized goldenmean matroids. This means that a proof of Archer's conjecture is likely to be significantly more complex than the proofs of existing maximum-sized characterisations, as they all have only one family.

In this thesis, we consider the four following subclasses of golden-mean matroids: those that are lifts of regular matroids, those that are lifts of nearregular matroids, those that are golden-mean-graphic, and those that have a spanning clique. We close each of these classes under minors, and prove that Archer's conjecture holds in each of them.

It is anticipated that the last of our theorems will lead to a proof of Archer's conjecture for golden-mean matroids of sufficiently high rank.
\end{abstract}





\section{Acknowledgements}

I would like to thank Dillon Mayhew for his advice and supervision. I am also grateful to Steven Archer, Ben Clark, Peter Nelson, Rudi Pendavingh, Stefan van Zwam, and Geoff Whittle for various contributions to the research undertaken in this thesis.

Thanks go to Amanda Cameron and Susan Jowett for proof-readng parts of this thesis.

Lastly, I would like to thank my wife, Melissa, in particular for her naming the forbidden configurations in Chapter 6. 



\section{Contents}

1 Introduction $\quad 1$

1.1 History . . . . . . . . . . . . . . . . . 2

1.2 Golden-mean .................. . . 6

2 Preliminaries $\quad 11$

2.1 Sage ............................ 11

2.1.1 Partial Fields . . . . . . . . . . . . . . . 11

2.1.2 The Lift Theorem . . . . . . . . . . . . . . . . 13

$2.1 .3 G F(4) \ldots \ldots \ldots \ldots \ldots \ldots$

2.2 The Three Families . . . . . . . . . . . . . . . . . . . 15

2.2.1 G-matrices................... 15

$2.2 .2 G F(4)$ Matrices . . . . . . . . . . . . . 16

2.2.3 Minors . . . . . . . . . . . . . . . . 16

2.2.4 G-graphic representations . . . . . . . . 30

2.2.5 Augmented Cliques . . . . . . . . . . . . . 33

2.3 Rank Three ..................... 33

2.4 2-rounded Sets . . . . . . . . . . . . . . . 35

2.A Code .......................... 36

2.A.1 Universal Variables . . . . . . . . . . . . 36

2.A.2 Universal Functions . . . . . . . . . . . . 37 
2.A.3 Lemma 2.1.11 . . . . . . . . . . . . . . 37

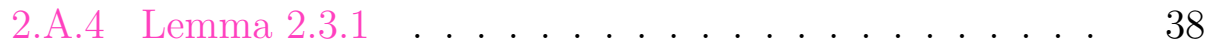

2.A.5 Lemma $2.4 .3 \ldots \ldots . \ldots . \ldots . \ldots$

3 Connectivity 43

3.13 -connectivity . . . . . . . . . . . . . . . 43

3.2 Vertical Connectivity . . . . . . . . . . . . 46

4 Lifts of Regular and Near-regular $\quad 57$

4.1 Preliminaries ..................... 57

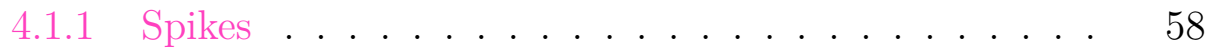

4.1 .2 Small Circuits . . . . . . . . . . . 60

$4.1 .3 \mathcal{L}(M, e) \ldots \ldots \ldots \ldots \ldots$

4.1 .4 Deficit Functions . . . . . . . . . . . 66

4.2 Lifts of Regular . . . . . . . . . . . . . . . . 71

4.3 Lifts of Near-regular . . . . . . . . . . . . . 76

4.3.1 All coloops . . . . . . . . . . . . 89

4.3.2 $M\left(K_{2, r-2}^{+}\right) \ldots \ldots \ldots \ldots \ldots \ldots$

4.4 A Strategic Hurdle . . . . . . . . . . . . . . . . . 100

4.A Code......................... 101

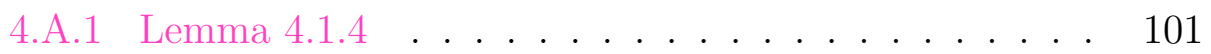

4.A.2 Lemma 4.1.5 . . . . . . . . . . . . . . . . . 102

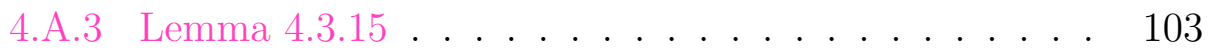

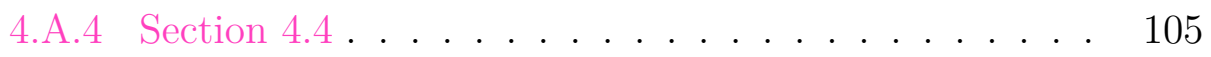

5 G-graphic Matroids 109

5.1 Preliminaries . . . . . . . . . . . . . . . . 109

5.1 .1 Graphs With No $K_{4} \ldots \ldots \ldots 111$

5.2 Results.......................... . . . 113 
5.A Code.......................... 118

5.A.1 Lemma 5.2.3 . . . . . . . . . . . . . . . . 118

5.A.2 Sublemma 5.2.4.1 . . . . . . . . . . . . . 119

5.A.3 Sublemma 5.2.4.2 . . . . . . . . . . . 122

6 Matroids with a Spanning Clique 125

6.1 Preliminaries ..................... 125

6.2 Red-Green Lemma . . . . . . . . . . . . . . . . . . . . . 127

6.3 Forbidden Configurations . . . . . . . . . . . . . . 129

6.3.1 Complete Graphs . . . . . . . . . . . . . . . 131

6.3.2 Forbidden Configurations . . . . . . . . . . . 131

6.4 Non-clique Three-point Lines . . . . . . . . . . . . . . . 136

6.4.1 One Non-clique Three-point Line . . . . . . . . . . . 139

6.4.2 Two Non-clique Three-point Lines . . . . . . . . . . . 147

6.4.3 Three Non-clique Three-point Lines . . . . . . . . . . 158

$6.5 U_{2,4}$-restrictions . . . . . . . . . . . . . . . 170

6.5.1 One Non-clique Element . . . . . . . . . . . . . 170

6.5.2 Two Non-clique Elements . . . . . . . . . . . . . 171

6.5.3 Three Non-clique Elements . . . . . . . . . . . . . . 171

6.6 Proof of Theorem 6.1.4 . . . . . . . . . . . . . . . 190

6.7 Future Work . . . . . . . . . . . . . . . . . . . 217

6.A Code.......................... 218

6.A.1 Lemma 6.1.5 . . . . . . . . . . . . . 218

6.A.2 Lemma 6.2.1 . . . . . . . . . . . . . 218

6.A.3 Testing For Forbidden Configurations . . . . . . . . . 219

6.A.4 Finding Lines . . . . . . . . . . . . . . . . . . . . . . 219

6.A.5 Finding Multiple Lines . . . . . . . . . . . . . . 221

6.A.6 Testing For Betsy Ross . . . . . . . . . . . . . . . 222 
6.A.7 Sublemma 6.6.19.1 . . . . . . . . . . . . . 223

$\begin{array}{ll}\text { Bibliography } & 228\end{array}$

Index

232 


\section{List of Figures}

2.1 G-matrices of the golden-mean families . . . . . . . . . 17

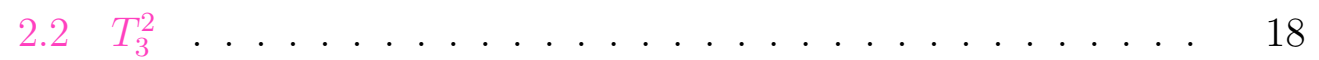

$2.3 \quad G_{3} \ldots \ldots \ldots \ldots \ldots \ldots$

$2.4 H P_{3} \ldots \ldots \ldots \ldots \ldots \ldots \ldots$

$2.5 G F(4)$ matrices of the golden-mean families . . . . . . . . 19

2.6 Pivoted GF(4)-matrices of $T_{r}^{2}$ for Sublemma 2.2.1.1 . . . . 21

2.7 Pivoted $G F(4)$-matrices of $G_{r}$ for Sublemma 2.2.1.2 I . . . . 23

2.8 Pivoted $G F(4)$-matrices of $G_{r}$ for Sublemma 2.2.1.2 II . . . 25

2.9 Pivoted GF(4)-matrices of $G_{r}$ for Sublemma 2.2.1.2 III . . . 26

2.10 Pivoted GF(4)-matrices of $H P_{r}$ for Sublemma 2.2.1.3 I . . . 28

2.11 Pivoted GF(4)-matrices of $H P_{r}$ for Sublemma 2.2.1.3 II . 29

2.12 Pivoted GF(4)-matrices of $H P_{r}$ for Sublemma 2.2.1.3 III . . 31

$2.13 \mathbb{G}$-graphic representation of $T_{4}^{2} \ldots \ldots 32$

$2.14 \mathbb{G}$-graphic representation of $G_{4} \ldots \ldots 32$

2.15 Augmented clique representations of all members of $\mathscr{G}_{3} \ldots 33$

2.16 The Betsy Ross $(B R) \ldots \ldots . \ldots . \ldots 34$

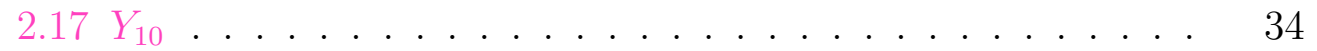

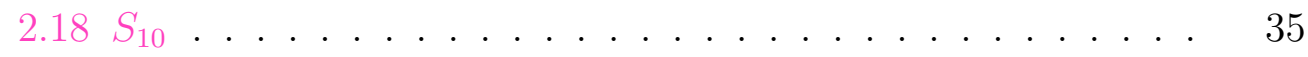

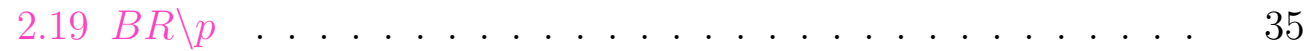

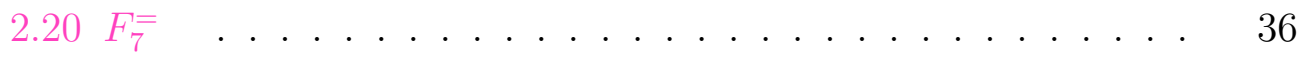


$2.21 Q_{6} \ldots \ldots \ldots \ldots \ldots \ldots \ldots$

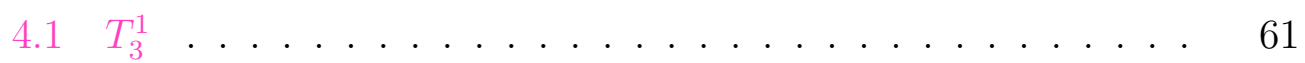

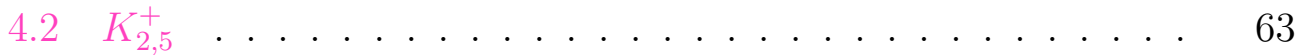

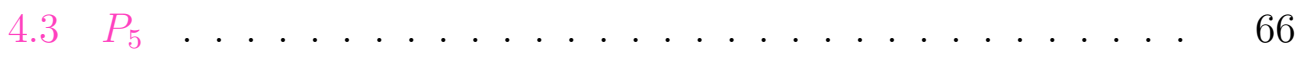

4.4 Forbidden Configuration from Lemma 4.2.5 . . . . . . . . 73

4.5 Three triangles of $T_{3}^{1}$ for Lemma $4.3 .13 \ldots \ldots$. . . . . 84

4.6 Outcomes (ii) and (iii) of Lemma 4.3.14 . . . . . . . . . 84

$4.7 G F(4)$ matrices for Lemma $4.3 .23 \ldots \ldots 92$

4.8 GF(4) matrices for Lemma 4.3.28 . . . . . . . . . . . . 97

4.9 GF(4) matrices for the proof of Theorem 4.1.2 . . . . . . 99

5.1 From left to right: the theta, barbell, and handcuff graphs . 110

$5.2 \mathbb{G}$-graphic representation of $U_{2,5} \ldots \ldots \ldots$

5.3 The graph $2 K_{4}^{\circ} \ldots \ldots \ldots \ldots$

5.4 Labels for Lemma 5.2.5 . . . . . . . . . . . . . . . . . 115

5.5 Labels for Lemma 5.2.6 . . . . . . . . . . . . . 116

$6.1 F_{7}=$ for Lemma $6.3 .2 \ldots \ldots \ldots \ldots \ldots$

6.2 Edge Labels for $K_{4} \ldots \ldots \ldots$

6.3 Edge Labels for $K_{5} \ldots \ldots \ldots$

6.4 Edge Labels for $K_{6} \ldots \ldots \ldots$

6.5 Edge Labels for $K_{7} \ldots \ldots \ldots$

6.6 Edge Labels for $K_{8} \ldots \ldots \ldots$

6.7 Forbidden configurations with one green triangle and one red matching . . . . . . . . . . . . . . 135

6.8 Forbidden configurations with only green triangles . . . . . 137

6.9 Forbidden configurations with two red matchings . . . . . 138

6.10 Forbidden configurations with three red matchings I . . . . . 139

6.11 Forbidden configurations with three red matchings II . . . . 140 
6.12 Remaining forbidden configurations I . . . . . . . . . . 141

6.13 Remaining forbidden configurations II . . . . . . . . . . . . 142

6.14 Two Green Triangles . . . . . . . . . . . . . . . . . . . . . 142

6.15 Two Red Matchings . . . . . . . . . . . . . . . . . . . . 144

6.16 One Green Triangle and One Red Matching . . . . . . . . . 145

6.17 One Non-clique Three-point line . . . . . . . . . . . . . . . . 146

6.18 Starting from a GG I . . . . . . . . . . . . . . . . . 148

6.19 Starting from a GG II . . . . . . . . . . . . . . . . . 150

6.20 Starting from a GR . . . . . . . . . . . . . . 153

6.21 Starting from a RR-square . . . . . . . . . . . . . . . 155

6.22 Starting from a RR-digon . . . . . . . . . . . . . 157

6.23 Starting from $e$ in a red matching . . . . . . . . . . . . 158

6.24 Two Non-clique Three-point Lines . . . . . . . . . . . . . . . 159

6.25 Starting from a $\Gamma \ldots \ldots \ldots$. . . . . . . . . 160

6.26 Starting from a $\Delta \ldots \ldots \ldots$. . . . . . . . . . 162

6.27 Starting from a $\Theta \ldots \ldots . \ldots \ldots$. . . . . . . . . 163

6.28 Starting from a $\Lambda \ldots \ldots$. . . . . . . . . . . 164

6.29 Starting from a $\Pi \ldots \ldots \ldots \ldots$. . . . . . . . . . . 165

6.30 Starting from a $\Upsilon \ldots \ldots \ldots$. . . . . . . . . . 166

6.31 Starting from a $\Phi \ldots \ldots$. . . . . . . . . . . 167

6.32 Starting from a $\Psi \ldots \ldots$. . . . . . . . . . . . 168

6.33 Starting from a $\Omega \ldots \ldots \ldots$. . . . . . . . . . 169

6.34 Three non-clique three-point lines . . . . . . . . . . . . 170

6.35 Three Green Triangles . . . . . . . . . . . . . . . . . 172

6.36 Two Green Triangles and One Red Matching . . . . . . . . . 175

6.37 One Green Triangle and Two Red Matchings I . . . . . . . . 177

6.38 One Green Triangle and Two Red Matchings II . . . . . . . 178

6.39 Three Red Matchings I . . . . . . . . . . . . . . . . . . . . . 181 
6.40 Three Red Matchings II . . . . . . . . . . . . . . . . . . 182

6.41 Three Red Matchings III . . . . . . . . . . . . . . . . . 183

6.42 Three Red Matchings IV . . . . . . . . . . . . . . . . . 184

$6.43 U_{2,4}$-restrictions . . . . . . . . . . . . . . . . . . 190

6.44 Line-star configuration . . . . . . . . . . . . . . . 194

6.45 Two-parallel configuration . . . . . . . . . . . . 195

6.46 Matching-star configuration . . . . . . . . . . . . 199

6.47 Betsy Ross configuration . . . . . . . . . . . . . . . 203

6.48 Five-point line with four non-clique elements . . . . . . . . . 207

6.49 Schematics of the four possible outcomes for $C_{e}$ and $C_{f} \ldots 212$ 


\section{List of Tables}

1.1 Values of $h(r)$ for $r \in\{2, \ldots, 7\} \ldots \ldots . \ldots$

2.1 Values of $\psi$, the unique homomorphism from $\mathbb{G}$ to $G F(19)$ such that $\psi(\tau)=5 \ldots \ldots \ldots \ldots \ldots$

6.1 How the eleven ways to get $e$ on two non-clique three-point lines (Figure 6.24) arise from the five ways to have $e$ on one non-clique three-point line (Figure 6.17 and $e$ being in a red matching) . . . . . . . . . . . . . . . . 158 
Lying in bed would be an altogether perfect and supreme experience if only one had a coloured pencil long enough to draw on the ceiling.

G.K. Chesterton

\section{1 \\ Introduction}

We start with a definition.

Definition 1.0.1. Let $\mathcal{M}$ be a minor-closed class of matroids and let $r$ be a non-negative integer. The growth-rate function of $\mathcal{M}$ is

$$
h_{\mathcal{M}}(r)=\max \{\epsilon(M) \mid M \in \mathcal{M} \text { and } r(M) \leq r\} \text {, }
$$

where $\epsilon(M)$ is the number of rank-one flats of $M$.

We say that $M$ is maximum-sized in $\mathcal{M}$ if $M$ is a simple matroid in $\mathcal{M}$ such that $\epsilon(M)=h_{\mathcal{M}}(r(M))$.

The results in this thesis characterise the matroids that maximise the growthrate function for various classes of golden-mean matroids. For an overview of growth-rate functions, see Section 14.10 in Oxley [10]. 


\section{$1.1 \quad$ History}

It is easy to characterise the maximum-sized matroids in the classes of graphic matroids and matroids representable over a finite field. The next two results are well-known.

Theorem 1.1.1. Let $\mathcal{M}$ be the class of graphic matroids. Then

$$
h_{\mathcal{M}}(r)=\left(\begin{array}{c}
r+1 \\
2
\end{array}\right)
$$

Furthermore, $M \in \mathcal{M}$ is maximum-sized if and only if $M$ is the cycle matroid of a complete graph.

Theorem 1.1.2. Let $\mathcal{M}_{q}$ be the class of matroids representable over the finite field $G F(q)$. Then

$$
h_{\mathcal{M}_{q}}(r)=\frac{q^{r}-1}{q-1}
$$

Furthermore, $M \in \mathcal{M}_{q}$ is maximum-sized if and only if $M$ is the rank-r $(M)$ projective geometry over $G F(q)$.

Other natural classes of matroids arise when we consider the intersection of two or more finite fields. In order to best understand these classes, we use partial fields.

Partial fields were introduced by Semple and Whittle [17]. However, we will follow the treatment of Pendavingh and Van Zwam [12], starting from a ring. For a more thorough introduction to partial fields, see Pendavingh and Van Zwam [12, 13].

Definition 1.1.3. A partial field is a pair $(R, G)$, where $R$ is a commutative ring with identity, and $G$ is a subgroup of the group of units of $R$ such that $-1 \in G$.

If $S$ is a subset of elements of some group, then $\langle S\rangle$ is the subgroup generated by $S$. If $S$ is a subset of non-zero elements of a ring, then $\langle S\rangle$ denotes the multiplicative subgroup generated by $S$. The group of elements with a multiplicative inverse in a ring $R$ is denoted by $R^{*}$. If $R$ is a ring and $S$ a set of symbols, then we denote the polynomial ring over $R$ on $S$ by $R[S]$. 
Definition 1.1.4. If $\mathbb{P}=(R, G)$ is a partial field, and $p \in R$, then we say that $p$ is an element of $\mathbb{P}$ (notation: $p \in \mathbb{P}$ ) if $p=0$ or $p \in G$. We define $\mathbb{P}^{*}$ to be $G$.

Definition 1.1.5. A matroid $M$ is said to be representable over the partial field $\mathbb{P}$ if there is a matrix $\mathfrak{M}$ such that all non-zero subdeterminants of $\mathfrak{M}$ are in $\mathbb{P}$ and a labelling of the columns of $\mathfrak{M}$ by $E(M)$ such that any subset $\left\{x_{1}, \ldots, x_{k}\right\}$ is independent in $M$ if and only if the submatrix $\left[x_{1}, \ldots, x_{k}\right]$ contains a $k \times k$ subdeterminant that is non-zero in $\mathbb{P}$. We say that $\mathfrak{M}$ is a $\mathbb{P}$-matrix, and that $M$ is a $\mathbb{P}$-matroid.

Note that every field $\mathbb{F}$ can be considered as the partial field $\left(\mathbb{F}, \mathbb{F}^{*}\right)$.

The following result, which shows why we are interested in partial fields, is an unpublished result of Vertigan that appears in Whittle [24]. A stronger result is given by Pendavingh and Van Zwam [13, Corollary 2.20].

Lemma 1.1.6. If $\mathcal{F}$ is any set of fields, then, for some partial field $\mathbb{P}$, the class of matroids representable over all fields in $\mathcal{F}$ is the class of $\mathbb{P}$ representable matroids.

Maximum-sized matroids have been characterised for various partial fields. We give some examples here. For a catalogue of partial fields, see Pendavingh and Van Zwam [12, Appendix A].

The first partial field we consider is the regular partial field.

Definition 1.1.7. The regular partial field is the partial field

$$
\mathbb{U}_{0}=(\mathbb{Z},\{-1,0,1\})
$$

A $\mathbb{U}_{0}$-matroid is said to be regular.

Theorem 1.1.8 (Tutte, in Oxley [10, Theorem 6.6.3]). The following are equivalent for a matroid $M$ :

(i) $M$ is regular.

(ii) $M$ is representable over every field.

(iii) $M$ is binary and, for some field $\mathbb{F}$ of characteristic other than two, $M$ is $\mathbb{F}$-representable. 
Theorem 1.1.9 (Heller [6]). Let $\mathfrak{U}_{0}$ be the class of matroids representable over $\mathbb{U}_{0}$. Then

$$
h_{\mathcal{U}_{0}}(r)=\left(\begin{array}{c}
r+1 \\
2
\end{array}\right)
$$

Furthermore, $M \in \mathcal{U}_{0}$ is maximum-sized if and only if $M \cong M\left(K_{r(M)+1}\right)$.

Next, we consider the near-regular partial field.

Definition 1.1.10. The near-regular partial field is the partial field

$$
\mathbb{U}_{1}=\left(\mathbb{Z}\left[\beta, \frac{1}{1-\beta}, \frac{1}{\beta}\right],\langle-1, \beta, 1-\beta\rangle\right),
$$

where $\beta$ is an indeterminate. A $\mathbb{U}_{1}$-matroid is said to be near-regular.

Theorem 1.1.11 (Whittle [23, Theorem 1.4]). The following statements are equivalent for a matroid $M$ :

(i) $M$ is near-regular.

(ii) $M$ is representable over $G F(3)$ and $G F(8)$.

(iii) $M$ is representable over $G F(3), G F(4)$, and $G F(5)$.

(iv) $M$ is representable over $G F(3), G F(4)$, and $\mathbb{Q}$.

(v) $M$ is representable over all fields except possibly $G F(2)$.

Theorem 1.1.12 (Oxley, Vertigan, and Whittle [11, Corollary 2.2]). Let $\mathcal{U}_{1}$ be the class of matroids representable over $\mathbb{U}_{1}$. Then

$$
h_{\mathfrak{U}_{1}}(r)=\left(\begin{array}{c}
r+2 \\
2
\end{array}\right)-2
$$

Furthermore, $M \in \mathcal{U}_{1}$ is maximum-sized if and only if $M \cong T_{r(M)}^{1}$.

The matroid $T_{r}^{1}$ will be defined in Section 4.1.2.

The regular and near-regular partial fields can be generalised to give the $k$-regular partial field.

Definition 1.1.13. The $\boldsymbol{k}$-regular partial field is the partial field

$$
\mathbb{U}_{k}=\left(\mathbb{Q}\left(\alpha_{1}, \ldots, \alpha_{k}\right),\left\langle\left\{x-y \mid x, y \in\left\{0,1, \alpha_{1}, \ldots, \alpha_{k}\right\}, x \neq y\right\}\right\rangle\right),
$$

where $\alpha_{1}, \ldots, \alpha_{k}$ are indeterminates. $\mathrm{A} \mathbb{U}_{k}$-matroid is said to be $k$-regular. 
Let $D_{n}$ denote the $n \times\left(\begin{array}{l}n \\ 2\end{array}\right)$ matrix whose columns consist of all $n$-tuples with two non-zero entries, with the first being 1 and the second being -1 . The matroid $T_{r}^{k}$ is represented over $\mathbb{U}_{k}$ by the following matrix.

$\left[\begin{array}{c|c|c|c|c|c|c}1 & 0 \cdots & 1 \cdots 1 & \alpha_{1} \cdots \alpha_{1} & \cdots & \alpha_{k} \cdots \alpha_{k} & 0 \cdots 0 \\ \hline 0 & & & & & & \\ \vdots & I_{r-1} & I_{r-1} & I_{r-1} & \cdots & I_{r-1} & D_{r-1} \\ 0 & & & & & & \end{array}\right]$.

Theorem 1.1.14 (Semple [16, Theorem 2.3]). Let $\mathfrak{U}_{k}$ be the class of matroids representable over $\mathbb{U}_{k}$. Then

$$
h_{\mathfrak{U}_{k}}(r)=\left(\begin{array}{c}
r+k+1 \\
2
\end{array}\right)-\frac{k(k+3)}{2} .
$$

Furthermore, if $r \neq 3$ or $k \neq 2$, then $M \in \mathcal{U}_{k}$ is maximum-sized if and only if $M \cong T_{r(M)}^{k}$. If $r=3$ and $k=2$, then $M \in \mathcal{U}_{k}$ is maximum-sized if and only if $M$ is isomorphic to either $T_{3}^{2}$ (Figure 2.2) or $S_{10}$ (Figure 2.18).

Now we consider the sixth-roots-of-unity partial field.

Definition 1.1.15. The sixth-roots-of-unity partial field is the partial field

$$
\mathbb{S}=(\mathbb{Z}[\zeta],\langle\zeta\rangle)
$$

where $\zeta$ is a root of $x^{2}-x+1=0$. A $\mathbb{S}$-matroid is said to be a sixth-rootsof-unity matroid.

Theorem 1.1.16 (Whittle [23, Theorem 1.2]). The following statements are equivalent for a matroid $M$ :

(i) $M$ is a sixth-roots-of-unity matroid.

(ii) $M$ is representable over $G F(3)$ and $G F(4)$.

(iii) $M$ is representable over $G F(3)$ and $G F\left(2^{k}\right)$ for some even integer $k$.

Theorem 1.1.17 (Oxley, Vertigan, and Whittle [11, Theorom 2.1]). Let $\mathcal{S}$ be the class of matroids representable over $\mathbb{S}$. Then

$$
h_{\mathcal{S}}(r)= \begin{cases}\left(\begin{array}{c}
r+2 \\
2
\end{array}\right)-2 & \text { if } r \neq 3 \\
9 & \text { if } r=3\end{cases}
$$


Furthermore, $M \in \mathcal{S}$ is maximum-sized if and only if $M \cong T_{r(M)}^{1}$ when $r(M) \neq 3$, or $M \cong A G(2,3)$ when $r(M)=3$.

Finally, we consider the dyadic partial field.

Definition 1.1.18. The dyadic partial field is the partial field

$$
\mathbb{D}=\left(\mathbb{Z}\left[\frac{1}{2}\right],\langle-1,2\rangle\right)
$$

A $\mathbb{D}$-matroid is said to be dyadic.

Theorem 1.1.19 (Whittle [23, Theorem 1.1]). The following statements are equivalent for a matroid $M$ :

(i) $M$ is dyadic.

(ii) $M$ is representable over $G F(3)$ and $G F(5)$.

(iii) $M$ is representable over $G F(p)$ for all odd primes $p$.

(iv) $M$ is representable over $G F(3)$ and $\mathbb{Q}$.

(v) $M$ is representable over $G F(3)$ and $\mathbb{R}$.

(vi) $M$ is representable over $G F(3)$ and $G F(q)$ where $q$ is an odd prime power such that $q \equiv 2(\bmod 3)$.

The matroid $Q_{r}\left(G F(3)^{*}\right)$ is represented over $G F(3)$ by the matrix $\left[I_{r} \mid H_{r}\right]$, where $H_{r}$ is the ternary matrix whose columns consist of all $r$-tuples with two non-zero entries, with the first being 1 .

The next theorem follows from work done by Kung [8] and Kung and Oxley [9].

Theorem 1.1.20. Let $\mathcal{D}$ be the class of matroids representable over $\mathbb{D}$. Then

$$
h_{\mathcal{D}}(r)=r^{2}
$$

Furthermore, $M \in \mathcal{D}$ is maximum-sized if and only if $M \cong Q_{r(M)}\left(G F(3)^{*}\right)$.

\subsection{Golden-mean}

Definition 1.2.1. The golden-mean partial field is the partial field

$$
\mathbb{G}=(\mathbb{Z}[\tau],\langle-1, \tau\rangle)
$$


where $\tau$ is the positive root of $x^{2}-x-1$.

A matroid is golden-mean if it has a $\mathbb{G}$-representation.

The following theorem is an unpublished result of Vertigan. In his masters thesis, Semple [15] proved that (ii) implies (iii). For a proof, see Pendavingh and Van Zwam [13, Theorem 1.3].

Theorem 1.2.2. Let $M$ be a matroid. The following are equivalent:

(i) $M$ is representable over both $G F(4)$ and $G F(5)$;

(ii) $M$ is golden-mean;

(iii) $M$ is representable over $G F(p)$ for all primes $p$ such that $p=5$ or $p \equiv \pm 1(\bmod 5)$, and also over $G F\left(p^{2}\right)$ for all primes $p$.

The following conjecture is the subject of this thesis. It was stated by Archer in his $\mathrm{PhD}$ thesis [1].

Conjecture 1.2.3. Let $\mathcal{G}$ be the class of matroids representable over $\mathbb{G}$. Then

$$
h_{\mathcal{G}}(r)= \begin{cases}\left(\begin{array}{c}
r+3 \\
2
\end{array}\right)-5 & \text { if } r \neq 3 \\
11 & \text { if } r=3 .\end{cases}
$$

Furthermore, $M \in \mathcal{G}$ is maximum-sized if and only if $M$ is isomorphic to a member of $\mathscr{G}_{r(M)}$ when $r(M) \neq 3$, or $M$ is isomorphic to the Betsy Ross when $r(M)=3$.

The set $\mathscr{G}_{r}$ contains three matroids, $T_{r}^{2}, G_{r}$, and $H P_{r}$. These three matroids are described in Section 2.2. The Betsy Ross matroid, or $B R$, will be described in Section 2.3.

In his thesis, Archer proved Conjecture 1.2.3 for ranks three and four using a computer search.

From now on, the function $h(r)$ will be the function $h_{\mathcal{G}}(r)$ from Conjecture 1.2.3. In Table 1.1 we show some values for $h(r)$.

While we do not prove Conjecture 1.2.3 in this thesis, we do prove the conjecture for various subclasses of golden-mean matroids.

In Chapter 2, we introduce some important concepts that will be used throughout the thesis, and in Chapter 3 we prove some connectivity conditions. 


\begin{tabular}{c|c}
$r$ & $h(r)$ \\
\hline 2 & 5 \\
3 & 11 \\
4 & 16 \\
5 & 23 \\
6 & 31 \\
7 & 40
\end{tabular}

Table 1.1: Values of $h(r)$ for $r \in\{2, \ldots, 7\}$

The first two subclasses we prove Conjecture 1.2 .3 for are $\mathcal{R}$ and $\mathcal{N}$.

Let $\mathcal{M}_{1}$ be the set of golden-mean matroids $M$ with the property that $M / e$ is regular for some $e \in E(M)$. Let $\mathcal{R}$ be the set of all minors of matroids in $\mathcal{M}_{1}$.

Theorem 4.1.1. Let $\mathcal{R}$ be the class of matroids defined above. Then

$$
h_{\mathcal{R}}(r)=\left(\begin{array}{c}
r+3 \\
2
\end{array}\right)-5 .
$$

Furthermore, $M \in \mathcal{R}$ is maximum-sized if and only if $M \cong T_{r(M)}^{2}$.

Let $\mathcal{M}_{2}$ be the set of golden-mean matroids $M$ with the property that $M / e$ is near-regular for some $e \in E(M)$. Let $\mathcal{N}$ be the set of all minors of matroids in $\mathcal{M}_{2}$.

Theorem 4.1.2. Let $\mathcal{N}$ be the class of matroids defined above. Then

$$
h_{\mathcal{N}}(r)=\left(\begin{array}{c}
r+3 \\
2
\end{array}\right)-5
$$

Furthermore, $M \in \mathcal{N}$ is maximum-sized if and only if $M$ is isomorphic to a member of $\mathscr{G}_{r(M)}$ when $r(M) \neq 3$, or a member of $\mathscr{G}_{3} \cup\{B R \backslash p\}$ when $r(M)=3$.

A geometric representation of $B R \backslash p$ is given in Figure 2.19.

We then look at two more subclasses of golden-mean matroids, and show that Conjecture 1.2.3 is true in each of them. A $\mathbb{G}$-matroid $M$ is goldenmean-graphic if there exists a $\mathbb{G}$-representation $A$ of $M$ such that $A$ has at most two non-zero entries in each column. Firstly, in Chapter 5, we prove the following theorem. 
Theorem 5.1.2. Let $\mathcal{T}$ be the class of golden-mean-graphic matroids. Then

$$
h_{\mathcal{T}}(r)=\left(\begin{array}{c}
r+3 \\
2
\end{array}\right)-5 \text {. }
$$

Furthermore, $M \in \mathcal{T}$ is maximum-sized if and only if $M$ is isomorphic to either $T_{r(M)}^{2}$ or $G_{r(M)}$.

We then prove the following theorem in Chapter 6.

Theorem 6.1.4. Let $\mathcal{M}$ be the set of golden-mean matroids that have a spanning clique. Let $\mathcal{C}$ be the family of minors of matroids in $\mathcal{M}$. Then

$$
h_{\mathcal{C}}(r)= \begin{cases}\left(\begin{array}{c}
r+3 \\
2
\end{array}\right)-5 & \text { if } r \neq 3 \\
11 & \text { if } r=3\end{cases}
$$

Furthermore, $M \in \mathcal{C}$ is maximum-sized if and only if $M$ is isomorphic to a member of $\mathscr{G}_{r(M)}$ when $r(M) \neq 3$, or $M$ is isomorphic to the Betsy Ross when $r(M)=3$.

We anticipate that Theorem 6.1.4 will lead to a proof of Conjecture 1.2.3 for golden-mean matroids of sufficiently large rank.

Unless specified otherwise, all work in this thesis is original. Various results in Chapters 3 and 4 are similar to results from Welsh [21] due to the similar nature of the problems covered.

We now define some terminology that will be used throughout this thesis.

Definition 1.2.4. A point of a matroid is a rank-one flat. A line of a matroid is a rank-two flat. A long line of a matroid is a rank-two flat that contains at least three distinct rank-one flats. A very long line of a matroid is a rank-two flat that contains at least four distinct rank-one flats. The length of a line is the number of distinct rank-one flats contained in the line.

All undefined concepts and notation can be found in Matroid Theory by Oxley [10]. We also refer the reader to that book for an introduction to matroid theory. 
Projective geometry is all geometry.

Arthur Cayley

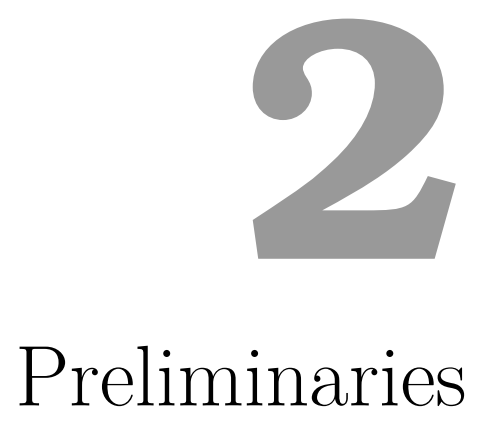

\section{$2.1 \quad$ Sage}

In this thesis, we prove various results using computers. The software we use is Sage [19], in particular the matroids package [14]. All code is available at http://michael.welsh.co.nz/thesis_code.

\subsubsection{Partial Fields}

In order to use Sage most conveniently, we need to find a field of prime order that captures $\mathbb{G}$. To do this, we use Pendavingh and Van Zwam's Lift Theorem. Before stating the theorem, we give some definitions related to partial fields. All these definitions can be found in Van Zwam's PhD thesis [20].

Definition 2.1.1. If $\mathfrak{M}$ is a $\mathbb{P}$-matrix with columns labelled by elements of $X$ and rows labelled by elements of $Y$, then we say that $\mathfrak{M}$ is a $X \times Y$ $\mathbb{P}$-matrix. 
Definition 2.1.2. Let $\mathfrak{M}$ be an $X \times Y \mathbb{P}$-matrix, and let $x \in X, y \in Y$ be such that $\mathfrak{M}_{x y} \in \mathbb{P}^{*}$. Then we define $\mathfrak{M}^{x y}$ to be the $(X-x) \cup y \times(Y-y) \cup x$ matrix with entries

$$
\left(\mathfrak{M}^{x y}\right)_{i j}= \begin{cases}\left(\mathfrak{M}_{x y}\right)^{-1} & \text { if } i j=y x \\ \left(\mathfrak{M}_{x y}\right)^{-1} \mathfrak{M}_{x j} & \text { if } i=y, j \neq x \\ -\mathfrak{M}_{i y}\left(\mathfrak{M}_{x y}\right)^{-1} & \text { if } j=x, i \neq y \\ \mathfrak{M}_{i j}-\mathfrak{M}_{i y}\left(\mathfrak{M}_{x y}\right)^{-1} \mathfrak{M}_{x j} & \text { otherwise }\end{cases}
$$

We say that $\mathfrak{M}^{x y}$ is obtained from $\mathfrak{M}$ by pivoting over $x y$.

Definition 2.1.3. Let $\mathfrak{M}$ and $\mathfrak{M}^{\prime}$ be $X \times Y \mathbb{P}$-matrices. We say that $\mathfrak{M}$ and $\mathfrak{M}^{\prime}$ are scaling-equivalent, denoted by $\mathfrak{M} \sim \mathfrak{M}^{\prime}$, if $\mathfrak{M}^{\prime}$ can be obtained from $\mathfrak{M}$ by scaling rows and columns by elements from $\mathbb{P}^{*}$.

Definition 2.1.4. Let $\mathfrak{M}$ be an $X \times Y \mathbb{P}$-matrix. We say that $\mathfrak{N}$ is a minor of $\mathfrak{M}$ if $\mathfrak{N}$ can be obtained from $\mathfrak{M}$ by a sequence of the following operations:

(i) Permuting rows or columns (and permuting labels accordingly);

(ii) Multiplying the entries of a row or column by an element of $\mathbb{P}^{*}$;

(iii) Deleting rows or columns;

(iv) Pivoting over a nonzero entry.

Definition 2.1.5. Let $\mathfrak{M}$ be an $X \times Y \mathbb{P}$-matrix, and let $\mathfrak{M}^{\prime}$ be an $X^{\prime} \times Y^{\prime}$ $\mathbb{P}$-matrix. Then $\mathfrak{M}$ and $\mathfrak{M}^{\prime}$ are isomorphic if there exist bijections $f: X \rightarrow$ $X^{\prime}$ and $g: Y \rightarrow Y^{\prime}$, such that for all $x \in X$ and $y \in Y, \mathfrak{M}_{x y}=\mathfrak{M}_{f(x) g(y)}^{\prime}$.

Definition 2.1.6. Let $\mathfrak{N}$ be isomorphic to a minor of the $\mathbb{P}$-matrix $\mathfrak{M}$. We denote this by $\mathfrak{N} \preceq \mathfrak{M}$.

Definition 2.1.7. Let $\mathbb{P}_{1}$ and $\mathbb{P}_{2}$ be partial fields. A function $\varphi: \mathbb{P}_{1} \rightarrow \mathbb{P}_{2}$ is a partial-field homomorphism if

(i) $\varphi(1)=1$;

(ii) For all $p, q \in \mathbb{P}_{1}, \varphi(p q)=\varphi(p) \varphi(q)$;

(iii) For all $p, q, r \in \mathbb{P}_{1}$ such that $p+q=r, \varphi(p)+\varphi(q)=\varphi(r)$.

Definition 2.1.8. Let $\mathbb{P}$ be a partial field. An element $p \in \mathbb{P}$ is fundamental if $1-p \in \mathbb{P}$. We denote the set of fundamental elements of a partial field by $\mathscr{F}(\mathbb{P})$. 
Definition 2.1.9. Let $\mathfrak{M}$ be a $\mathbb{P}$-matrix. We define the cross ratios of $\mathfrak{M}$ as the set

$$
\operatorname{Cr}(\mathfrak{M})=\left\{p \mid\left[\begin{array}{ll}
1 & 1 \\
p & 1
\end{array}\right] \preceq \mathfrak{M}\right\}
$$

\subsubsection{The Lift Theorem}

We can now state the Lift Theorem.

Theorem 2.1.10 (Lift Theorem [13, Theorem 3.5]). Let $\mathbb{P}$ and $\widehat{\mathbb{P}}$ be two partial fields, let $\mathfrak{M}$ be a $\mathbb{P}$-matrix, and let $\varphi: \widehat{\mathbb{P}} \rightarrow \mathbb{P}$ be a homomorphism such that the restriction of $\varphi$ to the fundamental elements, $\left.\varphi\right|_{\mathscr{F}(\widehat{\mathbb{P}})}: \mathscr{F}(\widehat{\mathbb{P}}) \rightarrow$ $\mathscr{F}(\mathbb{P})$, is a bijection. Then exactly one of the following is true:

(i) There is a $\widehat{\mathbb{P}}$-matrix $\widehat{\mathfrak{M}}$ such that $\varphi(\widehat{\mathfrak{M}}) \sim \mathfrak{M}$.

(ii) $\mathfrak{M}$ has a minor $\mathfrak{N}$ such that

a) There is no $\widehat{\mathbb{P}}$-matrix $\widehat{\mathfrak{N}}$ such that $\varphi(\widehat{\mathfrak{N}})=\mathfrak{N}$;

b) $\mathfrak{N}$ or $\mathfrak{N}^{T}$ equals

$$
\left[\begin{array}{llll}
0 & 1 & 1 & 1 \\
1 & 0 & 1 & 1 \\
1 & 1 & 0 & 1
\end{array}\right] \text { or }\left[\begin{array}{lll}
1 & 1 & 1 \\
1 & p & q
\end{array}\right]
$$

for some distinct $p, q \in \mathscr{F}(\mathbb{P})-\{0,1\}$.

The following result, that captures $\mathbb{G}$ inside of $G F(19)$, is due to Pendavingh (personal communication).

Lemma 2.1.11. Let $M$ be a golden-mean matroid. Then $M$ can be represented by a matrix $\mathfrak{M}$ over $G F(19)$ such that all cross ratios of $\mathfrak{M}$ are in $S=\{0,1,4,5,6,14,15,16\}$. Furthermore, all matrices of this type represent golden-mean matroids.

Proof. There is a unique partial field homomorphism from $\mathbb{G}$ to $G F(19)$ that sends $\tau$ to 5 . Let $\psi$ be this homomorphism. The fundamental elements of $\mathbb{G}$ are $0,1, \tau,-\tau, \tau^{-1},-\tau^{-1}, \tau^{2}$, and $\tau^{-2}([20$, Lemma 2.5.19]). Calculation shows that $\psi$ operates on $\mathscr{F}(\mathbb{G})$ as shown in Table 2.1.

Hence $\psi$ satisfies the conditions of Theorem 2.1.10 with $\widehat{\mathbb{P}}$ equal to $\mathbb{G}$ and $\mathbb{P}$ equal to $G F(19)$. 


\begin{tabular}{|c|c|c|c|c|c|c|c|}
\hline$x$ & $\psi(x)$ & $x$ & $\psi(x)$ & $x$ & $\psi(x)$ & $x$ & $\psi(x)$ \\
\hline 0 & 0 & $\tau$ & 5 & $\tau^{-1}$ & 4 & $\tau^{2}$ & 6 \\
\hline & 1 & $-\tau$ & 14 & $-\tau^{-1}$ & 15 & $\tau^{-2}$ & 16 \\
\hline
\end{tabular}

Table 2.1: Values of $\psi$, the unique homomorphism from $\mathbb{G}$ to $G F(19)$ such that $\psi(\tau)=5$

Let $\mathfrak{M}$ be a $\mathbb{G}$-matrix that represents the $\mathbb{G}$-matroid $M$. Then $\psi(\mathfrak{M})$ represents $M$ over $G F(19)$. Since every cross-ratio of $\mathfrak{M}$ is a fundamental element of $\mathbb{G}$, it follows that every cross-ratio of $\psi(\mathfrak{M})$ is the image of a fundamental element under the homomorphism $\psi$, so one direction of the lemma is proved. For the converse, we let $\mathfrak{M}$ be a $G F(19)$ matrix whose cross-ratios are all in $S$, and let $M$ be the matroid represented by $\mathfrak{M}$. If condition (i) holds in $\mathfrak{M}$, then $M$ can be represented by a $\mathbb{G}$-matrix, so there is nothing left to prove. Therefore we assume that condition (ii) holds. The Sage code Matroid(reduced_matrix $=\operatorname{matrix}(\operatorname{GF}(19),[[0,1,1,1]$, $[1,0,1,1],[1,1,0,1]]))$. cross_ratios() tells us that the crossratios of the Fano-type matrix are $\{2,8,10\}$, so $\mathfrak{M}$ has a minor equal to

$$
\mathfrak{N}=\left[\begin{array}{lll}
1 & 1 & 1 \\
1 & p & q
\end{array}\right]
$$

for some distinct $p$ and $q$. Since $p$ and $q$ are both cross-ratios of $\mathfrak{N}$, it follows that $\{p, q\} \subset\{4,5,6,14,15,16\}$. We consider all possible values of $p$ and $q$ such that the cross-ratios of $\mathfrak{N}$ are in $S$, and we show that in each case, if we replace $p$ and $q$ with their pre-images from Table 2.1, then we obtain a $\mathbb{G}$-matrix. To do this, we compute $z=x-y$ for $\{x, y\} \subset$ $\left\{\tau,-\tau, \tau^{-1},-\tau^{-1}, \tau^{2}, \tau^{-2}\right\}$ and check if $z$ is in $\left\{ \pm \tau^{i} \mid i \in \mathbb{Z}\right\}$. We do this using the Sage code in Appendix 2.A.3. This shows that condition (ii) in Theorem 2.1.10 does not hold.

Using Lemma 2.1.11, we are able to check if a given $G F(19)$ matrix represents a golden-mean matroid or not. To do this, we use the function is_gm( ), detailed in Appendix 2.A.2. 


\subsection{3 $G F(4)$}

Throughout this work, $G F(4)$ will consist of $\left\{0,1, \alpha, \alpha^{2}\right\}$, where $\alpha^{2}=\alpha+1$. A common problem in representable matroid research is that of inequivalent representations. However, matroids are uniquely representable over $G F(4)$.

Lemma 2.1.12 (Kahn [7, Theorem 1]). If $M$ is 3-connected and representable over $G F(4)$ then it is uniquely representable over $G F(4)$.

Because of Lemma 2.1.12, we would like to do all matrix work over $G F(4)$. As such, we use the following homomorphism from $\mathbb{G}$ to $G F(4)$ to create $G F(4)$-matrices from $\mathbb{G}$-matrices.

$$
\begin{gathered}
f: 0 \longmapsto 0 \\
f: 1 \longmapsto 1 \\
f: \tau^{k} \longmapsto \alpha^{k} .
\end{gathered}
$$

Verifying that this is a homomorphism is an easy exercise.

\subsection{The Three Families}

In this thesis, there are three families of interest. In this section, we introduce the families and give various representations of them.

The three families are the $T_{r}^{2}, G_{r}$, and $H P_{r}$ families. The $T_{r}^{2}$ family was introduced by Semple [16], and the $G_{r}$ and $H P_{r}$ families were introduced by Archer [1]. All three families were shown to be golden-mean by Archer [1, Proposition 5.3].

To make the notation easier, we use $\mathscr{G}_{r}$ to refer to the set $\left\{T_{r}^{2}, G_{r}, H P_{r}\right\}$.

\subsection{1 $\mathbb{G}$-matrices}

We introduce the three families as $\mathbb{G}$-matrices. These are shown in Figure 2.1. The block $D_{k}$ represents the $k \times\left(\begin{array}{l}k \\ 2\end{array}\right)$ matrix whose columns consist of all $k$ - 
tuples with two non-zero entries, with the first being 1 and the second being -1 . The block $0_{m}^{n}$ denotes the $n \times m$ matrix consisting entirely of zeros.

The first family is the $T_{r}^{2}$ family. Note that the matroid $T_{r}^{2}$ contains $r-1$ copunctual five-point lines. A geometric representation of $T_{3}^{2}$ is given in Figure 2.2.

The second family is the $G_{r}$ family. Note that the matroid $G_{r}$ has one fivepoint line and $2(r-2)$ four-point lines, all of which meet the five-point line. A geometric representation of $G_{3}$ is given in Figure 2.3.

The final family is the $H P_{r}$ family. Note that the matroid $H P_{r}$ has one five-point line and no four-point lines. A geometric representation of $\mathrm{HP}_{3}$ is given in Figure 2.4.

\subsection{2 $G F(4)$ Matrices}

We apply the homomorphism $f$ from Section 2.1.3 to the $\mathbb{G}$-matrix of each of the three families. This yields the $G F(4)$ matrices in Figure 2.5.

\subsubsection{Minors}

We prove the following lemma.

Lemma 2.2.1. Let $M$ be a member of $\mathscr{G}_{r}$. Let $M^{\prime}$ be isomorphic to $\operatorname{si}(M / e)$ for some e $\in E(M)$.

(i) If $M$ is isomorphic to $T_{r}^{2}$, then $M^{\prime}$ is isomorphic to $T_{r-1}^{2}$ or $M\left(K_{r}\right)$.

(ii) If $M$ is isomorphic to $G_{r}$, then $M^{\prime}$ is isomorphic to $G_{r-1}, T_{r-1}^{2}$, or $T_{r-1}^{1}$.

(iii) If $M$ is isomorphic to $H P_{r}$, then $M^{\prime}$ is isomorphic to $H P_{r-1}, T_{r-1}^{2}$, or $T_{r-1}^{1}$.

Proof. We prove this by considering the $G F(4)$ matrices from Figure 2.5 and pivoting on all possible entries. We do this in three near-identical sublemmas. 


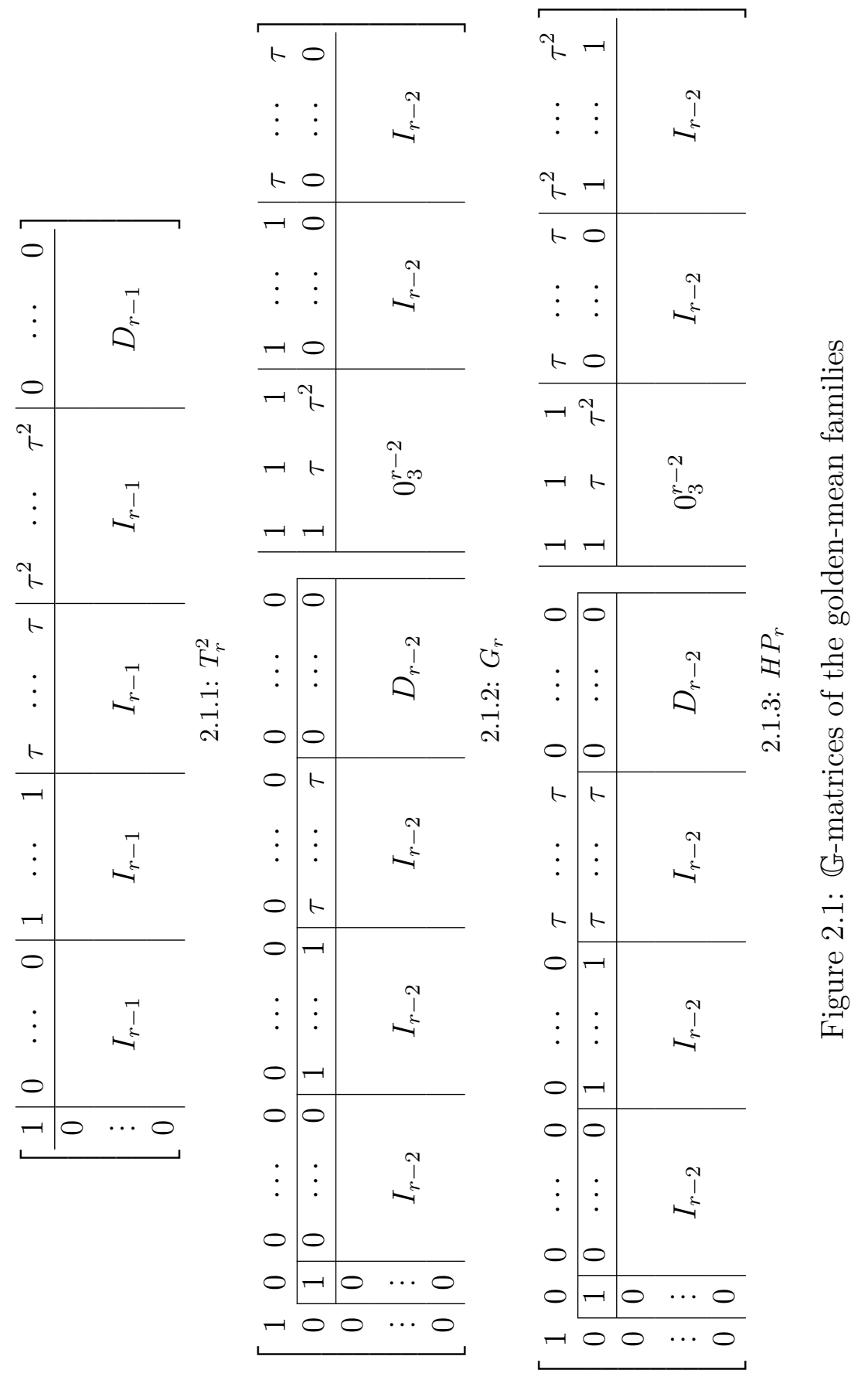




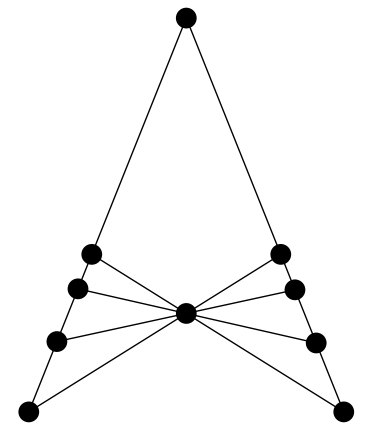

Figure 2.2: $T_{3}^{2}$

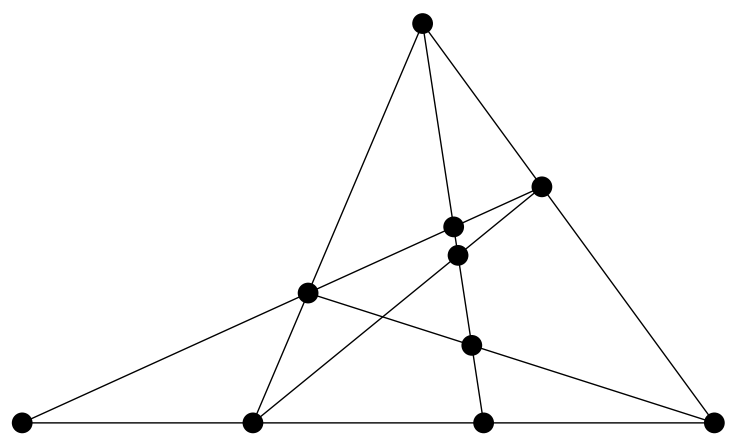

Figure 2.3: $G_{3}$

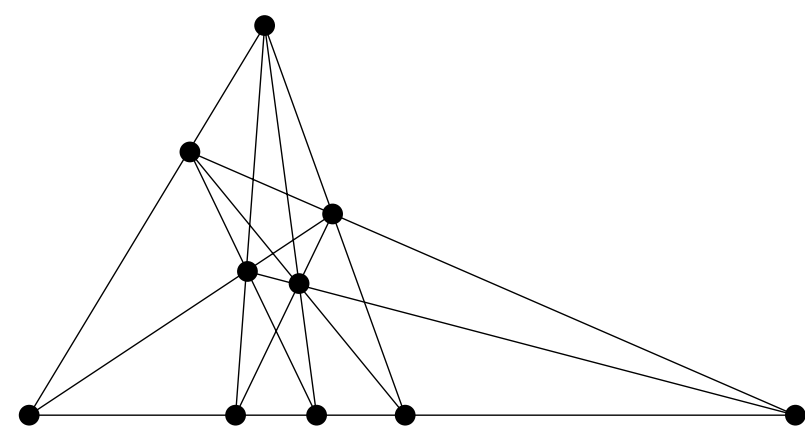

Figure 2.4: $\mathrm{HP}_{3}$ 


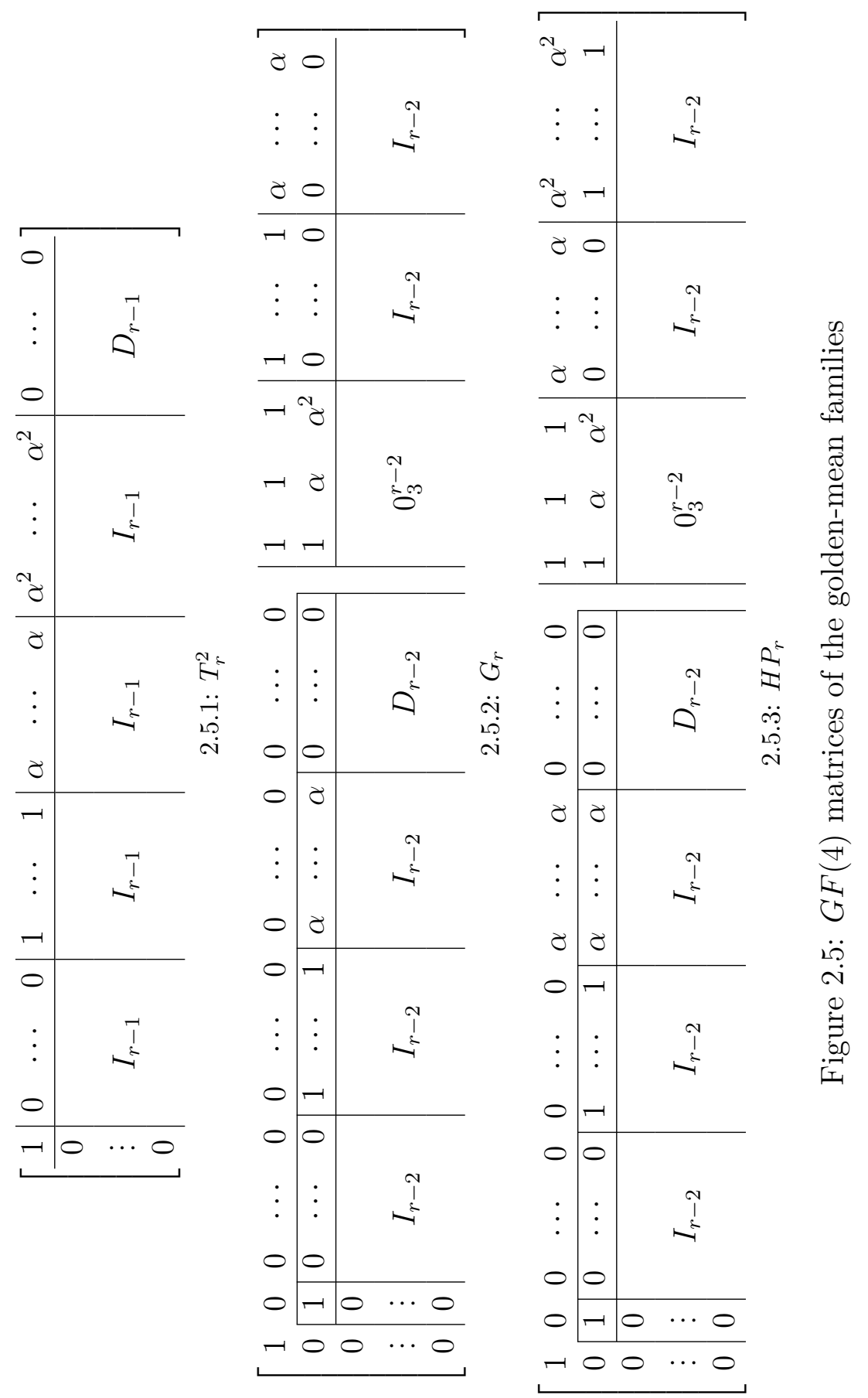


Throughout this proof, let

$$
I_{k}^{\prime}=\left[\begin{array}{ccc|c} 
& & 0 \\
& I_{k-1} & & \vdots \\
& & & 1 \\
& & & 1
\end{array}\right] .
$$

Sublemma 2.2.1.1. If $M$ is isomorphic to $T_{r}^{2}$, then $M^{\prime}$ is isomorphic to $T_{r-1}^{2}$ or $M\left(K_{r}\right)$.

Subproof. Consider the $G F(4)$ matrix $\mathfrak{M}$ for $T_{r}^{2}$ in Figure 2.5.1. Let $A$ be the set consisting of the first column of $\mathfrak{M}$, and let $B, C, D, E$ and $F$ be the subsequent blocks of columns of $\mathfrak{M}$. Note that $|A|=1,|B|=|C|=|D|=$ $|E|=r-1$, and $|F|=\left(\begin{array}{c}r-1 \\ 2\end{array}\right)$.

We now contract an element from each set. It is relatively easy to see that if $X$ is a block, and $x, y \in X$ are elements of $M$, then $\operatorname{si}(M / x) \cong \operatorname{si}(M / y)$.

Let $e$ be an element of $E(M)$.

$\boldsymbol{e} \in \boldsymbol{A}$ In this case, we can see that $\operatorname{si}(M / e) \cong M\left(K_{r}\right)$.

$\boldsymbol{e} \in \boldsymbol{B}$ Let $e$ be the last element of $B$. We can see that $\operatorname{si}(M / e) \cong T_{r-1}^{2}$.

$\boldsymbol{e} \in \boldsymbol{C}$ Let $e$ be the last element of $C$. We pivot by adding the last row to the first row, getting the matrix shown in Figure 2.6.1. Now, when we delete the last row and suppress parallel elements, we can see that $\operatorname{si}(M / e) \cong T_{r-1}^{2}$.

$\boldsymbol{e} \in \boldsymbol{D}$ Let $e$ be the last element of $D$. We pivot by adding the last row scaled by $\alpha$ to the first row, getting the matrix shown in Figure 2.6.2. Now, when we delete the last row and suppress parallel elements, we can see that $\operatorname{si}(M / e) \cong T_{r-1}^{2}$.

$\boldsymbol{e} \in \boldsymbol{E}$ Let $e$ be the last element of $E$. We pivot by adding the last row scaled by $\alpha^{2}$ to the first row, getting the matrix shown in Figure 2.6.3. Now, 


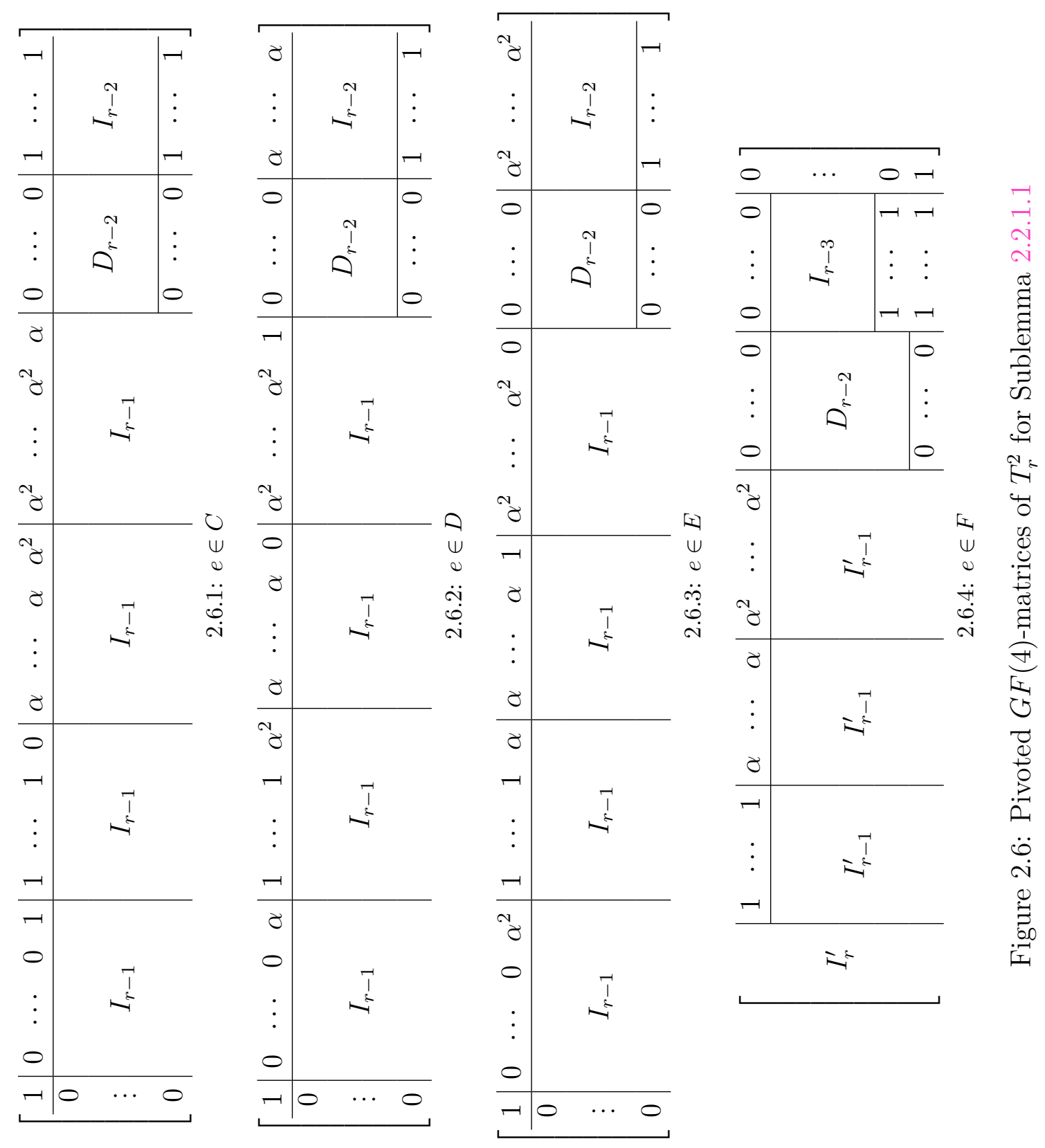


when we delete the last row and suppress parallel elements, we can see that $\operatorname{si}(M / e) \cong T_{r-1}^{2}$.

$\boldsymbol{e} \in \boldsymbol{F}$ Let $e$ be the last element of $F$. We pivot by adding the last row to the second-last row, getting the matrix shown in Figure 2.6.4. Now, when we delete the last row and suppress parallel elements, we can see that $\operatorname{si}(M / e) \cong$ $T_{r-1}^{2}$.

Sublemma 2.2.1.2. If $M$ is isomorphic to $G_{r}$, then $M^{\prime}$ is isomorphic to $G_{r-1}, T_{r-1}^{2}$, or $T_{r-1}^{1}$.

Subproof. Consider the $G F(4)$ matrix $\mathfrak{M}$ for $G_{r}$ in Figure 2.5.2. Let $A$ be the set consisting of the first column of $\mathfrak{M}$. Let $B$ be the set consisting of the second column of $\mathfrak{M}$. Let $C, D, E$, and $F$ be the next four blocks of columns of $\mathfrak{M}$. For each of the next three columns, let the set consisting of this column of $\mathfrak{M}$ be $G, H$, and $I$, respectively. Let $J$ and $K$ be the last two blocks of columns of $\mathfrak{M}$. Note that $|A|=|B|=|G|=|H|=|I|=1$, $|C|=|D|=|E|=|J|=|K|=r-2$, and $|F|=\left(\begin{array}{c}r-2 \\ 2\end{array}\right)$.

We now contract an element from each set. It is relatively easy to see that if $X$ is a block, and $x, y \in X$ are elements of $M$, then $\operatorname{si}(M / x) \cong \operatorname{si}(M / y)$.

Let $e$ be an element of $E(M)$.

$\boldsymbol{e} \in \boldsymbol{A}$ In this case, we can see that $\operatorname{si}(M / e) \cong T_{r-1}^{1}$.

$\boldsymbol{e} \in \boldsymbol{B}$ In this case, we can see that $\operatorname{si}(M / e) \cong T_{r-1}^{1}$.

$\boldsymbol{e} \in \boldsymbol{C}$ Let $e$ be the last element of $C$. We can see that $\operatorname{si}(M / e) \cong G_{r-1}$.

$\boldsymbol{e} \in \boldsymbol{D}$ Let $e$ be the last element of $D$. We pivot by adding the last row to the second row, getting the matrix shown in Figure 2.7.1. Now, when we delete the last row and suppress parallel elements, we can see that $\operatorname{si}(M / e) \cong$ $G_{r-1}$. 


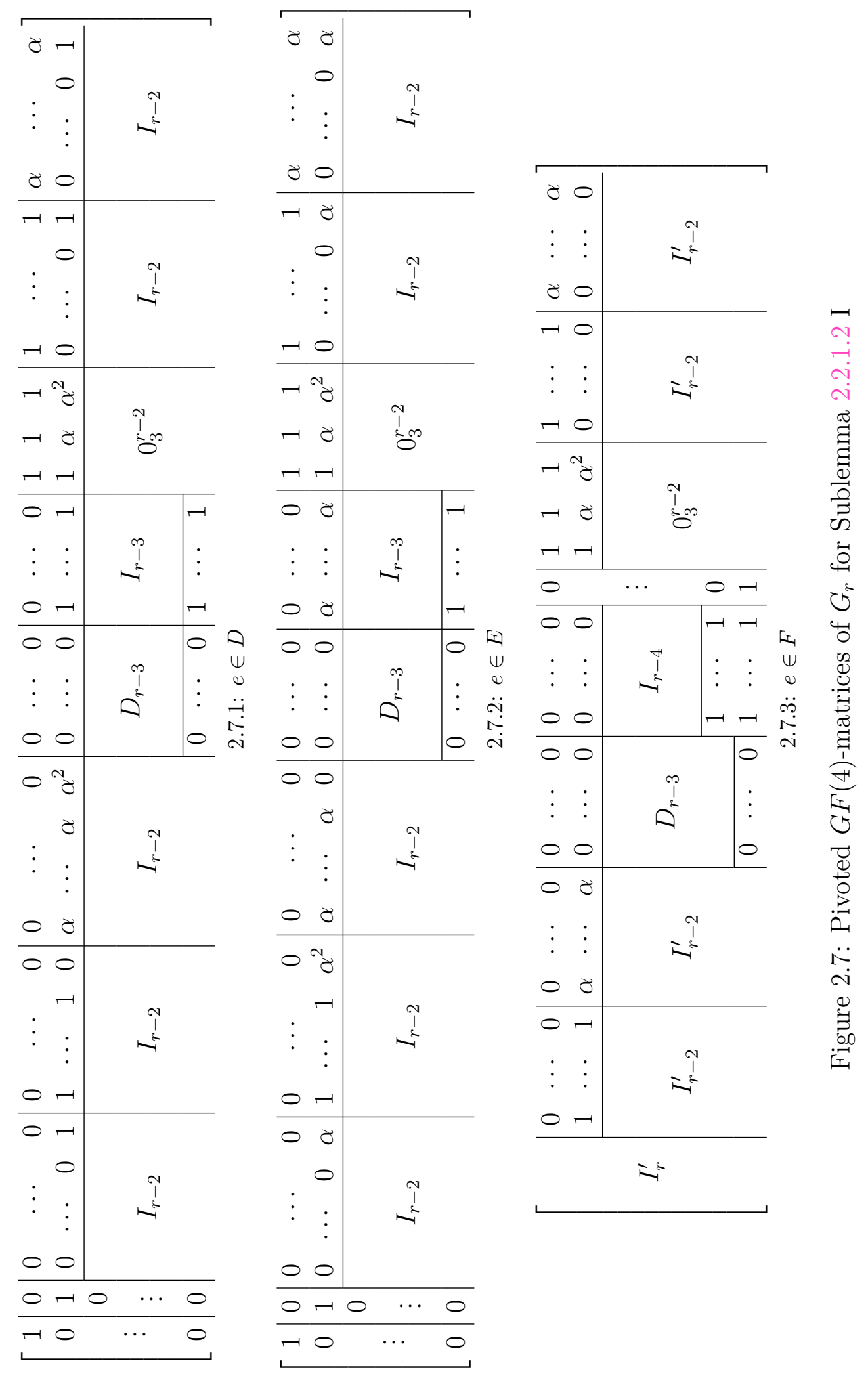


$\boldsymbol{e} \in \boldsymbol{E}$ Let $e$ be the last element of $E$. We pivot by adding the last row scaled by $\alpha$ to the second row, getting the matrix shown in Figure 2.7.2. Now, when we delete the last row and suppress parallel elements, we can see that $\operatorname{si}(M / e) \cong G_{r-1}$.

$\boldsymbol{e} \in \boldsymbol{F} \quad$ Let $e$ be the last element of $F$. We pivot by adding the last row to the second-last row, getting the matrix shown in Figure 2.7.3. Now, when we delete the last row and suppress parallel elements, we can see that $\operatorname{si}(M / e) \cong$ $G_{r-1}$.

$\boldsymbol{e} \in \boldsymbol{G}$ We pivot by adding the first row to the second row, getting the matrix shown in Figure 2.8.1. Now, when we delete the first row and suppress parallel elements, we can see that $\operatorname{si}(M / e) \cong T_{r-1}^{1}$.

$\boldsymbol{e} \in \boldsymbol{H}$ We pivot by adding the first row scaled by $\alpha$ to the second row, getting the matrix shown in Figure 2.8.2. Now, when we delete the first row and suppress parallel elements, we can see that $\operatorname{si}(M / e) \cong T_{r-1}^{2}$.

$\boldsymbol{e} \in \boldsymbol{I}$ We pivot by adding the first row scaled by $\alpha^{2}$ to the second row, getting the matrix shown in Figure 2.8.3. Now, when we delete the first row and suppress parallel elements, we can see that $\operatorname{si}(M / e) \cong T_{r-1}^{2}$.

$\boldsymbol{e} \in \boldsymbol{J}$ Let $e$ be the last element of $J$. We pivot by adding the last row to the first row, getting the matrix shown in Figure 2.9.1. Now, when we delete the last row and suppress parallel elements, we can see that $\operatorname{si}(M / e) \cong G_{r-1}$.

$\boldsymbol{e} \in \boldsymbol{K}$ Let $e$ be the last element of $K$. We pivot by adding the last row scaled by $\alpha$ to the first row, getting the matrix shown in Figure 2.9.2. Now, when we delete the last row and suppress parallel elements, we can see that $\operatorname{si}(M / e) \cong G_{r-1}$.

Sublemma 2.2.1.3. If $M$ is isomorphic to $H P_{r}$, then $M^{\prime}$ is isomorphic to $H P_{r-1}, T_{r-1}^{2}$, or $T_{r-1}^{1}$. 


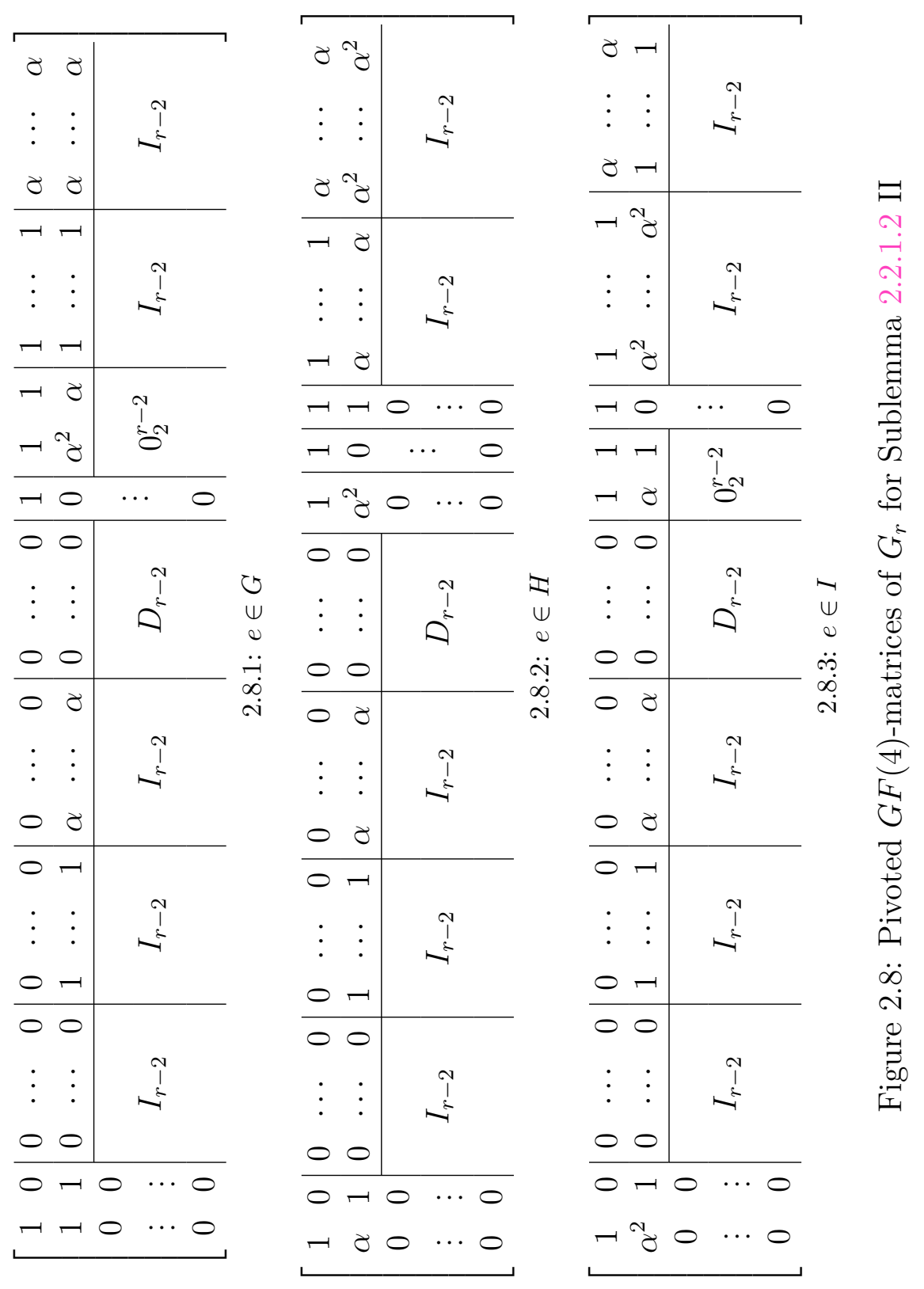




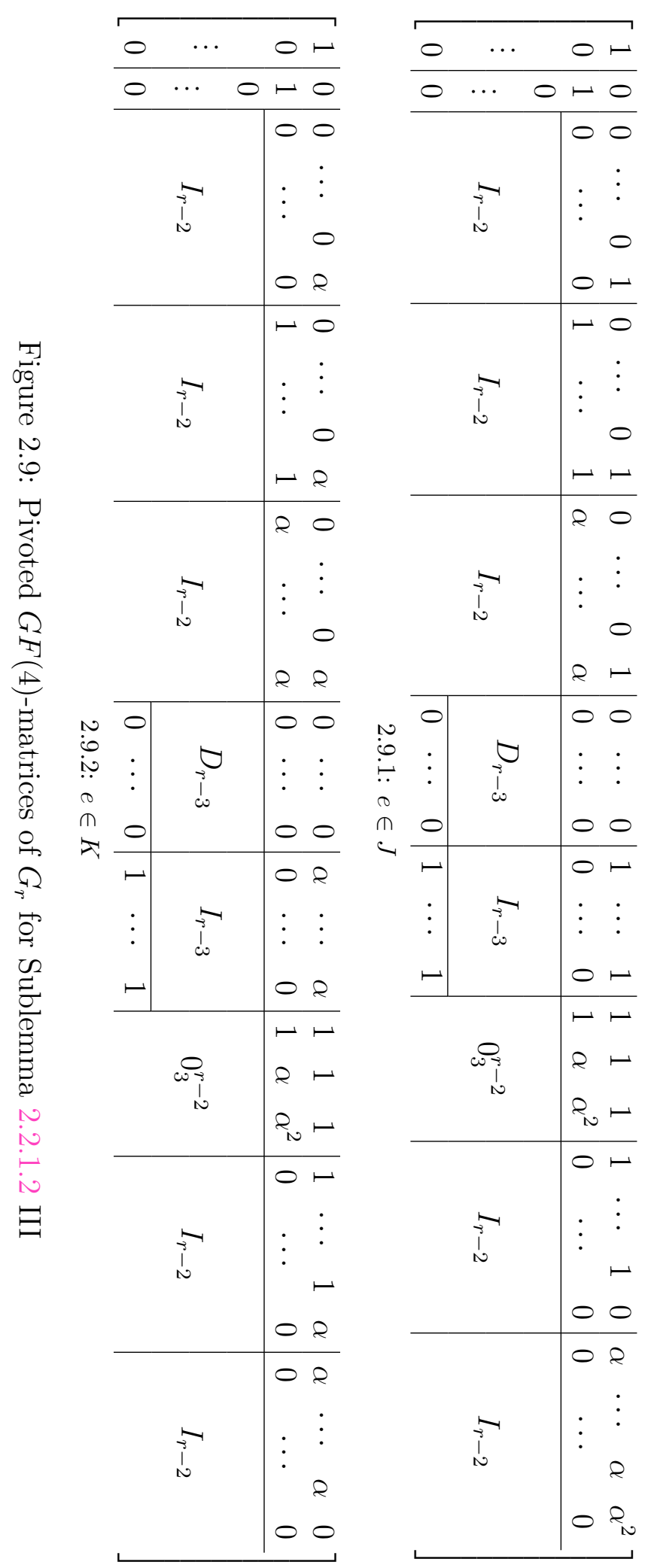


Subproof. Consider the $G F(4)$ matrix $\mathfrak{M}$ for $H P_{r}$ in Figure 2.5.3. Let $A$ be the set consisting of the first column of $\mathfrak{M}$. Let $B$ be the set consisting of the second column of $\mathfrak{M}$. Let $C, D, E$, and $F$ be the next four blocks of columns of $\mathfrak{M}$. For each of the next three columns, let the set consisting of this column of $\mathfrak{M}$ be $G, H$, and $I$, respectively. Let $J$ and $K$ be the last two blocks of columns of $\mathfrak{M}$. Note that $|A|=|B|=|G|=|H|=|I|=1$, $|C|=|D|=|E|=|J|=|K|=r-2$, and $|F|=\left(\begin{array}{c}r-2 \\ 2\end{array}\right)$.

We now contract an element from each set. It is relatively easy to see that if $X$ is a block, and $x, y \in X$ are elements of $M$, then $\operatorname{si}(M / x) \cong \operatorname{si}(M / y)$.

Let $e$ be an element of $E(M)$.

$\boldsymbol{e} \in \boldsymbol{A}$ In this case, we can see that $\operatorname{si}(M / e) \cong T_{r-1}^{1}$

$\boldsymbol{e} \in \boldsymbol{B}$ In this case, we can see that $\operatorname{si}(M / e) \cong T_{r-1}^{2}$.

$\boldsymbol{e} \in \boldsymbol{C}$ Let $e$ be the last element of $C$. We can see that $\operatorname{si}(M / e) \cong H P_{r-1}$.

$\boldsymbol{e} \in \boldsymbol{D}$ Let $e$ be the last element of $D$. We pivot by adding the last row to the second row, getting the matrix shown in Figure 2.10.1. Now, when we delete the last row and suppress parallel elements, we can see that $\operatorname{si}(M / e) \cong$ $H P_{r-1}$.

$\boldsymbol{e} \in \boldsymbol{E}$ Let $e$ be the last element of $E$. We pivot by adding the last row scaled by $\alpha$ to the first and second rows, getting the matrix shown in Figure 2.10.2. Now, when we delete the last row and suppress parallel elements, we can see that $\operatorname{si}(M / e) \cong H P_{r-1}$.

$\boldsymbol{e} \in \boldsymbol{F}$ Let $e$ be the last element of $F$. We pivot by adding the last row to the second-last row, getting the matrix shown in Figure 2.10.3. Now, when we delete the last row and suppress parallel elements, we can see that $\operatorname{si}(M / e) \cong H P_{r-1}$.

$\boldsymbol{e} \in \boldsymbol{G}$ We pivot by adding the first row to the second row, getting the matrix shown in Figure 2.11.1. Now, when we delete the first row and suppress 


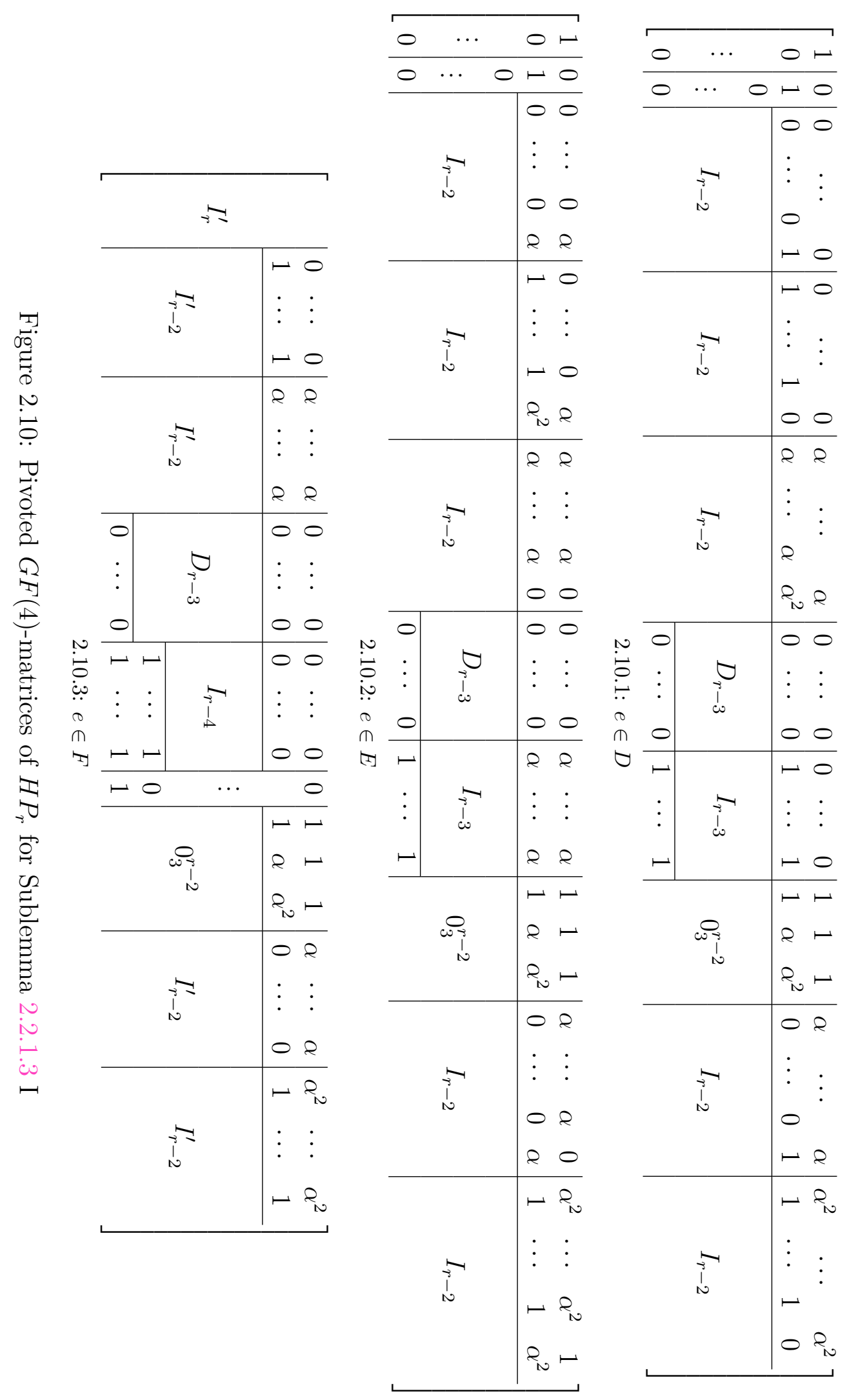



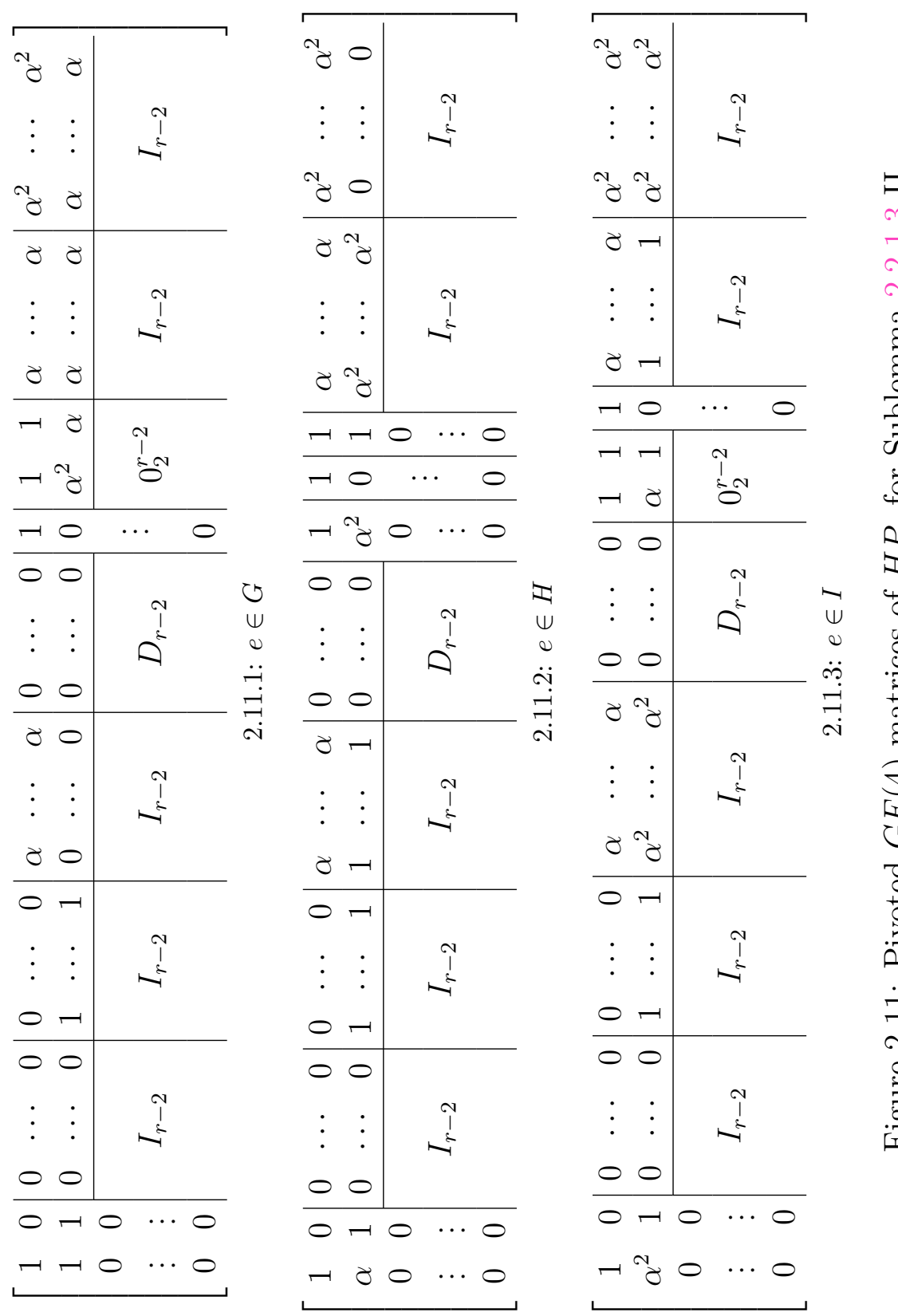

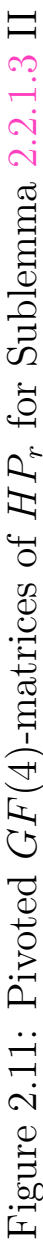
\begin{tabular}{llll}
0 & 0 & & \\
\hline 0 & -1 & $\cdots$ & 0
\end{tabular} $\neg{ }^{N} \circ \cdots$ 
parallel elements, we can see that $\operatorname{si}(M / e) \cong T_{r-1}^{1}$.

$\boldsymbol{e} \in \boldsymbol{H}$ We pivot by adding the first row scaled by $\alpha$ to the second row, getting the matrix shown in Figure 2.11.2. Now, when we delete the first row and suppress parallel elements, we can see that $\operatorname{si}(M / e) \cong T_{r-1}^{1}$.

$\boldsymbol{e} \in \boldsymbol{I}$ We pivot by adding the first row scaled by $\alpha^{2}$ to the second row, getting the matrix shown in Figure 2.11.3. Now, when we delete the first row and suppress parallel elements, we can see that $\operatorname{si}(M / e) \cong T_{r-1}^{1}$.

$\boldsymbol{e} \in \boldsymbol{J}$ Let $e$ be the last element of $J$. We pivot by adding the last row scaled by $\alpha$ to the first row, getting the matrix shown in Figure 2.12.1. Now, when we delete the last row and suppress parallel elements, we can see that $\operatorname{si}(M / e) \cong H P_{r-1}$.

$\boldsymbol{e} \in \boldsymbol{K}$ Let $e$ be the last element of $K$. We pivot by adding the last row scaled by $\alpha^{2}$ to the first row, and then adding the last row to the second row, getting the matrix shown in Figure 2.12.2. Now, when we delete the last row and suppress parallel elements, we can see that $\operatorname{si}(M / e) \cong H P_{r-1}$.

When we merge Sublemmas 2.2.1.1, 2.2.1.2, and 2.2.1.3, we get the desired result.

We are interested in the cases where $e \in E(M)$ is not in any five-point lines. The following corollary gives this characterisation.

Corollary 2.2.2. Let $M$ be a member of $\mathscr{G}_{r}$. Let $M^{\prime}$ be isomorphic to $\operatorname{si}(M / e)$ for some $e \in E(M)$ such that $e$ is not in any five-point lines.

(i) If $M$ is isomorphic to $T_{r}^{2}$, then $M^{\prime}$ is isomorphic to $T_{r-1}^{2}$.

(ii) If $M$ is isomorphic to $G_{r}$, then $M^{\prime}$ is isomorphic to $G_{r-1}$.

(iii) If $M$ is isomorphic to $H P_{r}$, then $M^{\prime}$ is isomorphic to $H P_{r-1}$.

\subsubsection{G-graphic representations}

Recall that a $\mathbb{G}$-matroid $M$ is $\mathbb{G}$-graphic if there exists a $\mathbb{G}$-representation $A$ of $M$ such that $A$ has at most two non-zero entries in each column. 


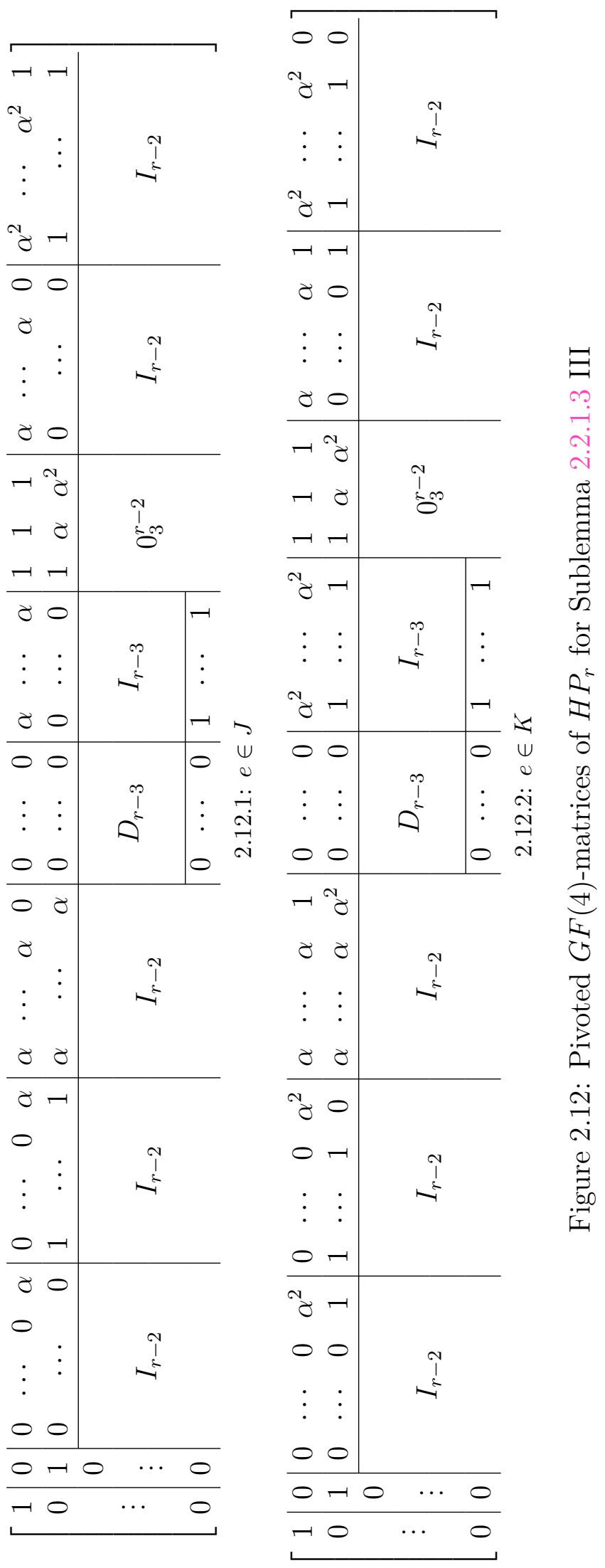




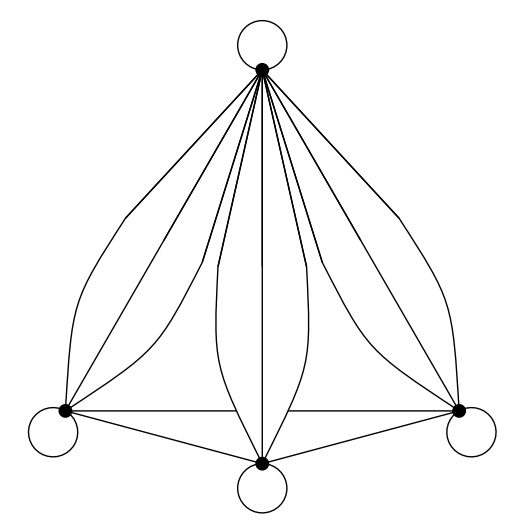

Figure 2.13: $\mathbb{G}$-graphic representation of $T_{4}^{2}$

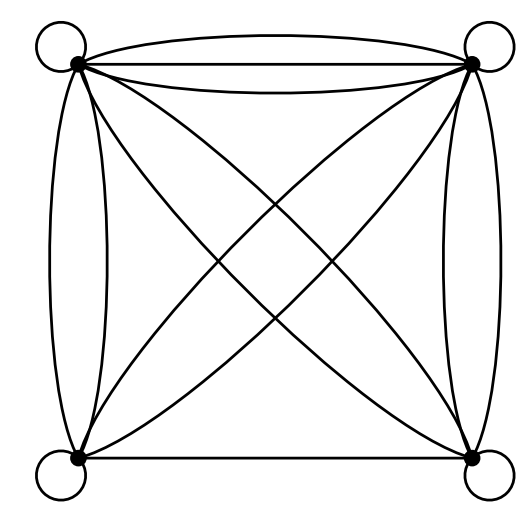

Figure 2.14: $\mathbb{G}$-graphic representation of $G_{4}$

Two of the families, $T_{r}^{2}$ and $G_{r}$ are $\mathbb{G}$-graphic matroids. The third family, $H P_{r}$, is not $\mathbb{G}$-graphic. We prove Conjecture 1.2.3 for the class of $\mathbb{G}$-graphic matroids in Chapter 5.

To construct a $\mathbb{G}$-graphic representation of $T_{r}^{2}$, take a collection of $r$ vertices, each with a loop. Select $r-1$ of these vertices and construct $K_{r-1}$. Finally, construct a parallel class of size three from the unselected vertex to each vertex in the $K_{r-1}$. A $\mathbb{G}$-graphic representation of $T_{4}^{2}$ is given in Figure 2.13. To construct a $\mathbb{G}$-graphic representation of $G_{r}$, take a collection of $r$ vertices, each with a loop. Select $r-2$ of these vertices and construct $K_{r-2}$. Between the two unselected vertices $u$ and $v$, construct a parallel class of size three. Finally, construct a parallel class of size two from each of $u$ and $v$ to each vertex in the $K_{r-2}$. A $\mathbb{G}$-graphic representation of $G_{4}$ is given in Figure 2.14. Note that $\mathbb{G}$-graphic representations are typically weighted and directed. However, we have left this information out. 


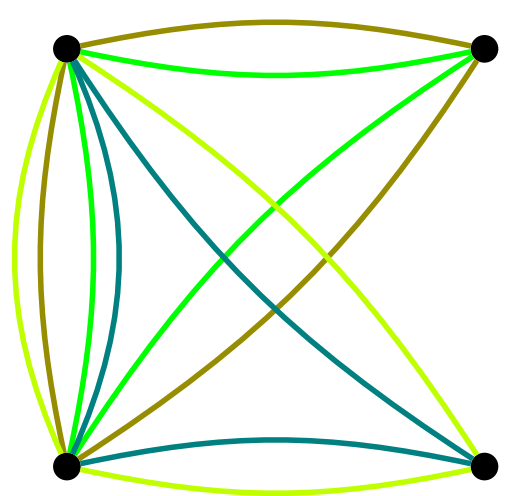

2.15.1: $T_{3}^{2}$

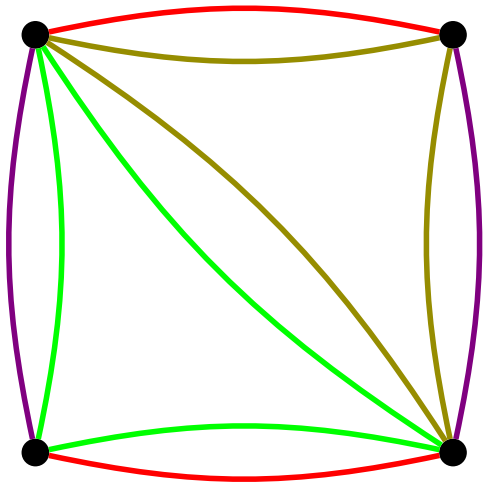

2.15.2: $G_{3}$

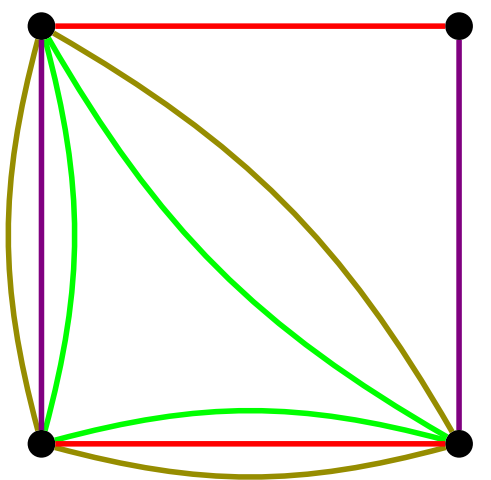

2.15.3: $\mathrm{HP}_{3}$

Figure 2.15: Augmented clique representations of all members of $\mathscr{G}_{3}$

\subsubsection{Augmented Cliques}

From looking at the matrices of the three families in Figure 2.1, it is easy to see that each of the families contains a spanning clique. In Chapter 6 we will show that there are two ways to extend from a spanning clique in a golden-mean way, called green triangles and red matchings. We collect this information in a graphical form, and construct augmented cliques. This construction is detailed in Section 6.2. Augmented clique representations of all members of $\mathscr{G}_{3}$ are given in Figure 2.15.

\section{$2.3 \quad$ Rank Three}

In various places, we will need to know all rank-three golden-mean matroids. To this end, we have the following lemma. Note that this lemma also confirms 


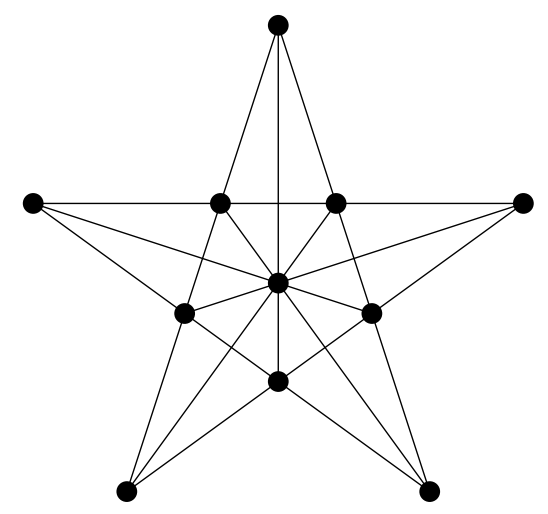

Figure 2.16: The Betsy Ross $(B R)$

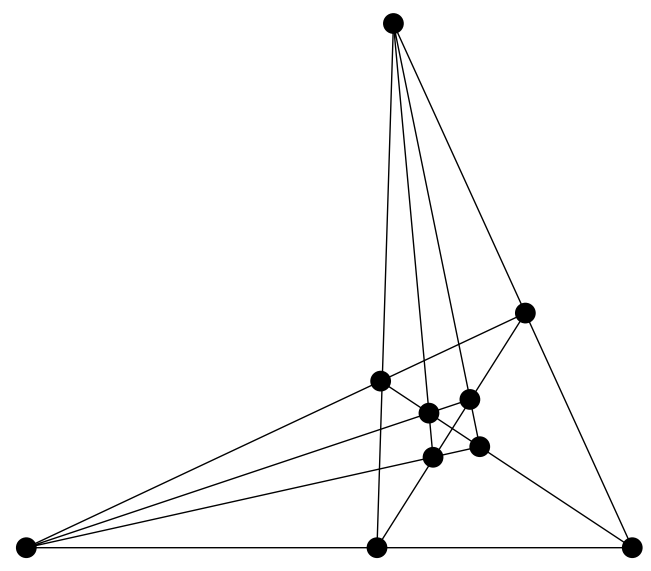

Figure 2.17: $Y_{10}$

Conjecture 1.2.3 for rank three.

Lemma 2.3.1. Let $M$ be a simple golden-mean matroid of rank three. Then $M$ is isomorphic to a restriction of one of the following matroids:

- BR, The Betsy Ross (Figure 2.16),

- $T_{3}^{2}$ (Figure 2.2),

- $G_{3}$ (Figure 2.3),

- $\mathrm{HP}_{3}$ (Figure 2.4), or

- $Y_{10}$ (Figure 2.17).

Proof. We prove this using a computer search, which is detailed in Appendix 2.A.4. This also follows from independent work by Archer [1], Hliněný (personal correspondence), and Pendavingh (personal correspondence).

There are two ten-element restrictions of $B R$. These are $S_{10}$ and $B R \backslash p$. A geometric representation of $S_{10}$ is given in Figure 2.18 and a geometric 


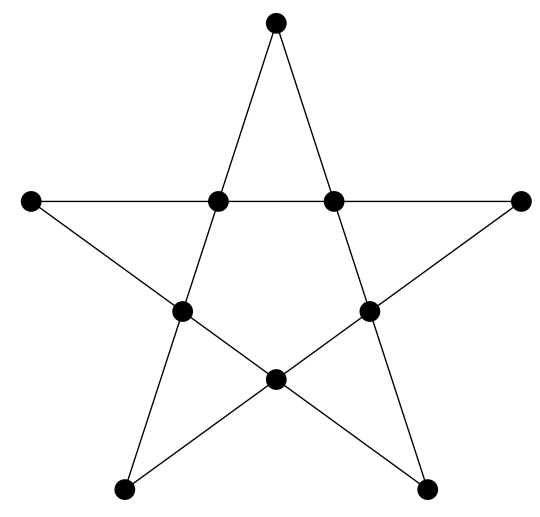

Figure 2.18: $S_{10}$

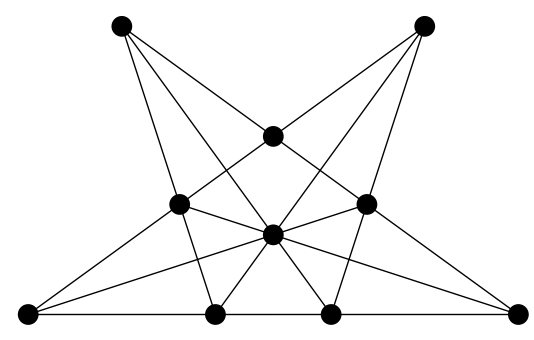

Figure 2.19: $B R \backslash p$

representation of $B R \backslash p$ is given in Figure 2.19.

\section{$2.4 \quad 2$-rounded Sets}

We shall say that a matroid $M$ uses an element $e$ or a set $Z$ if $e \in E(M)$ or $Z \subseteq E(M)$.

Definition 2.4.1 (Seymour, in Oxley [10, Page 481]). A set $\mathscr{M}$ of matroids is 2-rounded if every member of $\mathscr{M}$ is 3-connected and the following condition holds: If $M$ is a 3 -connected matroid with a minor in $\mathscr{M}$ and $Z$ is a 2-element subset of $E(M)$, then $M$ has a minor from $\mathscr{M}$ using $Z$.

We will use the following results at various locations in this thesis.

Lemma 2.4.2 (Seymour, Theorem $3.1[18]$ ). If $x$ and $y$ are elements of a non-binary 3-connected matroid $M$, then $M$ has a $U_{2,4}$ minor using both $x$ and $y$. 


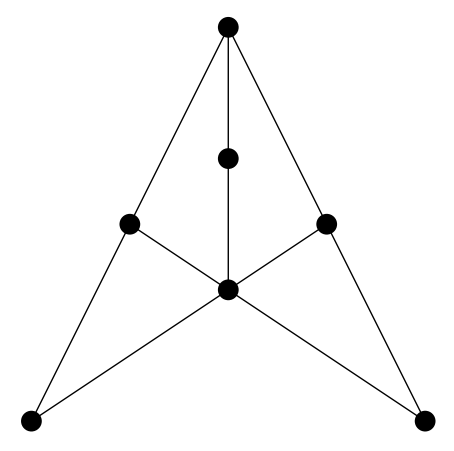

Figure 2.20: $F_{7}^{=}$

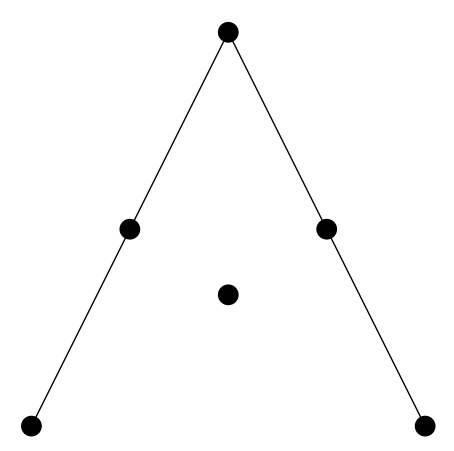

Figure 2.21: $Q_{6}$

Lemma 2.4.3. Within the class of golden-mean but non-ternary matroids, the set $\left\{U_{2,5}, U_{3,5}, F_{7}^{=},\left(F_{7}^{=}\right)^{*}, Q_{6}\right\}$ is a 2-rounded set of matroids.

Proof. We prove this by a computer check with Sage, using [10, Theorem 12.3.9]. The code is in Appendix 2.A.5

Geometric representation of the matroids $F_{7}^{=}$and $Q_{6}$ are given in Figure 2.20 and Figure 2.21, respectively.

\section{Appendix 2.A Code}

All code is written in Python for Sage [19], in particular the matroids package [14]. In order to get it to run, one may need to import the advanced matroid functions: from sage.matroids.advanced import *.

\section{A.1 Universal Variables}


funds $=[0,1,4,5,6,14,15,16]$

The allowed cross-ratios for golden-mean $G F(19)$ matrices are stored in funds.

\section{A.2 Universal Functions}

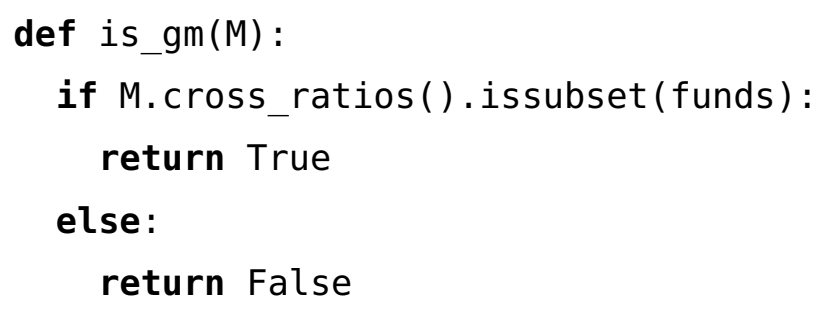

This function, is_gm( ), returns True if the given $G F(19)$-matrix represents a golden-mean matroid and False otherwise.

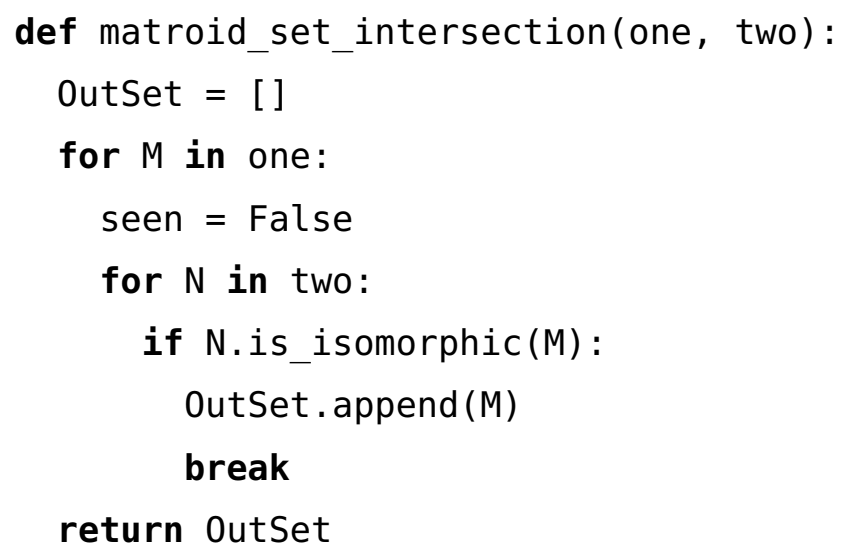

This function, matroid_set_intersection( ), takes two lists, one and two, of matroids and returns the matroids from one that are isomorphic to a matroid in two.

\section{A.3 Lemma 2.1.11}

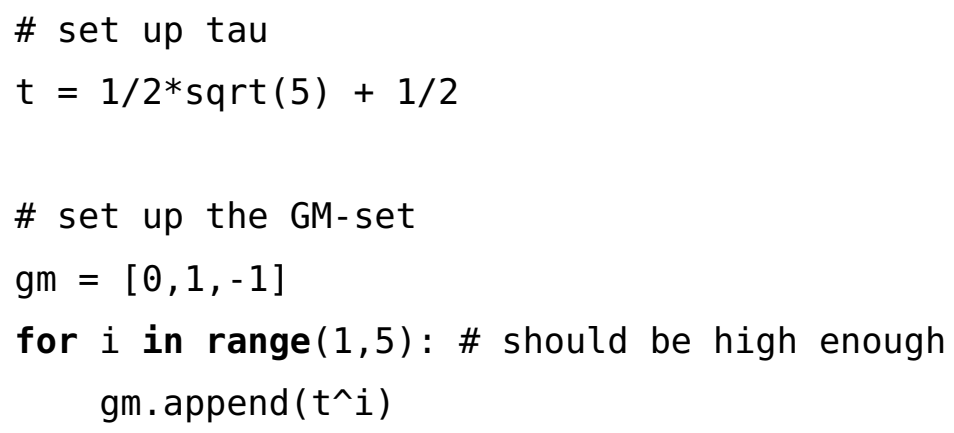




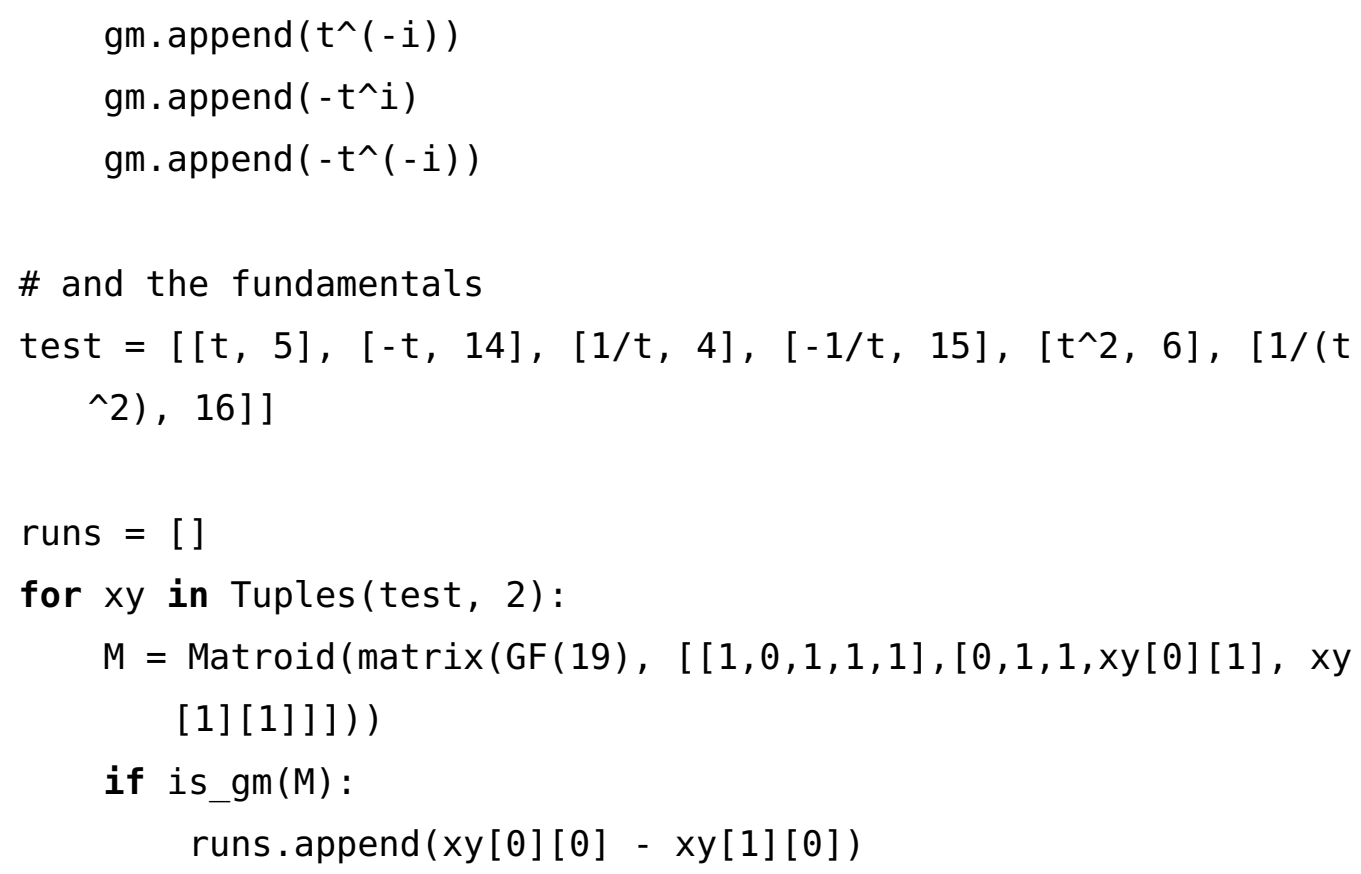

This code starts by constructing $\left\{ \pm \tau^{i} \mid i \in \mathbb{Z}\right\}$ for $i \in\{-5,-4, \ldots, 4,5\}$, and storing these values in $\mathrm{gm}$. The list test contains tuples of the form $(\psi(x), x)$, for relevant $x$. These values come from Table 2.1. We then form $\mathfrak{N}$ over $G F(19)$, and find out which values of $p$ and $q$ give us a matroid $M$ with cross-ratios in $S$. We then compute $\psi(p)-\psi(q)$ and check that this is a member of gm, proving the claim in the lemma.

\section{A.4 Lemma 2.3.1}

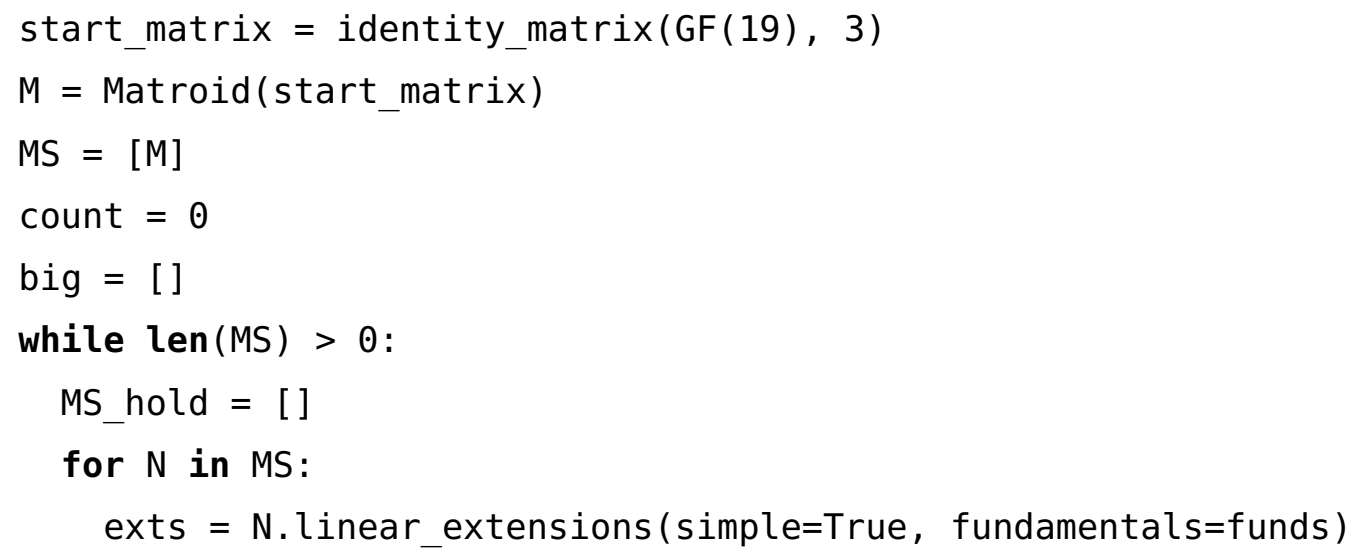




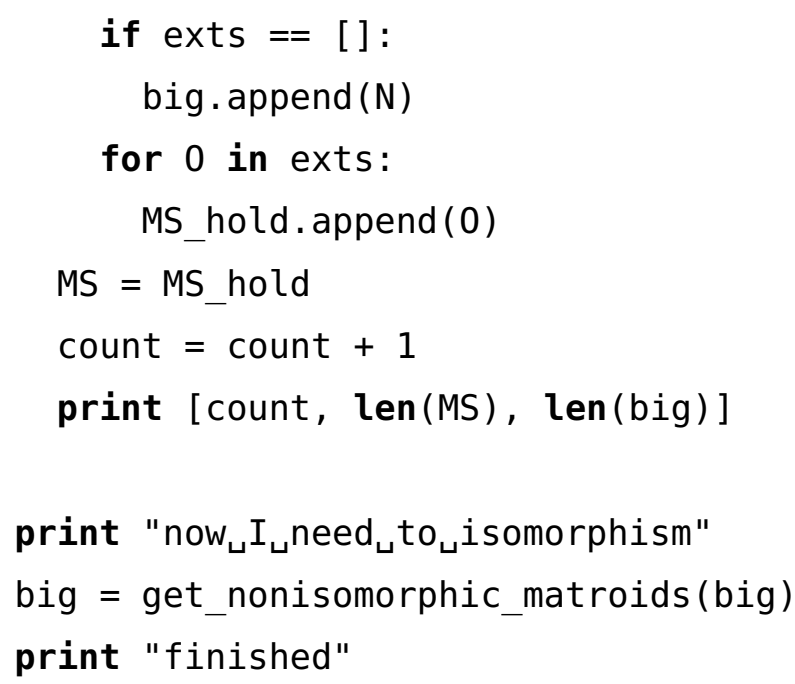

This code starts from an identity matrix and builds all simple golden-mean matroids (over $G F(19)$ ) of rank three. If a matroid appears that has no extensions, then this matroid is extremal, and we store it in the big list. Once there are no more possible extensions, we have stored all extremal rankthree golden-mean matroids in big, so we get one copy of each isomorphism class.

Upon running this code (which takes a long time), we discover the matroids listed in Lemma 2.3.1.

\section{A.5 Lemma 2.4.3}

\# set up the matroids

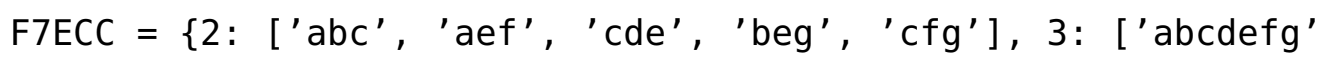
]\}

$\mathrm{U} 25=$ matroids. Uniform $(2,5)$

Q6 = matroids.named_matroids.Q6()

F7E $=$ Matroid (groundset='abcdefg', circuit_closures $=$ F7ECC)

\# and the duals (except Q6)

$\mathrm{U} 25 \mathrm{~d}=\mathrm{U} 25$.dual ()

F7Ed $=$ F7E.dual ()

ThreeRounded $=[\mathrm{U} 25, \mathrm{U} 25 \mathrm{~d}, \mathrm{Q6}, \mathrm{F} 7 \mathrm{E}, \mathrm{F} 7 \mathrm{Ed}]$ 
\# since we don't have unique representation, get all the representations.

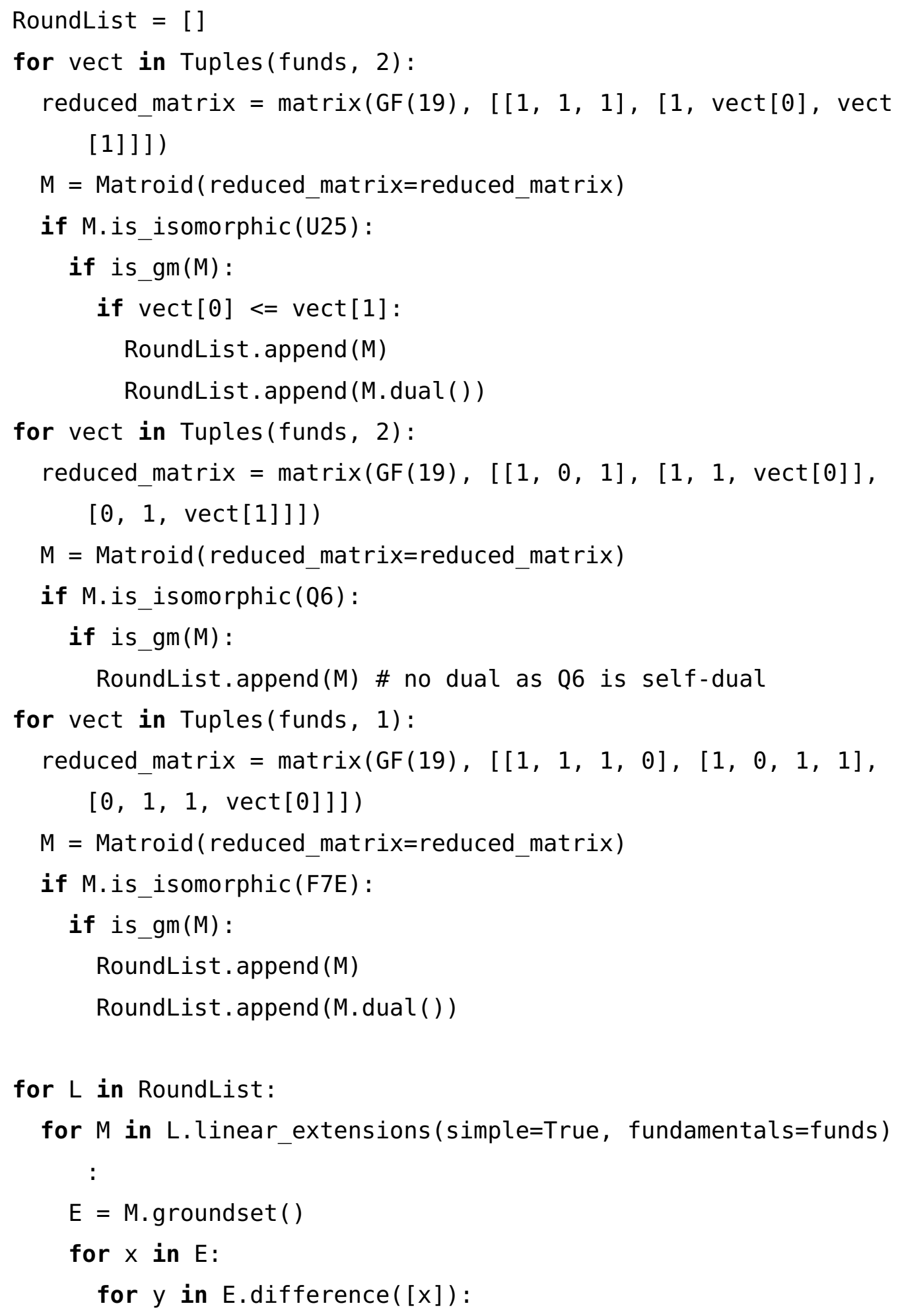




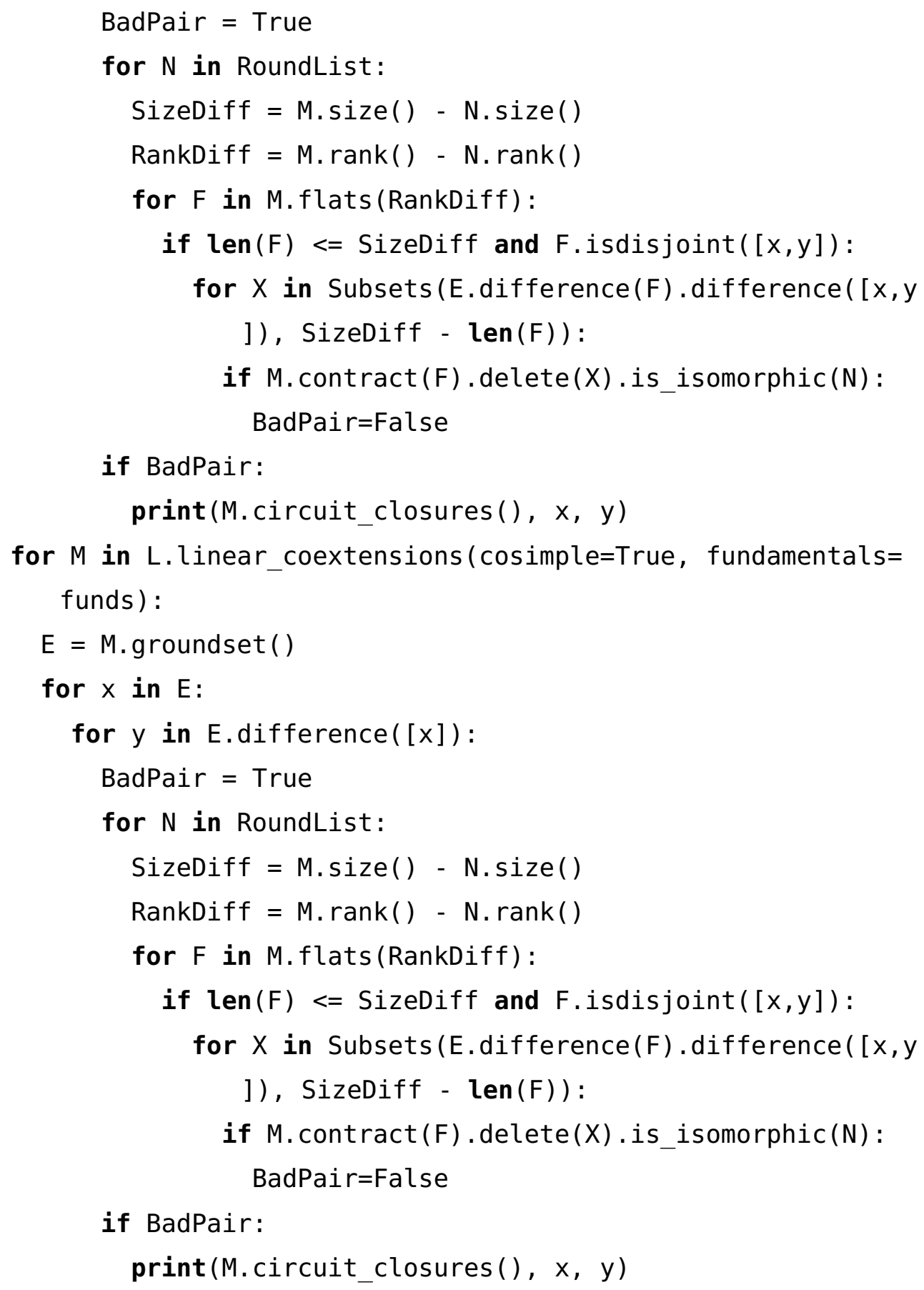

This code proves Lemma 2.4.3. It starts by constructing all possible $G F(19)$ representations of $U_{2,5}, Q_{6}$, and $F_{7}^{=}$, and, where appropriate, their duals. We then construct all single-element extensions of these matroids, and try to place all subsets of size two into one of these matroids. 
If you plug in a number and the math starts getting creepy (anything involving fractions or negative numbers is creepy)...

Doug Pierce

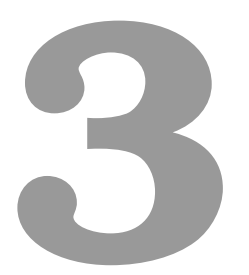

Connectivity

In this chapter we prove that any minimal counterexample to Conjecture 1.2.3 without a $B R$ minor is vertically 4 -connected. We will use this result later in the thesis.

\section{$3.1 \quad 3$-connectivity}

Definition 3.1.1. Let $M=(E, r)$ be a matroid and let $k>1$ be an integer. A $\boldsymbol{k}$-separation of $M$ is a partition $(X, Y)$ of $E$ with the property that $|X|,|Y| \geq k$, and $r(X)+r(Y)-r(M)<k$. The separation is an exact $\boldsymbol{k}$-separation if $r(X)+r(Y)-r(M)=k-1$. If $M$ has no $n$-separations for all $n \leq k$, then $M$ is $(\boldsymbol{k}+\mathbf{1})$-connected.

Recall the function $h(r)$ from Section 1.2:

$$
h(r)= \begin{cases}\left(\begin{array}{c}
r+3 \\
2
\end{array}\right)-5 & \text { if } r \neq 3 \\
11 & \text { if } r=3\end{cases}
$$


Lemma 3.1.2. Let $M$ be a rank-r simple golden-mean matroid such that $\epsilon(M) \geq h(r)$. If all proper minors $N$ of $M$ have the property that $\epsilon(N) \leq$ $h(r)$, then $M$ is 2-connected.

Proof. If $M$ has rank three, then, by Lemma 2.3.1, this is obviously true. Hence $M$ must have rank at least four.

Assume that $M$ is not 2-connected. Then there exists an exact 1-separation $\left(X_{1}, X_{2}\right)$ of $M$. Let $r\left(X_{i}\right)=r_{i}$ for $i \in\{1,2\}$. As $M \mid X_{i}$ is a proper minor of $M, \epsilon\left(M \mid X_{i}\right)$ can be no larger than $\left(\begin{array}{c}r_{i}+3 \\ 2\end{array}\right)-4$, for $i \in\{1,2\}$. Note that this is not the definition of $h(r)$, as we need to consider the case where $r_{i}=3$. So

$$
\begin{aligned}
\epsilon(M) & =\epsilon\left(M \mid X_{1}\right)+\epsilon\left(M \mid X_{2}\right) \\
& \leq\left(\begin{array}{c}
r_{1}+3 \\
2
\end{array}\right)+\left(\begin{array}{c}
r_{2}+3 \\
2
\end{array}\right)-8 \\
& =\frac{1}{2}\left(r_{1}^{2}+r_{2}^{2}+5 r_{1}+5 r_{2}-4\right) .
\end{aligned}
$$

Also,

$$
\begin{aligned}
\epsilon(M) & \geq\left(\begin{array}{c}
r+3 \\
2
\end{array}\right)-5 \\
& =\left(\begin{array}{c}
r_{1}+r_{2}+3 \\
2
\end{array}\right)-5 \\
& =\frac{1}{2}\left(r_{1}^{2}+r_{2}^{2}+5 r_{1}+5 r_{2}+2 r_{1} r_{2}-4\right) .
\end{aligned}
$$

Combining (3.1.1) and (3.1.2), we get

$$
\begin{aligned}
\frac{1}{2}\left(r_{1}^{2}+r_{2}^{2}+5 r_{1}+5 r_{2}-4\right) & \geq \frac{1}{2}\left(r_{1}^{2}+r_{2}^{2}+5 r_{1}+5 r_{2}+2 r_{1} r_{2}-4\right) \\
0 & \geq 2 r_{1} r_{2} .
\end{aligned}
$$

As both $r_{1}$ and $r_{2}$ are positive, this is a contradiction. Therefore $M$ is 2connected. 
Lemma 3.1.3. Let $M$ be a rank-r simple golden-mean matroid such that $\epsilon(M) \geq h(r)$. If all proper minors $N$ of $M$ have the property that $\epsilon(N) \leq$ $h(r)$, then $M$ is 3-connected.

Proof. If $M$ has rank three, then, by Lemma 2.3.1, this is obviously true. Hence $M$ must have rank at least four.

Assume that $M$ is not 3-connected. Then, by Lemma 3.1.2, $M$ is 2-connected, so there are no exact 1-separations. Hence there exists an exact 2-separation $\left(X_{1}, X_{2}\right)$ of $M$. Let $r\left(X_{i}\right)=r_{i}$. As $M_{i}=M \mid X_{i}$ is a proper minor of $M$, it follows that $\epsilon\left(M_{i}\right)$ can be no larger than $\left(\begin{array}{c}r_{i}+3 \\ 2\end{array}\right)-4$, for $i \in\{1,2\}$. Note that this is not the definition of $h(r)$, as we need to consider the case where $r_{i}=3$. So

$$
\begin{aligned}
\epsilon(M) & =\epsilon\left(M_{1}\right)+\epsilon\left(M_{2}\right) \\
& \leq\left(\begin{array}{c}
r_{1}+3 \\
2
\end{array}\right)+\left(\begin{array}{c}
r_{2}+3 \\
2
\end{array}\right)-8 \\
& =\frac{1}{2}\left(r_{1}^{2}+r_{2}^{2}+5 r_{1}+5 r_{2}-4\right) .
\end{aligned}
$$

Also,

$$
\begin{aligned}
\epsilon(M) & \geq\left(\begin{array}{c}
r+3 \\
2
\end{array}\right)-5 \\
& =\left(\begin{array}{c}
\left(r_{1}+r_{2}-1\right)+3 \\
2
\end{array}\right)-5 \\
& =\frac{1}{2}\left(r_{1}^{2}+r_{2}^{2}+3 r_{1}+3 r_{2}+2 r_{1} r_{2}-8\right) .
\end{aligned}
$$

Combining (3.1.3) and (3.1.4), we get

$$
\begin{aligned}
\frac{1}{2}\left(r_{1}^{2}+r_{2}^{2}+5 r_{1}+5 r_{2}-4\right) & \geq \frac{1}{2}\left(r_{1}^{2}+r_{2}^{2}+3 r_{1}+3 r_{2}+2 r_{1} r_{2}-8\right) \\
2 r_{1}+2 r_{2} & \geq 2 r_{1} r_{2}-4 \\
r_{1}+r_{2} & \geq r_{1} r_{2}-2
\end{aligned}
$$


If $r_{i}=1$, then $M$ contains a parallel class, and is therefore not simple, contradicting the definition of $M$.

If $r_{1}=r_{2}=2$, then $r(M)=3$, and this case has already been covered.

Hence, without loss of generality, $r_{1}=3$ and $r_{2}=2$, and $r(M)=4$. Then $M$ must have at least sixteen elements, and so $M_{1} \cong B R$ and $M_{2} \cong U_{2,5}$. If $E\left(M_{1}\right) \cap E\left(M_{2}\right)$ is empty, we would be able to find a $U_{2,6}$-minor of $M$, and so would have at most $11+5-1=15$ elements in $M$, meaning $\epsilon(M)<h(r)$, contradicting the definition of $M$.

Therefore $M$ is 3-connected.

\subsection{Vertical Connectivity}

We first define what it means for a matroid to be vertically 4-connected.

Definition 3.2.1. Let $M=(E, r)$ be a 3-connected matroid. The exact 3-separation $(X, Y)$ of $E$ is an exact vertical 3-separation if $r(X)$ and $r(Y)$ are both at least three.

Definition 3.2.2. Let $M=(E, r)$ be a 3-connected matroid. If every 3separation $(X, Y)$ of $M$ has the property that $\min \{r(X), r(Y)\} \leq 2$, then $M$ is vertically 4-connected.

The following result is well known. A proof is given here for completeness.

Lemma 3.2.3. Let $M$ be a vertically 4-connected matroid, and let e $\in E(M)$ be an element of $M$. Then $\operatorname{si}(M / e)$ is 3-connected.

Proof. First, we will show that $\operatorname{si}(M / e)$ is 2-connected.

Sublemma 3.2.3.1. The matroid $\operatorname{si}(M / e)$ is 2-connected.

Subproof. Assume that $\operatorname{si}(M / e)$ is not 2-connected. Then there exists a 1separation, $\left(X_{1}^{\prime \prime}, X_{2}^{\prime \prime}\right)$ of $\operatorname{si}(M / e)$. This induces a 1-separation, $\left(X_{1}^{\prime}, X_{2}^{\prime}\right)$ of $M / e$. We now consider what happens to this partition in $M$. Let $\left(X_{1}, X_{2}\right)$ be this partition in $M$. Without loss of generality, we can assume that 
$e \in X_{1}$. Then $r_{M}\left(X_{1}\right)-r_{M / e}\left(X_{1}\right)=1$, and $r_{M}\left(X_{2}\right)-r_{M / e}\left(X_{2}\right)$ is at most one. If $r_{M}\left(X_{1}\right)-r_{M / e}\left(X_{1}\right)=r_{M}\left(X_{2}\right)-r_{M / e}\left(X_{2}\right)=1$, then

$$
r_{M}\left(X_{1}\right)+r_{M}\left(X_{2}\right)-r_{M}(M)=1
$$

Therefore $\left(X_{1}, X_{2}\right)$ is a 2 -separation of $M$, contradicting the fact that $M$ is 3-connected. Hence si $(M / e)$ must be 2-connected.

Now assume that $\operatorname{si}(M / e)$ is not 3 -connected. Then there exists a 2separation, $\left(X_{1}^{\prime \prime}, X_{2}^{\prime \prime}\right)$ of $\operatorname{si}(M / e)$. By putting back parallel elements and coloops, there is a 2-separation, $\left(X_{1}^{\prime}, X_{2}^{\prime}\right)$ of $M / e$. We now consider what happens to this 2-separation in $M$. Let $\left(X_{1}, X_{2}\right)$ be this 2-separation in $M$. If $r_{M}\left(X_{1}\right)=r_{M / e}\left(X_{1}^{\prime}\right)+1$ and $r_{M}\left(X_{2}\right)=r_{M / e}\left(X_{2}^{\prime}\right)$, then $\left(X_{1}, X_{2}\right)$ is a 2-separation of $M$, contradicting the fact that $M$ is 3 -connected. Hence $r_{M}\left(X_{1}\right)=r_{M / e}\left(X_{1}^{\prime}\right)+1$ and $r_{M}\left(X_{2}\right)=r_{M / e}\left(X_{2}^{\prime}\right)+1$. Then

$$
\begin{aligned}
r_{M}\left(X_{1}\right)+r_{M}\left(X_{2}\right)-r_{M}(M) & =r_{M / e}\left(X_{1}^{\prime}\right)+r_{M / e}\left(X_{2}^{\prime}\right)-\left(r_{M / e}(M / e)+1\right)+2 \\
& =1-1+2 \\
& =2 .
\end{aligned}
$$

So $\left(X_{1}, X_{2}\right)$ is a 3 -separation of $M$. However, in si $(M / e)$, the rank of $X_{1}^{\prime \prime}$ and the rank of $X_{2}^{\prime \prime}$ are both at least two, as it is simple. So in $M$ the rank of $X_{1}$ and the rank of $X_{2}$ are both at least three. This is a contradiction to $M$ being vertically 4 -connected, so $\operatorname{si}(M / e)$ must be 3-connected.

For our applications we only need to consider golden-mean matroids with no $B R$ minor.

Theorem 3.2.4. Let $M$ be a rank-r simple golden-mean matroid with no BR-minor such that $\epsilon(M) \geq\left(\begin{array}{c}r+3 \\ 2\end{array}\right)-5$. If all proper minors $N$ of $M$ have the property that $\epsilon(N) \leq\left(\begin{array}{c}r(N)+3 \\ 2\end{array}\right)-5$, then $M$ is vertically 4-connected.

Proof. Let $P=P G(r-1,4)$. By Lemma 3.1 .3 we know that $M$ is 3 -connected and ergo, if the lemma fails, $M$ must have an exact vertical 3-separation $\left(X_{1}, X_{2}\right)$. View $M$ as a restriction of $P$. Now,

$$
r\left(\operatorname{cl}_{P}\left(X_{1}\right) \cap \operatorname{cl}_{P}\left(X_{2}\right)\right) \leq r\left(\operatorname{cl}_{P}\left(X_{1}\right)\right)+r\left(\operatorname{cl}_{P}\left(X_{2}\right)\right)-r\left(\operatorname{cl}_{P}\left(X_{1}\right) \cup \operatorname{cl}_{P}\left(X_{2}\right)\right)
$$




$$
\begin{aligned}
& \leq r\left(X_{1}\right)+r\left(X_{2}\right)-r\left(X_{1} \cup X_{2}\right) \\
& =r\left(X_{1}\right)+r\left(X_{2}\right)-r(M) \\
& =2 .
\end{aligned}
$$

So the closures of $X_{1}$ and $X_{2}$ in $P$ meet in a line $L$ of $P$. Let $r_{i}=r\left(X_{i}\right)$ for each $i \in\{1,2\}$. As $\left(X_{1}, X_{2}\right)$ is a vertical 3-separation of $M$, both $r_{1}$ and $r_{2}$ must be at least three.

We consider $|L \cap E(M)|$, noting that it is at most five, as this is the maximum line length in a $G F(4)$-representable matroid. The strategy of the proof is to consider, for each $i \in\{1,2\}$, a simple rank- $r_{i}$ minor $M_{i}$ of $M$, obtained by deleting and contracting elements from the complement of $X_{i}$, that is spanned by $X_{i}$, contains $\left(X_{1} \cup X_{2}\right) \cap L$, and has the maximum number of points among such minors. Thus, for $\{i, j\}=\{1,2\}, M_{i}$ is obtained from $M$ by contracting elements in $X_{j}$ so that as many points in $X_{j}$ as possible are projected into the span of $X_{i}$. Clearly we may view $M_{i}$ as a restriction of $P \mid\left(L \cup X_{i}\right)$.

Now

$$
\begin{aligned}
\epsilon(M)= & \left|X_{1}\right|+\left|X_{2}\right| \\
= & \left(\epsilon\left(M_{1}\right)-\left|\left(E\left(M_{1}\right) \cap L\right)-X_{1}\right|\right) \\
& +\left(\epsilon\left(M_{2}\right)-\left|\left(E\left(M_{2}\right) \cap L\right)-X_{2}\right|\right) .
\end{aligned}
$$

As $M_{i}$ is a proper minor of $M, \epsilon\left(M_{i}\right)$ can be no larger than $\left(\begin{array}{c}r_{i}+3 \\ 2\end{array}\right)-5$, for $i \in\{1,2\}$. Thus

$$
\begin{aligned}
\epsilon(M) \leq & \left(\begin{array}{c}
r_{1}+3 \\
2
\end{array}\right)+\left(\begin{array}{c}
r_{2}+3 \\
2
\end{array}\right)-10 \\
& -\left(\left|\left(E\left(M_{1}\right) \cap L\right)-X_{1}\right|+\left|\left(E\left(M_{2}\right) \cap L\right)-X_{2}\right|\right) .
\end{aligned}
$$

Also,

$$
\epsilon(M) \geq\left(\begin{array}{c}
\left(r_{1}+r_{2}-2\right)+3 \\
2
\end{array}\right)-5
$$


So

$$
\begin{aligned}
\frac{1}{2}\left(r_{1}+r_{2}\right)\left(r_{1}+r_{2}+1\right) \leq & \frac{1}{2}\left(\left(r_{1}+2\right)\left(r_{1}+3\right)+\left(r_{2}+2\right)\left(r_{2}+3\right)\right)-5 \\
& -\left(\left|\left(E\left(M_{1}\right) \cap L\right)-X_{1}\right|+\left|\left(E\left(M_{2}\right) \cap L\right)-X_{2}\right|\right) .
\end{aligned}
$$

Expanding out gives

$$
\begin{aligned}
\frac{1}{2}\left(r_{1}^{2}+r_{2}^{2}+2 r_{1} r_{2}+r_{1}+r_{2}\right) \leq & \frac{1}{2}\left(r_{1}^{2}+r_{2}^{2}+5 r_{1}+5 r_{2}+2\right) \\
& -\left|\left(E\left(M_{1}\right) \cap L\right)-X_{1}\right|-\left|\left(E\left(M_{2}\right) \cap L\right)-X_{2}\right| .
\end{aligned}
$$

Hence

$$
r_{1} r_{2}-2 r_{1}-2 r_{2}-1 \leq-\left(\left|\left(E\left(M_{1}\right) \cap L\right)-X_{1}\right|+\left|\left(E\left(M_{2}\right) \cap L\right)-X_{2}\right|\right) .
$$

And so

$$
\left(r_{1}-2\right)\left(r_{2}-2\right) \leq 5-\left(\left|E\left(M_{1}\right) \cap L\right|-\left|X_{1} \cap L\right|+\left|E\left(M_{2}\right) \cap L\right|-\left|X_{2} \cap L\right|\right) .
$$

But

$$
\begin{aligned}
\left|E\left(M_{i}\right) \cap L\right| & \geq\left|\left(X_{1} \cup X_{2}\right) \cap L\right| \\
& =\left|X_{1} \cap L\right|+\left|X_{2} \cap L\right|,
\end{aligned}
$$

so, for each $i \in\{1,2\}$,

$$
\left(r_{1}-2\right)\left(r_{2}-2\right) \leq 5-\left|E\left(M_{i}\right) \cap L\right|
$$

Next we take a basis $B_{1}$ for $X_{1}$ and extend it to a basis $B$ for $M$. Then $\left|B-B_{1}\right|=r(M)-r\left(X_{1}\right)=r\left(X_{2}\right)-2$. It follows that $r_{M /\left(B-B_{1}\right)}\left(X_{2}-B\right)=2$. This means that we can always project at least two points from $X_{2}$ into the span of $X_{1}$ by contracting only points in $X_{2}-X_{1}$. Hence it can be assumed that $M_{1}$ satisfies

$$
\left|E\left(M_{1}\right) \cap L\right| \geq 2 .
$$


Similarly,

$$
\left|E\left(M_{2}\right) \cap L\right| \geq 2
$$

Combining (3.2.4) and (3.2.5) with (3.2.3), we get

$$
\left(r_{1}-2\right)\left(r_{2}-2\right) \leq 3
$$

If $r_{1}$ and $r_{2}$ are both at least four, then (3.2.6) is a contradiction. Therefore, we can assume that $r_{1}=3$. So (3.2.6) becomes $r_{2} \leq 5$.

Now suppose $\left|\left(X_{1} \cup X_{2}\right) \cap L\right| \geq 3$.

Sublemma 3.2.4.1. If $\left|\left(X_{1} \cup X_{2}\right) \cap L\right| \geq 3$, then both $M_{1}$ and $M_{2}$ are 3connected.

Subproof. Let $M_{1}^{\prime}=M \mid\left(X_{1} \cup\left(X_{2} \cap L\right)\right)$. Note that, by definition, $X_{1}$ spans L. Now

$$
\begin{aligned}
r\left(M_{1}^{\prime}\right) & =r\left(M \mid\left(X_{1} \cup\left(X_{2} \cap L\right)\right)\right) \\
& =r\left(M \mid X_{1}\right) \\
& =r\left(X_{1}\right) .
\end{aligned}
$$

If $\left(Y_{1}, Y_{2}\right)$ is a $k$-separation of $M_{1}^{\prime}$ for some $k \leq 2$, then $r\left(Y_{1}\right)+r\left(Y_{2}\right)-r\left(X_{1}\right) \leq$ $k-1$, and, as $r\left(X_{1}\right)=r(M)-r\left(X_{2}\right)+2$,

$$
r\left(Y_{1}\right)+r\left(Y_{2}\right)-r(M)+r\left(X_{2}\right)-2 \leq k-1 .
$$

Without loss of generality, we may assume that $\left|Y_{1} \cap L\right| \geq 2$. Then

$$
\begin{aligned}
r\left(Y_{1} \cup X_{2}\right) & \leq r\left(\operatorname{cl}\left(Y_{1}\right) \cup \operatorname{cl}\left(X_{2}\right)\right) \\
& \leq r\left(\operatorname{cl}\left(Y_{1}\right)\right)+r\left(\operatorname{cl}\left(X_{2}\right)\right)-r\left(\operatorname{cl}\left(Y_{1}\right) \cap \operatorname{cl}\left(X_{2}\right)\right) \\
& \leq r\left(Y_{1}\right)+r\left(X_{2}\right)-r\left(\operatorname{cl}\left(Y_{1}\right) \cap \operatorname{cl}\left(X_{2}\right)\right) .
\end{aligned}
$$

Observe that $\operatorname{cl}\left(Y_{1}\right) \cap \operatorname{cl}\left(X_{2}\right)$ contains $L$, so $r\left(\operatorname{cl}\left(Y_{1}\right) \cap \operatorname{cl}\left(X_{2}\right)\right) \geq 2$. Hence 


$$
r\left(Y_{1} \cup X_{2}\right) \leq r\left(Y_{1}\right)+r\left(X_{2}\right)-2 .
$$

Combining (3.2.7) with (3.2.8) gives $r\left(Y_{2}\right)+r\left(Y_{1} \cup X_{2}\right)-r(M) \leq k-1$, so $\left(Y_{2},\left(Y_{1} \cup X_{2}\right)-Y_{2}\right)$ is a $k$-separation of $M$, a contradiction. Thus $M_{1}^{\prime}$ is 3-connected and, as $M_{1}$ is obtained from $M_{1}^{\prime}$ by adding elements that are not loops, coloops or in parallel classes, $M_{1}$ is also 3-connected. Similarly, $M_{2}$ is 3-connected.

Sublemma 3.2.4.2. If $\left|\left(X_{1} \cup X_{2}\right) \cap L\right| \geq 3$, then $M_{1}$ and $M_{2}$ are not binary.

Proof. If $\left|\left(X_{1} \cup X_{2}\right) \cap L\right|>3$, then it is easy to find a $U_{2,4}$-minor of both $M_{1}$ and $M_{2}$. Hence we can assume that $\left|\left(X_{1} \cup X_{2}\right) \cap L\right|=3$. From (3.2.6) we know that $r_{1}=3$ and $r_{2} \leq 5$.

Firstly, assume that $M_{1}$ is binary. Then, as $M_{1}$ is golden-mean, it is representable over $G F(5)$, and hence, by Theorem 1.1.8, it is regular. Therefore, by Theorem 1.1.9, $\epsilon\left(M_{1}\right) \leq 6$. If $r_{2}=3$, then $r=4$, and so $\epsilon(M) \geq 16$. Hence $\epsilon\left(M_{2}\right) \geq 13$, contradicting Lemma 2.3.1. Likewise, if $r_{2}=4$, then $\epsilon\left(M_{2}\right) \geq 20$, and if $r_{2}=5$, then $\epsilon(M) \geq 28$, both contradicting the definition of $M$. Hence $M_{1}$ cannot be binary.

Now assume that $M_{2}$ is binary. As $M_{1}$ is a rank-three proper minor of $M$, by the definition of $M, \epsilon\left(M_{1}\right) \leq 10$. Now consider $M_{2}$. As it is binary and representable over $G F(5)$, it is therefore regular by Theorem 1.1.8. If $r_{2}=3$, then $r=4$, and so $\epsilon(M) \geq 16$. Hence $\epsilon\left(M_{2}\right) \geq 9$. Likewise, if $r_{2}=4$, then $\epsilon\left(M_{2}\right) \geq 16$, and if $r_{2}=5$, then $\epsilon\left(M_{2}\right) \geq 24$. In all three cases, Theorem 1.1.9 is contradicted, and hence $M_{2}$ cannot be binary.

Sublemma 3.2.4.3. If $\left|\left(X_{1} \cup X_{2}\right) \cap L\right| \geq 3$, then $\left|\left(X_{1} \cup X_{2}\right) \cap L\right| \geq 4$, and $r_{1}=r_{2}=3$.

Subproof. By Sublemma 3.2.4.2, $M_{i}$ is not binary. Hence by Lemma 2.4.2, for $\{i, j\}=\{1,2\}$, the matroid $M_{i}$ has a $U_{2,4}$ minor using $\left(X_{1} \cup X_{2}\right) \cap L$, and so $\left|E\left(M_{j}\right) \cap L\right| \geq 4$.

Firstly, assume that $\left|E\left(M_{1}\right) \cap L\right|=\left|E\left(M_{2}\right) \cap L\right|=4$. Then (3.2.1) becomes

$$
r_{2} \leq 7+\left|X_{1} \cap L\right|+\left|X_{2} \cap L\right|-4-4
$$




$$
=\left|X_{1} \cap L\right|+\left|X_{2} \cap L\right|-1
$$

If $\left|X_{1} \cap L\right|+\left|X_{2} \cap L\right|<4$, then (3.2.9) becomes $r_{2} \leq 2$, contradicting the fact that $\left(X_{1}, X_{2}\right)$ is a vertical 3-separation of $M$.

If $\left|X_{1} \cap L\right|+\left|X_{2} \cap L\right|=5$, then $\left|E\left(M_{1}\right) \cap L\right|=\left|E\left(M_{2}\right) \cap L\right|=5$, and (3.2.3) implies that $r_{1}, r_{2} \leq 2$, contradicting the fact that $\left(X_{1}, X_{2}\right)$ is a vertical 3-separation of $M$.

Hence $\left|X_{1} \cap L\right|+\left|X_{2} \cap L\right|=4$, and (3.2.9) becomes $r_{2} \leq 3$, and the fact that $\left(X_{1}, X_{2}\right)$ is a vertical 3 -separation of $M$ implies that $r_{2}=3$.

Next, assume that $\left|E\left(M_{1}\right) \cap L\right|=4$ and $\left|E\left(M_{2}\right) \cap L\right|=5$. Then (3.2.1) becomes

$$
\begin{aligned}
r_{2} & \leq 7+\left|X_{1} \cap L\right|+\left|X_{2} \cap L\right|-4-5 \\
& =\left|X_{1} \cap L\right|+\left|X_{2} \cap L\right|-2 .
\end{aligned}
$$

Because $L$ is a line of the projective geometry $P G(r-1,4)$, it contains at most five elements. Hence $\left|X_{1} \cap L\right|+\left|X_{2} \cap L\right| \leq 5$, and so (3.2.10) becomes $r_{2} \leq 3$, and the fact that $\left(X_{1}, X_{2}\right)$ is a vertical 3-separation of $M$ implies that $r_{2}=3$.

Lastly, assume that $\left|E\left(M_{1}\right) \cap L\right|=\left|E\left(M_{2}\right) \cap L\right|=5$. Then (3.2.1) becomes

$$
\begin{aligned}
r_{2} & \leq 7+\left|X_{1} \cap L\right|+\left|X_{2} \cap L\right|-5-5 \\
& =\left|X_{1} \cap L\right|+\left|X_{2} \cap L\right|-3 .
\end{aligned}
$$

As $L$ is a line of the projective geometry $P G(r-1,4)$, it contains exactly five elements. Hence $\left|X_{1} \cap L\right|+\left|X_{2} \cap L\right| \leq 5$, and so (3.2.11) becomes $r_{2} \leq 2$, contradicting the fact that $\left(X_{1}, X_{2}\right)$ is a vertical 3-separation of $M$.

So $3 \leq\left|X_{1} \cap L\right|+\left|X_{2} \cap L\right|=\left|\left(X_{1} \cup X_{2}\right) \cap L\right| \leq 4$, and $r_{1}=r_{2}=3$.

We will now show that $\left|\left(X_{1} \cup X_{2}\right) \cap L\right|$ can be neither three nor four. Note that (3.2.3) implies that $\left|\left(X_{1} \cup X_{2}\right) \cap L\right| \neq 5$.

Sublemma 3.2.4.4. $\left|\left(X_{1} \cup X_{2}\right) \cap L\right| \neq 4$. 
Subproof. If $\left|\left(X_{1} \cup X_{2}\right) \cap L\right|=4$, then, as $M \mid X_{i}$ is a proper rank-three minor of $M$, it has at most ten elements. Furthermore, as $M$ has rank four, $\epsilon(M) \geq$ 16. Hence $\left|X_{1}-L\right|=\left|X_{2}-L\right|=6$.

Therefore, for $i \in 1,2$, we can see that $\epsilon\left(M \mid\left(X_{i} \cup L\right)\right)=10$. So, by Lemma 2.3.1, $M \mid\left(X_{i} \cup L\right)$ is isomorphic to one of $H P_{3}, B R \backslash p, Y_{10}, S_{10}$, $T_{3}^{2}$, or $G_{3}$.

However, the matroids $\mathrm{HP}_{3}$ and $\mathrm{T}_{3}^{2}$ have no line of exactly four points, so it is not possible for either of them to be one of the $M \mid\left(X_{i} \cup L\right)$ restrictions. Hence $M$ is two (not necessarily distinct) matroids, $N_{1}$ and $N_{2}$, from the set $\left\{S_{10}, B R \backslash p, Y_{10}, G_{3}\right\}$ identified along a four-point line. No matter what $N_{1}$ is, it is easy to see that an extra point can be projected onto $L$, meaning that $\left|E\left(M_{2}\right) \cap L\right|=5$, contradicting (3.2.3).

Hence $\left|\left(X_{1} \cup X_{2}\right) \cap L\right| \neq 4$.

Sublemma 3.2.4.5. $\left|\left(X_{1} \cup X_{2}\right) \cap L\right| \neq 3$.

Subproof. If $\left|\left(X_{1} \cup X_{2}\right) \cap L\right|=3$, then, as $M \mid X_{i}$ is a proper rank-three minor of $M$, it has at most ten elements. Furthermore, as $M$ has rank four it must have at least sixteen elements. Hence we can assume that $\left|X_{1} \cup L\right|=7$ and $\left|X_{2} \cup L\right| \geq 6$.

Therefore $M$ is isomorphic to a member of $\left\{H P_{3}, T_{3}^{2}, G_{3}, S_{10}, B R \backslash p, Y_{10}\right\}$ identified along a three-point line with a matroid of at least 9 elements. In all cases, it is easy to see that we can contract two points from $M \mid\left(X_{1} \cup L\right)$ onto $L$, giving $\left|E\left(M_{2}\right) \cap L\right|=5$, contradicting (3.2.3).

Hence $\left|\left(X_{1} \cup X_{2}\right) \cap L\right| \leq 2$. Then (3.2.1) becomes

$$
3 \leq r_{2} \leq 7-\left|E\left(M_{1}\right) \cap L\right|-\left|E\left(M_{2}\right) \cap L\right|+\left|\left(X_{1} \cup X_{2}\right) \cap L\right| .
$$

We will now show that there is no possible value for $\left|\left(X_{1} \cup X_{2}\right) \cap L\right|$.

Sublemma 3.2.4.6. $\left|\left(X_{1} \cup X_{2}\right) \cap L\right| \neq 0$.

Subproof. Assume that $\left|\left(X_{1} \cup X_{2}\right) \cap L\right|=0$. From (3.2.4) and (3.2.5) we know that both $\left|E\left(M_{1}\right) \cap L\right|$ and $\left|E\left(M_{2}\right) \cap L\right|$ are at least two. Combining this information with (3.2.12), we see that $r_{2}=3$ and both $\left|E\left(M_{1}\right) \cap L\right|$ and 
$\left|E\left(M_{2}\right) \cap L\right|$ must be exactly two. By assumption, $\epsilon(M) \geq 16$, so, without loss of generality, $\left|X_{2}\right| \geq 8$. As $M \mid\left(X_{2}\right)$ is $G F(5)$-representable, if $M \mid\left(X_{2}\right)$ has no $U_{2,4}$-minor, then it is regular, and so it must be no larger than $M\left(K_{4}\right)$. If $M \mid\left(X_{2}\right)$ has a $U_{2,4}$-minor, then it is possible to contract a point from $M \mid\left(X_{2}\right)$ and put four points on $L$, so $\left|E\left(M_{1}\right) \cap L\right|=4$, which is a contradiction to $\left|E\left(M_{1}\right) \cap L\right|$ being equal to two. Hence $\epsilon\left(M \mid\left(X_{2}\right)\right) \leq 6$, a contradiction to $\left|X_{2}\right| \geq 8$. So $\left|\left(X_{1} \cup X_{2}\right) \cap L\right|$ cannot equal zero.

Sublemma 3.2.4.7. $\left|\left(X_{1} \cup X_{2}\right) \cap L\right| \neq 1$.

Subproof. Assume that $\left|\left(X_{1} \cup X_{2}\right) \cap L\right|=1$, and suppose that $r_{2}=3$. Then $r=4$. Then, by (3.2.4), (3.2.5), and (3.2.12), we see that $2 \leq\left|E\left(M_{i}\right) \cap L\right| \leq$ 3 , for $i \in\{1,2\}$. So, as $M$ has rank four, it must have at least sixteen elements. So $\left|X_{1} \cup X_{2}\right| \geq 16$. Hence, without loss of generality, $\left|X_{1}\right| \geq$ 8. Now pick $x \in X_{1}-L$. As $\left|E\left(M_{2}\right) \cap L\right| \leq 3$, there can be no more than three lines passing through $x$. Hence there must be at least one fourpoint line containing $x$. Now contract an element not on this line, giving $\left|E\left(M_{2}\right) \cap L\right|=4$, a contradiction. So $r_{2} \neq 3$. From (3.2.4) and (3.2.5), we know that both $\left|E\left(M_{1}\right) \cap L\right|$ and $\left|E\left(M_{2}\right) \cap L\right|$ are at least two. Combining this information with (3.2.12), we see that $r_{2}=4$, and $\left|E\left(M_{i}\right) \cap L\right|=2$, for $i \in\{1,2\}$. So $r=5$, implying that $M$ has at least 23 elements. So $\left|X_{1} \cup X_{2}\right| \geq 23$. Also, $\epsilon\left(M \mid X_{2}\right)$ can be no larger than sixteen, so $\left|X_{2}\right| \leq 16$. Therefore $\left|X_{1}\right| \geq 7$. Now pick $x \in X_{1}-L$. Because $\left|E\left(M_{2}\right) \cap L\right|=2$, it follows that $x$ is on at most two lines. Therefore, one of these lines has at least four points and we can contract an element on the other line to get $\left|E\left(M_{2}\right) \cap L\right|=4$, a contradiction. So $\left|\left(X_{1} \cup X_{2}\right) \cap L\right|$ cannot equal one.

Sublemma 3.2.4.8. $\left|\left(X_{1} \cup X_{2}\right) \cap L\right| \neq 2$.

Subproof. Assume $\left|\left(X_{1} \cup X_{2}\right) \cap L\right|=2$. So (3.2.12) becomes

$$
3 \leq r_{2} \leq 9-\left|E\left(M_{1}\right) \cap L\right|-\left|E\left(M_{2}\right) \cap L\right|
$$

Let $\{1,2\}=\{i, j\}$. Assume that $\left|E\left(M_{j}\right) \cap L\right|=2$, and let $E\left(M_{j}\right) \cap L=\{s, t\}$. Then pick $x \in X_{i}-L$. As $\left|E\left(M_{j}\right) \cap L\right|=2$, everything in $X_{i}$ must be on a line with $x$ and either $s$ or $t$. So $r_{i}=3$. Hence $r_{j}$ can be 3,4 , or 5 . If $r_{j}=3$, then, as $M$ has rank four, $\epsilon(M) \geq 16$, so $\left|X_{j} \cup\left(\left(X_{1} \cup X_{2}\right) \cap L\right)\right| \leq 10$, 
and therefore $\left|X_{i} \cup\left(\left(X_{1} \cup X_{2}\right) \cap L\right)\right| \geq 8$. Using the same reasoning, we see that if $r_{j}=4$, then $\left|X_{i} \cup\left(\left(X_{1} \cup X_{2}\right) \cap L\right)\right| \geq 9$, and if $r_{j}=5$, then $\left|X_{i} \cup\left(\left(X_{1} \cup X_{2}\right) \cap L\right)\right| \geq 10$. In all three cases, both lines through $x$ must have at least three points on them, and we contract a point from $X_{i}-\{x, s, t\}$ to project at least three points onto $L$, implying that $\left|E\left(M_{j}\right) \cap L\right| \geq 3$, a contradiction.

So now it follows from (3.2.4), (3.2.5), and (3.2.13) that $\left|E\left(M_{1}\right) \cap L\right|=$ $\left|E\left(M_{2}\right) \cap L\right|=3$, and $r_{2}=3$. As $M$ has rank four, $\epsilon(M) \geq 16$. Then, without loss of generality, $\left|X_{1}\right| \geq 8$. Pick $x \in X_{1}-L$. Then, as $\left|E\left(M_{2}\right) \cap L\right|=3$, the element $x$ can be on at most three lines. However we place the remaining five points, we will always get one of these lines having at least four points, which can be projected onto $L$, implying that $\left|E\left(M_{j}\right) \cap L\right| \geq 4$, a contradiction.

Therefore $\left|\left(X_{1} \cup X_{2}\right) \cap L\right| \neq 2$.

Hence, by Sublemmas 3.2.4.4 - 3.2.4.8, there are no possible values for $\left|\left(X_{1} \cup X_{2}\right) \cap L\right|$, so our original assumption, that $M$ has an exact vertical 3 -separation, is incorrect. So $M$ is vertically 4 -connected. 
Be regular and orderly in your life, so that you may be violent and original in your work.

Gustave Flaubert

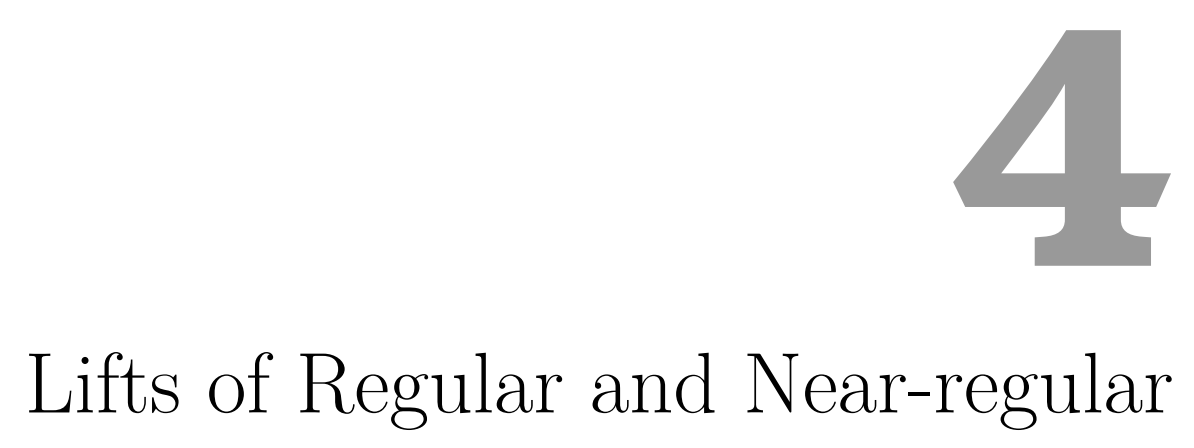

\subsection{Preliminaries}

Let $\mathcal{M}_{1}$ be the set of golden-mean matroids $M$ with the property that $M / e$ is regular for some $e \in E(M)$. Let $\mathcal{R}$ be the set of all minors of matroids in $\mathcal{M}_{1}$. Note that $M$ is in $\mathcal{R}$ if and only if $M$ is golden-mean, and there is some golden-mean single-element extension $N$ of $M$, by the element $e$, such that $N / e$ is regular. Equivalently, we might say that $M$ is a golden-mean lift of a regular matroid.

Now let $\mathcal{M}_{2}$ be the set of golden-mean matroids $M$ with the property that $M / e$ is near-regular for some $e \in E(M)$. Let $\mathcal{N}$ be the set of all minors of matroids in $\mathcal{M}_{2}$. As above, note that $M$ is in $\mathcal{N}$ if and only if $M$ is goldenmean, and there is some golden-mean single-element extension $N$ of $M$, by the element $e$, such that $N / e$ is near-regular. Equivalently, we might say that $M$ is a golden-mean lift of a near-regular matroid. Also note that $\mathcal{R}$ is a subclass of $\mathcal{N}$.

In this chapter, we prove that Conjecture 1.2.3 holds in the classes $\mathcal{R}$ and $\mathcal{N}$. 
Theorem 4.1.1. Let $\mathcal{R}$ be the class of matroids defined above. Then

$$
h_{\mathcal{R}}(r)=\left(\begin{array}{c}
r+3 \\
2
\end{array}\right)-5 \text {. }
$$

Furthermore, $M \in \mathcal{R}$ is maximum-sized if and only if $M \cong T_{r(M)}^{2}$.

Theorem 4.1.2. Let $\mathcal{N}$ be the class of matroids defined above. Then

$$
h_{\mathcal{N}}(r)=\left(\begin{array}{c}
r+3 \\
2
\end{array}\right)-5
$$

Furthermore, $M \in \mathcal{N}$ is maximum-sized if and only if $M$ is isomorphic to a member of $\mathscr{G}_{r(M)}$ when $r(M) \neq 3$, or a member of $\mathscr{G}_{3} \cup\{B R \backslash p\}$ when $r(M)=3$.

\subsubsection{Spikes}

For various results, we need to understand the golden-mean spikes.

Definition 4.1.3 (Ding et al. [4]). For $n \geq 3$, a simple matroid $M$ is a $\boldsymbol{n}$ spike with tip $\boldsymbol{t}$ if it satisfies the following properties.

(i) the ground set is the union of $n$ lines, known as legs, $L_{1}, \ldots, L_{n}$, all having three points and passing through a common point $t$;

(ii) for all $k$ in $\{1,2, \ldots, n-1\}$, the union of any $k$ of $L_{1}, \ldots, L_{n}$ has rank $k+1 ;$ and

(iii) $r\left(L_{1} \cup \cdots \cup L_{n}\right)=n$.

We will refer to an $n$-spike with tip $t$ as a $\boldsymbol{n}$-spike.

Let $S$ be a $n$-spike with tip $t$ representable over a field $\mathbb{F}$. If we choose a basis $\{1, \ldots, n\}$ containing exactly one element from each of the lines $L_{i}$, then $S$ can be represented in the form 


$$
\left[\begin{array}{ccccc|cccccc}
1 & 2 & 3 & \cdots & n & t & & & & & \\
1 & 0 & 0 & \cdots & 0 & 1 & x_{1} & 1 & 1 & \cdots & 1 \\
0 & 1 & 0 & \cdots & 0 & 1 & 1 & x_{2} & 1 & \cdots & 1 \\
0 & 0 & 1 & \cdots & 0 & 1 & 1 & 1 & x_{3} & \cdots & 1 \\
\vdots & \vdots & \vdots & \ddots & \vdots & \vdots & \vdots & \vdots & \vdots & \ddots & \vdots \\
0 & 0 & 0 & \cdots & 1 & 1 & 1 & 1 & 1 & \cdots & x_{n}
\end{array}\right]
$$

where $x_{1}, \ldots, x_{n}$ are elements of $\mathbb{F} \backslash\{1\}(\mathrm{Wu}[25])$.

We wish to characterise the golden-mean spikes. In particular, we are interested in the 4-spikes and 5-spikes.

Lemma 4.1.4. There is only one golden-mean 4-spike.

Proof. We prove this by an exhaustive computer search, the code for which is in Appendix 4.A.1. Upon running this code, we discover that up to isomorphism there is only one golden-mean 4-spike. This spike is represented over $G F(4)$ by the following matrix.

$$
\left[\begin{array}{lllllllll}
1 & 0 & 0 & 0 & 1 & 0 & 1 & 1 & 1 \\
0 & 1 & 0 & 0 & 1 & 1 & 0 & 1 & 1 \\
0 & 0 & 1 & 0 & 1 & 1 & 1 & \alpha & 1 \\
0 & 0 & 0 & 1 & 1 & 1 & 1 & 1 & \alpha
\end{array}\right]
$$

Lemma 4.1.5. There are no golden-mean 5-spikes.

Proof. We prove this by an exhaustive computer search, the code for which is in Appendix 4.A.2. Upon running this code, we discover that there are no golden-mean 5 -spikes. This has also been independently verified by Van Zwam (personal correspondence).

We want to know that it is not possible to extend a leg of the golden-mean 4-spike.

Lemma 4.1.6. If $M$ is a golden-mean 4-spike with tip $t, M^{\prime} \backslash e=M$, where $M^{\prime}$ is golden-mean, and $\{e, a, b\}$ is a triangle, where $\{t, a, b\}$ is a leg of $M$, then $\{t, e\}$ is a circuit. 
Proof. Assume for contradiction that $\{e, t\}$ is not a circuit, so $\{e, t, a, b\}$ is a $U_{2,4}$-restriction. Let $M$ be the matroid represented over $G F(4)$ by the following matrix.

$$
\left[\begin{array}{lllll}
t & 0 & 1 & 1 & 1 \\
1 & 1 & 0 & 1 & 1 \\
1 & 1 & 1 & \alpha & 1 \\
1 & 1 & 1 & 1 & \alpha
\end{array}\right]
$$

As $M$ is a spike, $\{t, a, b\}$ is a triangle. Hence, in $M^{\prime}$, where $\{e, a, b\}$ is a triangle, $\{t, e, a, b\}$ must be dependent. Now consider $M^{\prime} \backslash a=M^{\prime \prime}$. Note that $\{t, e, b\}$ is a triangle of $M^{\prime \prime}$ and that $M^{\prime \prime}$ is a 4-spike. By Lemma 4.1.4 there is only one golden-mean 4-spike, and so by Lemma 2.1.12 the column representing $e$ is parallel to the column representing $a$. Hence $\{e, a\}$ is a circuit, a contradiction.

Therefore $\{t, e\}$ is a circuit.

We rewrite Lemma 4.1.6 as the following Corollary.

Corollary 4.1.7. If $M$ is a simple golden-mean matroid, and $M^{\prime}$ is a 4-spike restriction of $M$ with $\ell$ as a leg of $M^{\prime}$, then $\ell$ is a flat in $M$.

\subsubsection{Small Circuits}

The family of matroids $T_{r}^{1}$ are the near-regular matroids represented over $\mathbb{U}_{1}$ by the following families of matrices. A geometric representation of $T_{3}^{1}$ is given in Figure 4.1.

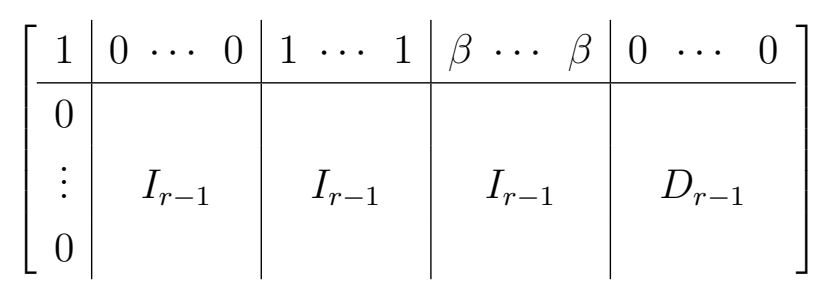

We also need to understand the simple regular and near-regular matroids with no circuits of size greater than four.

To that end, we need the following result. 


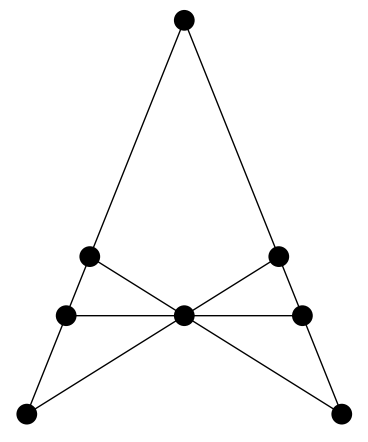

Figure 4.1: $T_{3}^{1}$

Lemma 4.1.8 (Whittle [22, Section 5]). Let $M$ be a simple near-regular matroid of rank three. Then $M$ is a restriction of $T_{3}^{1}$ (Figure 4.1).

Let $K_{2, r}^{\natural}$ be the family of near-regular matroids represented by the following family of matrices.

\begin{tabular}{|c|c|c|c|}
\hline 1 & $0 \cdots$ & $1 \cdots 1$ & $\beta \ldots \beta$ \\
\hline 0 & & & \\
\hline & $I_{r-1}$ & $I_{r-1}$ & $I_{r-1}$ \\
\hline
\end{tabular}

Note that $K_{2, r}^{\natural}$ consists of $r-1$ copies of $U_{2,4}$, known as legs, parallelconnected together at a point, known as the tip.

Lemma 4.1.9. If $M$ is a simple connected near-regular matroid with no circuit of size greater than four, then $M$ is a restriction of either $T_{3}^{1}$ or $K_{2, r}^{\natural}$, for some $r$.

Proof. We prove this by induction on $\epsilon(M)$.

If $M$ has rank at most three then the result follows from Lemma 4.1.8. Hence, we can assume that the rank of $M$ is at least four.

Firstly, assume that $M$ is not 3-connected. Then, by Theorem 8.3.1 of Oxley [10], $M=M_{X} \oplus_{2} M_{Y}$, for some matroids $M_{X}$ and $M_{Y}$. Note that Theorem 8.3.1 of Oxley [10] also implies that $\epsilon\left(M_{A}\right)<\epsilon(M)$, for $A \in\{X, Y\}$.

Proposition 7.1 .22 (ii) of Oxley [10] implies that both $M_{X}$ and $M_{Y}$ are connected. This means that we can apply the induction hypothesis to both $\operatorname{si}\left(M_{X}\right)$ and $\operatorname{si}\left(M_{Y}\right)$. 
Now assume that $b$, the basepoint of the 2-sum $M_{X} \oplus_{2} M_{Y}$, is in a circuit $C_{1}$ of size four in $M_{X}$. In $M_{Y}$, the element $b$ is in a circuit $C_{2}$ with $\left|C_{2}\right| \geq 3$, since $r\left(M_{Y}\right) \geq 2$ (as otherwise $M=M_{X} \oplus_{2} M_{Y}$ is just $M_{X}$ with a parallel class). Now, by Proposition 7.1.20 of Oxley [10], $\left(C_{1}-b\right) \cup\left(C_{2}-b\right)$ is a circuit of $M$ with at least five elements, a contradiction. Hence $b$ is not in any circuits of size four, and so $M_{X}$ is isomorphic to a restriction of $K_{2, r}^{\natural}$, where $b$ is either the tip or parallel to the tip. A symmetric argument shows that $M_{Y}$ is isomorphic to a restriction of $K_{2, r}^{\natural}$. If we 2-sum these matroids at the tip, we merely get a restriction of a larger $K_{2, r}^{\natural}$.

Now assume that $M$ is 3-connected. Then $M \backslash e$ is connected for any element $e \in E(M)$ by Proposition 8.2.8 of Oxley [10], and hence, by induction, it must be isomorphic to a restriction of $K_{2, r}^{\natural}$ for some $r$.

Consider $M \backslash e$. When we extend by e, $M$ must be 3 -connected, so we cannot add $e$ parallel to the tip, as $K_{2, r}^{\natural}$ is not 3-connected. If $e$ is in a triangle in $M$, then it is in the span of either one or two legs. As $M$ has rank at least four, it has at least three legs, so there is a leg $L$ of $M$ such that $L$ minus the tip is either a cocircuit of size at most two in $M$ or a cocircuit-circuit of size three, both of which contradict 3-connectivity. Since we chose $e$ arbitrarily, $M$ has no triangles. Hence for all $e \in E(M)$, the matroid $M \backslash e \cong M\left(K_{2, r}\right)$ for some $r$.

Let $\{x, y\}$ be a leg of $M \backslash e$. Then $\{e, x, y\}$ must be a cocircuit in $M$, as $\{x, y\}$ is a series pair. Hence $\{e, y\}$ must be a leg of $M \backslash x$. Let $\{u, v\}$ be some other leg of $M \backslash x$. Hence $\{e, u, v, y\}$ is a 4 -circuit, so it meets every leg in $M$, as a leg with $e$ added is a triad. This implies that there can be no more than three legs, so $r(M) \leq 4$, meaning that $r(M)=4$ and hence $\epsilon(M)=7$.

Now we see that $r\left(M^{*}\right)=3$, and hence we can apply Lemma 4.1.8 to see that $M$ is isomorphic to either $P_{7}^{*}$ or $O_{7}^{*}$, as these are the only 3 -connected seven element restrictions of $\left(T_{3}^{1}\right)^{*}$. Hence $M$ has circuits of size greater than four, a contradiction.

When $b \geq 1$, the graph $K_{2, b}^{+}$is the complete bipartite graph $K_{2, b}$ with the addition of an edge joining the partition of size two. A drawing of $K_{2,5}^{+}$is given in Figure 4.2.

We now define some terminology related to $M\left(K_{2, b}^{+}\right)$. We will explore $M\left(K_{2, b}^{+}\right)$ 


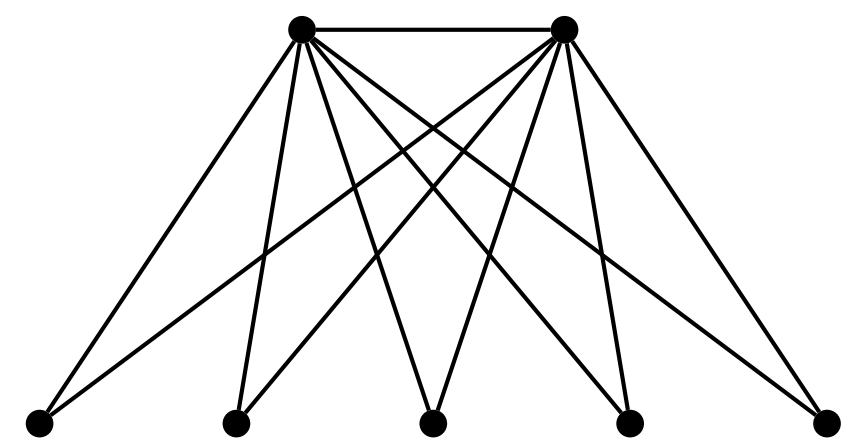

Figure $4.2: K_{2,5}^{+}$

more in Section 4.3.2.

Definition 4.1.10. Each three-point line in $M\left(K_{2, b}^{+}\right)$is known as a $\mathbf{l i m b}$. If $b=1$, then the head of $M\left(K_{2, b}^{+}\right)$is any element of $M\left(K_{2, b}^{+}\right)$. If $b>1$, then the head of $M\left(K_{2, b}^{+}\right)$is the element of $M\left(K_{2, b}^{+}\right)$that is in $b$ three-point lines.

By considering the regular restrictions of $T_{3}^{1}$ and $K_{2, r}^{\natural}$ we get the following corollary.

Corollary 4.1.11. If $M$ is a connected regular matroid and $M$ has no circuit of size greater than four, then either $\operatorname{si}(M) \cong M\left(K_{4}\right)$, or $\operatorname{si}(M) \cong M\left(K_{2, b}\right)$ for some $b$, or $\operatorname{si}(M) \cong M\left(K_{2, b}^{+}\right)$for some $b$.

\subsection{3 $\mathcal{L}(M, e)$}

We often consider the matroid obtained by restricting to the long lines through $e$, contracting $e$ and then simplifying. In this section, we develop this idea.

Definition 4.1.12. Let $M$ be a matroid, and let $e$ be an element of $M$, and let $\mathbb{Q}$ be the set of long lines of $M$. Let $X=\{e\} \cup\{f \in E(M) \mid \exists L \in$ $\mathfrak{Q}$ with $e, f \in L\}$. Then $\mathcal{L}(M, e)$ is defined to be $\operatorname{si}((M \mid X) / e)$.

Note that $\mathcal{L}(M, e)$ is isomorphic to a restriction of $M / e$. We wish to restrict the circuits contained in $\mathcal{L}(M, e)$.

Lemma 4.1.13. Let $M=(E, r)$ be a maximum-sized golden-mean matroid, and let $e$ be an element of $M$. If $\mathcal{L}(M, e)$ contains two circuits $C_{1}$ and $C_{2}$, such that $\left|C_{1}\right|=\left|C_{2}\right|=4$, then $\left|C_{1} \cap C_{2}\right| \neq 3$. 
Proof. Assume that $\left|C_{1} \cap C_{2}\right|=3$. Then $C_{1}=\left\{c_{1}, x, y, z\right\}$ and $C_{2}=$ $\left\{c_{2}, x, y, z\right\}$. As $C_{i}$ for $i \in\{1,2\}$ is from $\mathcal{L}(M, e)$, each element of $C_{i}$ corresponds to a long line in $M$. Let these lines be $l_{a}^{\prime}$ for $a \in C_{1} \cup C_{2}$. For each $l_{a}^{\prime}$, let $l_{a}$ be a three-point restriction of $l_{a}^{\prime}$ such that $e \in l_{a}$. Let

$$
S_{i}=\bigcup_{a \in C_{i}} l_{a}
$$

for $i \in\{1,2\}$. We claim that $S_{1}$ and $S_{2}$ are 4 -spikes.

To see this, note that $r\left(S_{i}\right)=4$, and for all $k \in\{1,2,3\}$ the union of any $k$ long lines from $S_{i}$ has rank $k+1$.

Let $N=M \mid\left(S_{1} \cup S_{2}\right)$. So $N$ consists of five long lines, in particular the long lines $\left\{e, c_{1}, c_{1}^{\prime}\right\}$ and $\left\{e, c_{2}, c_{2}^{\prime}\right\}$ are in $N$. Note that $N \backslash c_{1} \backslash c_{1}^{\prime}=S_{2}$ and $N \backslash c_{2} \backslash c_{2}^{\prime}=S_{1}$.

So $N \backslash c_{2} \backslash c_{2}^{\prime}$ has the $G F(4)$ representation

$$
U=\left[\begin{array}{lllllllll}
1 & 0 & 0 & 0 & 1 & a & 1 & 1 & 1 \\
0 & 1 & 0 & 0 & 1 & 1 & b & 1 & 1 \\
0 & 0 & 1 & 0 & 1 & 1 & 1 & c & 1 \\
0 & 0 & 0 & 1 & 1 & 1 & 1 & 1 & d
\end{array}\right]
$$

Furthermore, if we delete $c_{1}$ and $c_{1}^{\prime}$ instead, we get

$$
U^{\prime}=\left[\begin{array}{lllllllll}
1 & 0 & 0 & 0 & 1 & a & 1 & 1 & 1 \\
0 & 1 & 0 & 0 & 1 & 1 & b & 1 & 1 \\
0 & 0 & 1 & 0 & 1 & 1 & 1 & c & 1 \\
0 & 0 & 0 & 1 & 1 & 1 & 1 & 1 & d^{\prime}
\end{array}\right]
$$

It follows from Lemma 4.1.4 that $(a, b, c, d)$ and $\left(a, b, c, d^{\prime}\right)$ are equal up to permutation. Hence $d=d^{\prime}$.

As we have uniqueness of representations by Lemma 2.1.12, we can combine $U$ and $U^{\prime}$ in the obvious way to see that $c_{1}$ and $c_{2}$ are parallel in $N$. This is a contradiction, so $N$ cannot exist, and hence $\left|C_{1} \cap C_{2}\right| \neq 3$.

Lemma 4.1.14. Let $M$ be a maximum-sized golden-mean matroid, and let 
e be a point of $M$. Then $\mathcal{L}(M, e)$ is near-regular.

Proof. If $\mathcal{L}(M, e)$ is not near-regular, then it must contain an excluded minor for the class of near-regular matroids, which are listed in [5, Theorem 1.2]. As $\mathcal{L}(M, e)$ is a minor of a golden-mean matroid, the excluded minor must be golden-mean. By considering the ten excluded minors for near-regular matroids, we see that only $U_{2,5}$ and $U_{3,5}$ are golden-mean.

If $\mathcal{L}(M, e)$ has a $U_{2,5}$-minor, then we can find a rank-three restriction of $M$ with eleven points and five copunctual lines, a contradiction to Lemma 2.3.1. If $\mathcal{L}(M, e)$ has a $U_{3,5}$-minor, then we can find two 4 -circuits in $U_{3,5}$ that share three elements. This is a contradiction by Lemma 4.1.13, so $\mathcal{L}(M, e)$ can not have a $U_{3,5}$-minor, and hence $\mathcal{L}(M, e)$ is near-regular.

We want to know exactly what $\mathcal{L}(M, e)$ consists of. The next two results provide this.

Lemma 4.1.15. $\mathcal{L}(M, e)$ has no circuits of size five or greater.

Proof. Assume that $\mathcal{L}(M, e)$ has a circuit $C=\left\{c_{1}, \ldots, c_{5}\right\}$ of size five. By the definition of $\mathcal{L}(M, e)$, each $c_{i}$ is in a parallel class of size at least two in $M / e$. Let $d_{i} \neq c_{i}$ be an element from the parallel class containing $c_{i}$, for $i \in 1, \ldots, 5$. We claim that $S=M \mid\left\{e, c_{1}, \ldots, c_{5}, d_{1}, \ldots, d_{5}\right\}$ is a 5 -spike.

To see this, note that $r(S)=5$, and that for all $i$, the three elements $\left\{e, c_{i}, d_{i}\right\}$ form a line $L_{i}$. Also, for all $k$ in $\{1,2,3,4\}$, the union of any $k$ of $L_{1}, \ldots, L_{5}$ has rank $k+1$. Hence $S$ is a 5 -spike.

However, there are no golden-mean 5 -spikes by Lemma 4.1.5, and so this is a contradiction. Hence $\mathcal{L}(M, e)$ can have no circuits of size five or greater.

Corollary 4.1.16. Let $M$ be a maximum-sized golden mean matroid, and let $e$ be a point of $M$. Then the connected components of $\mathcal{L}(M, e)$ are restrictions of either $T_{3}^{1}$ or $K_{2, x}^{\natural}$, for some $x$.

Proof. From Lemmas 4.1.14 and 4.1.15, we know that $\mathcal{L}(M, e)$ is a nearregular matroid with no circuits of size five or greater. The result now follows from Lemma 4.1.9.

Corollary 4.1.17. $\mathcal{L}(M, e)$ cannot have $P_{5}$ (Figure 4.3) as a minor. 


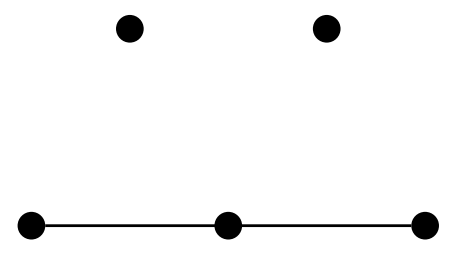

Figure 4.3: $P_{5}$

Proof. This follows from Lemma 4.1.13.

By considering the restrictions of $T_{3}^{1}$ and $K_{2, r}^{\natural}$ that do not have a $P_{5}$-minor, we get the following result.

Corollary 4.1.18. Let $M$ be a maximum-sized golden mean matroid, and let $e$ be a point of $M$. Then the connected components of $\mathcal{L}(M, e)$ are isomorphic to one of the following matroids:

(i) $U_{1,1}$,

(ii) $U_{2,4}$,

(iii) $M\left(K_{4}\right)$,

(iv) $M\left(K_{2, b}\right)$ for some $b \geq 2$, or

(v) $M\left(K_{2, b}^{+}\right)$for some $b \geq 1$.

\subsubsection{Deficit Functions}

As $\mathcal{L}(M, e)$ is the simplification of a contraction of a collection of long lines, we lose the length of each line upon this contraction. To regather this information into a useable format, we define the weight functions.

Henceforth, we are going to let $M$ be a counterexample to either Theorem 4.1.1 or Theorem 4.1.2.

Function 4.1.19. The first function we are defining is the weight function $w$.

Let $e$ be an element of the matroid $M$. If $i \in E(M) \backslash e$, then

$$
w_{i}=\left|\mathrm{cl}_{M}(\{e, i\})\right|
$$


If $N$ is a component of $\mathcal{L}(M, e)$ (denoted by $N \lessdot \mathcal{L}(M, e))$, then

$$
w(N)=\sum_{i \in E(N)}\left(w_{i}-2\right)
$$

By the definition of $\mathcal{L}(M, e)$, the minimum value of $w_{i}$ is three. Also, as $M$ is $G F(4)$-representable, the maximum value is five. Hence for all $i \in$ $E(\mathcal{L}(M, e))$,

$$
3 \leq w_{i} \leq 5
$$

Let $M$ have rank $r$. Then

$$
\sum_{N \ll \mathcal{L}(M, e)} r(N)=r(\mathcal{L}(M, e)) \leq r-1 .
$$

We consider two possible options for $M / e$, and construct a function for each.

First, consider the case when $M / e$ is regular. In this case, $\epsilon(M / e) \leq\left(\begin{array}{l}r \\ 2\end{array}\right)$ by Theorem 1.1.9. Also, as $M$ is a counterexample to Theorem 4.1.1, $\epsilon(M) \geq$ $\left(\begin{array}{c}r+3 \\ 2\end{array}\right)-5$.

Therefore

$$
\epsilon(M)-\epsilon(M / e) \geq\left(\begin{array}{c}
r+3 \\
2
\end{array}\right)-5-\left(\begin{array}{l}
r \\
2
\end{array}\right)=3 r-2 .
$$

Hence

$$
\sum_{N \lessdot \mathcal{L}(M, e)} w(N)+1 \geq 3 r-2
$$

Combining (4.1.2) with (4.1.3), we obtain

$$
3 \sum_{N \lessdot \mathcal{L}(M, e)} r(N) \leq \sum_{N \lessdot \mathcal{L}(M, e)} w(N) .
$$

This motivates the second function.

Function 4.1.20. The next function we define is the regular deficit function $d_{r}$. If $N \lessdot \mathcal{L}(M, e)$, then

$$
d_{r}(N)=3 r(N)-w(N) .
$$


Rewriting (4.1.4) using the regular deficit function, we get

$$
\sum_{N \lessdot \mathcal{L}(M, e)} d_{r}(N) \leq 0
$$

Now consider the case when $M / e$ is near-regular. In this case, $\epsilon(M / e) \leq$ $\left(\begin{array}{c}r+1 \\ 2\end{array}\right)-2$ by Theorem 1.1.12. Also, as $M$ is a counterexample to Theorem 4.1.2, $\epsilon(M) \geq\left(\begin{array}{c}r+3 \\ 2\end{array}\right)-5$.

Therefore

$$
\epsilon(M)-\epsilon(M / e) \geq\left(\begin{array}{c}
r+3 \\
2
\end{array}\right)-5-\left(\begin{array}{c}
r+1 \\
2
\end{array}\right)+2=2 r .
$$

Hence

$$
\sum_{N \lessdot \mathcal{L}(M, e)} w(N)+1 \geq 2 r
$$

Combining (4.1.2) with (4.1.7), we obtain

$$
2 \sum_{N \lessdot \mathcal{L}(M, e)} r(N)+1 \leq \sum_{N \lessdot \mathcal{L}(M, e)} w(N) .
$$

This motivates the third function.

Function 4.1.21. The next function we define is the near-regular deficit function $d_{n}$. If $N \lessdot \mathcal{L}(M, e)$, then

$$
d_{n}(N)=2 r(N)-w(N)
$$

Rewriting (4.1.8) using the near-regular deficit function, we get

$$
\sum_{N \lessdot \mathcal{L}(M, e)} d_{n}(N) \leq-1
$$

We now go through each possible component of $\mathcal{L}(M, e)$ in turn and calculate the minimum value of both deficit functions. We do this by computing the maximum weight and then applying Functions 4.1.20 and 4.1.21.

Lemma 4.1.22. Let $N$ be a component of $\mathcal{L}(M, e)$ such that $N \cong M\left(K_{2, b}\right)$ for some $b \geq 2$. Then $w_{i}=3$ for all $i \in E(N)$, and hence the maximum value of $w(N)$ is $2 b$. 
Proof. The matroid $M\left(K_{2, b}\right)$ consists of $b$ copies of $U_{2,3}$, known as legs, 2summed at a point, and hence has rank $b+1$. Each leg has two points on it, and any two legs, when considered independently, give a copy of $U_{3,4}$ in $\mathcal{L}(M, e)$, which leads to a 4 -spike in $M$. It follows from Corollary 4.1.7 that the maximum size of the closure of the leg of a golden-mean 4-spike is three. Therefore from Function 4.1.19 we get that the maximum weight of $M\left(K_{2, b}\right)$ is $2 b$.

Corollary 4.1.23. The minimum regular deficit of $M\left(K_{2, b}\right)$ for some $b \geq 2$ $i s b+3$.

Corollary 4.1.24. The minimum near-regular deficit of $M\left(K_{2, b}\right)$ for some $b \geq 2$ is two.

Lemma 4.1.25. Let $N$ be a component of $\mathcal{L}(M, e)$ such that $N \cong M\left(K_{4}\right)$. Then $w_{i}=3$ for all $i \in E(N)$, and hence the maximum value of $w(N)$ is six.

Proof. The matroid $M\left(K_{4}\right)$ has six points and rank three. Each point, when considered in the correct set of four points, gives a copy of $U_{3,4}$ in $\mathcal{L}(M, e)$, which leads to a 4-spike in $M$. It follows from Corollary 4.1.7 that the maximum size of the closure of the leg of a golden-mean 4-spike is three. Therefore from Function 4.1.19 we get that the maximum weight of $M\left(K_{4}\right)$ is six.

Corollary 4.1.26. The minimum regular deficit of $M\left(K_{4}\right)$ is three.

Corollary 4.1.27. The minimum near-regular deficit of $M\left(K_{4}\right)$ is zero.

Lemma 4.1.28. Let $N$ be a component of $\mathcal{L}(M, e)$ such that $N \cong M\left(K_{2, b}^{+}\right)$ for some $b \geq 2$. Then $w_{i}=3$ for all $i \in E(N) \backslash\{p\}$, where $p$ is the head of $N$, and hence the maximum value of $w(N)$ is $2 b+3$.

Proof. Recall that the matroid $M\left(K_{2, b}^{+}\right)$is the matroid $M\left(K_{2, b}\right)$ with the addition of the basepoint $p$, known as the head of $M\left(K_{2, b}^{+}\right)$, from the 2 -sum. There is no restriction on $p$ except for (4.1.1), and all other points have the same restrictions as in the proof of Lemma 4.1.22. Hence the maximum weight is the maximum weight of $M\left(K_{2, b}\right)$ plus the extra weight from $p$, giving $2 b+3$. 
Lemma 4.1.29. Let $N$ be a component of $\mathcal{L}(M, e)$ such that $N \cong M\left(K_{2,1}^{+}\right)$. Then

(i) if $w_{i}=5$ for some $i \in E(N)$, then $w_{j}=3$ for all $j \in E(N) \backslash\{i\}$, and

(ii) if $w_{i}=4$ for some $i \in E(N)$, then $w_{j}=3$ for some $j \in E(N) \backslash\{i\}$.

Hence the maximum value of $w(N)$ is five.

Proof. Note that $M\left(K_{2,1}^{+}\right) \cong U_{2,3}$. Let $E(N)=\{x, y, z\}$ such that $w_{x} \geq$ $w_{y} \geq w_{z}$.

First, assume that $w_{x}=5$ and $w_{y}>3$. Then, in $M$, there are there three coplanar lines containing $e$; one of length five, one of length at least four, and one of length at least three. However, this plane is not golden-mean by Lemma 2.3.1, and so $w_{y}=3$, and case (i) follows.

Now assume that $w_{z}=4$. If case (ii) fails, then, in $M$, there are three coplanar four-point lines containing $e$, meaning that $M$ has a ten-element rank-three restriction with three copunctual lines, each of which contains at least four elements. This cannot happen by Lemma 2.3.1, and hence $w_{z} \neq 4$, and case (ii) follows.

It both cases, we can easily verify that $w(N) \leq 5$.

Corollary 4.1.30. The minimum regular deficit of $M\left(K_{2, b}^{+}\right)$for some $b \geq 1$ is $b$.

Corollary 4.1.31. The minimum near-regular deficit of $M\left(K_{2, b}^{+}\right)$for some $b \geq 1$ is -1 .

Lemma 4.1.32. Let $N$ be a component of $\mathcal{L}(M, e)$ such that $N \cong U_{2,4}$. Then $w_{i}=3$ for all $i \in E(N)$, and hence the maximum value of $w(N)$ is four.

Proof. Assume that one point of $U_{2,4}$ corresponds to a line of length four. Then, in $M$, we have a ten-element rank-three restriction of $M$ with all elements being on four lines, each of which contains $e$, leading to a contradiction to Lemma 2.3.1. Hence the maximum weight of $U_{2,4}$ is four.

Corollary 4.1.33. The minimum near-regular deficit of $U_{2,4}$ is zero. 
The only component of $\mathcal{L}(M, e)$ we have yet to consider is $U_{1,1}$. The only restriction on the element $u$ of $U_{1,1}$ is (4.1.1). Hence $w_{u} \leq 5$, and so the maximum weight of $U_{1,1}$ is three.

Corollary 4.1.34. The minimum regular deficit of $U_{1,1}$ is zero.

Corollary 4.1.35. The minimum near-regular deficit of $U_{1,1}$ is -1 .

\subsection{Lifts of Regular}

We first prove Theorem 4.1.1. To do this, we consider a counterexample $M$ of minimum rank to Theorem 4.1.1 and show that $M$ does not exist. We are able to assume that $M$ is maximum-sized. Note that as Lemma 2.3.1 characterises all the golden-mean matroids of rank three, we are able to assume that $r(M) \geq 4$.

First, we need to find an element $e$ of $M$ such that $M / e$ is regular.

Lemma 4.2.1. Let $M$ be a maximum-sized member of $\mathcal{R}$. Then there exists $e \in E(M)$ such that $M / e$ is regular.

Proof. Assume for a counterexample that no such $e$ exists. Then $M$ has a single element extension $N$ by $f$, where $N$ is golden-mean and $N / f$ is regular. However, $M$ is maximum-sized, so $f$ must be parallel to an element $e$ of $M$. But then $M / e$ is regular, a contradiction.

Hence such an e must exist.

We now characterise $\mathcal{L}(M, e)$.

Lemma 4.2.2. Let $M$ be a counterexample to Theorem 4.1 .1 of minimum rank, and assume that $M$ is maximum-sized. Let $e \in E(M)$ be such that $M / e$ is regular. Then $\mathcal{L}(M, e)$ is a collection of coloops.

Proof. As $\mathcal{L}(M, e)$ is a restriction of $M / e$, it follows that $\mathcal{L}(M, e)$ is regular. Hence we can apply Function 4.1.20 to get (4.1.5), which tells us that the sum of the regular deficits of all components of $\mathcal{L}(M, e)$ must be non-positive. From Corollaries 4.1.23, 4.1.26, 4.1.30, and 4.1.34, it is easy to see that the only way to make (4.1.5) true is if every component of $\mathcal{L}(M, e)$ is isomorphic to $U_{1,1}$. 
Corollary 4.2.3. Let $M$ be a rank-r counterexample to Theorem 4.1 .1 of minimum rank, and assume that $M$ is maximum-sized. Let $e \in E(M)$ be such that $M / e$ is regular. Then there are exactly $r-1$ long lines through $e$, all of length five.

Proof. If there are $r$ long lines through $e$, then, as each long line corresponds to a coloop in $\mathcal{L}(M, e)$, the rank of $\mathcal{L}(M, e)$ must be at least $r$, and therefore $r(M / e) \geq r$, which is a contradiction.

Hence there can be at most $r-1$ long lines containing $e$, and since $w(N) \leq 3$ for each $N \lessdot \mathcal{L}(M, e)$, it follows that

$$
\sum_{N \lessdot \mathcal{L}(M, e)} w(N)+1 \leq 3 r-2 .
$$

When we combine (4.1.3) with (4.2.1), we see that

$$
\sum_{N \lessdot \mathcal{L}(M, e)} w(N)+1=3 r-2 .
$$

The only way to make (4.2.2) true is to have $r(\mathcal{L}(M, e))=r-1$ and $w(N)=3$ for all $N$. Therefore there are $r-1$ long lines containing $e$, all of length five.

Lemma 4.2.4. Let $M$ be a rank-r counterexample to Theorem 4.1 .1 of minimum rank, and assume that $M$ is maximum-sized. Let $e \in E(M)$ be such that $M /$ e is regular. Then $\operatorname{si}(M / e) \cong M\left(K_{r}\right)$.

Proof. As $M$ is a counterexample to Theorem 4.1.1,

$$
\epsilon(M) \geq\left(\begin{array}{c}
r+3 \\
2
\end{array}\right)-5 .
$$

It follows from Corollary 4.2.3 that

$$
\epsilon(M)-\epsilon(M / e)=3 r-2 .
$$

So

$$
\epsilon(M / e) \geq\left(\begin{array}{c}
r+3 \\
2
\end{array}\right)-5-3 r+2=\left(\begin{array}{l}
r \\
2
\end{array}\right)
$$




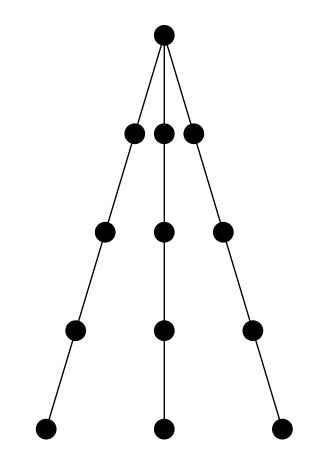

Figure 4.4: Forbidden Configuration from Lemma 4.2.5

As $M / e$ is regular of rank at most $r-1$, it follows from Theorem 1.1.9 that

$$
\epsilon(M / e) \leq\left(\begin{array}{l}
r \\
2
\end{array}\right)
$$

Hence

$$
\epsilon(M / e)=\left(\begin{array}{l}
r \\
2
\end{array}\right)
$$

and it follows from Theorem 1.1.9 that $\operatorname{si}(M / e) \cong M\left(K_{r}\right)$.

Lemma 4.2.5. Let $M$ be a rank-r counterexample to Theorem 4.1.1 of minimum rank, and assume that $M$ is maximum-sized. Let $e \in E(M)$ be such that $M / e$ is regular. Then any two elements in $\mathcal{L}(M, e)$ will be on a triangle in $\operatorname{si}(M / e)$.

Proof. Firstly, note that it follows from Lemma 4.2.2 that the elements of $\mathcal{L}(M, e)$ form a basis of $\operatorname{si}(M / e)$. Now let $x$ be an element from $\operatorname{si}(M / e)$ that is not in $\mathcal{L}(M, e)$. Assume that $x$ is not in a line of $\operatorname{si}(M / e)$ with two elements from $\mathcal{L}(M, e)$. If $L_{i}$ and $L_{j}$ are any long lines containing $e$, then $r\left(L_{i} \cup L_{j}\right)=3$, and $r\left(L_{i} \cup L_{j} \cup x\right)=4$. Hence $r_{M / x}\left(L_{i} \cup L_{j}\right)=3$, so $L_{1}, \ldots, L_{r-1}$ are distinct lines of $M / x$. Hence $\mathcal{L}(M / x, e)$ has $r-1$ points and rank at most $r-2$, so $\mathcal{L}(M / x, e)$ contains a circuit, $C$.

If $|C|=3$, then there exist three copunctal five-point lines in rank-three (Figure 4.4) in $M / x$, a contradiction to Lemma 2.3.1. If $|C|=4$, then using the same technique as in Lemma 4.1 .15 a 4 -spike restriction of $M / x$ can be found. However, as $e$ is on five-point lines, the closures of the legs of this spike have cardinality five, leading to a contradiction to Corollary 4.1.7. Finally, 
if $|C| \geq 5$ then a 5 -spike can be found, contradicting Lemma 4.1.5. Hence $C$ does not exist, so $x$ must be on a triangle with two elements from $\mathcal{L}(M, e)$. There are $\left(\begin{array}{c}r-1 \\ 2\end{array}\right)$ pairs of elements from $\mathcal{L}(M, e)$, and each of the $\left(\begin{array}{l}r \\ 2\end{array}\right)-(r-1)=$ $\left(\begin{array}{c}r-1 \\ 2\end{array}\right)$ elements from $\operatorname{si}(M / e) \backslash \mathcal{L}(M, e)$ must be on a line with two elements from $\mathcal{L}(M, e)$. As $\operatorname{si}(M / e)$ is binary, we have that every pair of elements from $\mathcal{L}(M, e)$ is on a triangle in $\operatorname{si}(M / e)$.

Now we can prove Theorem 4.1.1. Let $M$ be a rank-r counterexample to Theorem 4.1.1 of minimum rank. Wa can assume that $M$ is maximum-sized. By Lemma 4.2.1, we can find $e \in E(M)$ such that $M / e$ is regular.

We are going to construct a $G F(4)$-representation for $M$. By Corollary 4.2.3, there are exactly $r-1$ long lines passing through $e$, each of length five. Lemma 4.2.4 tells us that $\operatorname{si}(M / e) \cong M\left(K_{r}\right)$.

Let $L_{i}$ and $L_{j}$ be lines passing through $e$. Then there is a unique element $f \in \operatorname{si}(M / e)$ that is on the line in $\operatorname{si}(M / e)$ between the point corresponding to $L_{i}-e$ and the point corresponding to $L_{j}-e$. Let us consider $M$ restricted to the union of $L_{i}, L_{j}$, and $f$. This has rank three, and contains two lines of length five. When we contract $f$, we must get a copy of $U_{2,5}$ since $M$ is representable over $G F(4)$. This means that $f$ is on four different triangles. So the rank-three restriction is just a copy of $T_{3}^{2}$, as in Figure 2.2.

Now pick an arbitrary element $x_{i} \in L_{i}$. For every other line $L_{j}$ consider the element $f_{i, j}$ that is on the line between $L_{i}-e$ and $L_{j}-e$ in $\operatorname{si}(M / e)$. Let $x_{j}$ be the element of $L_{j}$ that is contained in a triangle with $x_{i}$ and $f_{i, j}$.

Assume that $M$ is represented over $G F(4)$ by $\left[I_{r} \mid A\right]$, where the first column is labelled by $e$. Since $\operatorname{si}(M / e)$ is isomorphic to $M\left(K_{r}\right)$ by Lemma 4.2.4, the uniqueness of representation of binary matroids over any field ([10, Proposition 6.6.5]) tells us that we can assume that

$$
\left[I_{r} \mid A\right]=\left[I_{r} \mid \begin{array}{c|c}
* & D^{\prime}
\end{array}\right]
$$

where the columns of $I_{r}$ are labelled by $\left(e, x_{1}, \ldots, x_{r-1}\right)$, and the columns of $A^{\prime}$ are the elements that are in five-point lines with $e$, but not in $\operatorname{si}(M / e)$.

Since each $x_{i}$ is in a five-point line with $e$, and $e$ is on no other long lines, we 
see that $M$ must be represented by the matrix

$$
\left[\begin{array}{c|c|c|c|c|c}
1 & 0 \cdots & 1 \cdots 1 & \alpha \cdots \alpha & \alpha^{2} \cdots \alpha^{2} & * \cdots * \\
\hline 0 & & & & & \\
\vdots & I_{r-1} & I_{r-1} & I_{r-1} & I_{r-1} & D_{r-1} \\
0 & & & & &
\end{array}\right]
$$

Each element in $\operatorname{si}(M / e) \backslash \mathcal{L}(M, e)$, and hence each element represented by a column of the $D_{r-1}$ block is in a triangle with two elements $x_{i}$ and $x_{j}$, for some $\{i, j\} \subseteq\{1, \ldots, r-1\}$, by Lemma 4.2.5. Therefore the row above the $D_{r-1}$ block must contain zeros, and so $M$ must be represented by the matrix

$$
\left[\begin{array}{c|c|c|c|c|c}
1 & 0 \cdots & 1 \cdots 1 & \alpha \cdots & \alpha^{2} \cdots \alpha^{2} & 0 \cdots 0 \\
\hline 0 & & & & & \\
\vdots & I_{r-1} & I_{r-1} & I_{r-1} & I_{r-1} & D_{r-1} \\
0 & & & & &
\end{array}\right]
$$

This matrix is identical to the $G F(4)$ matrix for $T_{r}^{2}$ given in Figure 2.5.1, and hence $M \cong T_{r}^{2}$. However, this means that $M$ is not a counterexample. Hence Theorem 4.1.1 is true.

From Theorem 4.1.1 we get the following two corollaries, which will be used in later work. The first corollary is easy, while the second needs a little work.

Corollary 4.2.6. Let $M$ be a counterexample to Conjecture 1.2.3. Then $M$ has no element e such that $M /$ e is regular.

Corollary 4.2.7. Let $M$ be a counterexample to Conjecture 1.2.3 with no BR-minor. Then $M$ has no copunctual five-point lines.

Proof. Assume that $M$ does have a point $e$ such that $e$ is on at least two fivepoint lines. By Corollary 4.2.6, $M / e$ is not regular. Hence $M / e$ is not binary, and therefore it has a $U_{2,4}$-minor. Let $x$ and $y$ be elements corresponding to two five-point lines in $\mathcal{L}(M, e)$. By Theorem 3.2.4, $M$ is vertically 4connected, so $\operatorname{si}(M / e)$ is 3 -connected by Lemma 3.2.3. Now we can apply Lemma 2.4.2 and find a $U_{2,4}$-minor using $x$ and $y$ in $M / e$. Hence when we de-contract $e$, in $M$, we can find a rank-three eleven-element restriction of $M$ containing two copunctual five-point lines, which is a contradiction to Lemma 2.3.1. 
Therefore there can be at most one five-point line going through $e$.

\subsection{Lifts of Near-regular}

We now prove Theorem 4.1.2 by showing that no counterexamples exist. We can assume that a counterexample is maximum-sized and of smallest possible rank. Note that as Lemma 2.3.1 characterises all the golden-mean matroids of rank-three, we are able to assume that $r(M) \geq 4$.

Throughout this section, we use the following results.

Lemma 4.3.1 (Bixby, Theorem 1 [2]). Let $M$ be a 3-connected matroid on $E$, and let $a \in E$. Then either $\operatorname{co}(M \backslash a)$ or $\operatorname{si}(M / a)$ is 3-connected.

Lemma 4.3.2. Let $M$ be a 3-connected matroid, and let $X$ be a non-empty subset of $E(M)$ such that $X$ is a union of long lines from $M$. Then $M$ has a 3-connected minor $M^{\prime}$ such that $M^{\prime}|X=M| X$ and $X$ spans $M^{\prime}$.

Proof. Assume that $M$ is a minimal counterexample to the lemma. If $X$ is the union of only one line, then $M^{\prime}=M \mid X$ is the desired minor. Therefore $X$ contains at least two lines. Let $F$ be the closure of $X$ in $M$. If $F=E(M)$ then we are done. Therefore we assume that there is a $x$ in $E(M) \backslash E(F)$. By Lemma 4.3.1, either $\operatorname{si}(M / x)$ or $\operatorname{co}(M \backslash x)$ is 3-connected. Assume that the former holds. Let $N=\operatorname{si}(M / x)$. We can assume that $X$ is a subset of $E(N)$. Now $N|X=M| X$, and the minimality of $M$ is contradicted. Therefore $\operatorname{co}(M \backslash x)$ is 3-connected.

Let $S$ contain all but one element from each non-trivial series class in $M \backslash x$. Thus $M \backslash x / S$ is isomorphic to $\operatorname{co}(M \backslash x)$. Any circuit contained in $S$ must intersect a series pair in a single element, which is impossible. Therefore $S$ is independent in $M \backslash x$. The same argument shows that $S$ is a flat, unless some series class of $M \backslash x$ is a circuit. But $M \backslash x$ is connected by [10, Proposition 8.2.8], so this would imply that $M \backslash x$ is a circuit and that $M$ is a corank-2 uniform matroid. In this case $M$ contains no lines, unless $M$ is isomorphic to $U_{2,4}$, but this contradicts the fact that $X$ does not span $M$. Therefore $S$ is an independent flat in $M \backslash x$.

We will show that we can assume that $S$ and $F$ have an element in common. Assume otherwise, so that $S \cap F=\varnothing$. If $r(S)+r(F)=r(S \cup F)$, then 
$(M \backslash x / S)|F=M| F$, and the minimality of $M$ is contradicted. Therefore there is some circuit $C$ contained in $S \cup F$ that contains elements from both $S$ and $F$. Let $S^{\prime}$ be a series class in $M \backslash x$ such that $S^{\prime}$ contains elements of $C$. Then $S^{\prime} \subseteq C$, for otherwise $C$ intersects a series pair in a single element. The single element $s$ in $S^{\prime} \backslash S$ must therefore belong to $F$. If $s$ is not in $X$, then there is a circuit $C^{\prime}$ contained in $X \cup\{s\}$ that contains $s$. In this case $C^{\prime}$ intersects a series pair in a single element. Therefore $s$ is in $X$. Now we can use the set $\left(S \backslash\left\{s^{\prime}\right\}\right) \cup\{s\}$ instead of $S$, where $s^{\prime}$ is an element of $S^{\prime} \backslash\{s\}$. Therefore we can assume that $s$ is an element in $S \cap X$.

Since every element in $X$ is in a long line in $M \backslash x$, we can find a long line of $M \backslash x$ that intersects a series class. In order to avoid having a circuit meet a series pair in a single element, there must be a series class of size two in $M \backslash x$ that spans a circuit of size three. This implies that $\operatorname{co}(M \backslash x)$ contains a parallel pair. Since $\operatorname{co}(M \backslash x)$ is 3 -connected, we see that $\operatorname{co}(M \backslash x)$ is a restriction of $U_{1,3}$.

Switching to the dual, $M^{*} / x$ is obtained from $U_{1,1}, U_{1,2}$, or $U_{2,3}$ by adding parallel elements. In the first and second cases $M^{*}$ is a rank-2 uniform matroid. This means $M$ must be isomorphic to $U_{2,4}$, and $X$ must be the entire ground set, a contradiction. Therefore $M^{*}$ consists of three lines in rank three, each of which contains $x$. Since $X$ contains at least two lines in $M$, it follows that $M^{*}$ contains at least two triads. This means that the lines containing $x$ can contain no more than three elements. In fact, exactly one of them contains only two elements, so $|E(M)|=6$. Thus $r(M)=r\left(M^{*}\right)=3$, so $M$ is spanned by $X$ and we have a contradiction.

First, we need to find an element $e$ of $M$ such that $M / e$ is near-regular.

Lemma 4.3.3. Let $M$ be a maximum-sized member of $\mathcal{N}$. Then there exists $e \in E(M)$ such that $M / e$ is near-regular.

Proof. Assume for a counterexample that no such $e$ exists. Then $M$ has a single-element extension $N$ by $f$, where $N$ is golden-mean and $N / f$ is nearregular. However, $M$ is maximum-sized, so $f$ must be parallel to an element $e$ of $M$. But then $M / e$ is near-regular, a contradiction.

Hence such an $e$ must exist. 
Now let $M$ be a maximum-sized counterexample to Theorem 4.1.2 of minimum rank. We will show that there are only two possible configurations for $\mathcal{L}(M, e)$. We start by restricting the lines through $e$ in $M$.

Corollary 4.3.4. Let $M$ be a counterexample to Theorem 4.1.2. Then $M$ has no conpunctual five-point lines.

Proof. If $M$ has no $B R$-minor, then we can apply Corollary 4.2.7 to acquire the desired result. It is now sufficient to show that $B R \notin \mathcal{N}$. To this end, we use the Sage code BR = matroids. named_matroids.BetsyRoss ( ) ; all([M.has_line_minor(5) for $M$ in [BR.contract(e) for e in

BR.groundset()]]), which outputs True, telling us that $B R / e$ contains a $U_{2,5}$-minor, and hence is not near-regular, for all $e \in E(B R)$. Since $B R$ is maximum-sized by Lemma 2.3.1 it follows that any golden-mean single-element extension of $B R$ is a parallel extension. Therefore this check suffices to show that $B R$ is not in $\mathcal{N}$.

Lemma 4.3.5. Let $M$ be a counterexample to Theorem 4.1 .2 of minimum rank, and assume that $M$ is maximum-sized. Let $e \in E(M)$ be such that $M / e$ is near-regular. If there exists an element $f$ in $\mathcal{L}(M, e)$ such that $\left|\mathrm{cl}_{M}(\{e, f\})\right| \geq 4$, then there can be no $U_{2,4}$ components in $\mathcal{L}(M, e)$.

Proof. Assume that there is a $U_{2,4}$ component $U$ of $\mathcal{L}(M, e)$. Lemma 4.1.32 states that $w_{u}=3$ for all $u \in U$. Let

$$
Z=\bigcup_{u \in U} \operatorname{cl}_{M}(\{e, u\}) \text { and } F=\operatorname{cl}_{M}(\{e, f\})
$$

As every element of $U \cup\{f\}$ is from $\mathcal{L}(M, e)$, it follows that $Z \cup F$ is a union of long lines from $M$. Note that $Z$ has rank three and consists of four copunctual three-point lines, and $F \notin \mathrm{cl}_{M}(Z)$.

Now, by Lemma 4.3.2, we can find a 3-connected minor $M^{\prime}$ of $M$, such that $M^{\prime}|(Z \cup F)=M|(Z \cup F)$ and $Z \cup F$ spans $M^{\prime}$. As $M^{\prime}$ is 3-connected, there must be an element $g \in E\left(M^{\prime}\right)$ such that $g \notin \mathrm{cl}_{M^{\prime}}(Z) \cup \mathrm{cl}_{M^{\prime}}(F)$.

Then $M^{\prime} / g$ contains a rank-three restriction containing four copunctual long lines, one of which is very long. Hence $M^{\prime} / g$ is not golden-mean by Lemma 2.3.1, a contradiction. 
Lemma 4.3.6. Let $M$ be a counterexample to Theorem 4.1 .2 of minimum rank, and assume that $M$ is maximum-sized. Let $e \in E(M)$ be such that $M / e$ is near-regular. If there exists an element $f$ in $\mathcal{L}(M, e)$ with $\left|\mathrm{cl}_{M}(\{e, f\})\right| \geq 4$, then there can be no $M\left(K_{4}\right)$ components in $\mathcal{L}(M, e)$.

Proof. Assume that there is a $M\left(K_{4}\right)$ component $K$ of $\mathcal{L}(M, e)$. Then Lemma 4.1.25 tells us that $w_{k}=3$ for all $k \in E(K)$. Let

$$
Z=\bigcup_{k \in K} \operatorname{cl}_{M}(\{e, k\}) \text { and } F=\operatorname{cl}_{M}(\{e, f\})
$$

As every element of $K \cup\{f\}$ is from $\mathcal{L}(M, e)$, it follows that $Z \cup F$ is a union of long lines from $M$. Note that $Z$ has rank four and consists of six copunctual three-point lines, and $F \notin \mathrm{cl}_{M}(Z)$.

Now, by Lemma 4.3.2, we can find a 3-connected minor $M^{\prime}$ of $M$, such that $M^{\prime}|(Z \cup F)=M|(Z \cup F)$ and $Z \cup F$ spans $M^{\prime}$. As $M^{\prime}$ is 3-connected, there must be an element $g \in E\left(M^{\prime}\right)$ such that $g \notin \mathrm{cl}_{M^{\prime}}(Z) \cup \mathrm{cl}_{M^{\prime}}(F)$.

Now $M^{\prime} / g / e$ contains a restriction $L$ spanned by $K$ such that $f \in$ $\mathrm{cl}_{M^{\prime} / g / e}(K)$. There are three possible locations for $f$ in $L$, relative to $K$ :

(i) Parallel to an element of $K$.

(ii) Placed such that $\operatorname{si}\left(M^{\prime} / g / e\right) \cong F_{7}^{=}$.

(iii) Placed such that $\operatorname{si}\left(M^{\prime} / g / e\right) \cong O_{7}$.

Hence we can find a four-element circuit $C$ of $\left(M^{\prime} / g\right) \mid L$, such that $f \in C$.

Let $I$ and $J$ be disjoint subsets of $E(M)$ such that $M \backslash I / J$ is equal to $\left(M^{\prime} / g / e\right) \mid C$. Let

$$
S=\bigcup_{c \in C} \mathrm{cl}_{M}(\{e, c\})
$$

Now $M \backslash(I-S) /(J-S)$ is a rank-four matroid containing four copunctual long lines. The rank of any three of these lines is four, but at least one of them contains four elements, contradicting Corollary 4.1.7.

We can now show that $\mathcal{L}(M, e)$ contains no $U_{2,4}$ or $M\left(K_{4}\right)$ components.

Corollary 4.3.7. Let $M$ be a counterexample to Theorem 4.1.2 of minimum rank, and assume that $M$ is maximum-sized. Let $e \in E(M)$ be such that $M / e$ is near-regular. Then there are no components in $\mathcal{L}(M, e)$ isomorphic to either $U_{2,4}$ or $M\left(K_{4}\right)$. 
Proof. If $\mathcal{L}(M, e)$ contains such a component, then by Lemmas 4.3.5 and 4.3.6, $w_{i}=3$ for all $i \in E(\mathcal{L}(M, e))$. Now it is easy to verify that the near-regular deficit of any component of $\mathcal{L}(M, e)$ is non-negative. This leads to a contradiction to (4.1.9).

We wish to restrict the values of $w_{i}$ in various cases.

Lemma 4.3.8. Let $M$ be a counterexample to Theorem 4.1 .2 of minimum rank, and assume that $M$ is maximum-sized. Let $e \in E(M)$ be such that $M / e$ is near-regular. Assume that $\mathcal{L}(M, e)$ has distinct components $N_{1}$ and $N_{2}$, both of which are isomorphic to $U_{2,3}$. Then there exists a four-element subset $X$ of $E\left(N_{1}\right) \cup E\left(N_{2}\right)$ with the property that $w_{x}=3$ for all $x \in X$.

Proof. Assume for a contradiction that the lemma is false. Then there exists $Y \subset E\left(N_{1}\right) \cup E\left(N_{2}\right)$ such that $|Y|=3$ and $w_{y}>3$ for all $y \in Y$. Let

$$
Z_{i}=\bigcup_{n \in E\left(N_{i}\right)} \mathrm{cl}_{M}(\{e, n\}) \text { for } i \in\{1,2\}
$$

As every element of $N_{1} \cup N_{2}$ is in $\mathcal{L}(M, e)$, it follows that $Z_{1} \cup Z_{2}$ is a union of long lines of $M$.

By Lemma 4.3.2, there is a 3-connected rank-five minor $M^{\prime}$ of $M$ such that $M\left|\left(Z_{1} \cup Z_{2}\right)=M^{\prime}\right|\left(Z_{1} \cup Z_{2}\right)$. Since $M^{\prime}$ is 3-connected, there is an element $g$ of $M^{\prime}$ that is in neither $\mathrm{cl}_{M^{\prime}}\left(Z_{1}\right)$ nor $\mathrm{cl}_{M^{\prime}}\left(Z_{2}\right)$.

Hence, in $M^{\prime} / g / e$, the lines $N_{1}$ and $N_{2}$ are coplanar. This means that we can find a four-element circuit $C$ of $M^{\prime} / g / e$ such that $C \subseteq E\left(N_{1}\right) \cup E\left(N_{2}\right)$, and $C \cap Y \neq \varnothing$. Let $I$ and $J$ be disjoint subsets of $E(M)$ such that $M \backslash I / J$ is $\left(M^{\prime} / g / e\right) \mid C$. Let

$$
S=\bigcup_{c \in C} \operatorname{cl}_{M}(\{e, c\})
$$

Now $M \backslash(I-S) /(J-S)$ is a rank-four matroid containing four copunctual long lines. The rank of any three of these lines is four, but at least one of them contains four elements, contradicting Corollary 4.1.7.

Lemma 4.3.9. Let $M$ be a counterexample to Theorem 4.1 .2 of minimum rank, and assume that $M$ is maximum-sized. Let $e \in E(M)$ be such that $M / e$ is near-regular. Then there is at most one component $N$ of $\mathcal{L}(M, e)$ such that $d_{n}(N)<0$. 
Proof. Assume that the lemma fails. By Corollaries $4.1 .24,4.1 .27,4.1 .31,4.1 .33$, and 4.1.35, the minimum near-regular deficit of any component of $\mathcal{L}(M, e)$ is -1 . It follows that there are components $N_{1}$ and $N_{2}$ of $\mathcal{L}(M, e)$ such that $d_{n}\left(N_{1}\right)=d_{n}\left(N_{2}\right)=-1$. By Corollaries 4.1.24, 4.1.27, 4.1.31, 4.1.33, and 4.1.35, $N_{1}$ and $N_{2}$ are isomorphic to either $U_{1,1}, U_{2,3}$, or $M\left(K_{2, b}^{+}\right)$for some $b \geq 2$.

Let $\{p, q\}=\{1,2\}$. Assume $N_{p}$ is isomorphic to $U_{2,3}$ and that $w_{i}<5$ for every element $i$ of $N_{p}$. By referring to Lemma 4.1.29, and the fact that $d_{n}\left(N_{p}\right)=-1$, we can assume that $w_{x}=w_{y}=4$ and $w_{z}=3$, where $\{x, y, z\}$ is the ground set of $N_{p}$.

Assume that there is an element $j$ in $N_{q}$ such that $w_{j}=5$. Let

$$
Z=\bigcup_{n \in N_{p}} \operatorname{cl}_{M}(\{e, n\}) \text { and } F=\operatorname{cl}_{M}(\{e, j\})
$$

As every element of $N_{p} \cup\{j\}$ is from $\mathcal{L}(M, e)$, it follows that $Z \cup F$ is a union of long lines from $M$. Note that $Z$ has rank three and consists of three copunctual long lines, two of which have length four, and $F \notin \mathrm{cl}_{M}(Z)$.

Now, by Lemma 4.3.2, we can find a 3-connected minor $M^{\prime}$ of $M$, such that $M^{\prime}|(Z \cup F)=M|(Z \cup F)$ and $Z \cup F$ spans $M^{\prime}$. As $M^{\prime}$ is 3-connected, there must be an element $g \in E\left(M^{\prime}\right)$ such that $g \notin \mathrm{cl}_{M^{\prime}}(Z) \cup \mathrm{cl}_{M^{\prime}}(F)$.

Then $M^{\prime} / g$ contains a rank-three restriction containing three copunctual long lines, one of length five, and one of length four. Hence $M^{\prime} / g$ is not goldenmean by Lemma 2.3.1, a contradiction, so no such $j$ can exist.

By considering Lemma 4.1.28 and the discussion prior to Corollary 4.1.34, we see that if $N_{q}$ is isomorphic to either $U_{1,1}$ or $M\left(K_{2, b}^{+}\right)$for some $b \geq 2$, then we have an element $j \in E\left(N_{q}\right)$ such that $w_{j}=5$, which we have just shown is impossible. Hence $N_{q} \cong U_{2,3}$, and Lemma 4.1.29 tells us that the three elements of $N_{q}$ have weights four, four, and three, respectively. This leads to a contradiction to Lemma 4.3.8.

Therefore neither $N_{p}$ nor $N_{q}$ is isomorphic to $U_{2,3}$, with all elements having weight strictly less than five. Now, as $d_{n}\left(N_{1}\right)=d_{n}\left(N_{2}\right)=-1$, we observe from Lemmas 4.1.28 and 4.1.29, and the discussion prior to Corollary 4.1.34, that there are points $a_{i} \in E\left(N_{i}\right)$ for $i \in\{1,2\}$ such that $w_{a_{i}}=5$. This contradicts Corollary 4.3.4, and we have completed the proof of the lemma. 
Corollary 4.3.10. Let $M$ be a counterexample to Theorem 4.1 .2 of minimum rank, and assume that $M$ is maximum-sized. Let $e \in E(M)$ be such that $M / e$ is near-regular. Then

$$
\sum_{N \lessdot \mathcal{L}(M, e)} d_{n}(N)=-1
$$

Proof. By (4.1.9), the sum is at most -1 . By Corollaries $4.1 .24,4.1 .27,4.1 .31,4.1 .33$, and 4.1.35, the minimum near-regular deficit of any component of $\mathcal{L}(M, e)$ is -1 . Now the result follows from Lemma 4.3.9.

Lemma 4.3.11. Let $M$ be a rank-r counterexample to Theorem 4.1.2 of minimum rank, and assume that $M$ is maximum-sized. Let $e \in E(M)$ be such that $M / e$ is near-regular. Then $\mathcal{L}(M, e)$ spans $M / e$, and $\operatorname{si}(M / e)$ is isomorphic to $T_{r-1}^{1}$.

Proof. First we prove that $\mathcal{L}(M, e)$ spans $M / e$, that is, $r^{\prime}=r(\mathcal{L}(M, e))=$ $r-1$. Note that

$$
r^{\prime} \leq r-1
$$

From Corollary 4.3.10 and the definition of Function 4.1.21 we can see that

$$
\begin{aligned}
-1 & =\sum_{N \lessdot \mathcal{L}(M, e)} d_{n}(N) \\
& =\sum_{N \lessdot \mathcal{L}(M, e)} 2 r(N)-\sum_{N \lessdot \mathcal{L}(M, e)} w(N) \\
& =2 r^{\prime}-\sum_{N \lessdot \mathcal{L}(M, e)} w(N) .
\end{aligned}
$$

When we combine (4.3.2) with (4.1.7) and (4.3.1), we deduce

$$
2 r-1 \leq \sum_{N \lessdot \mathcal{L}(M, e)} w(N)=2 r^{\prime}+1 \leq 2(r-1)+1,
$$

and thus equality holds throughout, and ergo $r^{\prime}=r-1$, as desired.

By considering (4.1.6), (4.1.8), and (4.3.2), along with the fact that $r^{\prime}=r-1$, we obtain

$$
2 r \leq \epsilon(M)-\epsilon(M / e)=\sum_{N \ll \mathcal{L}(M, e)} w(N)+1
$$




$$
=2 r^{\prime}+2=2(r-1)+2=2 r .
$$

As $M / e$ is near-regular, Theorem 1.1 .12 tells us that $\epsilon(M / e) \leq\left(\begin{array}{c}r+1 \\ 2\end{array}\right)-2$. Also, as $M$ is a counterexample to Theorem 4.1.2, $\epsilon(M) \geq\left(\begin{array}{c}r+3 \\ 2\end{array}\right)-5$. Now (4.3.3) tells us that we must have equality in both of these expressions, so $\operatorname{si}(M / e)$ is a maximum-sized near-regular matroid, and the result follows from Theorem 1.1.12.

We now prove some facts about restrictions of $T_{n}^{1}$. We first need to define some terminology related to $T_{n}^{k}$ for $k \in\{1,2\}$.

Definition 4.3.12. Let $k$ be 1 or 2 . The element of $T_{n}^{k}$ that is on $n-1$ lines of length $k+3$ is known as the acme of $T_{n}^{k}$. Each $(k+3)$-point line in $T_{n}^{k}$ is known as a leg. A contour of $T_{n}^{k}$ is a set of $n-1$ points (one from each leg), such that every pair of elements are on a line with a non-leg element.

Lemma 4.3.13. Assume $M$ is isomorphic to $T_{r}^{1}$, for some $r \geq 3$. Let $B$ be a restriction of $M$ that is isomorphic to $M\left(K_{2, b}^{+}\right)$where $b \geq 1$, and let $p$ be the head of $B$ (recall that if $b=1$, then $p$ is an arbitrary element of $B$ ). Let $x$ be an element of $M$ that is not in the span of $B$. Then there is a minor $M^{\prime}$ of $M$ such that

(i) $M^{\prime}|B=M| B$,

(ii) $B$ spans $M^{\prime}$,

(iii) $E\left(M^{\prime}\right)=E(B) \cup\{x\}$, and

(iv) $r_{M^{\prime}}(\{x, p\})=2$.

Proof. We prove this by induction on $r$. If $r=3$, then $b=1$. Up to symmetry there are three triangles of $M$ that could be $B$ :

1. a triangle that contains the acme of $M$,

2. a triangle that spans a leg of $M$ without containing the acme, and

3. the closure of a contour.

These are shown in Figure 4.5.

In all three cases, there are four or five points not in the span of $B$. By inspection, we see that by contracting points not in the span of $B$, any point not in the span of $B$ can be projected into at least two different locations in the span of $B$. This establishes the lemma when $r=3$. 


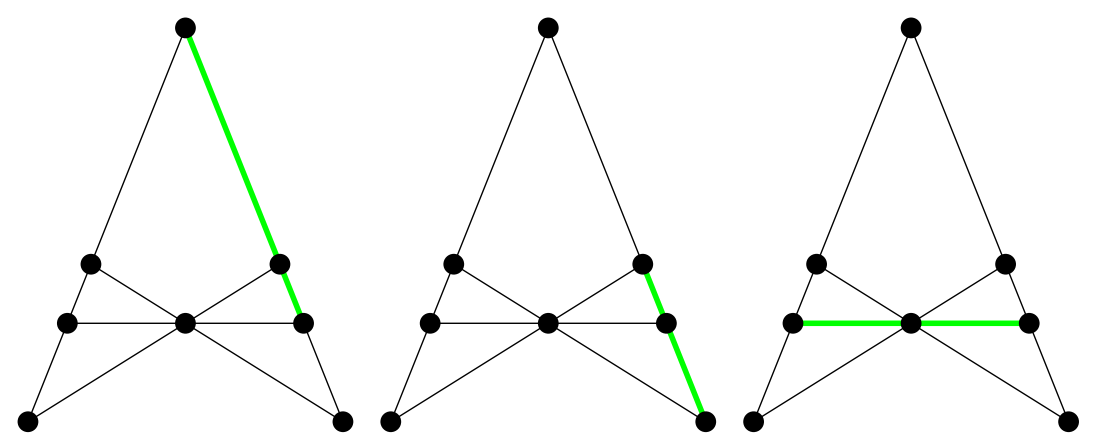

4.5.1: Case 1.

4.5.2: Case 2.

4.5.3: Case 3 .

Figure 4.5: Three triangles of $T_{3}^{1}$ for Lemma 4.3.13

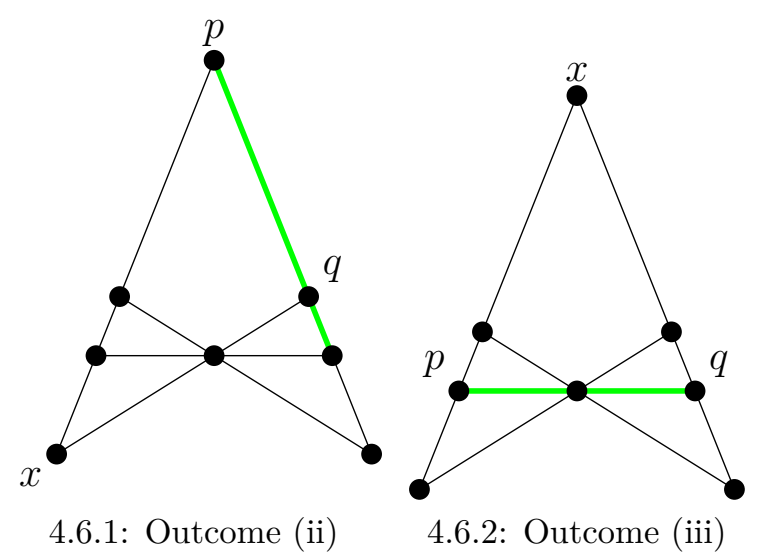

Figure 4.6: Outcomes (ii) and (iii) of Lemma 4.3.14

Now assume that $r>3$ and that the lemma holds for $r-1$. Since $B$ is not spanning in $M$, we can find a cocircuit $C^{*}$ such that $C^{*} \cap \mathrm{cl}_{M}(B)=$ $\varnothing$. In particular, we can assume that $x \in C^{*}$. Let $z$ be the acme of $M$, and let $P=\operatorname{cl}_{M}(\{p, x, z\})$. If $C^{*}$ is a subset of $P$, then $M$ contains a vertical 3-separation. However, this contradicts the fact that $T_{n}^{1}$ is vertically 4-connected [11, Lemma 5.1].

Therefore, we can let $y$ be an element of $C^{*} \backslash P$. Now si $(M / y) \cong T_{r-1}^{1}$, since $y \neq z$. Since $y$ is not an element of $\operatorname{cl}_{M}(B)$, it follows that $\operatorname{si}(M / y)|B=M| B$, so we can assume that $B \cup\{x\}$ is a subset of the ground set of $\operatorname{si}(M / y)$. If $x$ is in the closure of $B$ in $\operatorname{si}(M / y)$, then the restriction of $\operatorname{si}(M / y)$ to $B \cup\{x\}$ is the desired minor, as $x$ and $p$ are not parallel in si $(M / y)$. On the other hand, if $x$ is not in the closure of $B$, then we can apply the inductive hypothesis, and deduce that $M^{\prime}$ exists in any case. 
Lemma 4.3.14. Assume $M$ is isomorphic to $T_{r}^{1}$, for some $r \geq 3$. Let $B$ be a $U_{2,3}$-restriction of $M$ containing distinct elements $p$ and $q$. Let $x$ be an element of $E(M)$ that is not in the span of $B$. Then there is a minor $M^{\prime}$ of $M$ such that either

(i) $E\left(M^{\prime}\right)=B \cup\{x\}$, such that $B$ is a spanning circuit of $M^{\prime}$, and $x$ is parallel to neither $p$ or $q$, or

(ii) $M^{\prime}$ is isomorphic to $T_{3}^{1}$ such that $B$ is a circuit of $M^{\prime}$ with either $p$ or $q$ being the acme of $M^{\prime}$, and $x$ is the unique element of $M^{\prime}$ such that $\mathrm{cl}_{M^{\prime}}(\{x, p\})$ and $\mathrm{cl}_{M^{\prime}}(\{x, q\})$ are distinct long lines of $M^{\prime}$, or

(iii) $M^{\prime}$ is isomorphic to $T_{3}^{1}$ such that $B$ is the closure of a contour of $M^{\prime}$, and $x$ is the acme of $M^{\prime}$, while $p$ and $q$ are the points of $B$ that form a contour of $M^{\prime}$.

Up to symmetry, outcomes (ii) and (iii) are shown in Figure 4.6.

Proof. Let $M$ be a minimum-rank counterexample to the lemma, such that $r(M)=r$. If $r>3$, then there is a leg $\ell$ of $M$ that is not contained in $\operatorname{cl}_{M}(B \cup\{x\})$. Therefore there is a point $y$ of $\ell$ that is not in $\operatorname{cl}_{M}(B \cup\{x\})$ and that is not the acme of $M$. Hence $\operatorname{si}(M / y) \cong T_{r-1}^{1}$, and we can assume that $B \cup\{y\}$ is a subset of $E(\operatorname{si}(M / y))$. Therefore $\operatorname{si}(M / y)$ is a lower rank counterexample to the lemma, which is not possible. Ergo $r=3$.

Assume that $B$ is the closure of a contour of $M$, as shown in Figure 4.5.3. If $x$ is not the acme of $M$, then we let $y$ be an element of $E(M) \backslash B$ such that $y$ is not on a long line with $x$, and $y$ is not the acme of $M$. In this case, $B \cup\{x\}$ is a $U_{2,4}$-restriction in $M / y$, so outcome (i) holds. If $x$ is the acme and outcome (i) does not hold, then $\{p, q\}$ must be the intersection of $B$ and the legs of $M$, so outcome (iii) holds.

Now assume that $B$ spans a leg in $M$, but that $B$ does not contain the acme of $M$, as in Figure 4.5.2. Thus $x$ is not the acme of $M$. If $x$ is a non-leg element of $M$, then $x$ can be projected so that it becomes parallel with any of the three elements of $B$, and therefore outcome (i) occurs. If $x$ is a leg element of $M$, then it can be projected so that it becomes parallel with the acme, and hence outcome (i) holds again.

Finally, we assume that $B$ contains the acme, as shown in Figure 4.5.1. If $x$ is not a leg element of $M$, then we can easily verify that outcome (i) occurs. 
Therefore $x$ is a leg element of $M$, and the two points that are in $\operatorname{cl}_{M}(B)$ and also on long lines with $x$ must be $p$ and $q$, and ergo outcome (ii) occurs.

We are now able to show that there are exactly two configurations of $\mathcal{L}(M, e)$.

Lemma 4.3.15. Let $M$ be a rank-r counterexample to Theorem 4.1.2 of minimum rank, and assume that $M$ is maximum-sized. Let $e \in E(M)$ be such that $M /$ e is near-regular. Then $\mathcal{L}(M, e)$ is either

(i) a collection of coloops, or

(ii) isomorphic to $M\left(K_{2, r-2}^{+}\right)$.

Proof. By Lemma 4.3.9 and Corollary 4.3.10, there is a single component of $\mathcal{L}(M, e)$ with near-regular deficit -1 , and all other components must have a near-regular deficit of zero. Let $N_{1}, \ldots, N_{t}$ be the components of $\mathcal{L}(M, e)$ where $d_{n}\left(N_{1}\right)=-1$. From Corollaries 4.1.24, 4.1.27, 4.1.31, 4.1.33, 4.1.35, and 4.3.7, we see that each $N_{i}$ is isomorphic to $U_{1,1}, U_{2,3}$, or $M\left(K_{2, b}^{+}\right)$where $b \geq 2$.

Assume $N_{1}$ is isomorphic to $U_{2,3}$ and $w_{n}<5$ for all $n \in E\left(N_{1}\right)$. Therefore, by Lemma 4.1 .29 , we can assume that $w_{x}=w_{y}=4$ and $w_{z}=3$, where $\{x, y, z\}$ is the ground set of $N_{1}$. Since $r>3$, the rank of $\mathcal{L}(M, e)$ is at least three by Lemma 4.3.11, so $t>1$. Since $d_{n}\left(N_{2}\right)=0$, it follows from Lemmas 4.1.28 and 4.1.29, and the discussion prior to Corollary 4.1.34, that there is no element in $N_{2}$ with weight five, but there must be an element $a_{2}$ with weight four. We can assume that $a_{2}, x, y$, and $z$ are elements of $E(\operatorname{si}(M / e))$, and we apply Lemma 4.3 .14 to this matroid. It follows that $M$ has a minor $N$ such that either $N$ is isomorphic to $U_{2,3}$ and contains $x, y$, and $a_{2}$, or $N$ is isomorphic to $T_{3}^{1}$, where $x, y, z$, and $a_{2}$ are as in one of the configurations detailed by Lemma 4.3.14 and shown in Figure 4.6.

Let $I$ and $J$ be disjoint subsets of $M$ such that $M \backslash I / J$ is $N$. Let

$$
Z=\bigcup_{n \in E(N)} \operatorname{cl}_{M}(\{e, n\})
$$

and consider $M \backslash(I-Z) /(J-Z)$. If $N$ is isomorphic to $U_{2,3}$, then $M \backslash(I-$ $Z) /(J-Z)$ is a rank-three matroid containing three copunctual very long lines, and hence is not golden-mean by Lemma 2.3.1. In the other two cases, $M \backslash(I-Z) /(J-Z)$ must be discovered by a computer search, using the Sage 
code from Appendix 4.A.3. However, this computer search reports that no such golden-mean matroids exist.

Now we see that $N_{1}$ is isomorphic to either $U_{1,1}$ or $M\left(K_{2, b}^{+}\right)$where $b \geq 1$. In the latter case if $b \geq 2$, then, by Lemma 4.1 .28 , the head of $M\left(K_{2, b}^{+}\right)$is weighted five and all other elements of $M\left(K_{2, b}^{+}\right)$are weighted three. If $b=1$, then, by the previous paragraphs and Lemma 4.1.29, exactly one element, known as the head of $M\left(K_{2,1}^{+}\right)$, is weighted five, and the other two elements of $M\left(K_{2,1}^{+}\right)$are weighted three.

If the statement of the lemma is false, then one of the following situations occurs:

(i) $N_{1}$ is isomorphic to $U_{1,1}$, and, without loss of generality, $N_{2}$ is isomorphic to $M\left(K_{2, b}^{+}\right)$for some $b \geq 1$.

(ii) $N_{1}$ is isomorphic to $M\left(K_{2, b}^{+}\right)$for some $b \geq 1$, and, without loss of generality, $N_{2}$ is isomorphic to $M\left(K_{2, d}^{+}\right)$for some $d \geq 1$.

(iii) $N_{1}$ is isomorphic to $M\left(K_{2, b}^{+}\right)$for some $b \geq 1$, and, without loss of generality, $N_{2}$ is isomorphic to $U_{1,1}$.

We go through each case in turn and show that they are contradictory.

Sublemma 4.3.15.1. Case (i) is contradictory.

Subproof. Let $X$ be a subset of $E(M / e)$ such that $M / e \backslash X \cong \operatorname{si}(M / e)$, and let $M_{e}=M / e \backslash X$. By Lemma 4.3.11, we know that $M_{e} \cong T_{r-1}^{1}$. By the definition of case (i), we know that $M_{e}$ has a $M\left(K_{2, b}^{+}\right)$restriction $B$ for some $b \geq 1$. Furthermore, $w(B)=0$, and so Lemmas 4.1.28 and 4.1.29 tell us that the weight of every non-head element in $B$ is three, while the head, $p$, of $B$ is weighted four.

Now, there must be an element $x$ of $M_{e}$ such that $\left|\mathrm{cl}_{M}(\{e, x\})\right|=5$. Hence we can apply Lemma 4.3 .13 to find a minor $M^{\prime}$ of $M_{e}$ such that $B$ spans $M^{\prime}$ and $E\left(M^{\prime}\right)=E(B) \cup\{x\}$, where $x$ is not in parallel with $p$. We can now find a rank-two minor $M^{\prime \prime}$ of $M^{\prime}$ with at least three elements such that $r_{M^{\prime \prime}}(\{x, p\})=2$.

Consider the matroid $M_{Z}$ spanned by $Z$, where

$$
Z=\bigcup_{z \in E\left(M^{\prime \prime}\right)} \operatorname{cl}_{M}(\{e, z\})
$$


Now, $M_{Z}$ is a rank-three minor of $M$ containing at least three long lines containing $e$, one of length five, and one of length four. This leads to a contradiction to Lemma 2.3.1, and so case (i) is contradictory, as required.

Sublemma 4.3.15.2. Case (ii) is contradictory.

Subproof. By previous work, we know that $p$, the head of $N_{1}$, is weighted five. Consider the restriction of $\mathcal{L}(M, e)$ where $N_{1}$ is restricted to $p$, and apply Sublemma 4.3.15.1. Hence case (ii) is contradictory.

Sublemma 4.3.15.3. Case (iii) is contradictory.

Subproof. This is very similar to the proof of Sublemma 4.3.15.1.

As all three cases are contradictory, the lemma must be true.

From Lemma 4.3.15, we can deduce the values of $\left|\mathrm{cl}_{M}(\{e, x\})\right|$ for all $x \in$ $E(\mathcal{L}(M, e))$. The next two corollaries make this explicit.

Corollary 4.3.16. Let $M$ be a rank-r counterexample to Theorem 4.1 .2 of minimum rank, and assume that $M$ is maximum-sized. Let $e \in E(M)$ be such that $M / e$ is near-regular. If $\mathcal{L}(M, e)$ is a collection of coloops, then there is one coloop $y$ such that $\left|\mathrm{cl}_{M}(\{e, y\})\right|=5$, and $\left|\mathrm{cl}_{M}(\{e, x\})\right|=4$ for all $x \in E(\mathcal{L}(M, e)) \backslash\{y\}$.

Corollary 4.3.17. Let $M$ be a rank-r counterexample to Theorem 4.1 .2 of minimum rank, and assume that $M$ is maximum-sized. Let $e \in E(M)$ be such that $M /$ e is near-regular. If $\mathcal{L}(M, e)$ is isomorphic to $M\left(K_{2, b}^{+}\right)$, then $\left|\mathrm{cl}_{M}(\{e, p\})\right|=5$ where $p$ is the head of $M\left(K_{2, b}^{+}\right)$, and $\left|\mathrm{cl}_{M}(\{e, x\})\right|=3$ for all $x \in E(\mathcal{L}(M, e)) \backslash\{p\}$.

Lemma 4.3.11 tells us that $\operatorname{si}(M / e) \cong T_{r-1}^{1}$, and Lemma 4.3.15 states that there are two possible solutions for $\mathcal{L}(M, e)$. Therefore we split the remainder of the proof of Theorem 4.1.2 into two natural cases. 


\subsubsection{All coloops}

The first case to consider is when $\mathcal{L}(M, e)$ is a collection of coloops. In this case, Corollary 4.3.16 tells us exactly what the long lines containing $e$ are.

Lemma 4.3.18. Let $M$ be a counterexample to Theorem 4.1.2 of minimum rank, and assume that $M$ is maximum-sized. Let $e \in E(M)$ be such that $M / e$ is near-regular. If $\mathcal{L}(M, e)$ is a collection of coloops, then $\mathcal{L}(M, e)$ is a basis of $M / e$.

Proof. $\mathcal{L}(M, e)$ and $M / e$ have the same rank. The result follows as $\mathcal{L}(M, e)$ is a collection of coloops.

We now show that $\mathcal{L}(M, e)$ must be a specific basis.

Lemma 4.3.19. Let $M$ be a counterexample to Theorem 4.1.2 of minimum rank, and assume that $M$ is maximum-sized. Let $e \in E(M)$ be such that $M / e$ is near-regular. If $\mathcal{L}(M, e)$ is a collection of coloops, then the basis of $\operatorname{si}(M / e)$ corresponding to $\mathcal{L}(M, e)$ can have no fundamental circuit of size greater than three.

Proof. Let $B$ be this basis of $\operatorname{si}(M / e)$, and let $x$ be an element of $\operatorname{si}(M / e)$ that is not in $B$. If $C \subseteq B \cup\{x\}$ is a fundamental circuit such that $|C| \geq 4$, then when we contract $x$ and all but three points in $C$ from $\operatorname{si}(M / e) / x$, we get a three-point line, such that each point was from $B$. This leads to a rank-three restriction of $M$, with three copunctual four-point lines, a contradiction to Lemma 2.3.1.

Lemma 4.3.20. The only basis of $T_{n}^{1}$ with all fundamental circuits having size three is the basis consisting of the acme of $T_{n}^{1}$ and a contour.

Proof. We prove this by induction.

The lemma is true by inspection when $n=3$, so now assume $n>3$. Let $B$ be a basis of $T_{n}^{1}$. Assume that $B$ contains no leg element, apart from possibly the acme. If $e$ is an arbitrary leg element, then there is a line spanned by two elements of $B$ that contains $e$. This line cannot contain the acme, so it consists of $e$, a non-leg element, and another leg element. Now we have a contradiction to the assumption that $B$ contains no leg elements. 
Hence we may assume that $e$ in $B$ is a leg element. In $\operatorname{si}(M / e)$, the fundamental circuits of $B \backslash\{e\}$ have size at most three, and $\operatorname{si}(M / e)$ is isomorphic to $T_{n-1}^{1}$, so by induction, $B \backslash\{e\}$ consists of the acme, and a contour. If we pick $f$ in this contour, then $B \backslash\{f\}$ consists of the acme and a contour, and it follows without difficulty that $B$ consists of the acme and a contour of $T_{n}^{1}$. $\square$

Corollary 4.3.21. Let $M$ be counterexample to Theorem 4.1.2 of minimum rank, and assume that $M$ is maximum-sized. Let $e \in E(M)$ be such that $M / e$ is near-regular. If $\mathcal{L}(M, e)$ is a collection of coloops, then $\mathcal{L}(M, e)$ is the acme of $\operatorname{si}(M / e)$ and a contour.

Proof. This is an amalgamation of Lemmas 4.3.18, 4.3.19, and 4.3.20.

Corollary 4.3.16 states that there is one distinguished coloop in $\mathcal{L}(M, e)$, namely the coloop $x$ such that $\left|\operatorname{cl}_{M}(\{e, x\})\right|=5$. We now show that $x$ must correspond to the acme of $\operatorname{si}(M / e)$.

Lemma 4.3.22. Let $M$ be a rank-r counterexample to Theorem 4.1 .2 of minimum rank, and assume that $M$ is maximum-sized. Let $e \in E(M)$ be such that $M /$ e is near-regular. If $\mathcal{L}(M, e)$ is a collection of coloops, then the coloop $x \in \mathcal{L}(M, e)$ such that $\left|\mathrm{cl}_{M}(\{e, x\})\right|=5$ corresponds to the acme of $\operatorname{si}(M / e)$.

Proof. Lemma 4.3.11 tells us that $\operatorname{si}(M / e) \cong T_{r-1}^{1}$, so the acme of $\operatorname{si}(M / e)$ exists. Furthermore, Corollary 4.3.21 tells us that $\mathcal{L}(M, e)$ is an acme and a contour of $\operatorname{si}(M / e)$.

Let $\ell_{i}$ be $\mathrm{cl}_{M}(\{e, i\})$ for $i \in E(\mathcal{L}(M, e))$, and let $a$ be the acme of $\operatorname{si}(M / e)$.

Assume that $x \neq a$. Then $\operatorname{cl}_{M}(\{e, a, x\})$ is a rank-three flat $F_{a x}$ of $M$ with ten elements, containing a four-point line $\left(\ell_{a}\right)$ and a five-point line $\left(\ell_{x}\right)$ that meet at $e$. Lemma 2.3.1 implies that $F_{a x} \cong G_{3}$.

Let $N$ be a simple minor of $M$ containing $F_{a x}$ such that $N\left|F_{a x}=M\right| F_{a x}$ and $\left|\mathrm{cl}_{N}\left(\ell_{a}\right)\right|=5$. This means that $\mathrm{cl}_{N}(\{e, a, x\})$ is an eleven-point rank-three flat with a five-point line restriction. This is a contradiction to Lemma 2.3.1, so such an $N$ cannot exist.

Let $y$ be an element of $E(\mathcal{L}(M, e)) \backslash\{a, x\}$. Then $\operatorname{cl}_{M}(\{e, a, y\})$ is a ninepoint rank-three flat $F_{a y}$ of $M$ containing $\ell_{a}$. By analysis of the matroids listed in Lemma 2.3.1, we see that, in this case, we can contract an element 
from $F_{a y} \backslash \ell_{a}$ and project a fifth point onto $\ell_{a}$. Since $\left(F_{a y} \backslash \ell_{a}\right) \cap F_{a x}=\varnothing$, we have produced a minor $N$ of the type described in the previous paragraph, a contradiction.

Hence $x=a$, as desired.

We now have enough information to prove that Conjecture 1.2.3 is true in this case.

Lemma 4.3.23. Let $M$ be a rank-r counterexample to Theorem 4.1 .2 of minimum rank, and assume that $M$ is maximum-sized. Let $e \in E(M)$ be such that $M /$ e is near-regular. Then $\mathcal{L}(M, e)$ cannot be a collection of coloops.

Proof. We show that if $\mathcal{L}(M, e)$ is a collection of coloops, then $M \cong G_{r}$.

We are going to construct a $G F(4)$-representation for $M$. We know that $e$ is on $r-1$ long lines, one of length five, and $r-2$ of length four. Let $\ell_{1}, \ldots, \ell_{r-1}$ be these long lines, where $\ell_{1}$ has length five.

From Lemma 4.3.11, we know that $\operatorname{si}(M / e) \cong T_{r-1}^{1}$. Let $f_{i}$ be the element of $\mathcal{L}(M, e)$ that is also in $\ell_{i}$, for $i \in\{1, \ldots, r-1\}$.

As we have uniqueness of representation over $G F(4)$ by Lemma 2.1.12, we can assume that $M$ is represented by a matrix of the form

$$
\left[\begin{array}{l|l|l}
I_{r} & A & * \cdots * \\
\cline { 2 - 2 } & T_{r-1}
\end{array}\right]
$$

where the first $r$ columns are labelled by $\left(e, f_{1}, \ldots, f_{r-1}\right)$, and $T_{r-1}$ is the matrix

$$
\left[\begin{array}{c|c|c}
1 \cdots 1 & \alpha \cdots \alpha & 0 \cdots 0 \\
\hline I_{r-2} & I_{r-2} & D_{r-2}
\end{array}\right]
$$

The labelling is correct as Corollary 4.3.21 and Lemma 4.3.22 tell us exactly where $\mathcal{L}(M, e)$ is located in $\operatorname{si}(M / e)$.

Since $f_{1}$ is on a five-point line with $e$, and $e$ is on a four-point line with all other $f_{i}$ s, we get that $M$ must be represented by a matrix shown in Figure 4.7.1, where each $\sharp$ is an element of $G F(4)$ and each $*$ is a non-zero element of $G F(4)$. 

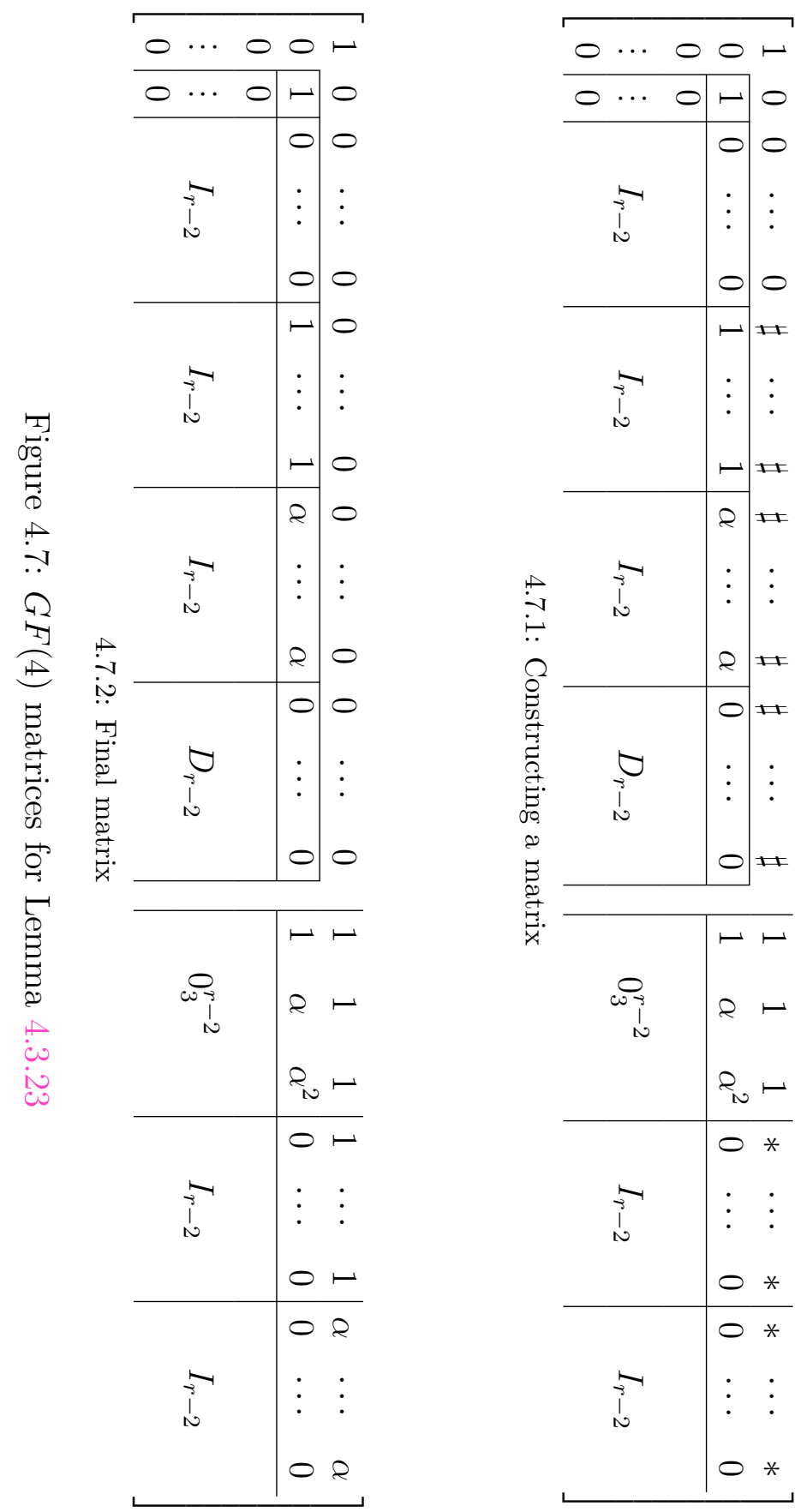
Now consider the matroid $\operatorname{si}\left(M / f_{r-1}\right)$. Upon considering the matrix of $M$, we see that $\epsilon(M)-\epsilon\left(M / f_{r-1}\right) \leq r+2$. However, we know that $\epsilon(M)-$ $\epsilon\left(M / f_{r-1}\right) \geq r+2$, and hence $\epsilon(M)-\epsilon\left(M / f_{r-1}\right)=r+2$, so si $\left(M / f_{r-1}\right) \in \mathscr{G}_{r-1}$.

When we look at the matrix for $\operatorname{si}\left(M / f_{r-1}\right)$, we see a solitary five-point line and at least one four-point line. From the descriptions of the members of $\mathscr{G}_{r-1}$ in Section 2.2.1, we note that $G_{r-1}$ is the only member of $\mathscr{G}_{r-1}$ having the required lines. Hence $\operatorname{si}\left(M / f_{r-1}\right) \cong G_{r-1}$, and we are able to see that $\sharp=0$. Also, we see that all except the last $*$ in each block are also fixed. To discover the last two values of $*$, we repeat this argument with $f_{r-2}$.

Hence $M$ must be represented by the matrix in Figure 4.7.2, which is identical to the $G F(4)$ matrix for $G_{r}$ given in Figure 2.5.2. Hence $M \cong G_{r}$.

Hence $\mathcal{L}(M, e)$ cannot be a collection of coloops.

\subsection{2 $M\left(K_{2, r-2}^{+}\right)$}

As $\mathcal{L}(M, e)$ cannot be a collection of coloops by Lemma 4.3.23, we can modify Lemma 4.3.15 to get that $\mathcal{L}(M, e) \cong M\left(K_{2, r-2}^{+}\right)$.

Corollary 4.3.24. Let $M$ be a rank-r counterexample to Theorem 4.1 .2 of minimum rank, and assume that $M$ is maximum-sized. Let $e \in E(M)$ be such that $M /$ e is near-regular. Then $\mathcal{L}(M, e)$ is isomorphic to $M\left(K_{2, r-2}^{+}\right)$.

Recall from Corollary 4.3.17 that the head of $\mathcal{L}(M, e)$ corresponds to a fivepoint line of $M$, and every other point in $\mathcal{L}(M, e)$ corresponds to a three-point line of $M$.

We wish to know how $\mathcal{L}(M, e)$ is embedded inside of $\operatorname{si}(M / e)$.

Lemma 4.3.25. Let $M$ be a counterexample to Theorem 4.1 .2 of minimum rank, and assume that $M$ is maximum-sized. Let $e \in E(M)$ be such that $M / e$ is near-regular. Then the head of $\mathcal{L}(M, e)$ must be identified with a leg element of $\operatorname{si}(M / e)$.

Proof. Let $p$ be the head of $\mathcal{L}(M, e)$. For a contradiction, assume that $p$ is a non-leg element of $\operatorname{si}(M / e)$. Let $I$ and $J$ be disjoint subsets of $E(M / e)$ such that $M \backslash I / J=N$, where $N$ is a $T_{3}^{1}$ restriction of $\operatorname{si}(M / e)$ spanned by a $M\left(K_{2,2}^{+}\right)$restriction $K$ of $\mathcal{L}(M, e)$. Note that $p \in E(N)$. 
Such an $N$ exists as $\mathcal{L}(M, e)$ has $r-2$ limbs, and a non-leg element of $T_{k}^{1}$ is on exactly $r-2$ long lines, hence the $T_{3}^{1}$-restriction of $\operatorname{si}(M / e)$ that contains $p$ must have two limbs of $\mathcal{L}(M, e)$ contained within it.

As $p$ is a non-leg element of $N$, up to symmetry, there is only one place that $K$ could go in $N$, namely consisting of two contours of $N$ and $p$. Let $f$ be a non-acme element of $E(N) \backslash E(K)$. Let

$$
Z=\bigcup_{x \in E(\mathcal{L}(M, e))} \mathrm{cl}_{M}(\{e, x\})
$$

Now consider the matroid $X=\operatorname{si}(M \backslash(I-Z) /(J-Z) / f)$. In $X$, which has rank three, there are eleven points, five of which lie on a line. This is a contradiction to Lemma 2.3.1.

Hence the head of $\mathcal{L}(M, e)$ must be identified with a leg element of $\operatorname{si}(M / e)$.

Lemma 4.3.26. Let $M$ be a rank-r counterexample to Theorem 4.1 .2 of minimum rank, and assume that $M$ is maximum-sized. Let $e \in E(M)$ be such that $M / e$ is near-regular. Then the head of $\mathcal{L}(M, e)$ must be identified with the acme of $\operatorname{si}(M / e)$.

Proof. Let $p$ be the head of $\mathcal{L}(M, e)$. From Lemma 4.3.25, we know that $p$ must be identified with a leg element of $\operatorname{si}(M / e)$. Assume for a contradiction that $p$ is not the acme of $\operatorname{si}(M / e)$.

As in the proof of Lemma 4.3.25, let $N$ be a rank-three flat of $\operatorname{si}(M / e)$ spanned by a $M\left(K_{2,2}^{+}\right)$restriction $K$ of $\mathcal{L}(M, e)$. Note that $p \in E(N)$, and that $N \cong T_{3}^{1}$.

Up to symmetry, there are two possible locations for $K$ inside of $N$. One limb of $K$ must be the closure of a contour containing $p$, and the other limb of $K$ must be a subset $S$ of the leg of $N$ containing $p$.

If $S$ contains the acme of $N$, then let $f$ be the element of $N$ that is

(i) not in a contour with any element of $K$, and

(ii) not on the same leg of $N$ as $p$.

If $S$ does not contain the acme of $N$, then let $f$ be an element of $N \backslash K$ that is not the acme of $N$.

In both cases, when we consider $\operatorname{si}(M / f)$, we can see an eleven-element rank- 
three minor of $M$ containing a five-point line, which is a contradiction to Lemma 2.3.1.

As all other possibilities have been rejected, the head of $\mathcal{L}(M, e)$ must be identified with the acme of $\operatorname{si}(M / e)$.

Lemma 4.3.27. Let $M$ be a rank-r counterexample to Theorem 4.1 .2 of minimum rank, and assume that $M$ is maximum-sized. Let $e \in E(M)$ be such that $M / e$ is near-regular. Then the non-head elements of $\mathcal{L}(M, e)$ must be identified with two contours of $\operatorname{si}(M / e)$.

Proof. Assume for a contradiction that this lemma is false. Then there exists a rank-three restriction $N$ of $\operatorname{si}(M / e)$ that is isomorphic to $T_{3}^{1}$ and has $\mathcal{L}(M, e)$ embedded in such a way that $K=\mathcal{L}(M, e) \mid N$ does not contain two contours of $N$.

There is one non-leg element $f$ of $N$. When we consider $\operatorname{si}(M / f)$, we can see an eleven-element rank-three minor of $M$ containing a five-point line, which is a contradiction to Lemma 2.3.1.

Hence the non-head elements of $\mathcal{L}(M, e)$ must be identified with two contours in $\operatorname{si}(M / e)$.

Recall the definition of $T_{r}^{1}$ from Page 60.

Note that in $T_{r}^{1}$ there are three contours: one belongs to the distinguished basis, and is known as the basis contour, and the other two (headed by 1 and $\alpha$ in the matrix of $T_{r}^{1}$ ) are known as non-basis contours.

The next two lemmas, whose proofs are similar to the proof of Lemma 4.3.23, show that Theorem 4.1.2 is true.

Lemma 4.3.28. Let $M$ be a rank-r counterexample to Theorem 4.1 .2 of minimum rank, and assume that $M$ is maximum-sized. Let $e \in E(M)$ be such that $M / e$ is near-regular. Then $\mathcal{L}(M, e)$ cannot contain the basis contour of $\operatorname{si}(M / e)$.

Proof. We show that if $\mathcal{L}(M, e)$ does contain the basis contour of $\operatorname{si}(M / e)$, then $M \cong H P_{r}$. So assume for a contradiction that $\mathcal{L}(M, e)$ contains the basis contour of $\operatorname{si}(M / e)$. 
We are going to construct a $G F(4)$-representation of $M$. We know that $e$ is on $2 r-3$ long lines, one of length five and $2(r-2)$ of length three. Let $\ell_{1}, \ldots, \ell_{2 r-3}$ be these long lines, where $\ell_{1}$ has length five, and $\ell_{2}, \ldots, \ell_{r-1}$ correspond to the basis contour of $\operatorname{si}(M / e)$.

From Lemma 4.3.11, we know that $\operatorname{si}(M / e) \cong T_{r-1}^{1}$. Let $f_{i}$ be the element of $\mathcal{L}(M, e)$ that is also in $\ell_{i}$, for $i \in\{1, \ldots, 2 r-3\}$.

As we have uniqueness of representation over $G F(4)$ by Lemma 2.1.12, we can assume that $M$ is represented by a matrix of the form

$$
\left[\begin{array}{c|c|c}
I_{r} & A & * \cdots * \\
\cline { 1 - 2 } & T_{r-1}
\end{array}\right]
$$

where the first $r$ columns are labelled by $\left(e, f_{1}, \ldots, f_{r-1}\right)$, and $T_{r-1}$ is the matrix

$$
\left[\begin{array}{c|c|cc}
1 \cdots 1 & \alpha \cdots \alpha & 0 \cdots 0 \\
\hline I_{r-2} & I_{r-2} & D_{r-2}
\end{array}\right] .
$$

The labelling is correct as we are assuming that $\mathcal{L}(M, e)$ contains the acme and the basis contour of $\operatorname{si}(M / e)$.

Since $f_{1}$ is on a five-point line with $e$, and $e$ is on a three-point line with all other $f_{i}$ s, we get that $M$ must be represented by a matrix shown in Figure 4.8.1, where each $\sharp$ is an element of $G F(4)$ and each $*$ is a non-zero element of $G F(4)$.

Now consider the matroid $\operatorname{si}\left(M / f_{r-1}\right)$. Upon considering the matrix of $M$, we see that $\epsilon(M)-\epsilon\left(M / f_{r-1}\right) \leq r+2$. However, we know that $\epsilon(M)-$ $\epsilon\left(M / f_{r-1}\right) \geq r+2$, and hence $\epsilon(M)-\epsilon\left(M / f_{r-1}\right)=r+2$, so si $\left(M / f_{r-1}\right) \in \mathscr{G}_{r-1}$.

When we look at the matrix for $\operatorname{si}\left(M / f_{r-1}\right)$, we see a solitary five-point line and no four-point lines. Hence $\operatorname{si}\left(M / f_{r-1}\right) \cong H P_{r-1}$, and we are able to determine the values for $\sharp$. Also, we see that all except the last $*$ in each block are also fixed. To discover the last two values of $*$, we repeat this argument with $f_{r-2}$.

Hence $M$ must be represented by the matrix in Figure 4.8.2, which is identical to the $G F(4)$ matrix for $H P_{r}$ given in Figure 2.5.3. Hence $M \cong H P_{r}$. 

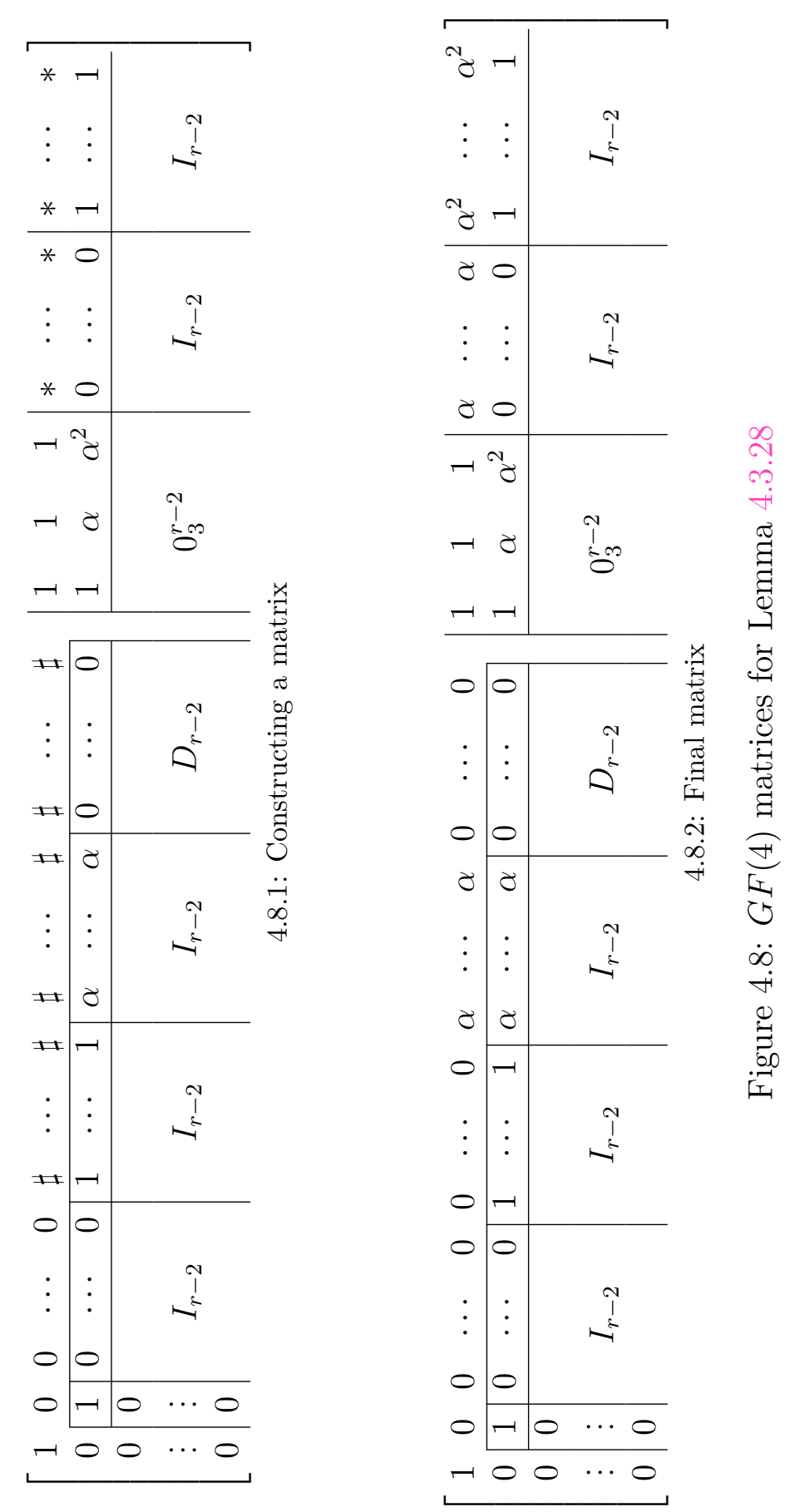
Therefore $\mathcal{L}(M, e)$ cannot contain the basis contour of $\operatorname{si}(M / e)$.

We now know exactly how $\mathcal{L}(M, e)$ is embedded in $\operatorname{si}(M / e)$.

Corollary 4.3.29. Let $M$ be a rank-r counterexample to Theorem 4.1 .2 of minimum rank, and assume that $M$ is maximum-sized. Let $e \in E(M)$ be such that $M /$ e is near-regular. Then $\mathcal{L}(M, e)$ is isomorphic to $M\left(K_{2, r-2}^{+}\right)$, with the head of $\mathcal{L}(M, e)$ identified with the acme of $\operatorname{si}(M / e)$, and the limbs of $\mathcal{L}(M, e)$ correspond to the two non-basis contours of $\operatorname{si}(M / e)$.

We can now prove Theorem 4.1.2. Let $M$ be a rank- $r$ counterexample to Theorem 4.1.2 of minimum rank. We can assume that $M$ is maximum-sized. By Lemma 4.3.3, we can find $e \in E(M)$ such that $M / e$ is near-regular.

We are going to construct a $G F(4)$ representation for $M$. By Corollary 4.3.17, we know there are exactly $2 r-3$ long lines passing through $e$, one of length five, and $2(r-2)$ of length three. Lemma 4.3 .11 tells us that $\operatorname{si}(M / e) \cong T_{r-1}^{1}$, and Corollary 4.3.29 tells us how $\mathcal{L}(M, e)$ is embedded in $\operatorname{si}(M / e)$.

Let $\ell_{1}, \ldots, \ell_{2 r-3}$ be the long lines of $M$ that contain $e$, where $\ell_{1}$ is the line of length five. Let $f_{i}$ be the element of $\mathcal{L}(M, e)$ that is also in $\ell_{i}$, for $i \in$ $\{1, \ldots, 2 r-3\}$.

As we have uniqueness of representation over $G F(4)$ by Lemma 2.1.12, we can assume that $M$ is represented by a matrix of the form

$$
\left[\begin{array}{c|c|c}
I_{r} & A & * \cdots * \\
\cline { 2 - 2 } & T_{r-1}
\end{array}\right]
$$

where the first $r$ columns are labelled by $\left(e, f_{1}, g_{1}, \ldots, g_{r-2}\right)$, and $T_{r-1}$ is the matrix

$$
\left[\begin{array}{c|c|cc}
1 \cdots 1 & \alpha \cdots \alpha & 0 \cdots 0 \\
\hline I_{r-2} & I_{r-2} & D_{r-2}
\end{array}\right] .
$$

with the first $2(r-2)$ columns labelled by $f_{2}, \ldots, f_{2 r-3}$.

The labelling is correct as we know that $\mathcal{L}(M, e)$ consists of the acme and the two non-basis contours of $\operatorname{si}(M / e)$. 

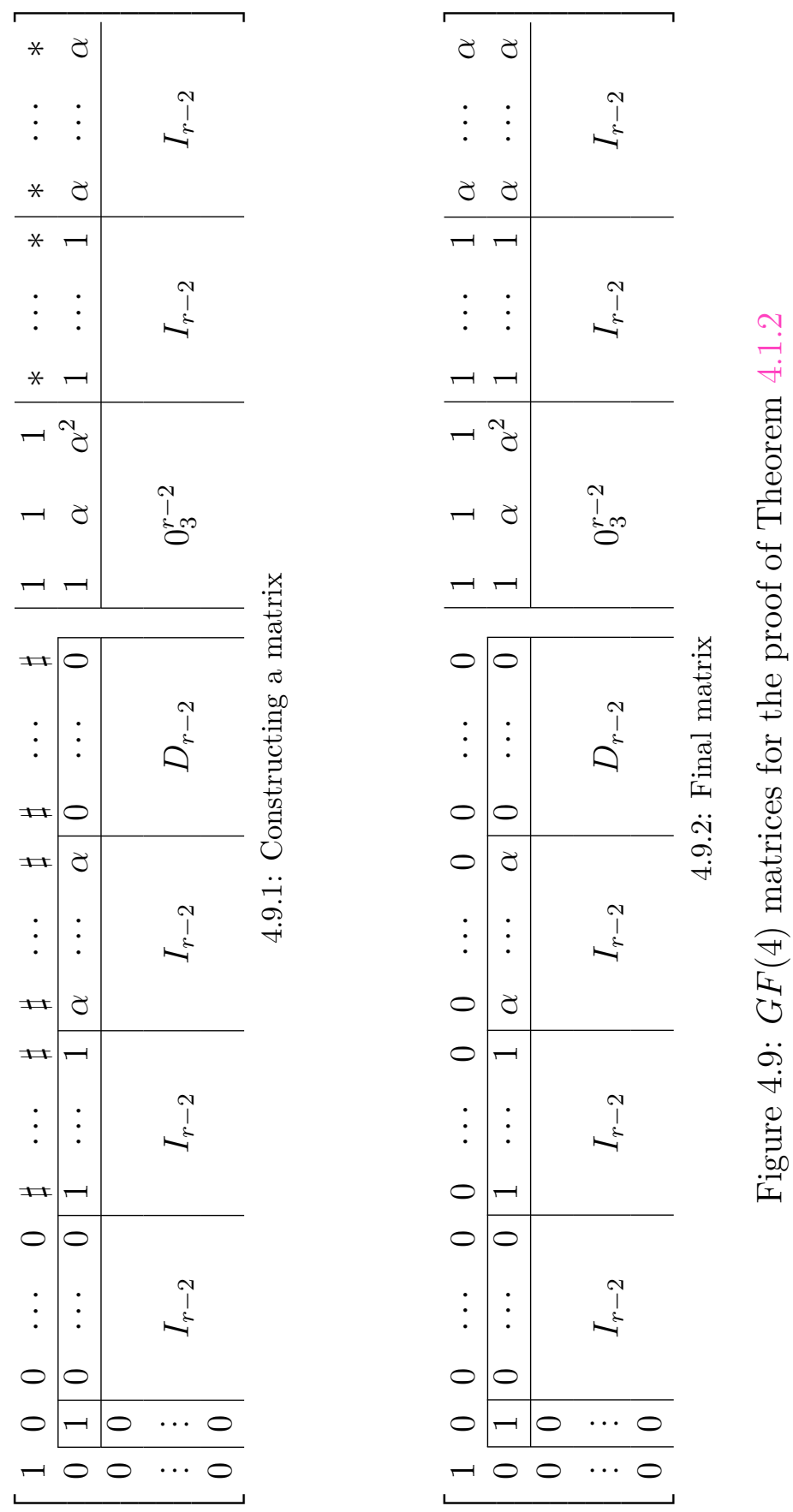
Since $f_{1}$ is on a five-point line with $e$, and $e$ is on a three-point line with all other $f_{i}$ s, we get that $M$ must be represented by a matrix shown in Figure 4.9.1, where each $\sharp$ is an element of $G F(4)$ and each $*$ is a non-zero element of $G F(4)$.

Now consider the matroid $\operatorname{si}\left(M / g_{r-2}\right)$. Upon considering the matrix of $M$, we see that $\epsilon(M)-\epsilon\left(M / g_{r-1}\right) \leq r+2$. However, we know that $\epsilon(M)-$ $\epsilon\left(M / g_{r-1}\right) \geq r+2$, and hence $\epsilon(M)-\epsilon\left(M / g_{r-1}\right)=r+2$, sosi $\left(M / g_{r-1}\right) \in \mathscr{G}_{r-1}$.

When we look at the matrix for $\operatorname{si}\left(M / g_{r-1}\right)$, we see a solitary five-point line and at least one four-point line. Hence $\operatorname{si}\left(M / g_{r-1}\right) \cong G_{r-1}$, and we are able to determine the values for $\sharp$. Also, we see that all except the last $*$ in each block are also fixed. To discover the last two values of $*$, we repeat this argument with $g_{r-2}$.

Hence $M$ must be represented by the matrix in Figure 4.9.2. When we take the $G F(4)$ matrix for $G_{r}$ given in Figure 2.5.2 and add the first row to the second row, we get the matrix for $M$ from Figure 4.9.2. Hence $M \cong G_{r}$.

However, this means that $M$ is not a counterexample to Theorem 4.1.2. Hence Theorem 4.1.2 is true.

\subsection{A Strategic Hurdle}

The proof of Theorem 1.1.17, which characterises the maximum-sized sixthroots-of-unity matroids, proceeds in much the same way that the results in this chapter go. Oxley, Vertigan, and Whittle [11] assume that $M / e$ is regular for some $e$, where $M$ is a maximum-sized sixth-roots-of-unity matroid, and $e$ is an element of $M$. Once they eliminate this case, they consider the case where $M / e$ is non-regular and use Lemma 2.4.2 to derive contradictions. These contradictions arise from placing a $U_{2,4}$-minor on two points of $\mathcal{L}(M, e)$, and then lifting $e$. They then show that this lift cannot be sixth-roots-ofunity, a contradiction.

In our proof, this final case proves troublesome. We have a 2-rounded set, $\left\{U_{2,5}, U_{3,5}, F_{7}^{=},\left(F_{7}^{=}\right)^{*}, Q_{6}\right\}$, by Lemma 2.4.3. However, it is possible to lift each member of this set while still remaining golden-mean. This was verified using the Sage code in Appendix 4.A.4. We then try to extend Defini- 
tion 2.4.1 to three element subsets of $E(M)$. However, any such "3-rounded" set must contain the matroid $\mathcal{W}_{3}^{+}$, which is the rank-three whirl with an extra point freely placed on any long line. We can also lift from this matroid in a golden-mean way, so a contradiction is not forthcoming.

\section{Appendix 4.A Code}

\section{A.1 Lemma 4.1.4}

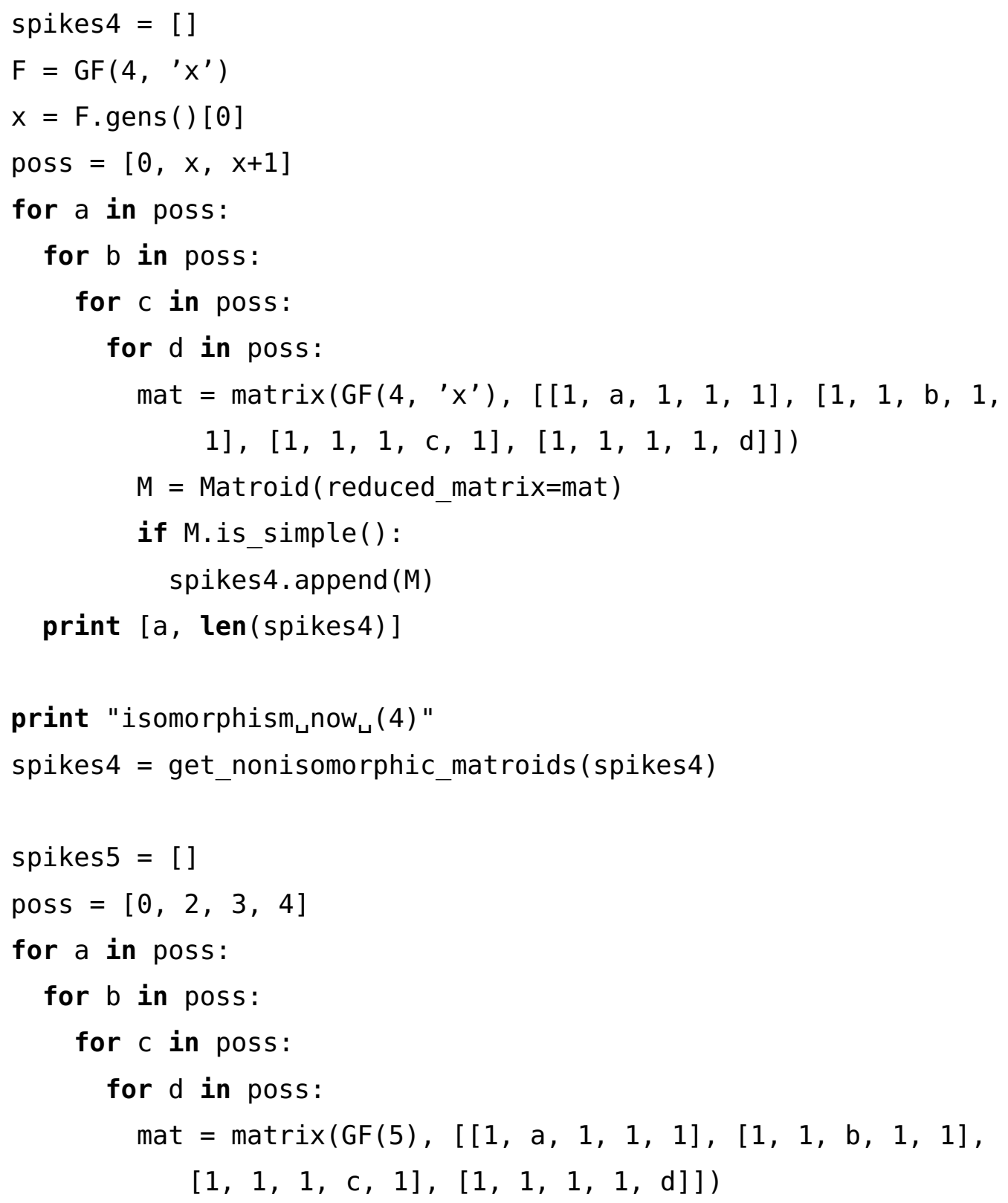




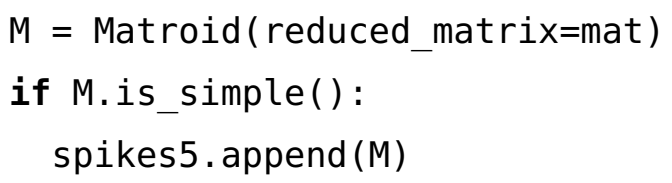

This code constructs a $G F(4)$-representation of all golden-mean 4-spikes. We know what the matrix for a 4-spike must look like, and we construct all possible 4-spikes over both $G F(4)$ and $G F(5)$. We then call the function matroid_set_intersection() from Appendix 2.A.2 to get the $G F(4) 4$ spikes that are isomorphic to a $G F(5)$ 4-spike, which results in the goldenmean 4-spikes by Theorem 1.2.2.

\section{A.2 Lemma 4.1 .5}

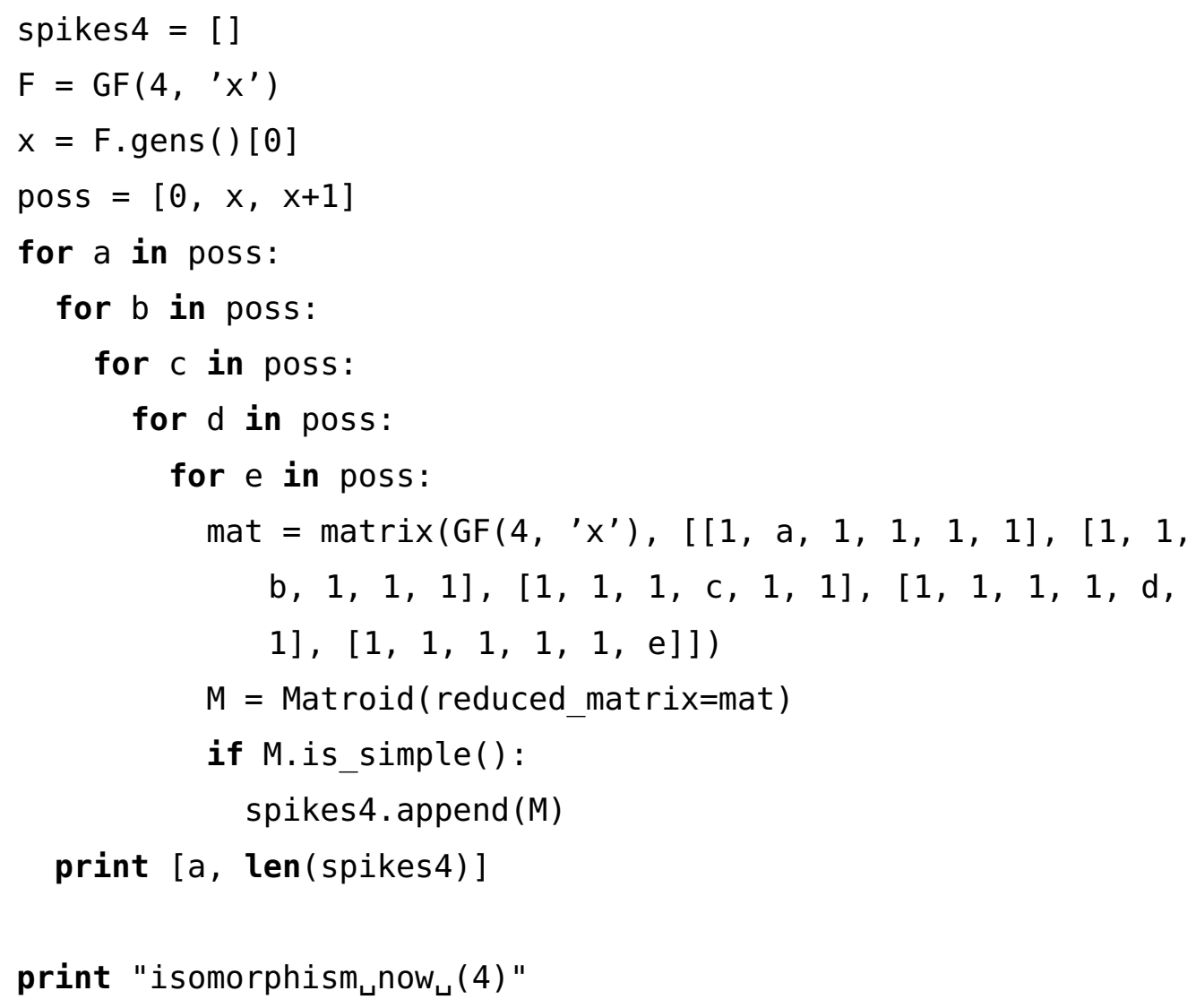




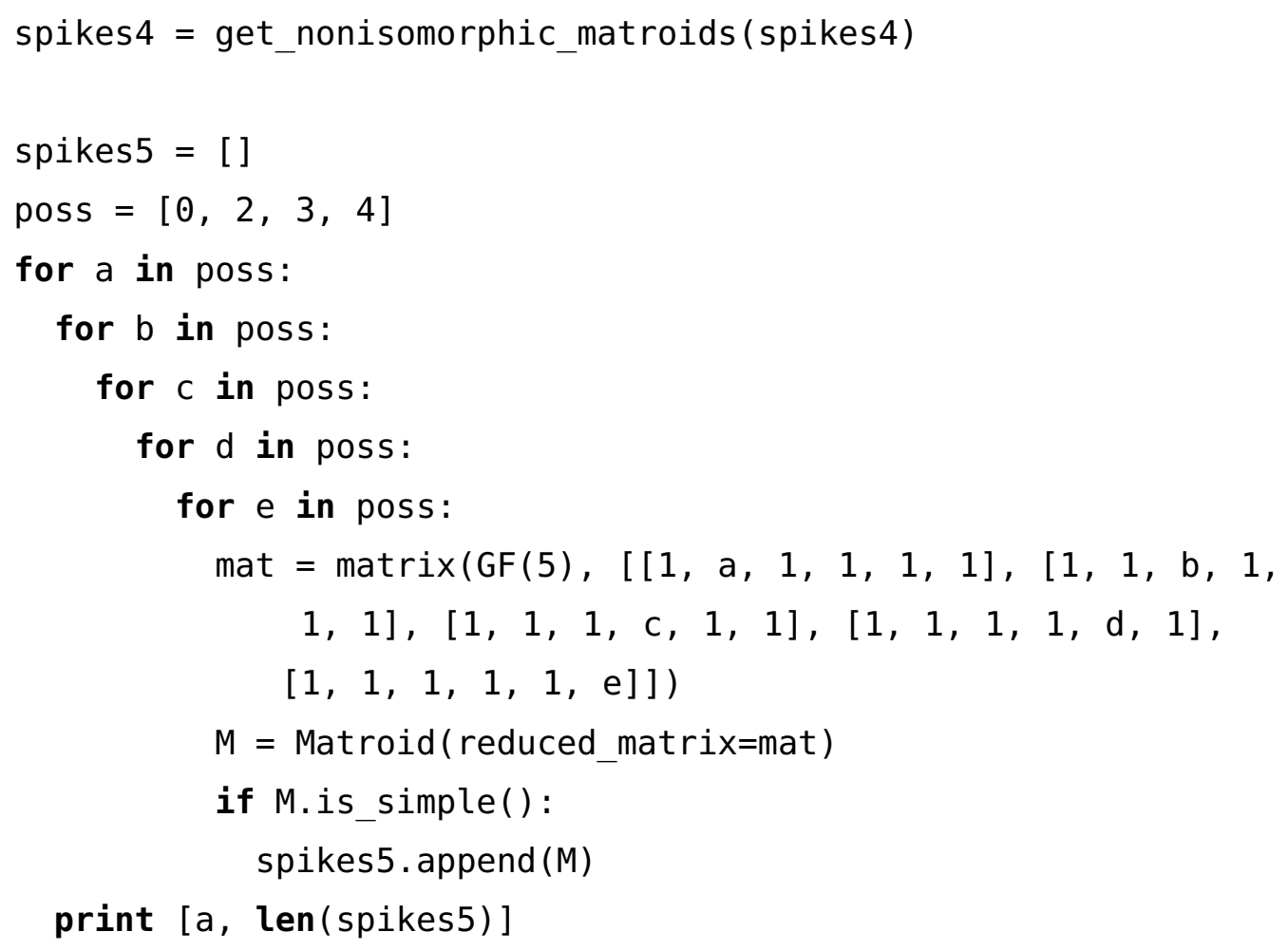

This code is almost identical to the code in Appendix 4.A.1. The difference is that it constructs 5 -spikes instead of 4 -spikes.

\section{A.3 Lemma 4.3.15}

\# Make $\mathrm{T}$ for comparison

TCC $=\{2:$ ['abdg', 'acfh', 'beh', 'def', 'ceg'], 3: ['abcdefgh' ]\}

$\mathrm{T}=$ Matroid(groundset='abcdefgh', circuit_closures $=\mathrm{TCC}$ )

start $=$ identity_matrix $(\mathrm{GF}(19), 3)$

$A=$ Matroid (matrix=start, groundset=' $a b c^{\prime}$ )

poss $=[]$

for B in A.linear_extensions(element=' $d$ ', fundamentals=funds, 
simple=True, $\left.F={ }^{\prime} a b^{\prime}\right)$ :

for $C$ in B.linear_extensions(element=' $e^{\prime}$, fundamentals=funds, simple=True, $\left.F=' a b^{\prime}\right)$ :

for $D$ in C.linear_extensions (element=' $f$ ', fundamentals $s$ funds, simple=True, $\left.F={ }^{\prime} a c^{\prime}\right)$ :

for $E$ in $D . l$ inear extensions (element $={ }^{\prime} g$ ', fundamentals= funds, simple=True, $\left.F={ }^{\prime} a c^{\prime}\right)$ :

for $F$ in E.linear_extensions(element $={ }^{\prime} h '$, fundamentals= funds, simple=True, $F={ }^{\prime}$ eg') :

if F.is_isomorphic $(T)$ :

poss.append (F)

for $H$ in poss:

for $I$ in $\mathrm{H}$.linear_coextensions(element=' $\mathrm{i}$ ', fundamentals= funds):

for $J$ in I.linear_extensions (element $={ }^{\prime} j{ }^{\prime}$, fundamentals= funds, simple=True, $F=' b i ')$ :

for $\mathrm{K}$ in $\mathrm{J}$.linear_extensions (element $={ }^{\prime} \mathrm{k}$ ', fundamentals= funds, simple=True, $F=' b i ')$ :

for $L$ in $K$.linear_extensions (element $={ }^{\prime} l '$ ', fundamental $s=$ funds, simple=True, $\left.F={ }^{\prime} a i^{\prime}\right)$ :

for $M$ in $L$.linear_extensions(element=' $\mathrm{m}$ ', fundamentals=funds, simple=True, $\left.F={ }^{\prime} a i^{\prime}\right)$ :

for $N$ in M.linear_extensions(element $=$ ' $n$ ', fundamentals=funds, simple=True, $\left.F=f^{\prime} i^{\prime}\right)$ :

for 0 in $\mathrm{N}$.linear_extensions(element $={ }^{\prime} \mathrm{O}$ ', fundamentals=funds, simple=True, $\left.F={ }^{\prime} f i^{\prime}\right)$ :

for $P$ in 0. linear_extensions(element $=$ ' $p$ ', fundamentals=funds, simple=True, $\left.F={ }^{\prime} g i^{\prime}\right)$ :

$P$

for $H$ in poss:

for $\mathrm{I}$ in $\mathrm{H}$. linear_coextensions (element=' $\mathrm{i}$ ', fundamentals= funds):

for $\mathrm{J}$ in $\mathrm{I}$. linear_extensions $\left(\right.$ element $={ }^{\prime} \mathrm{j}^{\prime}$, fundamentals= 


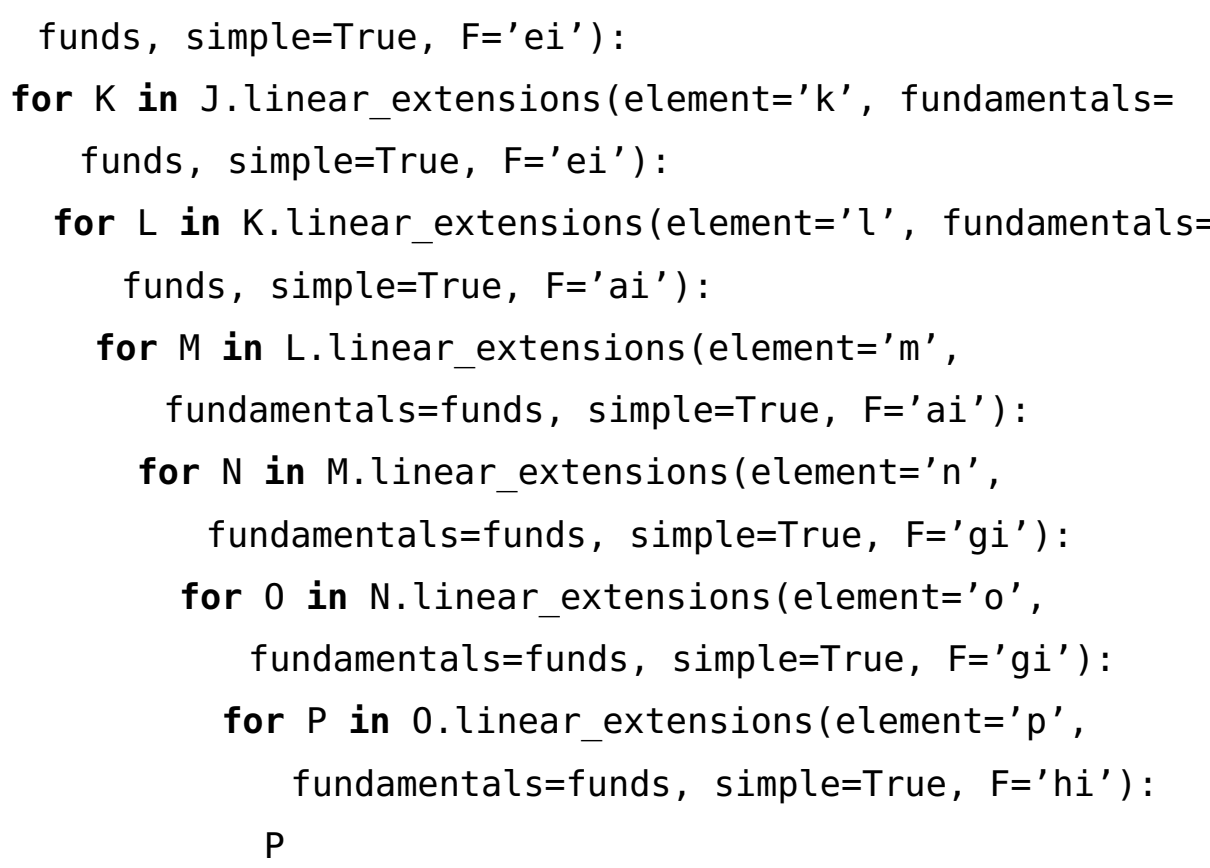

In the proof of Lemma 4.3.15, we come across a case where a counterexample to the lemma must have one of two very specific structures, detailed in cases (ii) and (iii) of Lemma 4.3.14. This code starts by constructing all possible golden-mean representations (over $G F(19)$ ) for $T_{3}^{1}$, and stores these in poss. We then attempt to construct the counterexamples to Lemma 4.3.15, by creating a lift of $T_{3}^{1}$, and extending into various lines. If any such matroids exist, we then print them. Upon running this code, nothing is output, so no counterexamples to Lemma 4.3.15 (in this particular case) exist.

\section{A.4 Section 4.4}

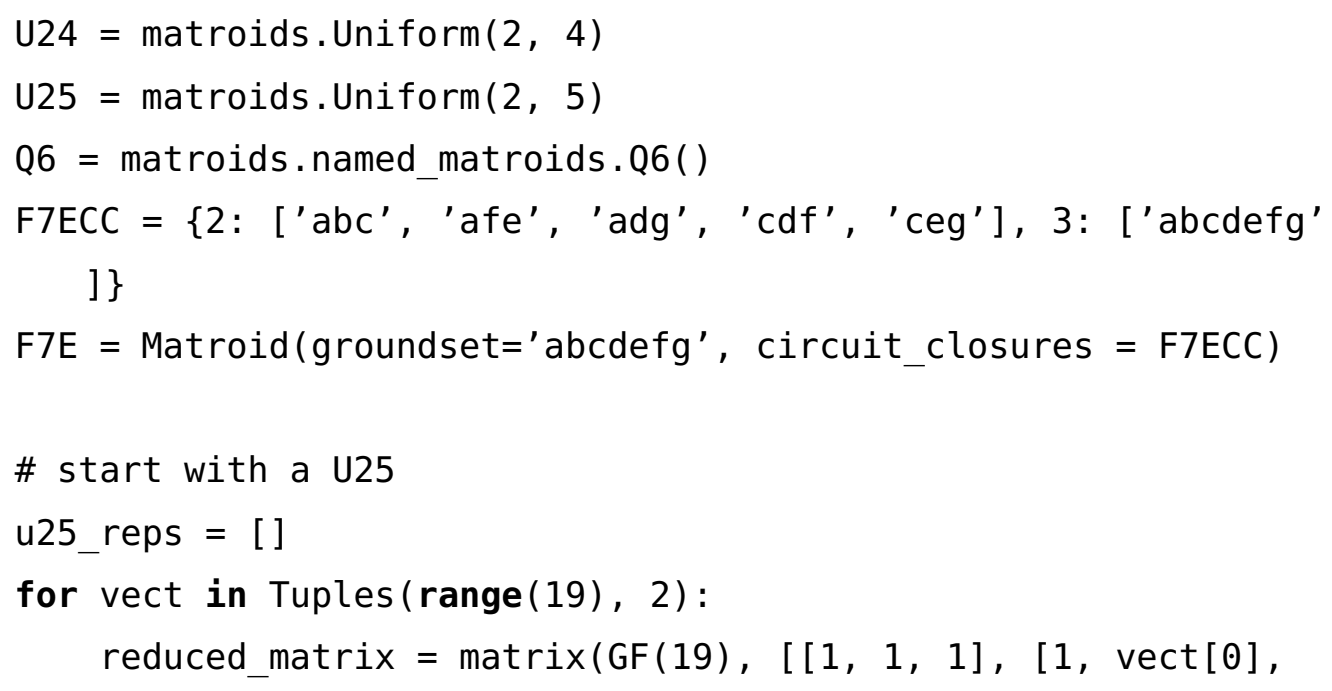




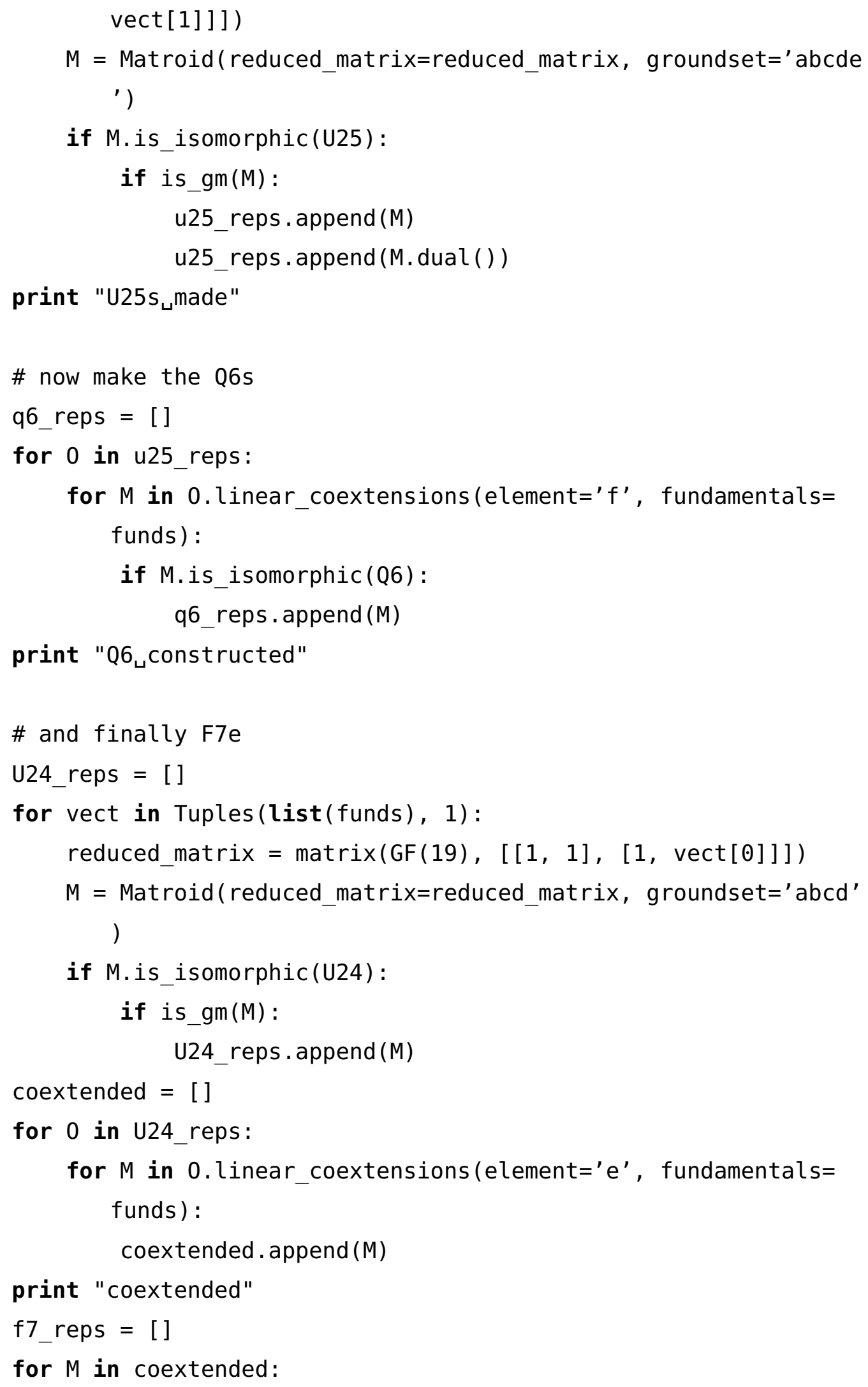


for $N$ in $M$. linear_extensions (element $={ }^{\prime} f^{\prime}$, simple=True, fundamentals=funds, $F={ }^{\prime}$ ae' ) :

for 0 in N.linear_extensions(element $t={ }^{\prime} g$ ', simple=True, fundamentals=funds, $\left.F={ }^{\prime} \mathrm{ad}^{\prime}\right)$ :

if 0. is_isomorphic(F7E) :

f7_reps.append ( 0$)$

f7_reps.append ( 0 .dual ( ))

print " $F 7 E_{\sqcup}$ constructed"

\# now lift

lifted $=[]$

for $M$ in 425 reps:

for $N$ in $M$.linear_coextensions(element=' $f$ ', fundamentals= funds) :

lifted. append ( $\mathrm{N})$

print "lifted (U25)"

for $M$ in $q 6$ reps:

for $N$ in $M$.linear_coextensions(element=' $g$ ', fundamentals= funds) :

lifted.append ( $N)$

print "lifted $(06)$ "

for $M$ in $f 7$ reps:

for $N$ in $M$. linear_coextensions $\left(\right.$ element $={ }^{\prime} h '$, fundamentals= funds):

lifted. append ( $\mathrm{N})$

print "lifted $(F 7=)$ "

baddies $=[]$

for $M$ in lifted:

gs $=$ M.groundset_list ()

long $=$ len (gs)

end $=$ gs [long -1$]$

for $x y$ in Combinations (range(long - 1), 2):

for $\mathrm{N}$ in $\mathrm{M}$.linear_extensions (simple=True, fundamentals= funds, $F=[$ end, gs $[x y[0]]])$ : 


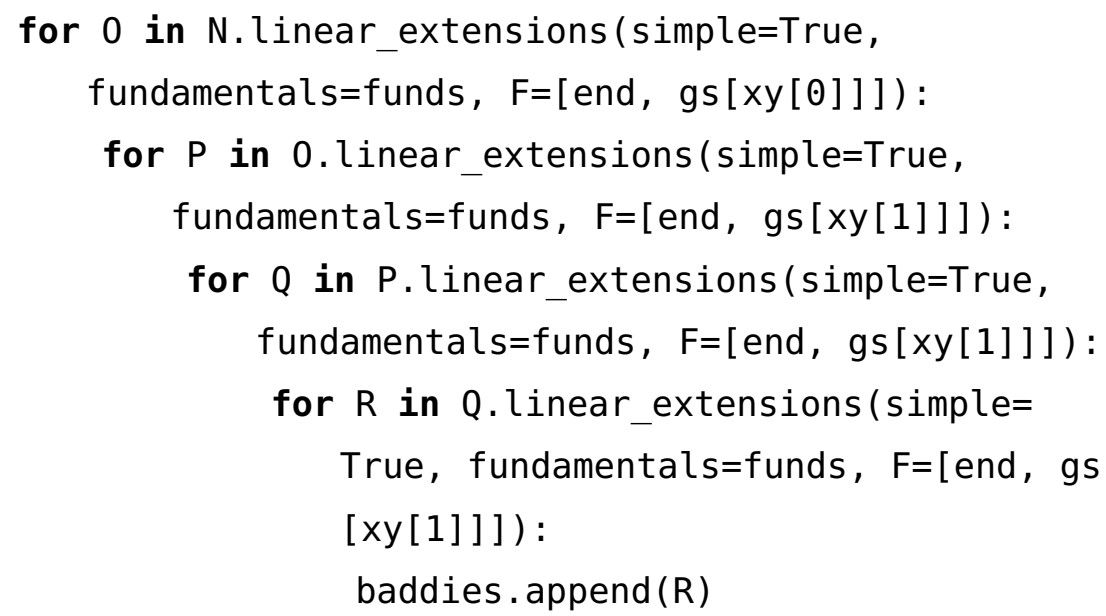

This code takes a matroid $\mathbf{M}$ from our 2-rounded set $\left\{U_{2,5}, U_{3,5}, F_{7}^{=},\left(F_{7}^{=}\right)^{*}, Q_{6}\right\}$, and coextends it freely by the element $e$ (stored in end). We then choose two points $x$ and $y$ of $\mathbf{M}$ (stored in $\mathrm{xy}$ ), and extend the line $\{x, e\}$ to a four-point line and the line $\{y, e\}$ to a five-point line. This produces two matroids (up to isomorphism), which means that a contradiction is not forthcoming. 
My Panasonic typewriter can make graphs. It types in four different colours.

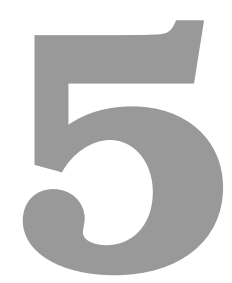

\section{G-graphic Matroids}

\section{$5.1 \quad$ Preliminaries}

Definition 5.1.1 (Van Zwam [20, Definitions 3.2.1 and 3.2.2]). A matroid $M$ is $\mathbb{P}$-graphic for some partial field $\mathbb{P}$ if there exists a $\mathbb{P}$-matrix $A$ with at most two non-zero entries per column such that $M$ is represented over $\mathbb{P}$ by $A$.

In this chapter, we prove the following theorem.

Theorem 5.1.2. Let $\mathcal{T}$ be the class of golden-mean-graphic matroids. Then

$$
h_{\mathcal{T}}(r)=\left(\begin{array}{c}
r+3 \\
2
\end{array}\right)-5 \text {. }
$$

Furthermore, $M \in \mathcal{T}$ is maximum-sized if and only if $M$ is isomorphic to either $T_{r(M)}^{2}$ or $G_{r(M)}$.

Let $\mathfrak{M}$ be a $\mathbb{G}$-matrix representing a loopless matroid $M$ with at most two non-zero entries per column. From $\mathfrak{M}$ we are able to construct a directed 

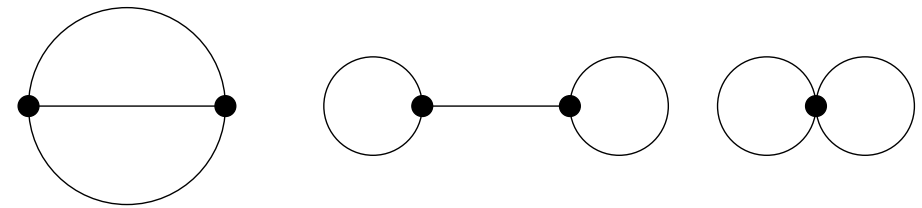

Figure 5.1: From left to right: the theta, barbell, and handcuff graphs

weighted graph $W$. Firstly, scale each column of $\mathfrak{M}$ so that the first nonzero entry is one. Each row of $\mathfrak{M}$ corresponds to a vertex, and each column corresponds to an edge. If a column of $\mathfrak{M}$ contains non-zero entries in rows $i$ and $j$, where row $i$ is above row $j$, then that column corresponds to a directed edge from $i$ to $j$, and the weight of this edge is the entry from row $j$. If a column of $\mathfrak{M}$ contains only one non-zero entry, in row $i$, then that column corresponds to a loop on $i$, weighted one.

Thus each edge in $W$ corresponds to an element of $M$. A cycle $C$ in $W$ is balanced if the product of its edge weights is equal to one. Let $c$ be an edge from $C$, with weight $w_{c}$. If $c$ is directed in accordance with the direction on $C$, then we use $w_{c}$ in our product. If $c$ is directed counter to the direction on $C$, then we use $w_{c}^{-1}$ in our product.

Lemma 5.1.3 (Zaslavsky [26, Matroid Theorem]). Let $M$ be a $\mathbb{P}$-graphic matroid with associated weighted directed graph $W$. Then the circuits of $M$ correspond to balanced cycles of $W$; or to theta, barbell, or handcuff graphs (see Figure 5.1) that contain no balanced cycles.

In order to prove Theorem 5.1.2, we require $\mathcal{T}$ to be minor-closed.

Lemma 5.1.4 (Van Zwam [20, Proposition 3.2.3]). Let $\mathbb{P}$ be a partial field. The class of $\mathbb{P}$-graphic matroids is minor-closed.

Lemma 5.1.5 (Zaslavsky [27]). Let $M$ be a $\mathbb{G}$-graphic matroid, and let $W$ be a weighted directed graph associated with $M$. Let $W^{\prime}$ be a minor of $W$. Then there is a minor $M^{\prime}$ of $M$ such that $M^{\prime}$ has a $\mathbb{G}$-graphic representation corresponding to $W^{\prime}$.

Let $M$ be a simple $\mathbb{G}$-graphic matroid, and let $W$ be a corresponding weighted directed graph. If $e$ is a non-loop edge in $W$, then $W / e$ is the corresponding graph for the $\mathbb{G}$-graphic matroid $M / e$. On the other hand, if $e$ is a loop of $W$ 


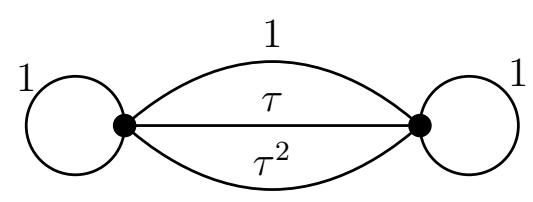

Figure 5.2: $\mathbb{G}$-graphic representation of $U_{2,5}$

at the vertex $v$, then we let $G^{\prime}$ be the graph obtained from $G$ by deleting $v$, and replacing non-loop edges $u_{i} v$ incident with $v$ by loops that are incident with $u_{i}$. Then $G^{\prime}$ corresponds to $M / e$, with loops deleted.

Example 5.1.6. The matroid $U_{2,5}$ has the $\mathbb{G}$-graphic representation shown in Figure 5.2, and is represented over $\mathbb{G}$ by the following matrix.

$$
\left[\begin{array}{ccccc}
1 & 0 & 1 & 1 & 1 \\
0 & 1 & 1 & \tau & \tau^{2}
\end{array}\right]
$$

\subsubsection{Graphs With No $K_{4}$}

Definition 5.1.7 (Diestel [3, Page 11]). Let $G$ be a connected graph. If $v$ is a vertex such that $G \backslash v$ is disconnected, then $v$ is a $\boldsymbol{c u t}$ vertex.

Definition 5.1.8 (Diestel [3, Page 60]). A block of $G$ is a maximal connected subgraph without a cut vertex.

Lemma 5.1.9 (Oxley [10, Lemma 5.4.11]). A simple 2-connected graph in which the degree of every vertex is at least three has a subgraph that is a subdivision of $K_{4}$.

The following result, which is related to series-parallel networks, is wellknown. A proof is given here for completeness.

Lemma 5.1.10. Let $G$ be a simple graph with no $K_{4}$-minor. Then $G$ has a vertex of degree at most two.

Proof. Assume for a contradiction that all vertices of $G$ have degree at least three. Without loss of generality, we can assume that $G$ is connected. Amongst connected counterexamples, assume that $G$ has been chosen so that it has the smallest possible number of edges. 
Assume that $G$ is 2-connected. Then $G$ is a simple 2-connected graph in which the degree of every vertex is at least three, so Lemma 5.1.9 applies, and we deduce that $G$ has a $K_{4}$-minor. However, this is a contradiction to the definition of $G$, so $G$ cannot be 2-connected.

Hence $G$ is connected but not 2-connected, and must have at least two blocks. An end-block is a block of $G$ that is incident with only one cut-vertex. It is well-known (see [3, Lemma 3.1.4] for example) that every graph that is connected but not 2-connected must have at least two end-blocks. Consider two of the end-blocks, $B_{1}$ and $B_{2}$, of $G$. Let $v_{i}$ be the cut vertex between $B_{i}$ and $G \backslash B_{i}$, for $i \in\{1,2\}$.

Since $B_{1}$ and $B_{2}$ have fewer edges than $G$, they each have at least one vertex of degree at most two. In fact, the only vertex in $B_{i}$ of degree at most two is $v_{i}$, for otherwise, the vertex with degree at most two in $B_{i}$ would also be in $G$. If $v_{i}$ has degree one in $B_{i}$, then since $B_{i}$ is a block, it follows that $B_{i}$ contains a single edge, and hence $G$ contains a vertex of degree one. Therefore $v_{i}$ has degree exactly two in $B_{i}$.

Now let $G^{\prime}$ be the graph obtained from $B_{1}$ and $B_{2}$ by identifying them at the vertices $v_{1}$ and $v_{2}$. Our previous paragraph shows that $G^{\prime}$ has no vertices of degree at most two. As $G^{\prime}$ is a minor of $G$, it must be equal to $G$, or else the minimality of $G$ is contradicted. Let $c$ be the unique cut-vertex in $G$. Note that $c$ has degree four.

Let $u_{i}$ and $w_{i}$ be the two vertices incident with $c$ in $B_{i}$, for $i \in\{1,2\}$. Consider the graph $G^{\prime \prime}$, where $V\left(G^{\prime \prime}\right)=V(G) \backslash\{c\}$ and $E\left(G^{\prime \prime}\right)=E(G) \cup\left\{u_{1} u_{2}, w_{1} w_{2}\right\}$. $G^{\prime \prime}$ cannot have a $K_{4}$-minor as neither $B_{1}$ nor $B_{2}$ has a $K_{4}$-minor, and, as $K_{4}$ has no 2-separation, any $K_{4}$-minor of $G^{\prime \prime}$ would use at most one vertex of either $B_{1}$ or $B_{2}$, which would imply that $B_{1}$ or $B_{2}$ has a $K_{4}$-minor, a contradiction. As $|E(G)|-\left|E\left(G^{\prime \prime}\right)\right|=2$, the minimality of $G$ implies that $G^{\prime \prime}$ has a vertex of degree at most two. This vertex must have the same degree in $G$, hence $G$ must have a vertex of degree at most two. 


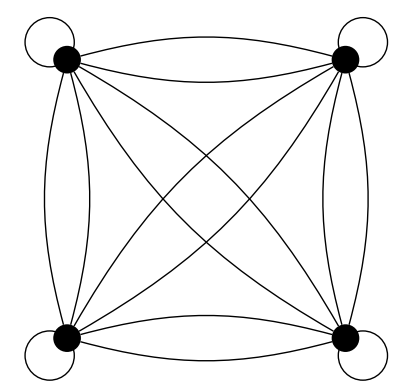

Figure 5.3: The graph $2 K_{4}^{\circ}$

\subsection{Results}

Lemma 5.2.1. Let $M$ be a maximum-sized $\mathbb{G}$-graphic matroid. Let $W$ be a weighted directed graph that corresponds to a representation of $M$. Then $W$ has a loop at every vertex.

Proof. Let $\mathfrak{M}$ be a $\mathbb{G}$-matrix representing $M$ such that each column of $\mathfrak{M}$ has at most two non-zero entries and $\mathfrak{M}$ corresponds to $W$. If $W$ does not have a loop at every vertex, then $\mathfrak{M}$ does not contain every standard basis vector. Adding standard basis vectors as columns to $\mathfrak{M}$ does not change its sub-determinants, and thus we are able to do so, contradicting the fact that $M$ is maximum-sized.

Definition 5.2.2. The graph $2 K_{4}^{\circ}$ is the graph $K_{4}$ with every edge being a parallel class of size two, and a single loop at every vertex. This is shown in Figure 5.3.

Lemma 5.2.3. Let $W$ be a weighted directed graph corresponding to a $\mathbb{G}$ graphic matroid. Then $W$ has no $2 K_{4}^{\circ}$-minor.

Proof. We prove this using Sage [19]. The code is in Appendix 5.A.1. The algorithm we use constructs a representation for $2 K_{4}^{\circ}$ over $G F(19)$ and fills it out using elements of the set $\{1, \ldots, 19\}$, and then tests for $\mathbb{G}$-representability using the function is_gm() from Appendix 2.A.2. As no results are given, this shows that $2 K_{4}^{\circ}$ is not a $\mathbb{G}$-graphic matroid, and hence $W$ cannot have a $2 K_{4}^{\circ}$-minor by Lemma 5.1.5.

Lemma 5.2.4. Let $M$ be a non-ternary $\mathbb{G}$-graphic matroid, and let $W$ be a weighted directed graph that corresponds to a representation of $M$. Let e and $f$ be loops of $W$. Then $M$ has a minor $M^{\prime}$ where 
(i) $W^{\prime}$ is a weighted directed graph that corresponds to a representation of $M^{\prime}$

(ii) $W^{\prime}$ is a minor of $W$,

(iii) $e$ and $f$ are loops in $W^{\prime}$, and

(iv) $M^{\prime}$ is isomorphic to $U_{2,5}$.

Proof. As $M$ is non-ternary, it must have one of the excluded minors for $G F(3)$-representability (see [10, Theorem 10.2.1]) as a minor. Because neither $F_{7}$ nor $F_{7}^{*}$ is $G F(5)$-representable, we see that $M$ must have either $U_{2,5}$ or $U_{3,5}$ as a minor.

Hence we can apply Lemma 2.4.3, so $M$ has a minor $N$ isomorphic to a member of $\left\{U_{2,5}, U_{3,5}, F_{7}^{=},\left(F_{7}^{=}\right)^{*}, Q_{6}\right\}$ that uses $e$ and $f$. Now conditions (i) and (ii) hold by Lemmas 5.1.4 and 5.1.5. Condition (iii) holds as loops of $N$, where $N \leq M$, are loops of $M$.

From Example 5.1.6, we know that it is possible for $N$ to be isomorphic to $U_{2,5}$. In this case, we take $M^{\prime}$ to be $N$ and we are done.

We now show that $N$ cannot be isomorphic to $U_{3,5}, F_{7}^{=}$, or $\left(F_{7}^{=}\right)^{*}$.

Sublemma 5.2.4.1. It is not possible for $N$ to be isomorphic to $U_{3,5}, F_{7}^{=}$, or $\left(F_{7}^{=}\right)^{*}$.

Subproof. We prove this by attempting to construct a $G F(4)$-graphic representation for each matroid, and failing in each case. The code used is in Appendix 5.A.2.

Hence $N$ must be isomorphic $Q_{6}$. We now show that we are able to find $M^{\prime}$ in this case.

Sublemma 5.2.4.2. If $N$ is isomorphic to $Q_{6}$, then there is an element $x$ of $Q_{6}$, such that $x$ does not correspond to a loop in the weighted graph that corresponds to $N$, and such that $N / x$ is isomorphic to $U_{2,5}$.

Subproof. We prove this by constructing all $G F(4)$-graphic representations for $Q_{6}$ with at least two loops. We then contract a non-loop element, and discover a matroid isomorphic to $U_{2,5}$. The code is in Appendix 5.A.3.

Hence we can always find $M^{\prime}$, as desired. 


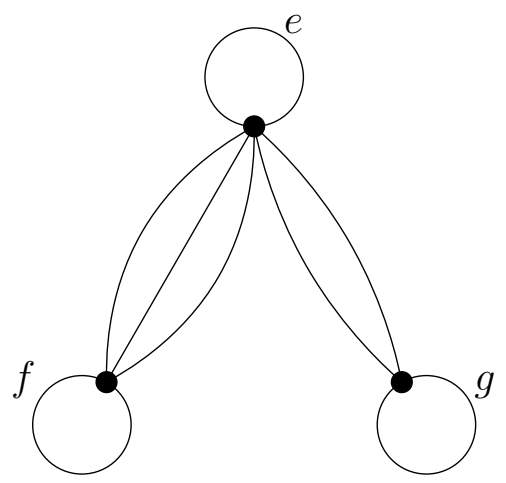

Figure 5.4: Labels for Lemma 5.2.5

Lemma 5.2.5. Let $M$ be a counterexample to Theorem 5.1 .2 of minimum rank, and assume that $M$ is maximum-sized. Then a four-point line cannot meet a five-point line.

Proof. Assume for a contradiction that the lemma is false. In $W$, the weighted directed graph associated with $M$, the five-point line must correspond to two loops, and three edges joining them, and the four-point line must correspond to two loops with two edges joining them. If $e$ is the element in both lines, then $e$ is a loop. Let $f$ and $g$ be the other two loops, as shown in Figure 5.4.

Consider the matroid $\operatorname{si}(M / e)$. We can assume that $\operatorname{si}(M / e)$ is non-ternary as, if it were ternary, then we could apply Theorem 4.1.2. Let $Z$ be a subset of $E(M)$ such that $M / e \backslash Z \cong \operatorname{si}(M / e)$. We can assume that neither $f$ nor $g$ is in $Z$. Then, by Lemma 5.2.4, $M / e \backslash Z$ has a $U_{2,5}$-minor $N$ using $f$ and $g$. Let $I$ and $J$ be disjoint subsets of $E(M / e \backslash Z)$ such that $M / e \backslash Z / I \backslash J=N$. Now, the matroid $M / I \backslash J$ is a rank-three matroid with eleven points, and hence is isomorphic to $B R$ by Lemma 2.3.1. However, $B R$ does not contain a five-point line, so we have arrived at a contradiction.

Lemma 5.2.6. Let $M$ be a rank-r counterexample to Theorem 5.1 .2 of minimum rank, and assume that $M$ is maximum-sized. Then there are no fivepoint lines in $M$.

Proof. Assume there is a five-point line $\ell$ in $M$. The line $\ell$ must have two loops in $W$, the weighted directed graph associated with $M$, labelled $f$ and $g$. Since $r(M) \geq 4$, there has to be a non-loop edge $e$ incident with $f$ or 


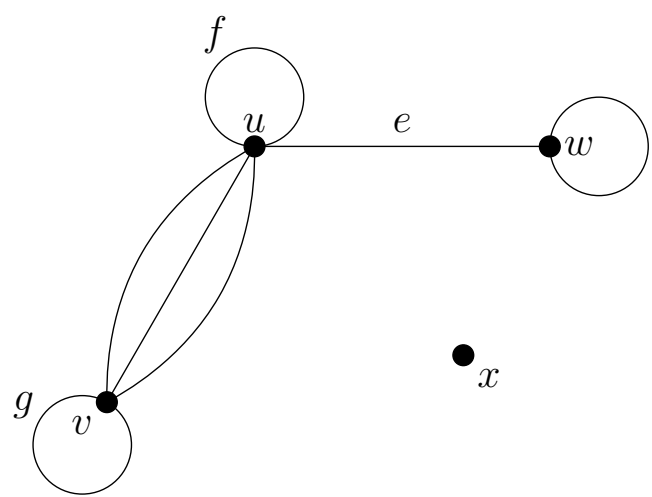

Figure 5.5: Labels for Lemma 5.2.6

$g$, or else $W$ is not connected, implying that $M$ is not connected. Without loss of generality, we can assume that $e$ is incident with $f$. This is shown in Figure 5.5.

Now consider $\epsilon(M)-\epsilon(M / e)$. First, $e$ is not in a four or five-point line, since that line would have to contain $f$, contradicting Lemma 5.2.5 or Corollary 4.2.7. There is one barbell (Figure 5.1) containing $e$, and every other three-point line containing $e$ corresponds to a balanced triangle in $W$. We claim that there is at most one such triangle incident with every vertex other than the end-vertices of $e$. Using the labels from Figure 5.5, note that there is at most one edge between $v$ and $w$, since otherwise $g$ is in a four-point line because every vertex is incident with a loop by Lemma 5.2.1. Therefore we can see that $e$ is in at most one balanced triangle using the vertex $w$. If $x$ is a vertex of $W$ that is not $u, v$, or $w$, then there is at most one edge between $u$ and $x$, for the same reason, and therefore $e$ is in at most one balanced triangle incident with $x$. Now $e$ is in no $U_{2,4}$-restriction, and in at most $r-1$ three-point lines, so $\epsilon(M)-\epsilon(M / e)$ is at most $r$.

However, as $M$ is a counterexample to Theorem 5.1.2, it must be that $\epsilon(M) \geq$ $\left(\begin{array}{c}r+3 \\ 2\end{array}\right)-5$. Furthermore, the minimality of $M$ implies that $\epsilon(M / e) \leq\left(\begin{array}{c}r+2 \\ 2\end{array}\right)-5$. Hence $\epsilon(M)-\epsilon(M / e)$ is at least $r+2$.

We now have a contradiction, and hence there can be no five-point lines in $M$.

Let $X$ be the subgraph induced by removing all loops and one edge from every parallel class in $W$. Observe that there is an edge between $u$ and $v$ in $X$ if and only if there are multiple edges between $u$ and $v$ in $W$. 
Corollary 5.2.7. Let $M$ be a counterexample to Theorem 5.1.2 of minimum rank, and assume that $M$ is maximum-sized. Then $X$, as constructed above, has no $K_{4}$-minor.

Proof. If $X$ has a $K_{4}$-minor, then $W$, the weighted directed graph associated with $M$, has a $2 K_{4}^{\circ}$-minor, which contradicts Lemma 5.2.3.

Corollary 5.2.8. Let $M$ be a counterexample to Theorem 5.1.2 of minimum rank, and assume that $M$ is maximum-sized. Then $X$, as constructed above, has a vertex of degree two.

Proof. As Corollary 5.2.7 shows, $X$ has no $K_{4}$-minor, and hence Lemma 5.1.10 applies and so $X$ has a vertex of degree two.

Lemma 5.2.9. Let $M$ be a rank-r counterexample to Theorem 5.1.2 of minimum rank, and assume that $M$ is maximum-sized. Let $W$ be the weighted directed graph associated with $M$, and let e be a loop in $W$ that is incident with the vertex discovered in Corollary 5.2.8. Then $\mathrm{si}(M / e)$ is isomorphic to either $T_{r-1}^{2}$ or $G_{r-1}$.

Proof. Construct $X$ as described above, and apply Corollary 5.2.8 to find $e$. Note that edges in $X$ correspond to very long lines in $M$. As there are no five-point lines in $M$ by Lemma 5.2.6, each edge in $X$ corresponds to a four-point line in $X$. Hence $e$ can be on at most two four-point lines in $M$. Therefore, $e$ is on at most two four-point lines and $r-3$ three-point lines. Let $Z$ be a subset of $E(M)$ such that $M / e \backslash Z \cong \operatorname{si}(M / e)$. $Z$ can have at most one element from each three-point line and two elements from each four-point line. Thus the maximum value of $|Z|$ is $(r-3)+(2 \times 2)=r+1$. So

$$
\epsilon(M)-\epsilon(M / e)=|Z|+1 \leq r+2 .
$$

Furthermore, since $M$ is a minimal counterexample, we have that $\epsilon(M) \geq$ $\left(\begin{array}{c}r+3 \\ 2\end{array}\right)-5$, and that $\epsilon(M / e) \leq\left(\begin{array}{c}r+2 \\ 3\end{array}\right)-5$. Hence

$$
\epsilon(M)-\epsilon(M / e) \geq r+2
$$

When we combine (5.2.1) with (5.2.2), we see that all inequalities in those two equations are equalities. Hence $\operatorname{si}(M / e)$ is the same size as a maximum- 
sized golden-mean matroid, and the result follows from the minimality of $M$.

We are now able to prove Theorem 5.1.2. Let $M$ be a rank- $r$ counterexample to Theorem 5.1.2 of minimum rank, and assume that $M$ is maximum-sized. Let $W$ be the weighted directed graph associated with $M$, and let $e$ be the loop of $W$ contracted in Lemma 5.2.9.

Consider si $(M / e)$. By Lemma 5.2.9, when we construct si $(M / e)$ we get either $G_{r-1}$ or $T_{r-1}^{2}$, both of which contain a five-point line. However, as $e$ is a loop, it is not possible for $\mathrm{si}(M / e)$ to have five-point lines that are not present in $M$. Hence $M$ has a five-point line, which is a contradiction to Lemma 5.2.6. Therefore there can be no counterexamples to Theorem 5.1.2, and so the theorem is true, as desired.

\section{Appendix 5.A Code}

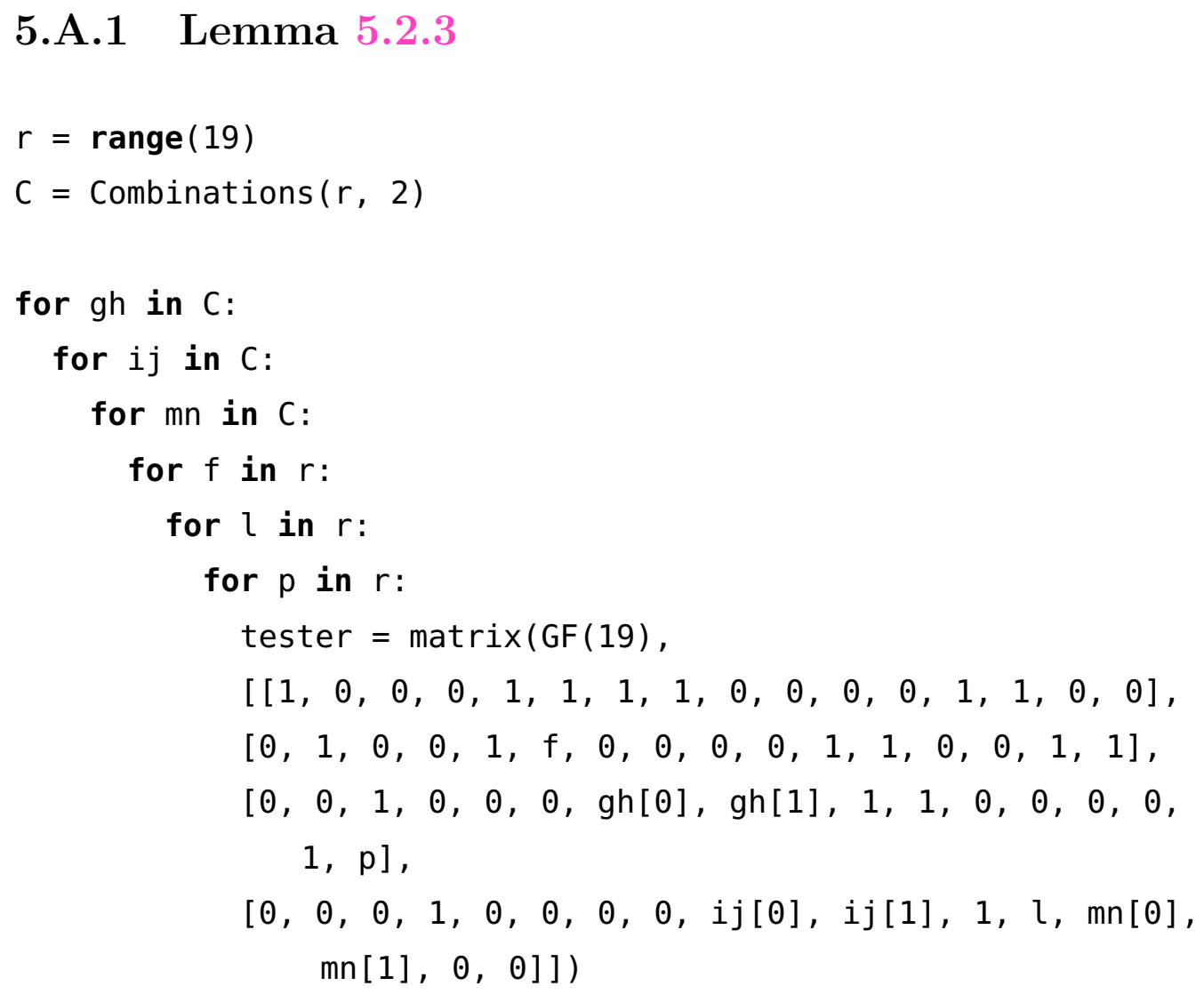




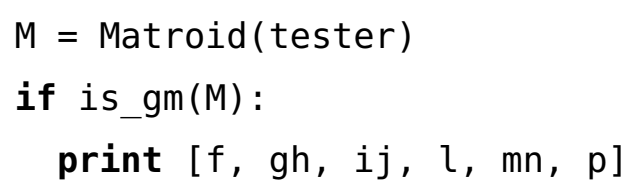

This code, which takes a long time, tests the graph $2 K_{4}^{\circ}$ to see if it could be a directed weighted graph corresponding to a $\mathbb{G}$-graphic matroid. As nothing is output, there is no $\mathbb{G}$-graphic matroid with corresponding directed weighted graph isomorphic to $2 K_{4}^{\circ}$.

\section{A.2 Sublemma 5.2.4.1}

\section{A.2.1 $U_{3,5}$}

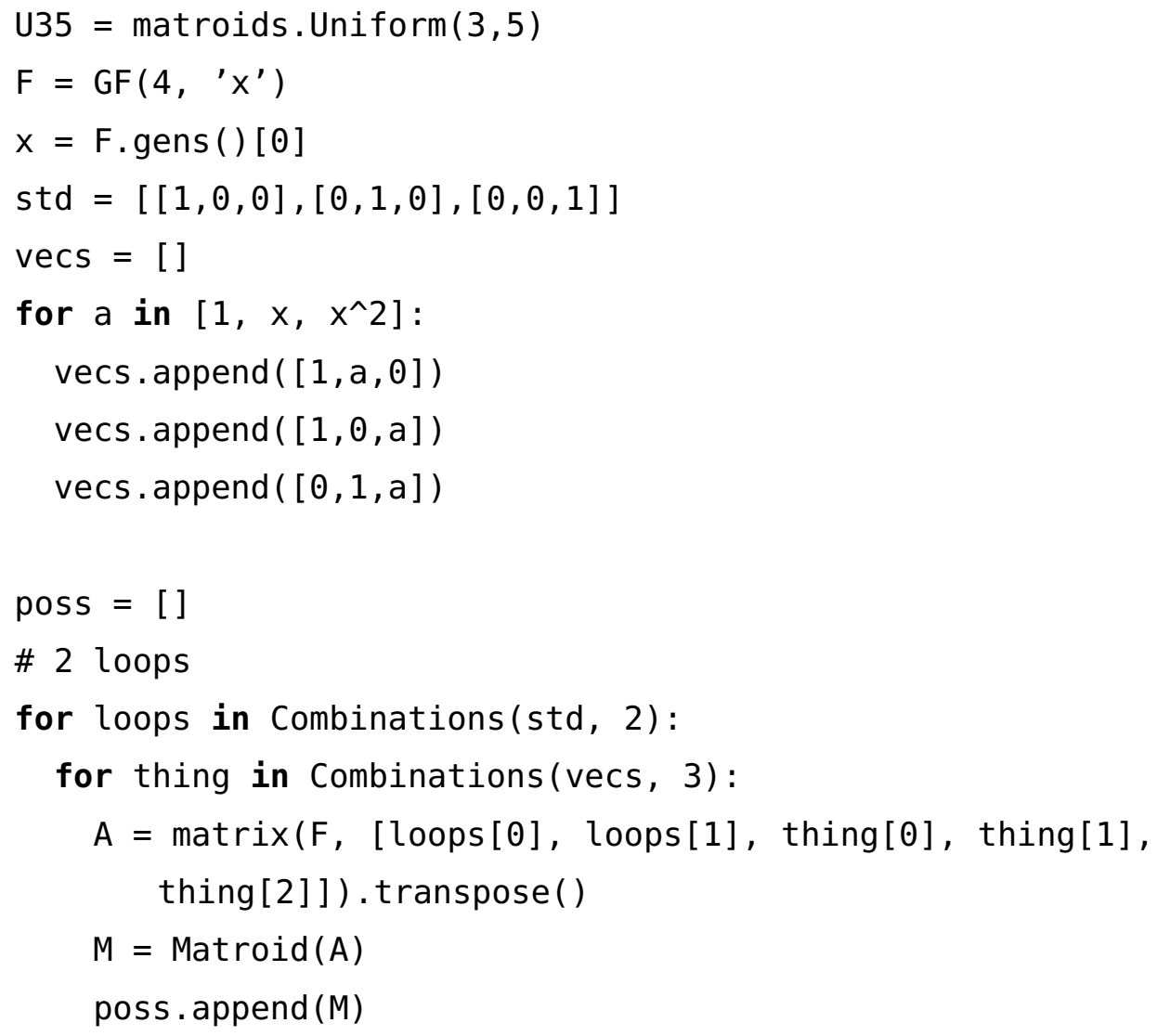


poss.append (M)

any([M.is_isomorphic(F7E) for $M$ in poss])

This code creates all simple rank-three seven-element $G F(4)$-graphic matroids with at least two loops and stores them in poss. It then checks to see if any members of poss are isomorphic to $F_{7}^{=}$, and outputs False, so no members of poss are isomorphic to $F_{7}^{=}$.

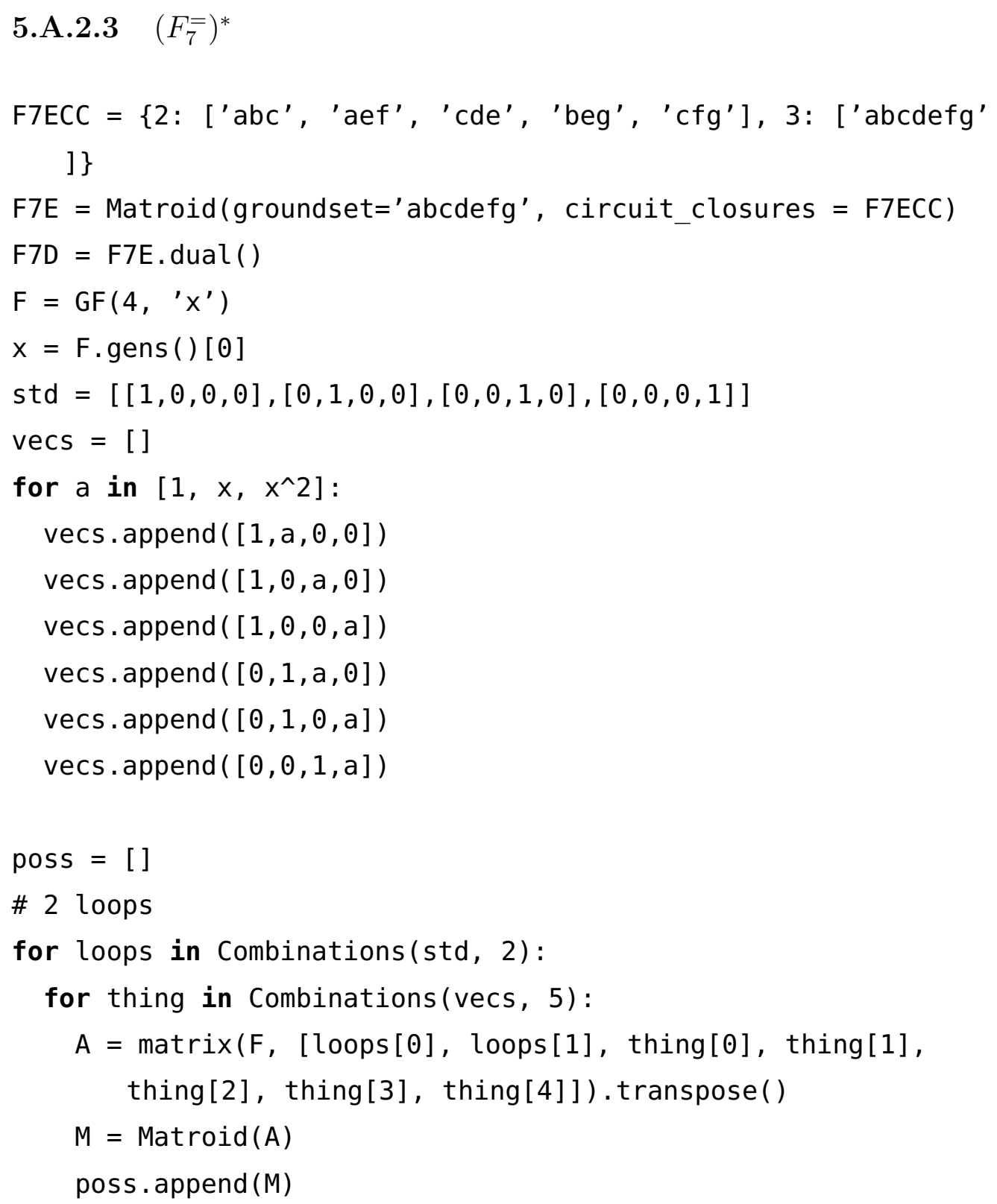


...the handyman's secret weapon, duct tape.

Red Green

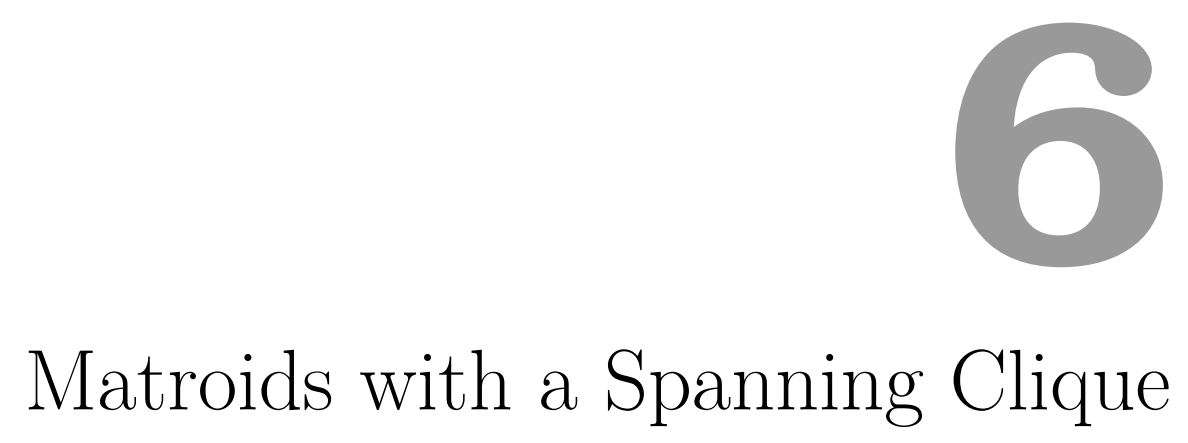

Warning: In this chapter, we make heavy use of colour.

\subsection{Preliminaries}

We start with some definitions that will be used throughout this chapter.

Definition 6.1.1. Let $M$ be a rank-r matroid with a $M\left(K_{r+1}\right)$-restriction. We call this restriction a spanning clique for $M$.

Definition 6.1.2. Let $M$ be a matroid with a spanning clique $N$. A nonclique line $l$ is a line of $M$ such that there exists an $e \in l$ such that $\{e\} \cap E(N)=\varnothing$.

Definition 6.1.3. Let $G$ be a graph. A star in $G$ is a $K_{1, n}$ subgraph of $G$, where $n \geq 2$. The vertex of the star that all edges of the star are incident with is called the core of the star.

In this chapter, we prove the following theorem. This proves Conjecture 1.2.3 in the class of golden-mean matroids with a spanning clique. 
Theorem 6.1.4. Let $\mathcal{M}$ be the set of golden-mean matroids that have a spanning clique. Let $\mathcal{C}$ be the family of minors of matroids in $\mathcal{M}$. Then

$$
h_{\mathfrak{C}}(r)= \begin{cases}\left(\begin{array}{c}
r+3 \\
2
\end{array}\right)-5 & \text { if } r \neq 3 \\
11 & \text { if } r=3 .\end{cases}
$$

Furthermore, $M \in \mathrm{C}$ is maximum-sized if and only if $M$ is isomorphic to a member of $\mathscr{G}_{r(M)}$ when $r(M) \neq 3$, or $M$ is isomorphic to the Betsy Ross when $r(M)=3$.

In Section 6.7, we outline how we anticipate that recent work by Geelen and Nelson (personal correspondence), when combined with Theorem 6.1.4, will lead to a proof of Conjecture 1.2.3 for matroids with sufficiently large rank. In order to prove this theorem, we need to know that it is true for ranks three and four. Rank-three follows from Lemma 2.3.1. For rank four, we have the following lemma.

Lemma 6.1.5. Theorem 6.1.4 is true when $r=4$.

Proof. We prove this with a computer search, the code for which is in Appendix 6.A.1. This also follows from independent work by Archer [1], Hliněný (personal correspondence), and Pendavingh (personal correspondence).

To prove Theorem 6.1.4, which we do in Section 6.6, we first show that there are only two ways to extend onto a spanning clique in a golden-mean way. We show that in any golden-mean matroid with a spanning clique there must be an element of the clique that is not on a $U_{2,4}$-restriction. In order to prove this, we need to characterise all the ways that a clique element can be on a $U_{2,4}$-restriction, which we do in Section 6.5. We then prove that if a clique element $e$ in a counterexample to Theorem 6.1.4 is not on a $U_{2,4}$-restriction, then $e$ is on at least three non-clique three-point lines. In Section 6.4, we discover that there are four possible configurations that arise when $e$ is on three non-clique three-point lines. We then show that it is not possible to make these configurations big enough to be counterexamples to Theorem 6.1.4, and hence no counterexamples can exist.

In the next definition, we introduce some useful informal language. 
Definition 6.1.6. Let $G$ be a graph, and let $A$ and $B$ be two subgraphs of $G$. We say that $A$ touches $B$ if there exist vertices $v_{A}$ and $v_{B}$ in $A$ and $B$ respectively such that $v_{A}=v_{B}$ in $G$.

\subsection{Red-Green Lemma}

There are two ways to extend onto a clique in a golden-mean way. For simplicity, these are called "green" (corresponding to identifying a $M\left(K_{4}\right)$-restriction of the spanning clique and extending it to an $O_{7}$ ) and "red" (corresponding to identifying a $M\left(K_{4}\right)$-restriction of the spanning clique and extending it to a $\left.F_{7}^{=}\right)$.

First, we need to show that this claim is actually true.

Lemma 6.2.1. If $M$ is a simple golden-mean matroid with a non-coloop element e such that $M \backslash e$ is isomorphic to $M\left(K_{r+1}\right)$, then $e$ is in a line of cardinality three or four.

Proof. Note that if $e$ is in a line, this line cannot have cardinality greater than four, as the maximum size of a line in a graphic matroid is three.

We prove the lemma by induction on $r$. Now consider a minimal counterexample $M$, so $M$ is an extension by $e$ of the graphic matroid $M(G)$, where $G$ is a complete graph on $r+1$ vertices, and $e$ is not on any long lines in $M$. Let $x$ be an arbitrary edge in $G$. Note that in $M / x$, the element $e$ is not in a parallel pair. Let $P$ contain all but one element from each parallel class in $M / x$. So $M / x \backslash P$ is an extension of $M\left(K_{r}\right)$ by $e$. Hence by induction, $e$ is on a line with some elements $y$ and $z$ in $M / x \backslash P$. Therefore $e \in \operatorname{cl}_{M}(\{x, y, z\})$.

Let $V$ be the set of vertices of $G$ incident with at least one of $x, y$, or $z$; and let $G^{\prime}$ be the complete subgraph of $G$ induced by $V$. If we consider $M^{\prime}=M \mid\left(E\left(G^{\prime}\right) \cup\{e\}\right)$, this is a counterexample, as $e$ is a non-coloop element with $M^{\prime} \backslash e$ being the cycle matroid of a complete graph, and $e$ is not in any long lines in $M^{\prime}$. Hence, by minimality, $G^{\prime}=G$, and therefore $r+1$ is at most 6 .

We now have a finite case check, which can be done on a computer. When $r=2$, the proof is trivial. So we only need to check values of $r$ in $\{3,4,5\}$, which is done in Appendix 6.A.2. 
Lemma 6.2.2. The line obtained in Lemma 6.2.1 is unique.

Proof. Take a minimal counterexample $M$. So, in $M$, there exist distinct long lines $\ell_{1}$ and $\ell_{2}$, such that $\ell_{1} \cap \ell_{2}=\{e\}$, and $\left|\ell_{1}\right| \geq\left|\ell_{2}\right|$.

If $\left|\ell_{1}\right|=4$, then we have a 4 -point line (with three clique elements) and a line with at least two clique elements $(f$ and $g$ ) meeting at $e$. Note that $M / f$ contains a four-point line consisting of $\ell_{1}$, with $\{e, g\}$ being a parallel pair. Deleting $e$ from this gives us a graphic matroid with a $U_{2,4}$-restriction, a contradiction [10, Theorem 6.6.7]. Therefore $\left|\ell_{1}\right|=\left|\ell_{2}\right|=3$.

Say that $\ell_{1}=\left\{e, x_{1}, y_{1}\right\}$ and $\ell_{2}=\left\{e, x_{2}, y_{2}\right\}$. This means that $\left\{x_{1}, x_{2}, y_{1}, y_{2}\right\}$ is a four-element cycle in our underlying graph. For $i \in\{1,2\}$, note that $x_{i}$ and $y_{i}$ are not in a triangle, as then $\ell_{i}$ would be a 4 -point line. So there is a $K_{4}$-subgraph of our underlying graph with two edge-disjoint matchings, such that $e$ is on a line with each of them. This makes a Fano or nonFano restriction of $M$, which is a contradiction as neither the Fano nor the non-Fano is golden-mean [10, Page 643].

Because of Lemmas 6.2.1 and 6.2.2, the following definition naturally arises.

Definition 6.2.3. If $M$ is a simple golden-mean matroid with a spanning clique $N$ and an element $e$ such that $M \backslash e$ contains $N$ as a restriction, then $e$ is either in a three-point line or a four-point line $\ell$, such that $\ell \backslash\{e\} \subseteq E(N)$. If $\ell$ is a three-point line we call $e$ a red element, and if $\ell$ is a four-point line we call $e$ a green element.

We would like to be able to describe the green and red elements of $M$ in an useful way - this leads us to the notion of an augmented clique.

Definition 6.2.4. An augmented clique $(G, \mathcal{A})$ is a complete graph $G$ with a multiset $\mathcal{A}$ of sets of edges of $G$, where each member of $\mathcal{A}$ either induces a $K_{3}$ in $G$ or is a two-edge matching of $G$. In the former case we call the member of $\mathcal{A}$ a green triangle, and in the latter case we call the member of $\mathcal{A}$ a red matching.

Definition 6.2.5. Let $C=(G, \mathcal{A})$ be an augmented clique. A restriction of $C$ is an augmented clique $C^{\prime}=\left(G^{\prime}, \mathcal{A}^{\prime}\right)$ such that

(i) $G^{\prime}$ is a complete subgraph of $G$, 
(ii) $\mathcal{A}^{\prime} \subseteq \mathcal{A}$, and

(iii) all members of $\mathcal{A}^{\prime}$ consist of edges of $G^{\prime}$.

In a 3-connected golden-mean matroid $M$ with a spanning clique $N$, the elements of $E(M) \backslash E(N)$ can be partitioned into a green block and a red block. Each element in the green block is in a line with the edges of a green triangle, and each element in the red block is in a line with the edges of a red matching. Thus every golden-mean matroid with a spanning clique gives rise to an augmented clique.

Definition 6.2.6. Every simple golden-mean matroid with a spanning clique corresponds to at least one augmented clique. Any augmented clique that arises in such a manner is a golden-mean augmented clique.

We denote augmented cliques as coloured graphs. These graphs are simple, though we draw them as non-simple graphs to ease comprehension. In other words, if an edge $e$ is contained in a collection of coloured elements, we draw $e$ as a parallel class, with one edge corresponding to each coloured element. For an example, see Figure 6.7.1. We also use the language of non-simple graphs at times.

Note that, as a matroid need not have a unique spanning clique, a given matroid may not give rise to a unique augmented clique.

Not every augmented clique corresponds to a golden-mean matroid. We call such configurations forbidden.

Definition 6.2.7. Let $C$ be an augmented clique. We say that $C$ is forbid$\boldsymbol{d e n}$ if there is no simple golden-mean matroid with a spanning clique, such that the corresponding augmented clique is equivalent to $C$.

\subsection{Forbidden Configurations}

If we have a collection of green triangles and red matchings that is not goldenmean, then any augmented clique containing such a configuration as a subgraph will also not be golden-mean. As such, it is useful to find forbidden configurations of green triangles and red matchings. 
The next lemma, whose proof is trivial, states that restrictions of augmented cliques work as one would expect.

Lemma 6.3.1. Assume $M$ is a golden-mean matroid with spanning clique $M(K)$, and let $(K, \mathcal{A})$ be the corresponding augmented clique. Let $K^{\prime}$ be a complete subgraph of $K$. Let $X$ be the closure of $E\left(K^{\prime}\right)$ in $M$. Then $M \mid X$ is a golden-mean matroid with a spanning clique, and the corresponding augmented clique is equal to the restriction of $(K, \mathcal{A})$ to $K^{\prime}$.

We also need to contract elements of golden-mean augmented cliques.

Lemma 6.3.2. Let $(G, \mathcal{A})$ be a golden-mean augmented clique with corresponding golden-mean matroid $M$, and let $e=u v$ be an edge of $G$. Let $w$ be the vertex produced by identifying $u$ and $v$ in $G / e$. The golden-mean matroid $\operatorname{si}(M / e)$ corresponds to $\left(G^{\prime}, \mathcal{A}^{\prime}\right)$, where $G^{\prime}=\operatorname{si}(G / e)$, and $\mathcal{A}^{\prime}$ consists of the following green triangles and red matchings, up to swapping the labels of $u$ and $v$.

(i) If $X$ is a member of $\mathcal{A}$ that does not touch $u$, then $X$ is a member of $\mathcal{A}^{\prime}$.

(ii) If $T=u x y$ is a member of $\mathcal{A}$, and $T^{\prime}=$ vxy is not a member of $\mathcal{A}$, then the green triangle $T=w x y$ is a member of $\mathcal{A}^{\prime}$.

(iii) If $T=u x y$ is a member of $\mathcal{A}$, and $T^{\prime}=v x y$ is also a member of $\mathcal{A}$, and the green elements corresponding to $T$ and $T^{\prime}$ (for any such $T^{\prime}$ ) in $M / e$ are not parallel, then the green triangle $T=w x y$ is a member of $\mathcal{A}^{\prime}$.

(iv) If $R=(u x, v y)$ is a red matching from $\mathcal{A}$, then the green triangle $T=$ wxy is a member of $\mathcal{A}^{\prime}$, unless this would induce a parallel pair in $M / e$.

Proof. Statements (i), (ii), and (iii) are straightforward. To confirm (iv), consider the $K_{4}$ spanned by $R$. We have shown this in Figure 6.1. The element of $R$ is labelled $r$, and it is on the line spanned by $u x$ and $v y$. The element $e=u v$ is an element inside the $K_{4}$-restriction spanned by $R$. Now, when we contract $u v$ and simplify, we see a matroid isomorphic to $U_{2,4}$, with groundset $\{r, v y, w x, a\}$, where $a$ is an element from the $K_{4}$ spanned by $R$. The result now follows.

All the verifications will be done by Sage [19], by attempting to put all the new elements on top of a complete graph. To optimise this effort, we only 


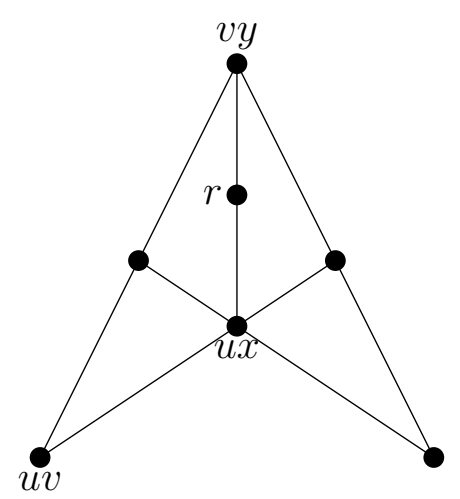

Figure 6.1: $F_{7}^{=}$for Lemma 6.3.2

check one possible location for the configuration. This is acceptable because of the myriad symmetries in complete graphs.

Note that throughout this chapter, when we say that there are $x$ ways to place an element into a clique, we mean $x$ ways, up to whatever symmetries are natural. We make frequent and unstated use of this fact throughout this chapter.

\subsubsection{Complete Graphs}

In order to select a location for the configurations to go, we need to have labelled versions of all the complete graphs we are going to build from. The edge labels for $K_{3}$ are not given as there is only one way to label $K_{3}$. The edge labels for $K_{4}$ are shown in Figure 6.2, the edge labels for $K_{5}$ are shown in Figure 6.3, the edge labels for $K_{6}$ are shown in Figure 6.4, the edge labels for $K_{7}$ are shown in Figure 6.5, and the edge labels for $K_{8}$ are shown in Figure 6.6. These labels come from the standard construction in Sage of the relevant matroid.

\subsubsection{Forbidden Configurations}

First up, we have TR, MR, and PN, shown in Figure 6.7. Next is QI, OM, TL, TB, VQ, and RA, shown in Figure 6.8. Then we have BL, ML, TT, EU, and DJ, shown in Figure 6.9. Next is XS, TM, BC, MW, and DM, shown in Figure 6.10. Following is XG, AE, AF, PL, SW, and DI, shown in 


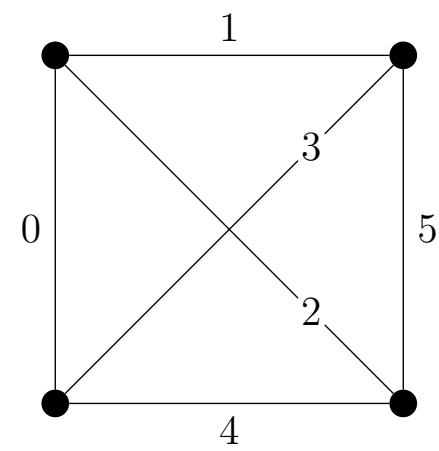

Figure 6.2: Edge Labels for $K_{4}$

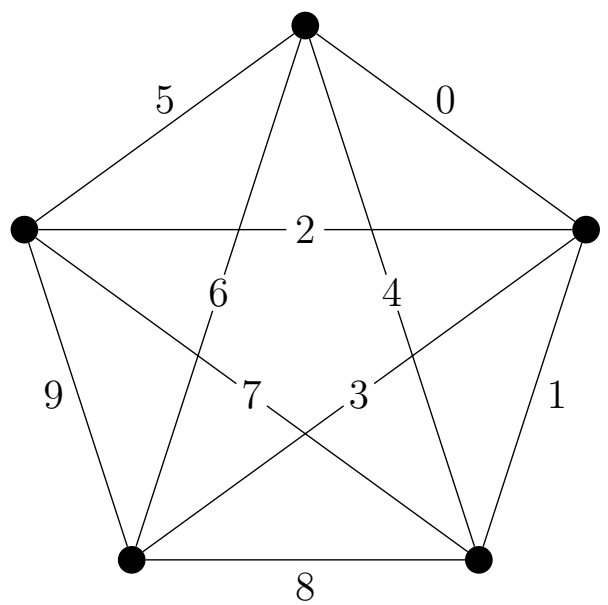

Figure 6.3: Edge Labels for $K_{5}$

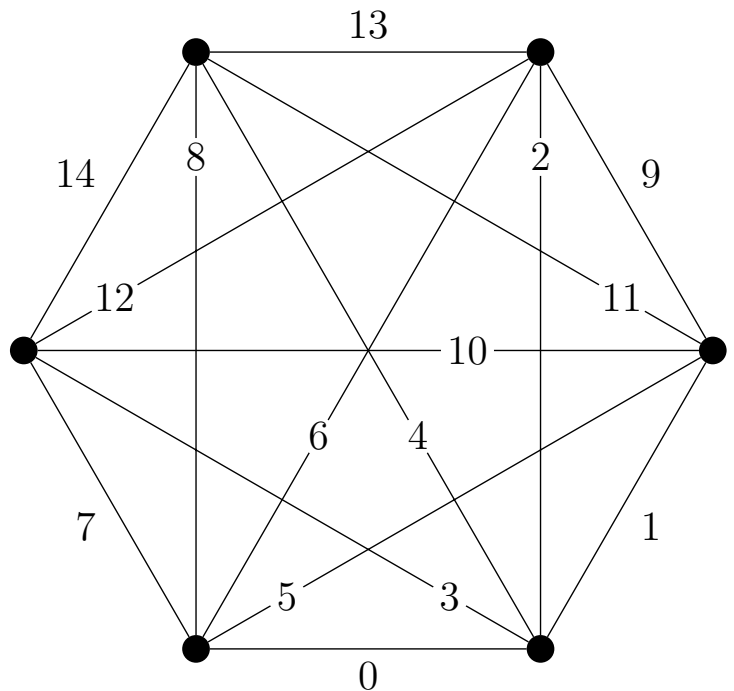

Figure 6.4: Edge Labels for $K_{6}$ 


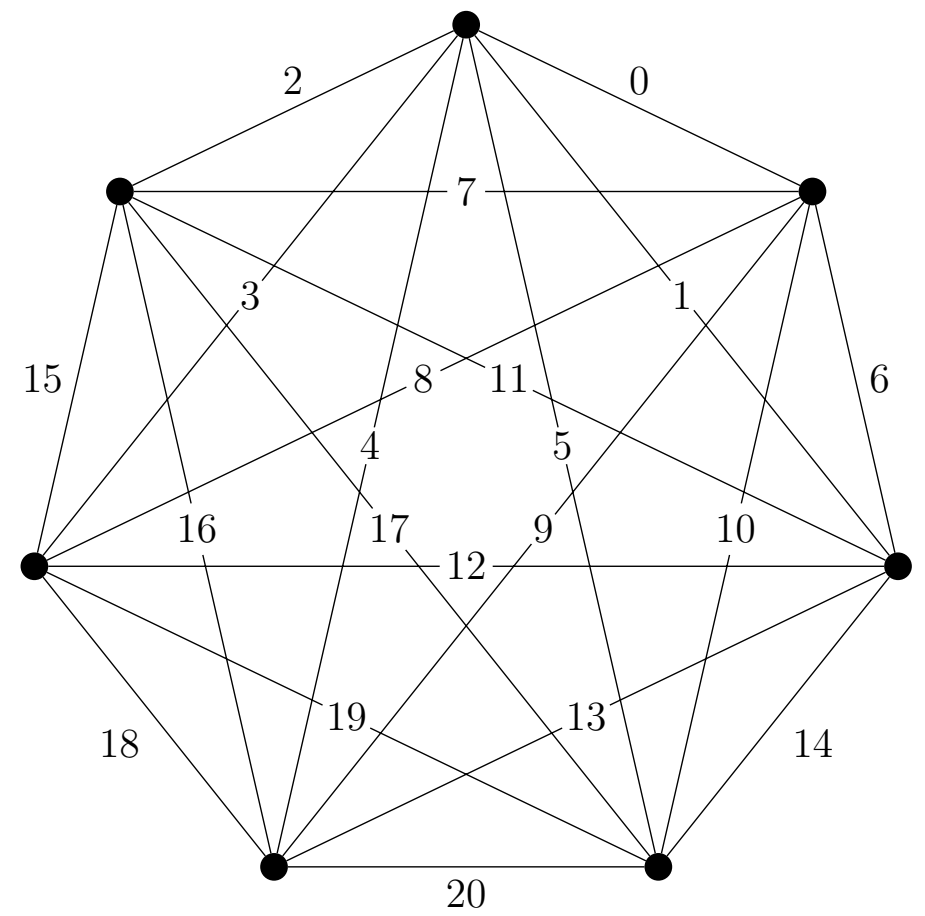

Figure 6.5: Edge Labels for $K_{7}$

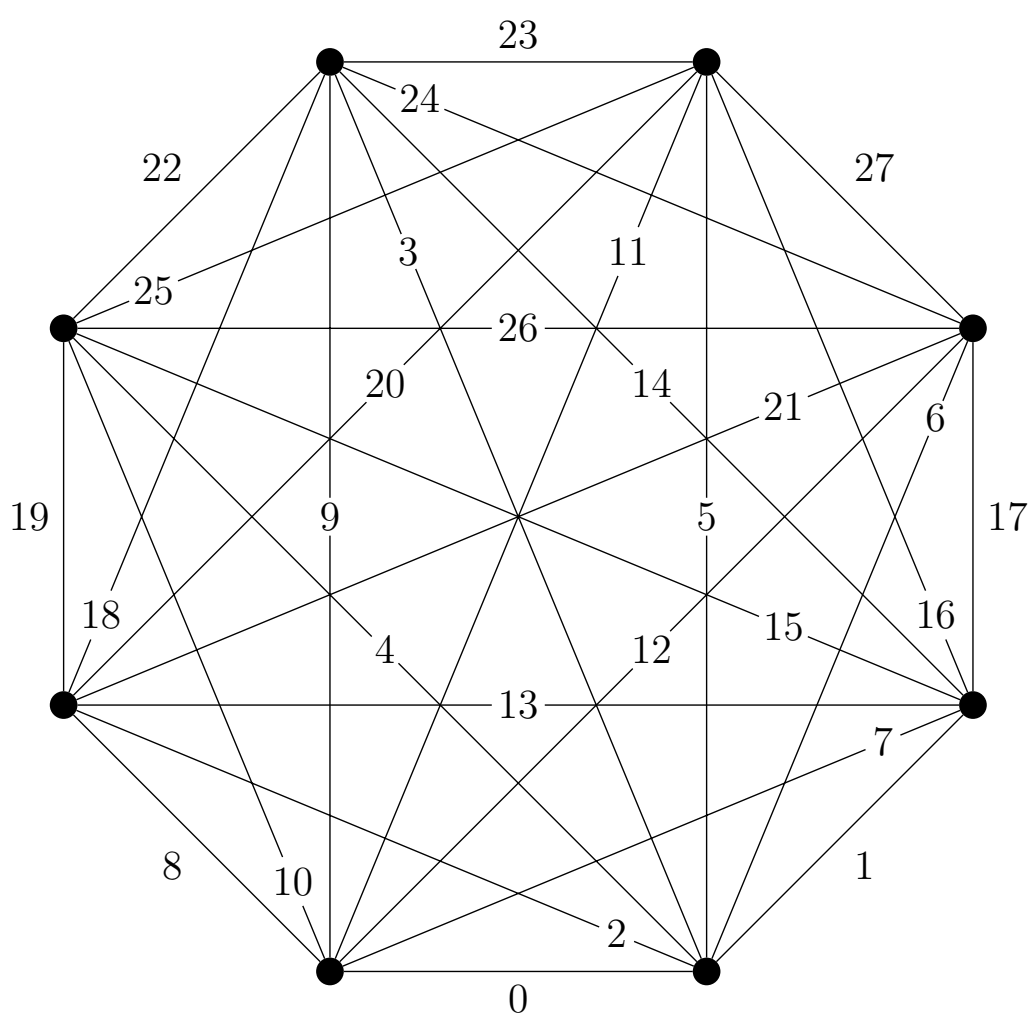

Figure 6.6: Edge Labels for $K_{8}$ 
Figure 6.11. Then we have TP, KF, PM, CC, and CF, shown in Figure 6.12. Finally, we have HN, FS, WB, HM, SR, and BP, shown in Figure 6.13.

We need to verify that these configurations are indeed forbidden.

Lemma 6.3.3. All of the configurations given in Figures 6.7-6.13 are forbidden.

Proof. We run each configuration through the function is_forbidden(), shown in Appendix 6.A.3. The calls to the function are given here. The numbers in the list come from the appropriate labelling of $K_{n}$. For example, to get the numbers for TR, we look at Figure 6.7.1, and note that TR is on five vertices, with one green triangle and one red matching. We now look at the labels for $K_{5}$, Figure 6.3, and note that the triangle in TR corresponds to $\{0,2,5\}$, and the matching to $\{2,8\}$.

TR is_forbidden (5, [ [0, 2, 5], [2, 8] ])

MR is forbidden (6, $[[1,2,9],[7,13]])$

PN is_forbidden (7, [ [0, 2, 7], [14, 18] ])

QI is_forbidden(3, [ [0, 1, 2], [0, 1, 2] , [0, 1, 2] ])

$\mathrm{OM}$ is_forbidden $(5,[[0,2,5],[1,3,8],[7,8,9]])$

TL is_forbidden $(6,[[1,2,9],[7,8,14]])$

TB is_forbidden $(6,[[0,3,7],[1,2,9],[12,13,14]])$

VQ is_forbidden $(6,[[0,4,8],[1,4,11],[7,8,14],[9,11$, $13],[12,13,14]])$

RA is_forbidden (7, $[[0,1,6],[2,3,15],[4,5,20]])$

$\mathrm{BL}$ is_forbidden $(6,[[1,13]$, [4, 7] ])

ML is_forbidden(6, [ [1, 7], [1, 13] ])

TT is forbidden $(6,[[0,9],[7,13]])$

EU is_forbidden (7, [ [0, 14], [2, 18] ])

DJ is_forbidden(8, [ [1, 22], [8, 27] ])

$\mathrm{XS}$ is_forbidden $(4,[[0,5],[1,4],[2,3]])$

TM is_forbidden $(4,[[1,4],[1,4],[1,4]])$

$\mathrm{BC}$ is_forbidden $(5,[[1,5],[1,9],[5,8]])$

MW is forbidden (5, [ [0, 8], $[1,9],[1,9]])$

$\mathrm{DM}$ is_forbidden $(5,[[0,9],[1,9],[5,8]])$

$\mathrm{XG}$ is_forbidden(5, [ [1,9], [3, 7] , [5, 8] ])

$\operatorname{AE}$ is_forbidden $(5,[[0,7],[0,8],[0,9]])$ 

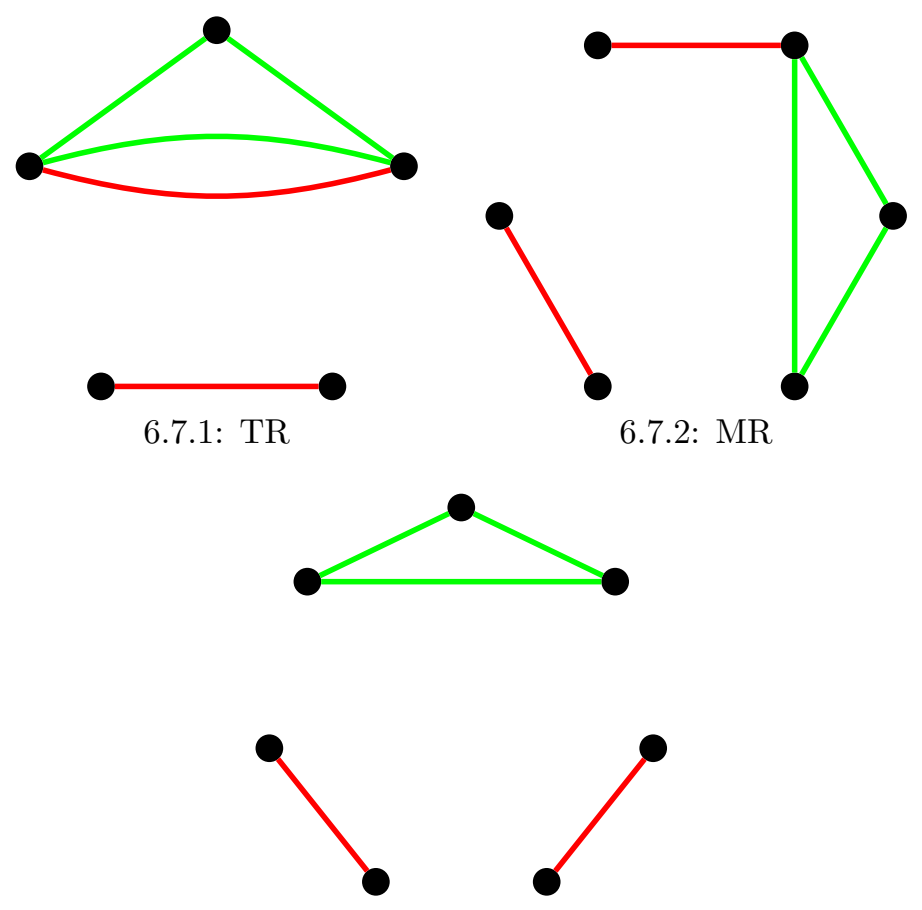

6.7.3: $\mathrm{PN}$

Figure 6.7: Forbidden configurations with one green triangle and one red matching

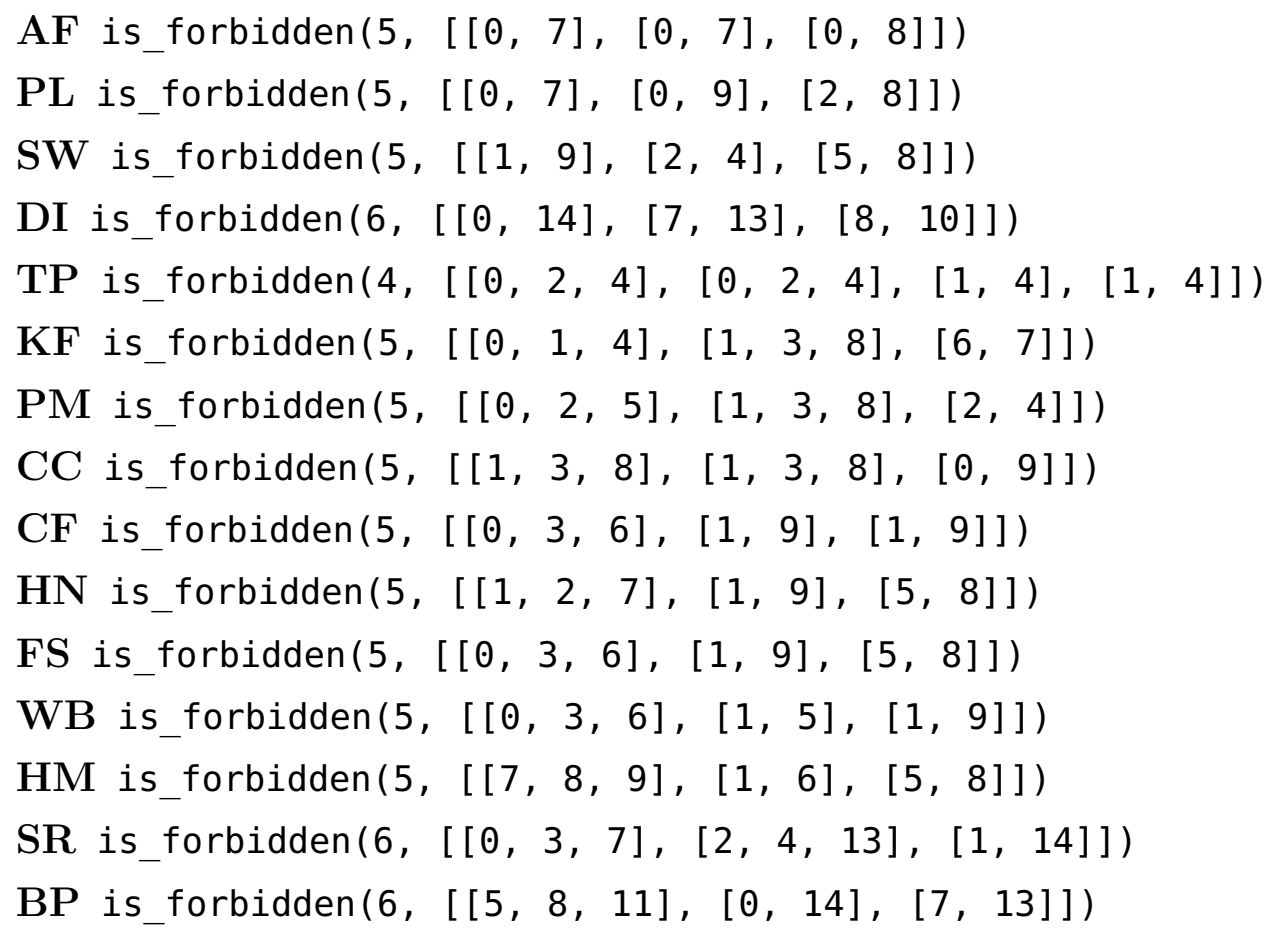

$\operatorname{AF}$ is_forbidden $(5,[[0,7],[0,7],[0,8]])$

PL is_forbidden (5, [ [0, 7] , [0, 9] , $[2,8]])$

SW is forbidden (5, [ [1, 9], $[2,4],[5,8]])$

DI is_forbidden $(6,[[0,14],[7,13],[8,10]])$

TP is forbidden $(4,[[0,2,4],[0,2,4],[1,4],[1,4]])$

$\mathrm{KF}$ is_forbidden (5, $[[0,1,4],[1,3,8]$, $[6,7]])$

$\mathrm{PM}$ is_forbidden (5, $[[0,2,5],[1,3,8],[2,4]])$

$\mathrm{CC}$ is_forbidden $(5,[[1,3,8],[1,3,8],[0,9]])$

$\mathrm{CF}$ is_forbidden (5, [ [0, 3, 6], [1, 9], [1, 9] ])

$\mathrm{HN}$ is_forbidden $(5,[[1,2,7],[1,9],[5,8]])$

FS is_forbidden $(5,[[0,3,6],[1,9],[5,8]])$

$\mathrm{WB}$ is forbidden $(5,[[0,3,6],[1,5],[1,9]])$

$\operatorname{HM}$ is_forbidden $(5,[[7,8,9],[1,6],[5,8]])$

$\operatorname{SR}$ is_forbidden $(6,[[0,3,7],[2,4,13],[1,14]])$

$\mathrm{BP}$ is_forbidden $(6,[[5,8,11],[0,14],[7,13]])$

Lemma 6.3.4. In a golden-mean augmented clique, every edge of every red 
matching must touch every green triangle.

Proof. We will prove this using the three forbidden configurations in Figure 6.7.

Assume that there is a red matching that has an edge that does not touch a green triangle. If the matching is vertex-disjoint from the triangle, we can find a $K_{7}$-restriction of the clique that is a PN (Figure 6.7.3). So only one edge of the matching must be incident with the triangle. This edge can either be incident with the triangle in one vertex (MR, Figure 6.7.2) or two vertices (TR, Figure 6.7.1).

As all other possibilities are forbidden, the statement is true.

Lemma 6.3.5. In a golden-mean augmented clique, every edge of every red matching must touch every other red matching.

Proof. We will prove this using the forbidden configurations in Figure 6.9.

Assume not. So we have a red matching, and we are placing a second red matching so that there is an edge in the second red matching vertex-disjoint from both edges in the first red matching. This can only be done in one way. Now we try to place the final edge of the second red matching. If we place it parallel with an existing red matching edge, we get a ML (Figure 6.9.2). If we place it so it only that it is incident with an existing red matching edge at a single vertex, we get an EU (Figure 6.9.4). If we place it so that it is vertex-disjoint from the existing red matching, we get a DJ (Figure 6.9.5). So it must be incident with both existing red matching edges. There is only one way to do this, which is BL (Figure 6.9.1).

As all other possibilities have been exhausted, the statement is true.

\subsection{Non-clique Three-point Lines}

In order to prove Theorem 6.1.4, we need to understand how non-clique three-point lines and $U_{2,4}$-restrictions behave in the presence of a spanning clique. We start by analysing the non-clique three-point lines. 


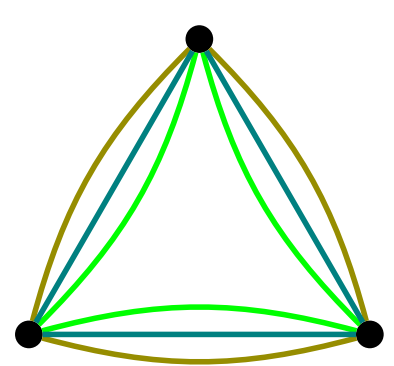

6.8.1: QI

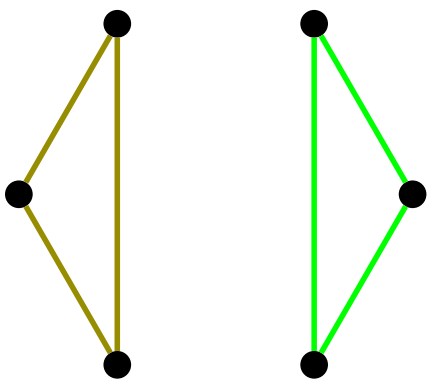

6.8.3: TL

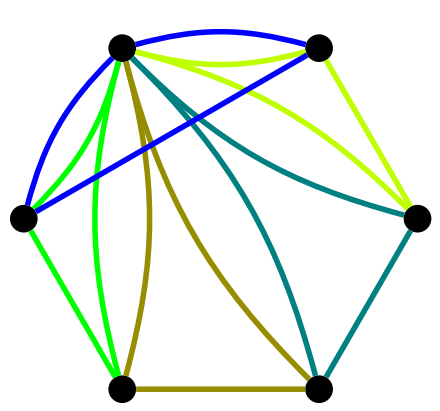

6.8.5: VQ

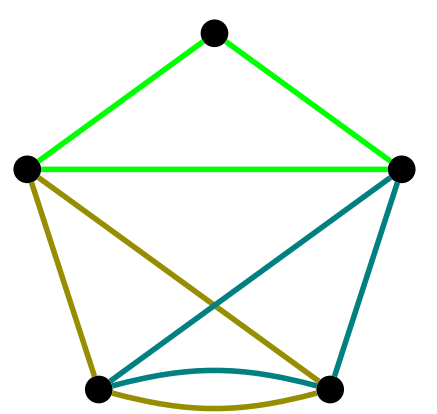

6.8.2: $\mathrm{OM}$

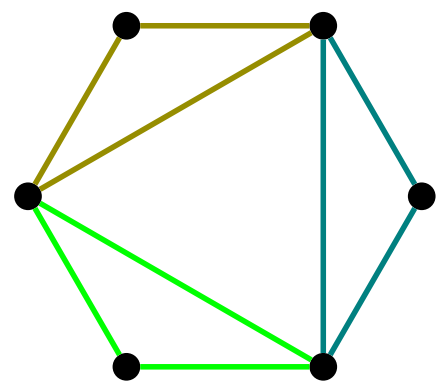

6.8.4: TB

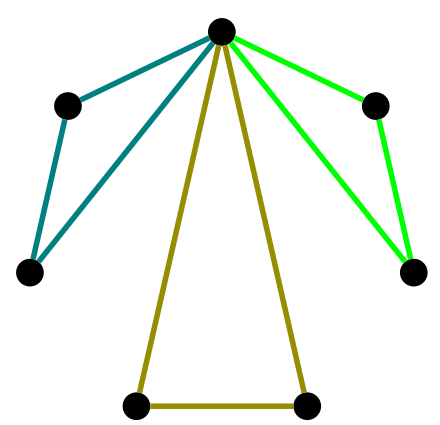

6.8.6: RA

Figure 6.8: Forbidden configurations with only green triangles 


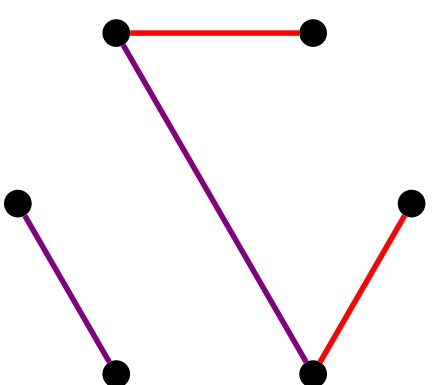

6.9.1: BL

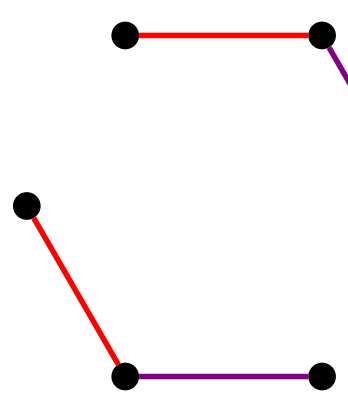

6.9.3: TT
6.9.2: $\mathrm{ML}$
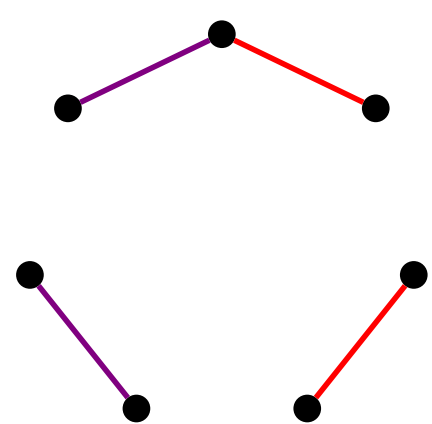

6.9.4: EU
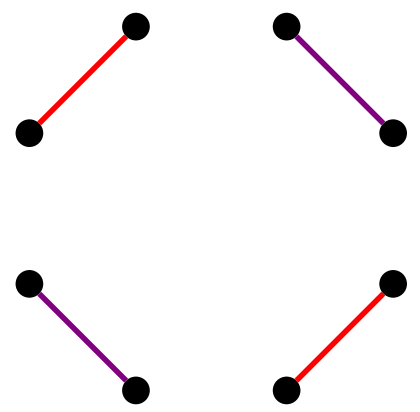

6.9.5: DJ

Figure 6.9: Forbidden configurations with two red matchings 


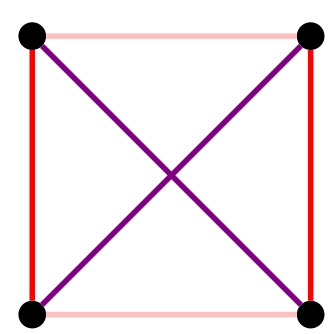

6.10.1: XS

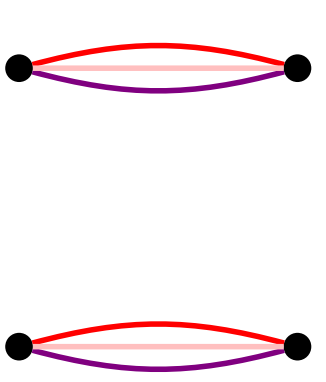

6.10.2: TM

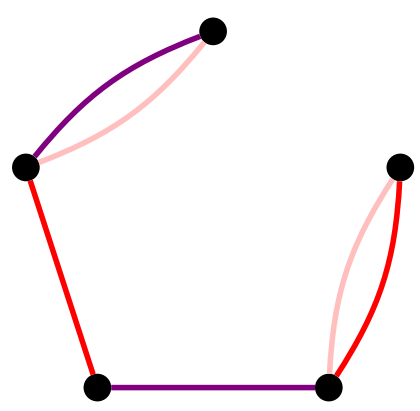

6.10.3: BC

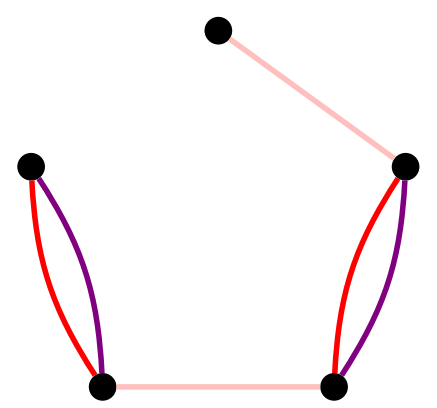

6.10.4: $\mathrm{MW}$

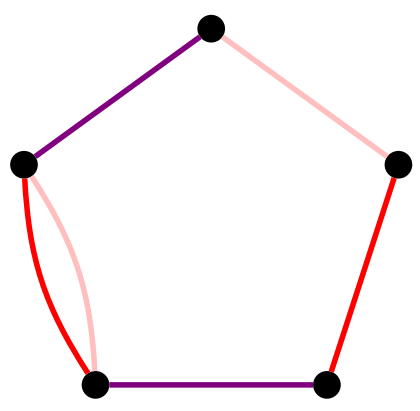

6.10.5: DM

Figure 6.10: Forbidden configurations with three red matchings I

\subsubsection{One Non-clique Three-point Line}

We need to analyse the ways that a clique element can be on a non-clique three-point line.

The non-clique three-point line could have either one or two non-clique elements on it. If it has two non-clique elements, these could be both green, both red, or one red and one green. We consider each in turn, by adding the coloured elements in all possible ways, and then checking to see whether the non-clique elements form a triangle with any clique elements.

\subsubsection{One Non-clique Element}

There are two ways to add in one non-clique element: a red matching or a green triangle. The red matching does create a non-clique three-point line, while the green triangle does not, as lines are flats. 


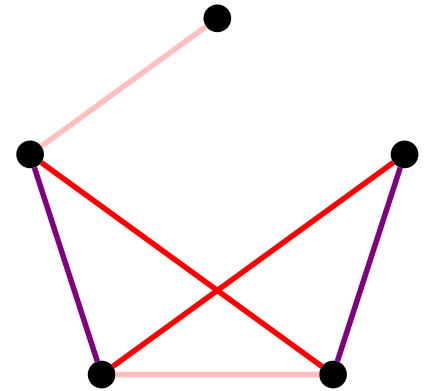

6.11.1: XG

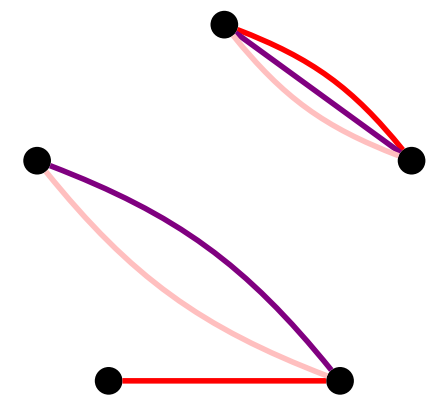

6.11.3: $\mathrm{AF}$

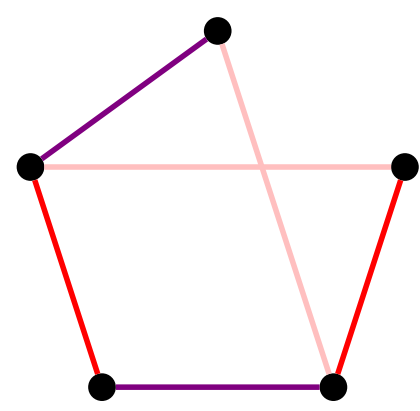

6.11.5: SW

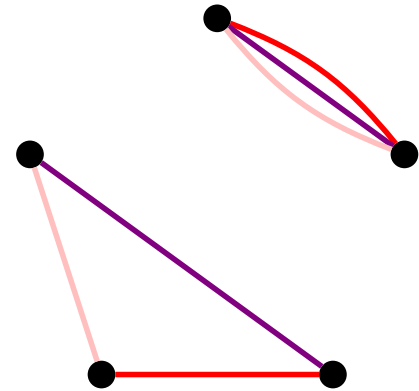

6.11.2: $\mathrm{AE}$

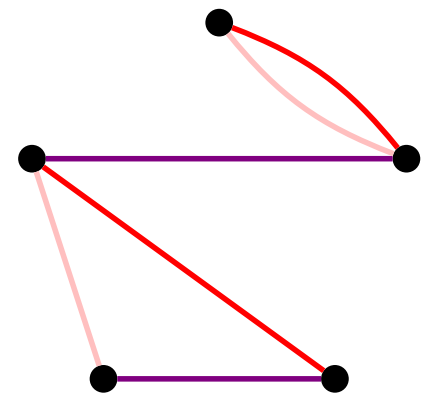

6.11.4: PL

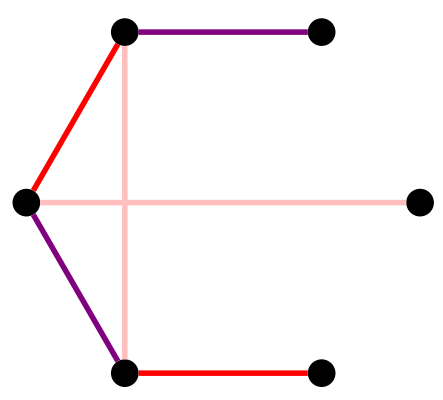

6.11.6: DI

Figure 6.11: Forbidden configurations with three red matchings II 


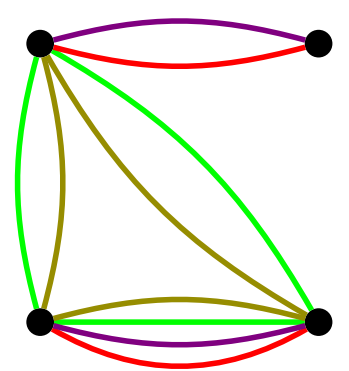

6.12.1: $\mathrm{TP}$

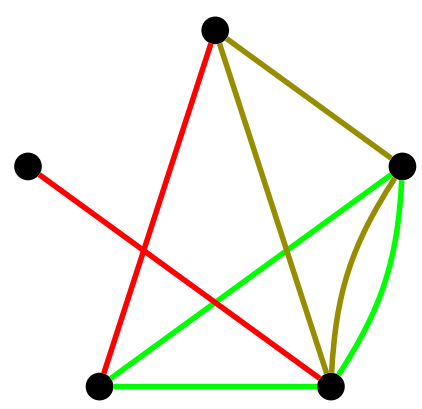

6.12.2: $\mathrm{KF}$

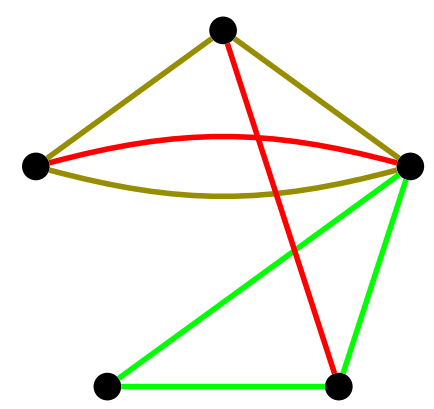

6.12.3: $\mathrm{PM}$

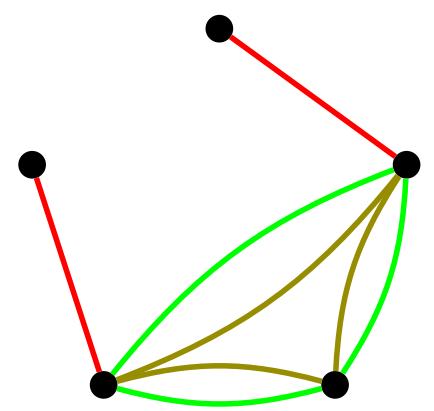

6.12.4: $\mathrm{CC}$

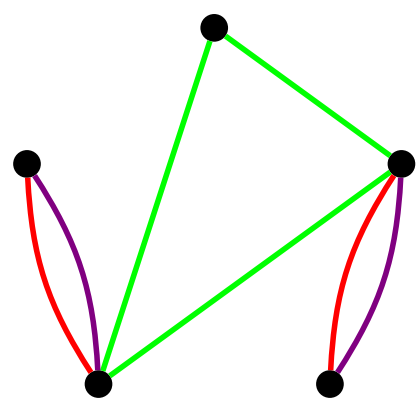

6.12.5: $\mathrm{CF}$

Figure 6.12: Remaining forbidden configurations I

\subsubsection{Two Green Triangles}

Because TL (Figure 6.8.3) is forbidden, the two triangles must not be vertexdisjoint. This leads to three possible configurations: the two triangles meet at a triangle (Figure 6.14.1), an edge (Figure 6.14.2), or a vertex (Figure 6.14.3).

We now go through each configuration in turn and check for the existence of non-clique three-point lines containing both of the extension elements. We also look for $U_{2,4}$-restrictions containing both of the extension elements, as these are required later, in Section 6.5.2. These checks are done by the lines() function from Appendix 6.A.4. We are able to use the smallest spanning clique possible because any line containing the green and red elements must be in the span of said elements. The function calls, and an interpretation of the output are given below.

Triangle lines $(3,[[0,1,2],[0,1,2]])$ outputs that the only line containing both extension elements is the expected five-point line.

Edge lines $(4,[[0,2,4],[1,2,5]])$ outputs that $[a, b, 3]$ is a non-clique three-point line containing both extension elements. This 


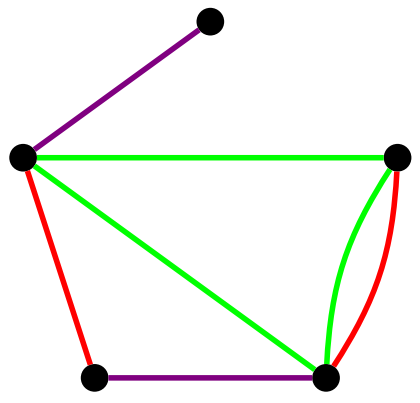

6.13.1: $\mathrm{HN}$

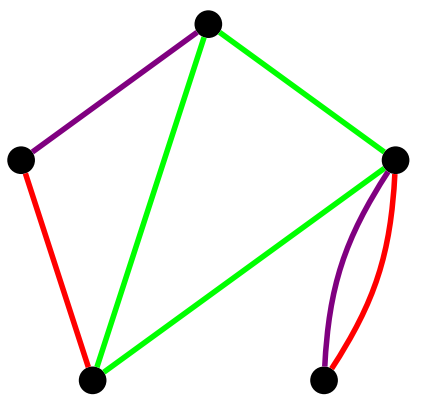

6.13.3: WB

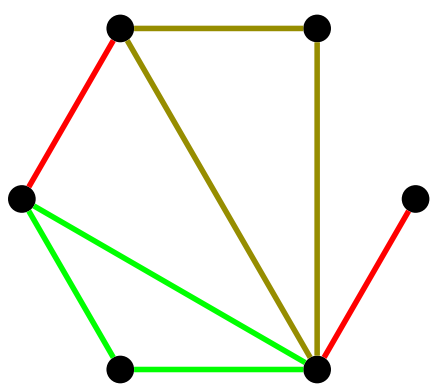

6.13.5: SR

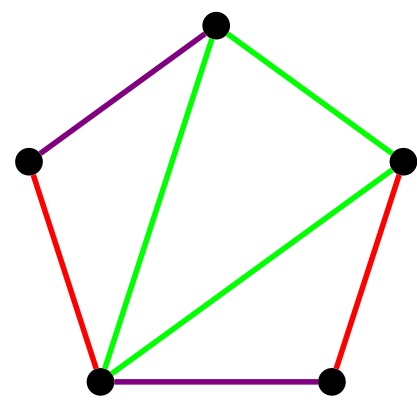

6.13.2: FS

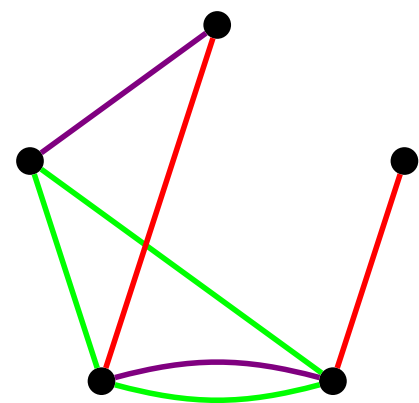

6.13.4: HM

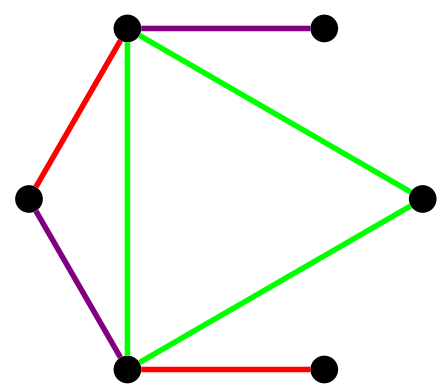

6.13.6: $\mathrm{BP}$

Figure 6.13: Remaining forbidden configurations II

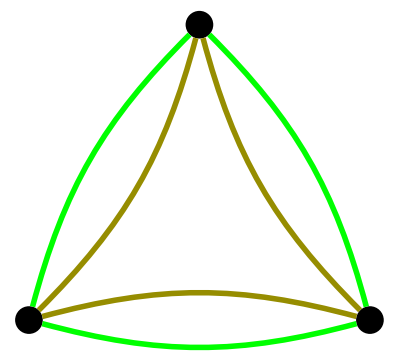

6.14.1: Meet at a triangle

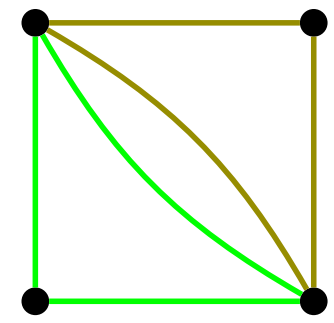

6.14.2: Meet at an edge

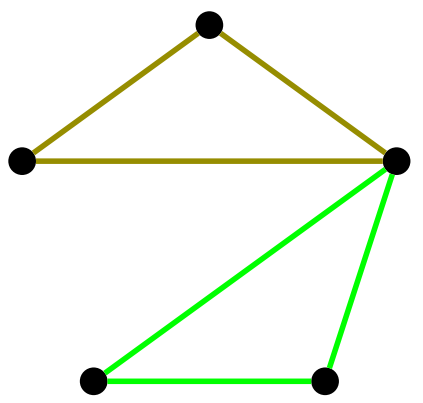

6.14.3: Meet at a vertex

Figure 6.14: Two Green Triangles 
is GG, shown in Figure 6.17.1.

Vertex lines $(5,[[0,2,5],[1,3,8]])$ outputs that there is no line containing both extension elements.

\subsubsection{Two Red Matchings}

We further break the case checking up by number of vertices. First, we analyse all the possible configurations, and then we test each of them in turn for the required properties.

Four vertices We do this systematically. Place the first red matching, there is only one way to do this. Now place the first edge of the second red matching. It can either join the existing red matching edges or it could be parallel to the existing red matching. In both cases there is only one way to put in the final edge. In the first case we get a square of red matchings (Figure 6.15.1), and in the second case we get a double red matching (Figure 6.15.2).

Five vertices As before, there is only one way to place the first red matching, which uses four of the vertices. The first edge of the final red matching must touch the unused vertex, and then there is only one place for it to go. There are now two ways we can add in the final edge: either by joining the two ends of the first red matching (Figure 6.15.3), or by placing an edge in parallel (Figure 6.15.4).

Six vertices The two vertices untouched by the first red matching cannot be joined by an edge in the second red matching, as this would contradict Lemma 6.3.5. This leaves only one way that the second red matching can be added. This is shown in Figure 6.15.5, which is a forbidden configuration (TT, Figure 6.9.3).

Seven or more vertices This is impossible due to Lemma 6.3.5, as the second red matching will need to touch at least three vertices that the first red matching does not touch, meaning at least one edge must be disjoint. 


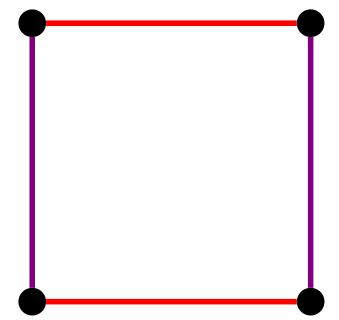

6.15.1: Four vertices square
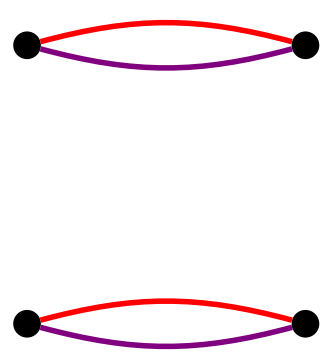

6.15.2: Four vertices double

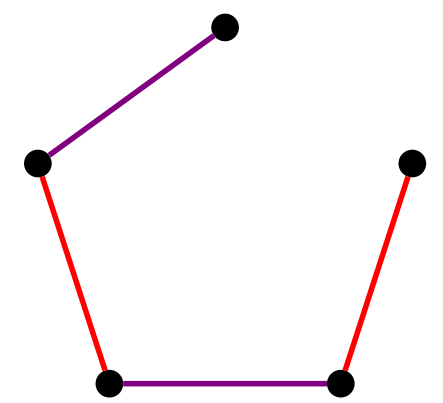

6.15.3: Five vertices - join

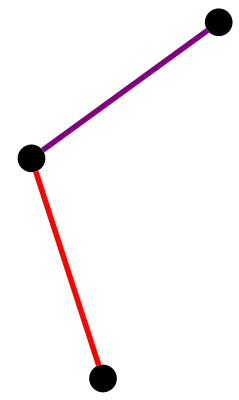

6.15.4: Five vertices - parallel

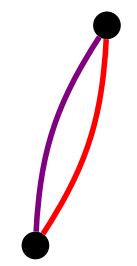

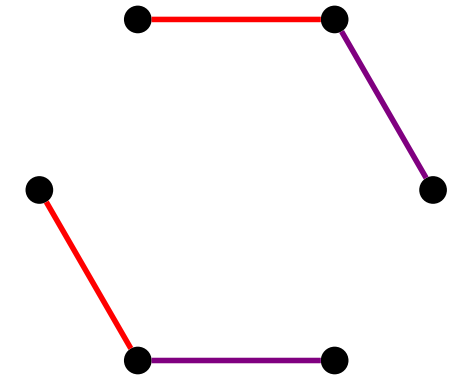

6.15.5: Six vertices

Figure 6.15: Two Red Matchings 


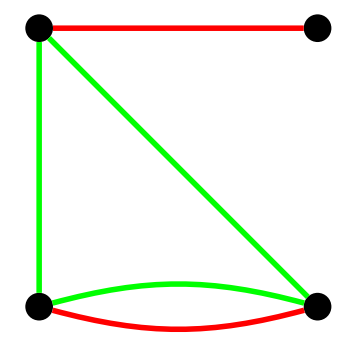

6.16.1: Four vertices

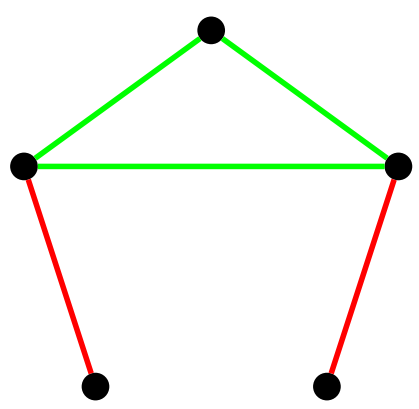

6.16.2: Five vertices

Figure 6.16: One Green Triangle and One Red Matching

We now go through each configuration in turn and check for the existence of non-clique three-point lines containing both of the extension elements. We also look for $U_{2,4}$-restrictions containing both of the extension elements, as these are required later, in Section 6.5.2. These checks are done by the lines() function from Appendix 6.A.4. The function calls, and an interpretation of the output are given below.

Square lines $(4,[[0,5],[1,4]])$ outputs that $[a, b, 3]$ is a nonclique three-point line containing both extension elements. This is RR-square, shown in Figure 6.17.3.

Double lines (4, [ [ 1,4$],[1,4]])$ outputs that the only line containing both extension elements is the expected four-point line.

Join lines $(5,[[1,9],[5,8]])$ outputs that there is no line containing both extension elements.

Parallel lines $(5,[[1,5],[1,9]])$ outputs that $[a, b, 6]$ is a nonclique three-point line containing both extension elements. This is RRdigon, shown in Figure 6.17.4

\subsubsection{One Red Matching and One Green Triangle}

By Lemma 6.3.4, both edges of the matching must touch the triangle. This leads to only two possible configurations, one on four vertices (Figure 6.16.1) and one on five vertices (Figure 6.16.2). 


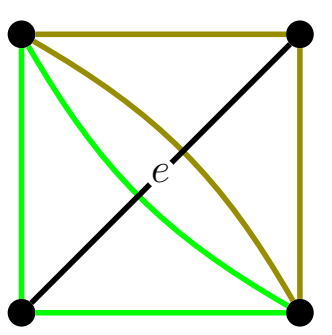

6.17.1: GG

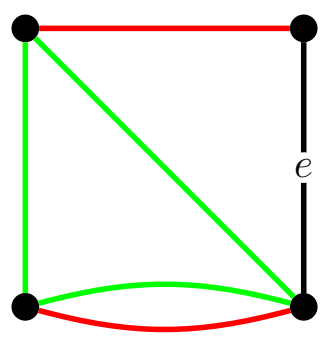

6.17.2: GR

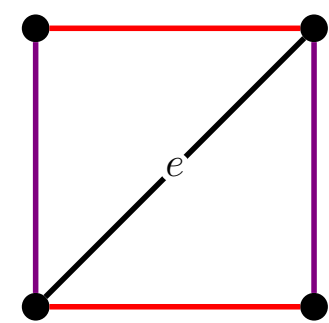

6.17.3: RR-square

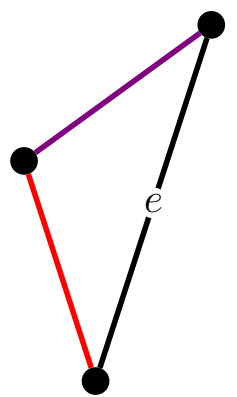

6.17.4: RR-digon

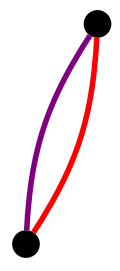

Figure 6.17: One Non-clique Three-point line

We now go through each configuration in turn and check for the existence of non-clique three-point lines containing both of the extension elements. We also look for $U_{2,4}$-restrictions containing both of the extension elements. These checks are done by the lines() function.

Four lines $(4,[[0,2,4],[1,4]])$ outputs that $[a, b, 5]$ is a nonclique three-point line containing both extension elements. This is GR, shown in Figure 6.17.2.

Five lines $(5,[[0,2,5],[1,9]])$ outputs that there is no line containing both extension elements.

\subsubsection{Summary}

In summary, there are five ways that a clique element can be on a non-clique three-point line while not being in a $U_{2,4}$-restriction. Firstly, we could make the clique element part of a red matching. The other four configurations, GG, GR, RR-square, and RR-digon, are shown in Figure 6.17, where $e$ is the distinguished clique element.

As we will be referring to them later, note that there are two components of 
a RR-digon: a parallel component (the two edges in a parallel class), and a path component (the two edges that touch $e$ ).

\subsubsection{Two Non-clique Three-point Lines}

We now want to find all the ways that an element $e$ of the clique can be on two non-clique three-point lines, without being in a $U_{2,4}$-restriction. We have the four configurations in Figure 6.17, as well as the ability to make $e$ part of a red matching, and need to consider the ways to combine these five configurations in order to put $e$ on two non-clique three-point lines. We consider each case in turn.

At no point can we put $e$ into a green triangle, as this would put $e$ onto a four-point line, which we are avoiding.

We use the function all_lines(), described in Appendix 6.A.5 to find all the lines that $e$ is on.

\subsubsection{GG}

Firstly, we start with a GG (Figure 6.17.1), and add in the second line in each of the possible ways.

GG We first consider the case where both lines are GGs.

If we remain on four vertices, there is only one way we can put the second line in, shown in Figure 6.18.1. In this case, all_lines $(4,[[0,2,4],[0$, $2,4],[1,2,5],[1,2,5]], 3)$ outputs that $e$ is on two non-clique three-point lines. We call this configuration $\Gamma$ (Figure 6.24.1).

If we add in one extra vertex, there is also only one way we can put the second line in, shown in Figure 6.18.2. In this case, all_lines (5, [ [0, $1,4],[0,3,6],[1,2,7],[2,3,9]], 8)$ outputs that $e$ is on two non-clique three-point lines. We call this configuration $\Delta$ (Figure 6.24.2).

If we add in two extra vertices, there is also only one way we can put the second line in, shown in Figure 6.18.3. In this case, we can see a TL (Figure 6.8.3), so this configuration is forbidden. As GG has only two vertices disjoint from $e$, this is the largest number of vertices that we can add in. 


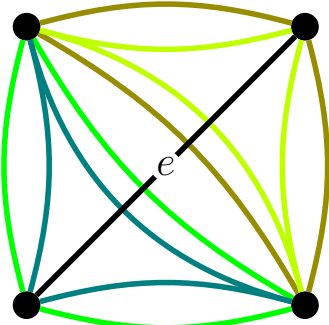

6.18.1: $\mathrm{GG}$ on 4

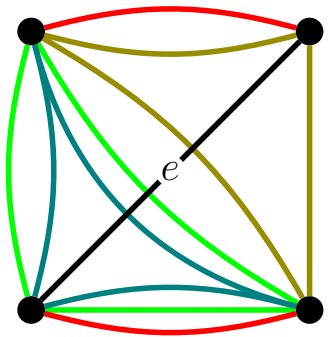

6.18.4: GR on 4

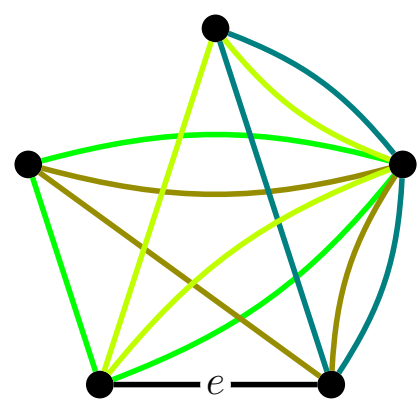

6.18.2: $\mathrm{GG}$ on 5

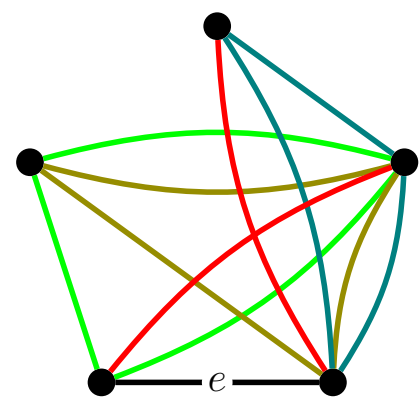

6.18.5: GR on 5 I

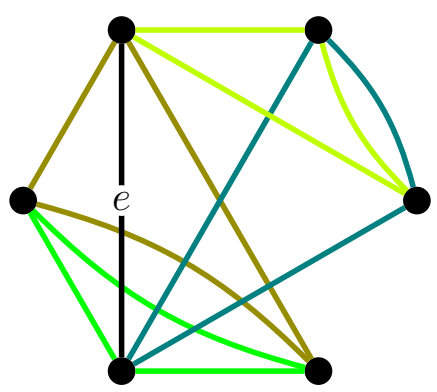

6.18.3: $\mathrm{GG}$ on 6

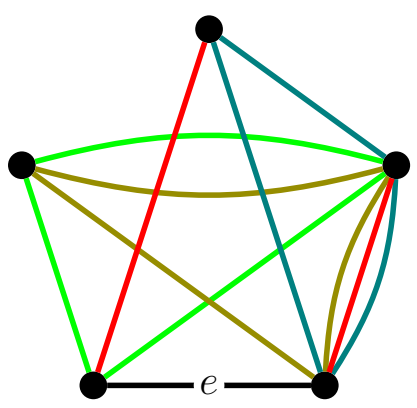

6.18.6: GR on 5 II

Figure 6.18: Starting from a GG I

GR We now start with a GG, and make the second line a GR (Figure 6.17.2).

If we remain on four vertices, there is only one way we can put the second line in, shown in Figure 6.18.4. In this case, all_lines $(4,[[0,2,4],[0,2$, $4],[1,2,5],[1,4]], 3)$ outputs that $e$ is on a four-point line, so we discard this case.

If we add in one extra vertex, then there is only one location for the green triangle in the GR. After this is placed, there are two places we could put the red matching, shown in Figures 6.18.5 and 6.18.6. Both configurations contain a red matching edge disjoint from a triangle, and hence are forbidden by Lemma 6.3.4.

If we add in two extra vertices, there is only one way we can put the second line, shown in Figure 6.19.1. This contains a matching edge disjoint from a triangle, and hence is forbidden by Lemma 6.3.4.

RR-square We now start with a GG, and make the second line a RRsquare (Figure 6.17.3). We also take Lemma 6.3.4 into account, as it reduces 
the number of locations that a matching edge could go.

Regardless of how many vertices we have, the red matching edges must all be incident with both green triangles. Furthermore, each red matching edge must also be incident with $e$. This leaves only one possible location for the RR-square to go, shown in Figure 6.19.2. In this case, al __lines $(4,[[0,2,4],[1,3,5],[0,5],[1,4]], 3)$ outputs that, among other cases, it is possible for $e$ to be on two non-clique three-point lines. We call this configuration $\Theta$ (Figure 6.24.3).

RR-digon We now start with a GG, and make the second line a RR-digon (Figure 6.17.4).

No matter how many vertices we have, the red matching edges must all be incident with both green triangles. As the edges of the path component of the RR-digon must also be incident with $e$, there is only one place that they can go. This leaves one vertex that the parallel class must be incident with, giving the configuration shown in Figure 6.19.3. In this case, all_lines (5, $[[1,3,8],[2,3,9],[0,8],[0,9]], 7)$ outputs that $e$ is on two non-clique three-point lines. We call this configuration $\Lambda$ (Figure 6.24.4).

$e$ in a red matching We now start with GG, and form the second nonclique three-point line by making $e$ part of a red matching.

The first matching edge must go parallel with $e$. As the last matching edge must be incident with both green triangles, there are two possible configurations. The first configuration, on four vertices, in shown in Figure 6.19.4. In this case, all_lines $(4,[[0,2,4],[1,3,5],[2,3]], 3)$ outputs that, among other cases, it is possible for $e$ to be on two non-clique three-point lines. We call this configuration $\Xi$ (Figure 6.24.5). The second configuration, on five vertices, is shown in Figure 6.19.5. This is KF (Figure 6.12.2), and so is forbidden.

\subsubsection{GR}

We now start from a GR (Figure 6.17.2) and add in the second line in each of the possible ways, with the exception of GG, as that case has already been 


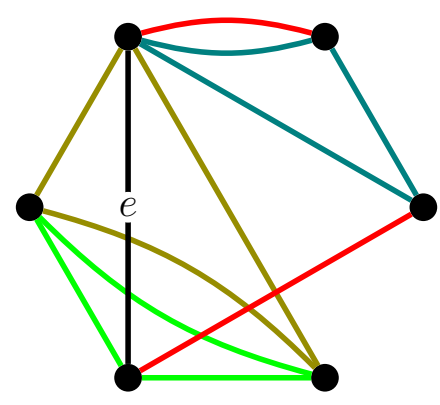

6.19.1: GR on 6

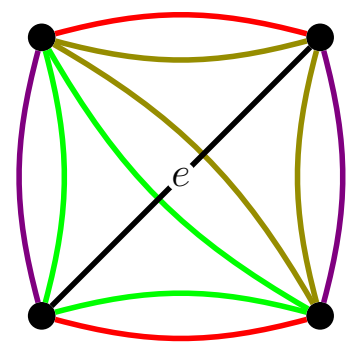

6.19.2: RR-square

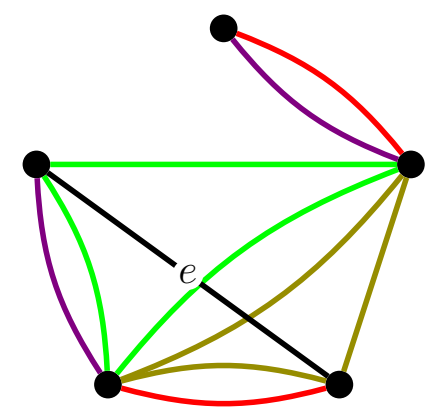

6.19.3: RR-digon

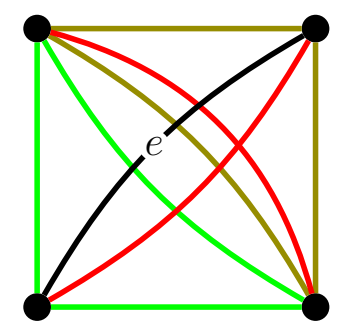

6.19.4: $e$ in a red matching on 4

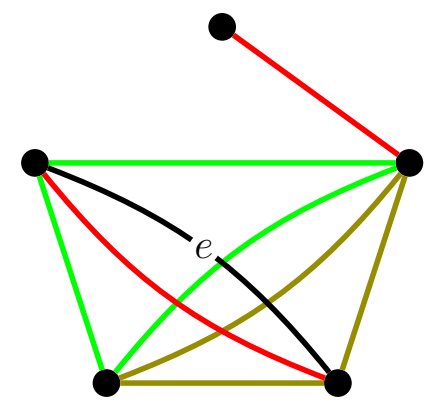

6.19.5: $e$ in a red matching on 5

Figure 6.19: Starting from a GG II

covered.

GR First, we consider the case where both lines are GRs. If this is on four vertices, we can either place the second GR on top of the first GR, as shown in Figure 6.20.1, or flip it over the perpendicular bisector of $e$, as shown in Figure 6.20.2. In the first case, we note that it is TP (Figure 6.12.1), and so is forbidden. In the second case, all_lines $(4,[[0,2,4],[3,4,5]$, $[0,5],[0,5]], 1)$ outputs that $e$ is on two non-clique three-point lines. We call this configuration $\Pi$ (Figure 6.24.6).

We now consider the case where both lines are GRs, and we are on five vertices. The second green triangle can either be incident with the vertex where the first green triangle touches $e$, or it could not. If it is, as each green triangle must touch every matching edge, there is only one location for it. Furthermore, out of the two possible locations of the red matching, only one does not contradict Lemma 6.3.4, and so the only possible configuration is shown in Figure 6.20.3. Now, if the second green triangle is not incident with 
the vertex where $e$ meets the first green triangle, by similar reasoning, there is also only one place for the second GR to go, shown in Figure 6.20.4. In the first case, all_lines $(5,[[0,3,6],[1,3,8],[0,9],[1,9]], 2)$ outputs that $e$ is on no non-clique three-point lines, so this case is discarded. In the second case, this configuration contains an HM (Figure 6.13.4), and so is forbidden.

When we try to place two GRs on six vertices, we quickly arrive at a TT (Figure 6.9.3), and so this is forbidden.

RR-square We now consider the case where the first line is a GR and the second line is a RR-square (Figure 6.17.3). On four vertices, there is only one way to do this, shown in Figure 6.20.5. In this case, all_lines (4, $[0,2,4],[1,4],[1,4],[2,3]], 5)$ outputs that $e$ in on a fourpoint line, so this case is discarded. If we try to do the same pairing on five (or more) vertices, there must be a matching edge incident with a new vertex and the vertex that is incident with $e$ but not incident with the existing green triangle. This matching edge is vertex-disjoint from the existing green triangle, a contradiction to Lemma 6.3.4.

RR-digon The next case is where the first line is a GR and the second line is a RR-digon (Figure 6.17.4). By the same reasoning as in the previous case, the path component of the digon must be in the span of the GR, and hence there are two possible locations for the path. As the parallel component must be incident with the green triangle, once the path is placed, there is only one location remaining for the parallel component. These two configurations are shown in Figures 6.20.6 and 6.20.7. In each configuration, we can find a BC (Figure 6.10.3), and so both configurations are forbidden.

$e$ in a red matching Finally, we start with a GR, and make $e$ part of a red matching. On four vertices, there is only one way to do this, shown in Figure 6.20.8. In this case, all_lines $(4,[[0,2,4],[0,5],[1,4]]$, 5 ) outputs that, among other cases, $e$ is on two non-clique three-point lines. We call this configuration $\Sigma$ (Figure 6.24.7). We now do this on five vertices. The last red matching edge can either be incident with the edge that both the existing green triangle and existing red matching use (Figure 6.20.9), or it 
could not be (Figure 6.20.10). In the first case, this is a HM (Figure 6.13.4), and in the second case this is a HN (Figure 6.13.1). So both cases are forbidden.

\subsubsection{RR-square}

We now start from a RR-square (Figure 6.17.3) and add in the second line in each of the possible ways, with the exception of GG and GR, as those cases have already been covered.

RR-square We start with both lines being a RR-square. There is only one way to do this on each of four (Figure 6.21.1), five (Figure 6.21.2), and six (Figure 6.21.3) vertices. In the four vertex case, all_lines (4, [ [0, 5], $[0,5],[1,4],[1,4]], 3)$ outputs that $e$ is on two non-clique threepoint lines. We call this configuration $\Upsilon$ (Figure 6.24.8). In the five vertex case, all_lines $(5,[[0,8],[1,6],[1,9],[2,8]], 3)$ outputs that $e$ is on two non-clique three-point lines. We call this configuration $\Phi$ (Figure 6.24.9). In the six vertex case, we are able to see a TT (Figure 6.9.3), and so this case is forbidden.

RR-digon The next case is when the first line is a RR-square and the second line is a RR-digon (Figure 6.17.4). If we do this on five vertices, either the parallel or the path component of the RR-digon can be incident with the new vertex. In the first case (Figure 6.21.4), we can find a BC (Figure 6.10.3), and so this is forbidden. In the second case (Figure 6.21.5), we can find a DM (Figure 6.10.5), and so this is forbidden. On six vertices, both the parallel and path components of the RR-digon need to be incident with new vertices, which leaves only one possibility, Figure 6.21.6. In this case, we can see a TT (Figure 6.9.3), and so this case is forbidden.

$e$ in a red matching Finally, we consider the case where the first line is a RR-square and $e$ is part of a red matching. If we are to not contradict Lemma 6.3.5, then we can either do this on four (Figure 6.21.7), or five (Figure 6.21.8) vertices. In the four vertex case, we get XS (Figure 6.10.1), and 


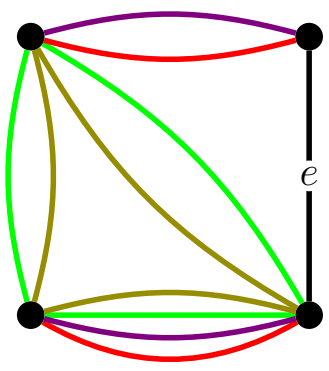

6.20.1: GR on 4 I

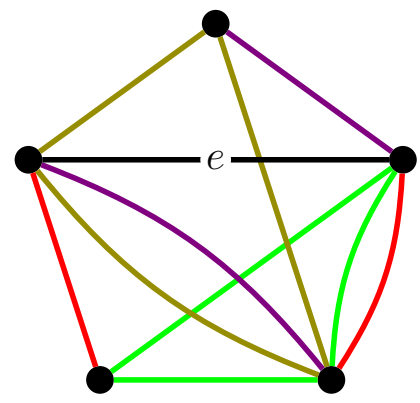

6.20.4: GR on 5 II

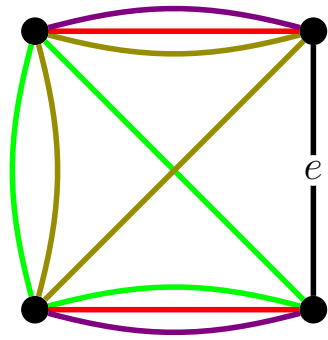

6.20.2: GR on 4 II

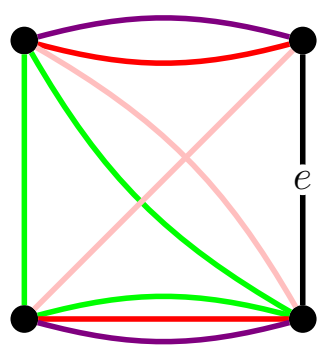

6.20.5: RR-square on 4

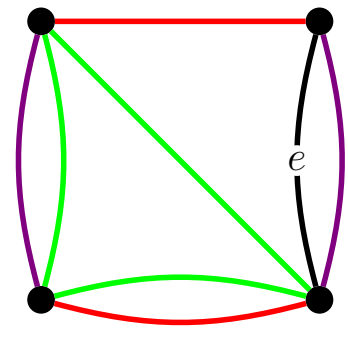

6.20.8: $e$ in a red matching on 4 on 5 II

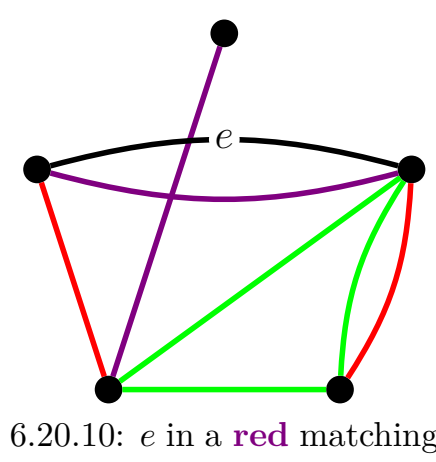

6.20.10: $e$ in a red matching

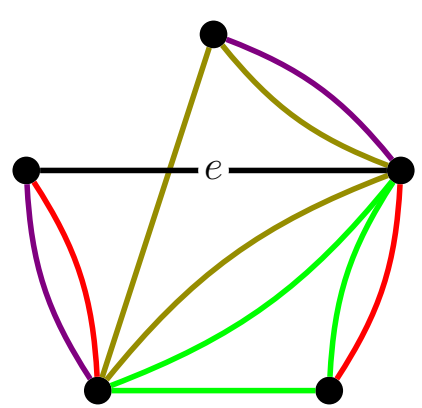

6.20.3: GR on 5 I

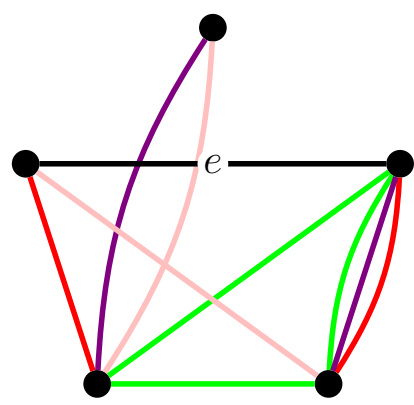

6.20.6: RR-digon I

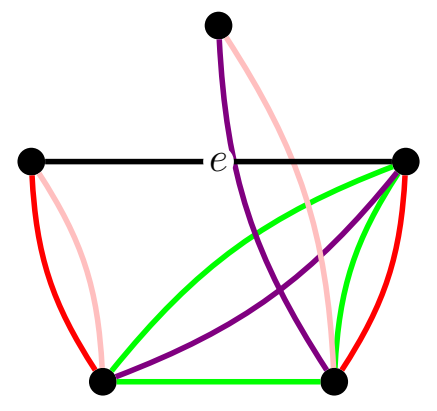

6.20.7: RR-digon II

Figure 6.20: Starting from a GR 
so this case is forbidden. In the five vertex case, we get XG (Figure 6.11.1), and so this case is forbidden.

\subsubsection{RR-digon}

We now start from a RR-digon (Figure 6.17.4) and add in the second line in both of the remaining possible ways, which are as a RR-digon and by having $e$ be in a red matching.

RR-digon First, we consider the case where both lines arise from a RRdigon. We do this case slightly differently from the previous cases.

We start by placing the path component of the second RR-digon. The end vertices of this component must be joined by $e$. This could go parallel to the existing path component, it could touch the existing parallel component, or it could be vertex-disjoint from the first RR-digon. In this last case, it is possible to find a contradiction to Lemma 6.3.5, so we discard it.

Next, we consider the case where the path component of the second RRdigon is parallel to the path component of the first RR-digon. As the parallel component of the second RR-digon cannot be disjoint from the existing RR-digon by Lemma 6.3.5, there are two locations that we could place it. First, we could place it in parallel to the existing parallel component, giving Figure 6.22.1. In this case, we can find an AF (Figure 6.11.3), and so this case is forbidden. Second, we could place it incident with the existing parallel component, giving Figure 6.22.2. In this case, we can find a TT (Figure 6.9.3), and so this case is forbidden.

Finally, we consider the case where the path component of the second RRdigon touches the existing parallel component. There are now three locations for the parallel component of the second RR-digon to go. First, it could touch only the path component of the first RR-digon, giving Figure 6.22.3. In this case, we can find a TT (Figure 6.9.3), and so this case is forbidden. Secondly, the parallel class of the second RR-digon could touch only the parallel class of the first RR-digon, giving Figure 6.22.4. In this case, the edges in the path component of the first RR-digon and the new parallel class give a contradiction to Lemma 6.3.5, and so this case is forbidden. Finally, we could place the parallel component of the second RR-digon so that it touches 


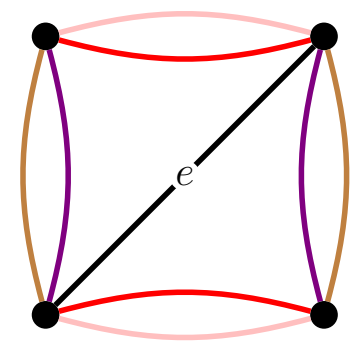

6.21.1: RR-square on 4

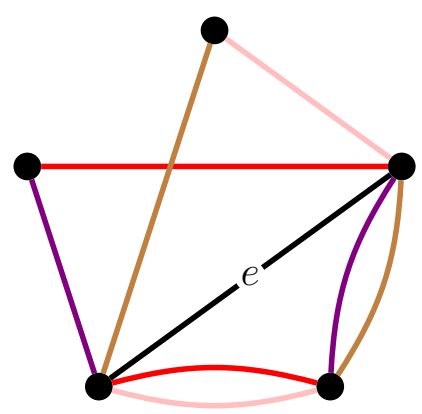

6.21.2: RR-square on 5

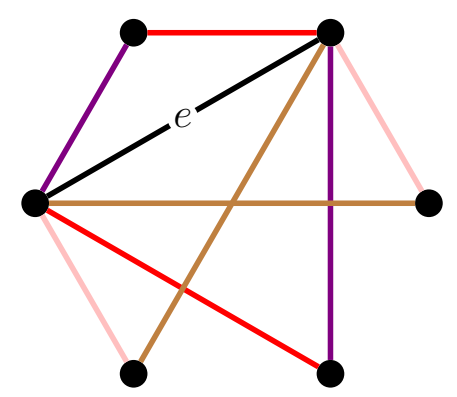

6.21.3: RR-square on 6

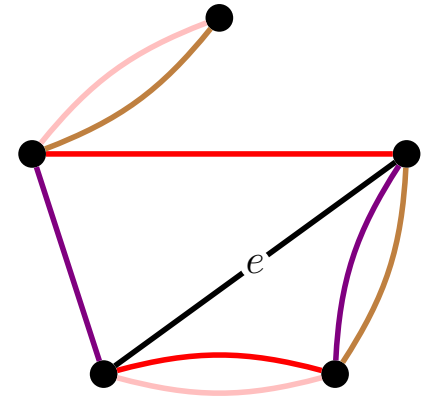

6.21.4: RR-digon on $5 \mathrm{I}$

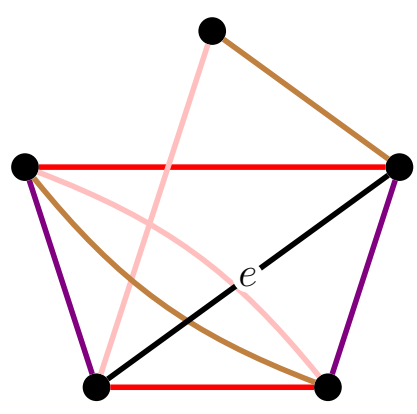

6.21.5: RR-digon on 5 II

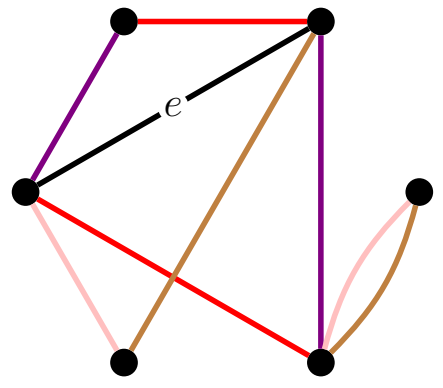

6.21.6: RR-digon on 6

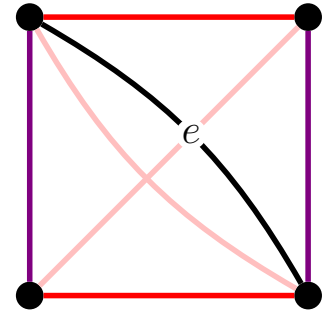

6.21.7: $e$ in a red matching on 4

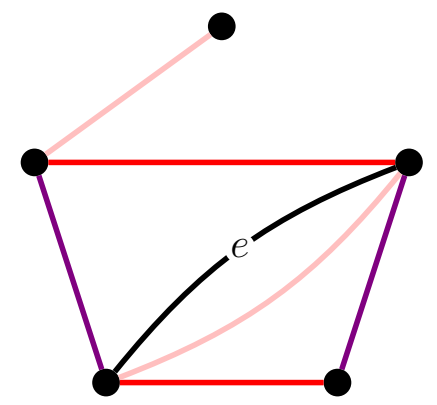

6.21.8: $e$ in a red matching on 5

Figure 6.21: Starting from a RR-square 
both the path and parallel components of the first RR-digon, as shown in Figure 6.22.5. In this case, all_lines (5, [ [ 1, 5], [1, 9], [2, 4], [2, 8] ], 6) outputs that $e$ is on two non-clique three-point lines. We call this configuration $\Psi$ (Figure 6.24.10).

$e$ in a red matching We now consider the case where the first line is a RR-digon and the second line arises from $e$ being part of a red matching. As the second edge of the new red matching cannot avoid the existing parallel component (because of Lemma 6.3.5), there are three places we could place this last edge. Firstly, we could place it so that it is in the existing parallel class, as shown in Figure 6.22.6. This is AE (Figure 6.11.2), and so is forbidden. Secondly, we could place the final edge so that it is incident with the existing parallel class and a vertex outside of the existing RR-digon, as shown in Figure 6.22.7. In this case, we can find a TT (Figure 6.9.3), and so this case is forbidden. Ultimately, we can add the final edge in so that it is incident with both the existing path and parallel components, as shown in Figure 6.22.8. This is PL (Figure 6.11.4), and so is forbidden.

\subsubsection{5 $e$ in a matching}

The final case to consider is when $e$ is in two red matchings. As matching edges cannot be disjoint by Lemma 6.3.5, there are only two ways to have $e$ in two matchings. The first is to identify the two matchings, shown in Figure 6.23.1. In this case, all_lines (4, [ [1, 4], [1, 4] ], 4) outputs that $e$ is on a four-point line, so this case is discarded. The final configuration is when the two matching edges not parallel with $e$ form a path, as shown in Figure 6.23.2. In this case, all_lines (5, [ [1, 5], [1, 9] ], 1) outputs that $e$ is on two non-clique three-point lines. We call this configuration $\Omega$ (Figure 6.24.11).

\subsubsection{Conclusion}

In summary, there are eleven ways that $e$ can be on two non-clique threepoint lines: $\Gamma, \Delta, \Theta, \Lambda, \Xi, \Pi, \Sigma, \Upsilon, \Phi, \Psi$, and $\Omega$. These are shown in Figure 6.24. 


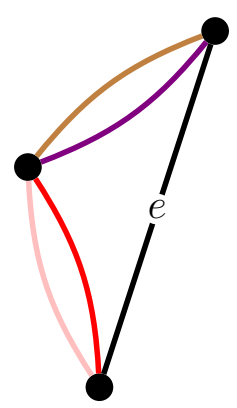

6.22.1: RR-digon I

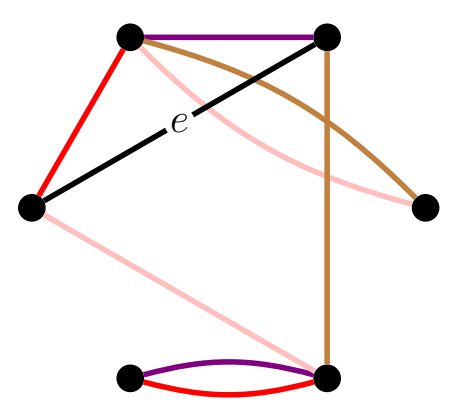

6.22.3: RR-digon III

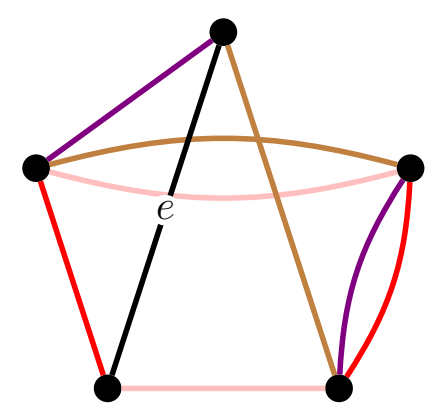

6.22.5: RR-digon V

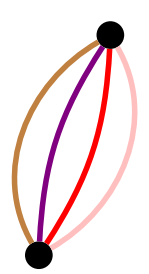

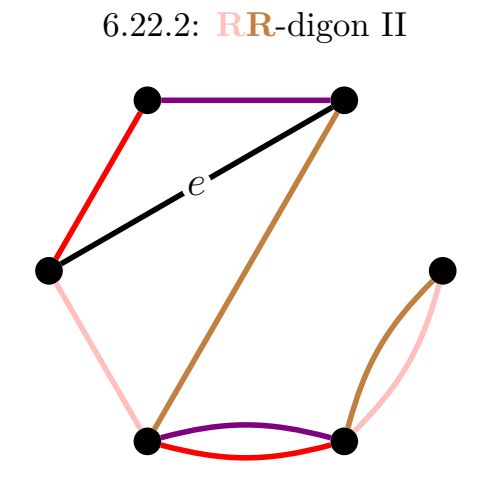

6.22.4: RR-digon IV

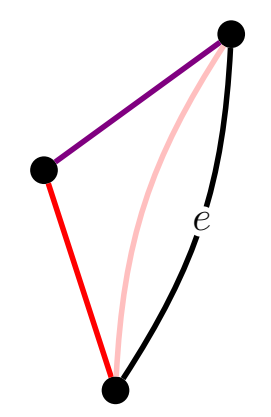

6.22.6: $e$ in a red matching I
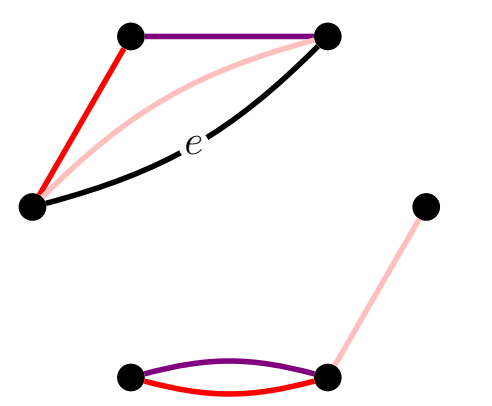

6.22.7: $e$ in a red matching II
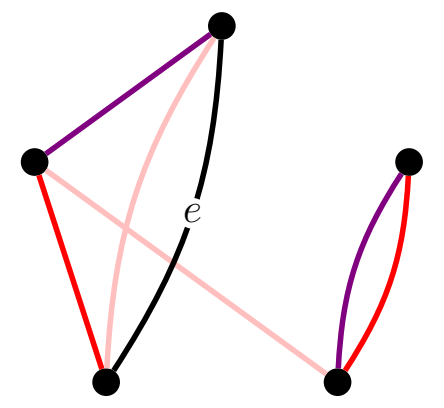

6.22.8: $e$ in a red matching III

Figure 6.22: Starting from a RR-digon 


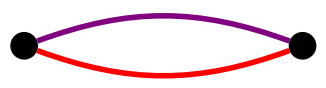

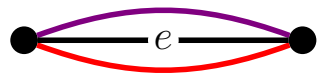

6.23.1: Configuration I

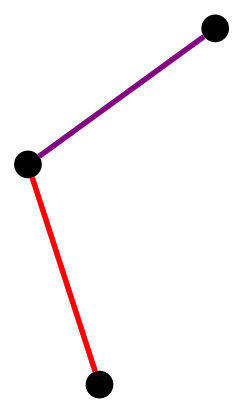

6.23.2: Configuration II

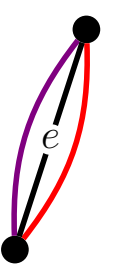

Figure 6.23: Starting from $e$ in a red matching

\begin{tabular}{c||c|c|c|c|c} 
& GG & GR & RR-square & RR-digon & $e$ red \\
\hline \hline GG & $\Gamma, \Delta$ & & $\Theta$ & $\Lambda$ & $\Xi$ \\
\hline GR & & $\Pi$ & & & $\Sigma$ \\
\hline RR-square & $\Theta$ & & $\Upsilon, \Phi$ & & \\
\hline RR-digon & $\Lambda$ & & & $\Psi$ & \\
\hline$e$ red & $\Xi$ & $\Sigma$ & & & $\Omega$
\end{tabular}

Table 6.1: How the eleven ways to get $e$ on two non-clique three-point lines (Figure 6.24) arise from the five ways to have $e$ on one non-clique three-point line (Figure 6.17 and $e$ being in a red matching).

\subsubsection{Three Non-clique Three-point Lines}

We are now interested in the ways that a clique element $e$ can be on three non-clique three-point lines, but be in no $U_{2,4}$-restrictions. Recall from Section 6.4.2 that there are eleven ways that such a clique element can be on two non-clique three-point lines, displayed in Figure 6.24. As such, if we find an element $e$ such that $e$ is in no $U_{2,4}$-restrictions and on three non-clique three-point lines, then any two of the non-clique three-point lines must form one of the eleven configurations.

As it will be useful, we construct Table 6.1 showing how the eleven configurations arise. From this table, for example, we can see that in $\Xi$ (Figure 6.24.5), $e$ lies inside a GG configuration, and is also in a red matching.

Note that although $\Pi$ (Figure 6.24.6) looks like it contains a GG (Figure 6.17.1), it does not. In $\Pi$, there are two GR (Figure 6.17.2) configurations, and $e$ is not on a line with the two green triangles. 


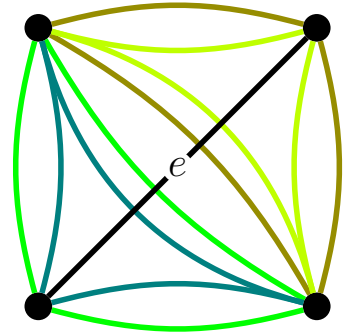

6.24.1: $\Gamma$ ( $\mathrm{GG}$ and $\mathrm{GG}$ )

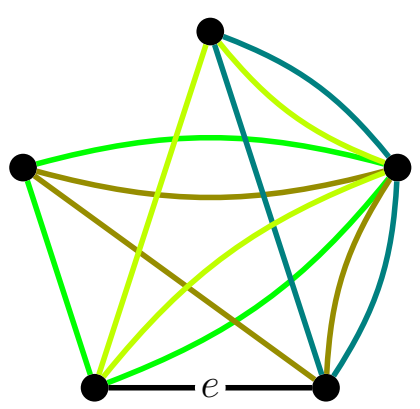

6.24.2: $\Delta$ ( $\mathrm{GG}$ and $\mathrm{GG})$

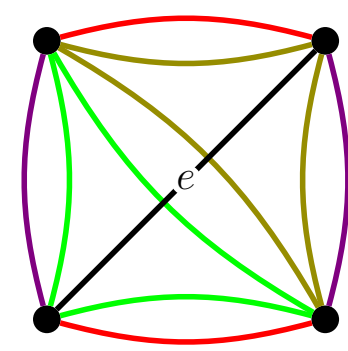

6.24.3: $\Theta$ (GG and RRsquare)

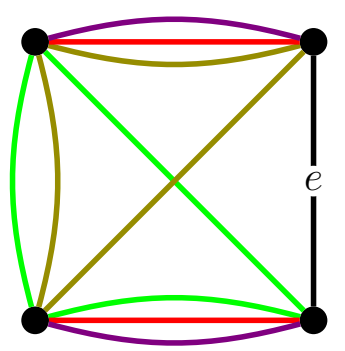

6.24.6: $\Pi \quad(\mathrm{GR}$ and GR) digon)

6.24.5: $\Xi$ (GG and $e$ in a red matching)
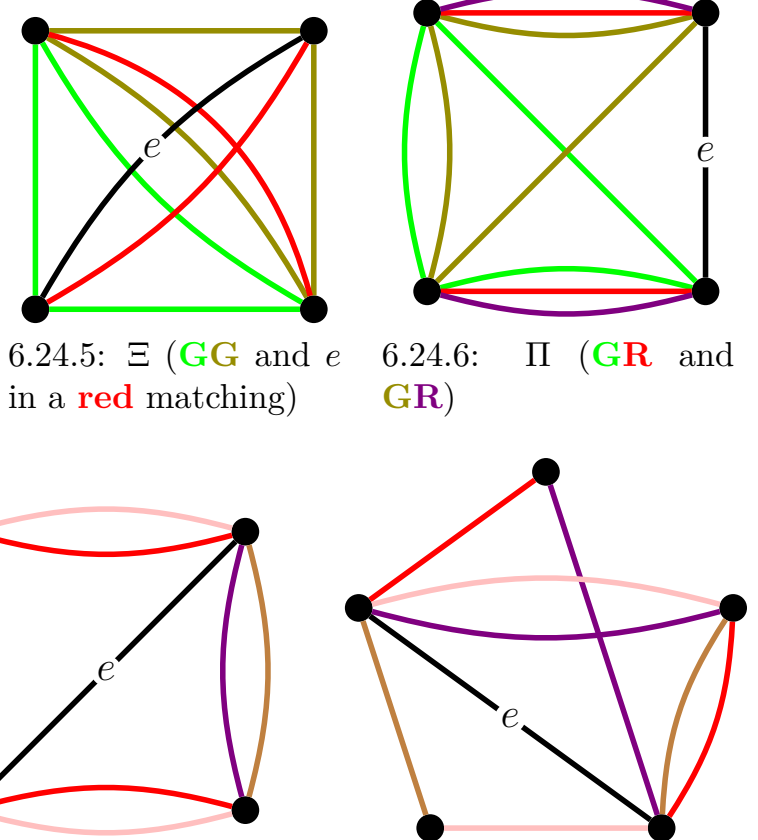

6.24.9: $\Phi$ (RR-square and RR-square)

6.24.7: $\Sigma$ (GR and $e$ in a red matching)

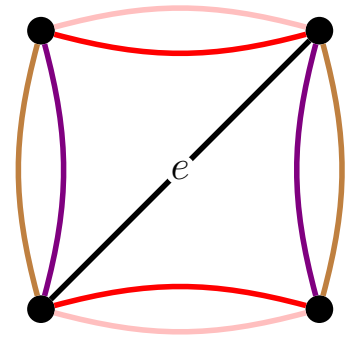

6.24.8: $\Upsilon$ (RR-square and RR-square)

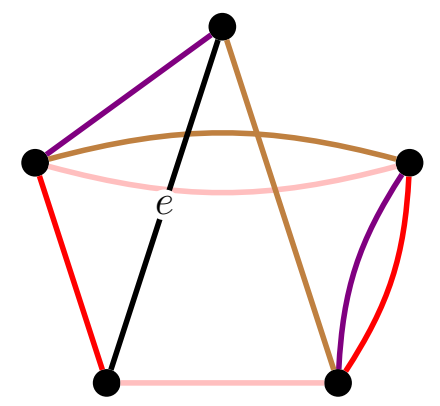

6.24.10: $\Psi$ (RR-digon and RR-digon)

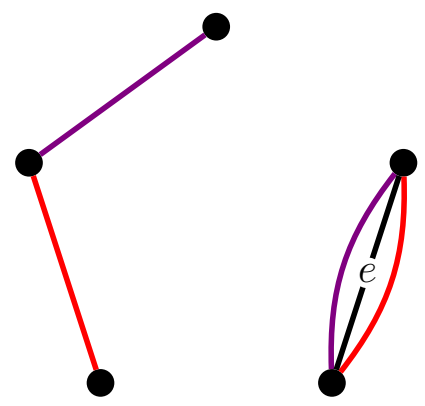

6.24.11: $\Omega$ (e in a red matching and $e$ in a red matching)

Figure 6.24: Two Non-clique Three-point Lines 


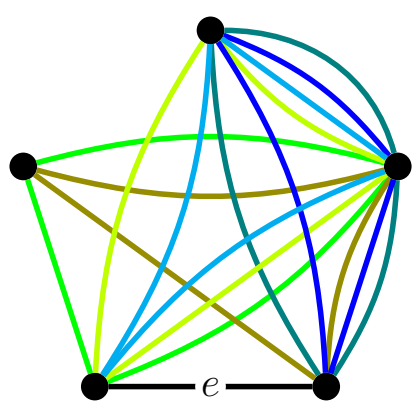

6.25.1: Extra GG

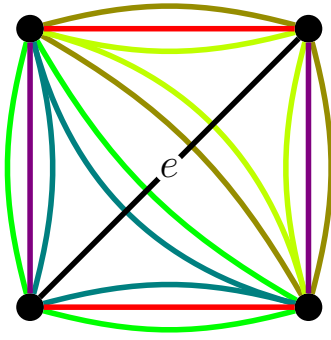

Extra RR-

6.25 .2 : square

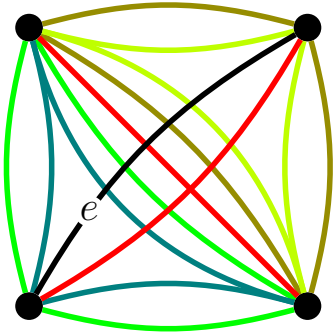

6.25.4: $e$ in an extra red matching

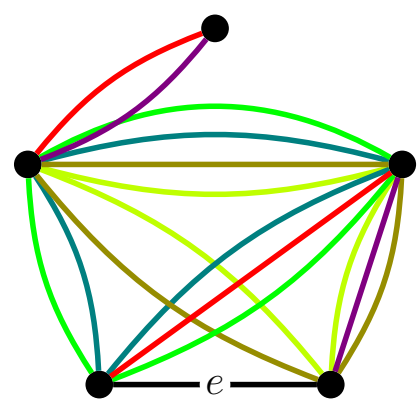

6.25.3: Extra RR-digon

Figure 6.25: Starting from a $\Gamma$

\subsubsection{1 $\Gamma$}

We start by considering the case where the first two lines form a $\Gamma$ (Figure 6.24.1). From Table 6.1, we can see that this particular configuration is constructed from two GG (Figure 6.17.1) three-point lines. Also from Table 6.1, we can instantly dismiss the case where the third non-clique threepoint line is a GR (Figure 6.17.2), as there is no way that a GG and a GR can combine to give two non-clique three-point lines.

Identify a GG inside the $\Gamma$. We are going to add another GG onto this GG. From Table 6.1, we see that there are two ways to do this, either as a $\Gamma$ or a $\Delta$ (Figure 6.24.2). If we choose a $\Gamma$, then we will get a QI (Figure 6.8.1), which is forbidden. Hence we must choose a $\Delta$. It is easier to see this configuration if we start with a $\Delta$ and double-up one of the GGs inside it, giving a $\Gamma$, as shown in Figure 6.25.1. In this case, all_lines $(5,[[0,1,4]$, [0, $1,4],[0,3,6],[0,3,6],[1,2,7],[2,3,9]], 8)$ returns the empty list, so this configuration is forbidden.

The next case is to once again identify a GG inside $\Gamma$, and extend this GG into 
a $\Theta$ (Figure 6.24.3) by adding a RR-square (Figure 6.17.3). This is shown in Figure 6.25.2. In this case, all_lines $(4,[[0,2,4],[0,2,4],[1$, $2,5],[1,2,5],[0,5],[1,4]], 3)$ returns the empty list, so this configuration is forbidden.

The next case is to once again identify a GG inside $\Gamma$, and extend this GG into a $\Lambda$ (Figure 6.24.4) by adding a RR-digon (Figure 6.17.4). This is shown in Figure 6.25.3. In this case, we can see a CC (Figure 6.12.4), so this configuration is forbidden.

The final case is to identify a GG inside $\Gamma$, and extend this GG into a $\Xi$ (Figure 6.24.5) by making $e$ part of a red matching. This is shown in Figure 6.25.4. In this case, all_lines $(4,[[0,2,4],[0,2,4],[1,2$, $5],[1,2,5],[2,3]], 3)$ returns the empty list, hence this configuration is forbidden.

\subsubsection{2 $\Delta$}

We now consider the case where the first two lines form a $\Delta$ (Figure 6.24.1). From Table 6.1, we can see that this configuration is constructed from two GG (Figure 6.17.1) three-point lines. Also from Table 6.1, we can instantly dismiss the case where the third non-clique three-point line is a GR (Figure 6.17.2), as there is no way that a GG and a GR can combine to give two non-clique three-point lines.

Identify a GG inside the $\Delta$. We are going to add another GG onto this GG. From Table 6.1, we see that there are two ways to do this, either as a $\Gamma$ or a $\Delta$ (Figure 6.24.2). If we choose a $\Gamma$, then we are in a previous case. Hence we must choose a $\Delta$. If we remain on five vertices, then it is possible to add in an extra GG in a $\Delta$ configuration with both existing GGs, as shown in Figure 6.26.1. However, this configuration contains an OM (Figure 6.8.2), and so is forbidden. We now try to add in a third GG while using six vertices. Note that GG is determined by an edge of the complete graph vertex-disjoint from $e$. This edge must touch the sixth vertex. If this edge does not touch a particular existing green triangle, then we can find a TL (Figure 6.8.3), which is forbidden. Hence this edge must touch all existing green triangles, and there is only one place it could go, shown in Figure 6.26.2. In this case, all_lines $(6,[[0,1,5],[0,2,6],[0,4,8],[1,3$, 


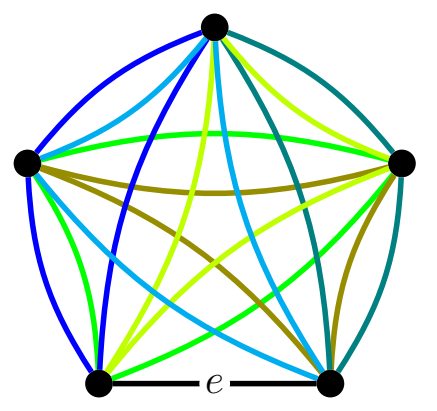

6.26.1: Extra GG I

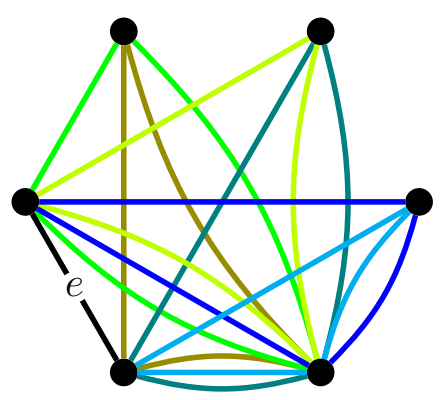

6.26.2: Extra GG II

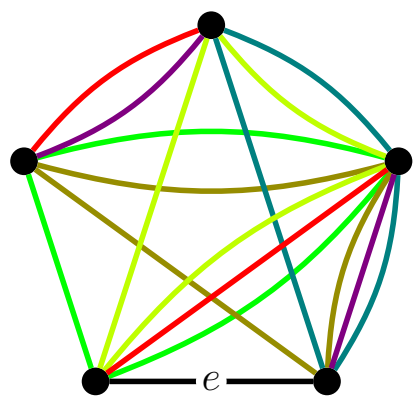

6.26.3: Extra RR-digon

Figure 6.26: Starting from a $\Delta$

$10],[2,3,12],[3,4,14]], 7)$ outputs that $e$ can be on three nonclique three-point lines. We call this the line-star configuration, shown in Figure 6.34.1.

The next case is to once again identify a GG inside $\Delta$, and extend this GG into a $\Theta$ (Figure 6.24.3) by adding a RR-square (Figure 6.17.3). However we place the two red matchings, we are unable to make every red matching edge touch every green triangle, so this case is a contradiction to Lemma 6.3.4.

The next case is to once again identify a GG inside $\Delta$, and extend this GG into a $\Lambda$ (Figure 6.24.4) by adding a RR-digon (Figure 6.17.4). The path component of the digon must touch the vertex that every existing green triangle is incident with, as otherwise we would have a contradiction to Lemma 6.3.4. The parallel component must also touch all four existing green triangles, and hence there is only one place it could go, regardless of the number of vertices. This configuration, shown in Figure 6.26.3, contains a PM (Figure 6.12.3), and so is forbidden.

The final case is to identify a GG inside $\Gamma$, and extend this GG into a $\Xi$ (Figure 6.24.5) by making $e$ part of a red matching. There is only one way to 


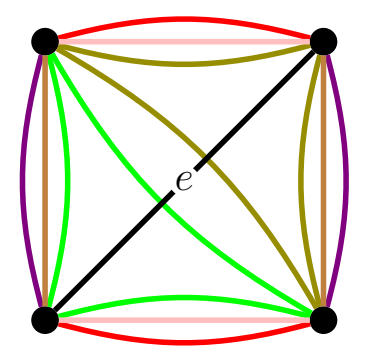

Figure 6.27: Starting from a $\Theta$

do this, and it fails to turn the other GG into a $\Xi$, so this case is discarded.

\subsubsection{3 $\Theta$}

We now consider the case where the first two lines form a $\Theta$ (Figure 6.24.3). From Table 6.1, we can see that this configuration is constructed from a GG (Figure 6.17.1) three-point line and a RR-square (Figure 6.17.3) three-point line. Also from Table 6.1, we note that the only case we need to consider is the case where the third non-clique three-point line is a RR-square, as all other possibilities either do not occur or have already been considered. In particular, we do not need to consider adding a GG (Figure 6.17.1), as then the two GGs would have to form either a $\Gamma$ (Figure 6.24.1) or $\Delta$ (Figure 6.24.2), which we have already considered.

From Table 6.1, we see that we can add in a RR-square so that it is either in a $\Upsilon$ (Figure 6.24.8) or a $\Phi$ (Figure 6.24.9) with the existing RR-square. However, it is not possible to have a $\Phi$, as this requires five vertices, and such a configuration would not have each of the RR-squares in a $\theta$ (Figure 6.24.3) with the existing GG, so cannot occur. Hence there is only one configuration possible, shown in Figure 6.27. In this case, all_lines (4, [ [0, $2,4],[1,2,5],[0,5],[0,5],[1,4],[1,4]], 3)$ outputs the empty list, so this case is forbidden.

\subsubsection{4 $\Lambda$}

We now consider the case where the first two lines form a $\Lambda$ (Figure 6.24.4). From Table 6.1, we can see that this configuration is constructed from a GG (Figure 6.17.1) three-point line and a RR-digon (Figure 6.17.4) three-point 


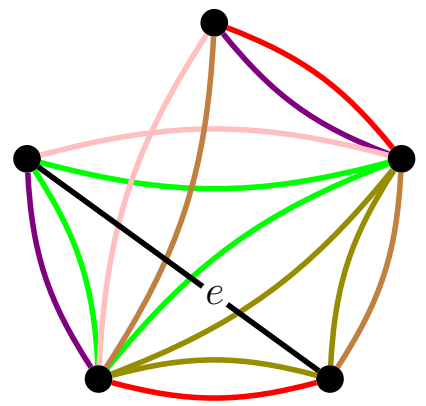

Figure 6.28: Starting from a $\Lambda$

line. Also from Table 6.1, we note that the only case we need to consider is the case where the third non-clique three-point line is a RR-digon, as all other possibilities either do not occur or have already been considered. In particular, we do not need to consider adding a GG (Figure 6.17.1), as then the two GGs would have to form either a $\Gamma$ (Figure 6.24.1) or $\Delta$ (Figure 6.24.2), which we have already considered.

From Table 6.1, we see that we must add on the second RR-digon in a $\Psi$ (Figure 6.24.10) configuration with the existing RR-digon. There is only one way to do this, shown in Figure 6.28. In this case, we can find a HN (Figure 6.13.1), so this case is forbidden.

\subsubsection{5 $\Xi$}

We now consider the case where the first two lines form a $\Xi$ (Figure 6.24.5). From Table 6.1, we can see that this configuration is constructed from a GG (Figure 6.17.1) three-point line with the second three-point line coming from $e$ being in a red matching. Also from Table 6.1, we note that the only case we need to consider is the case where the third non-clique three-point line arises from $e$ being on a red matching, as all other possibilities either do not occur or have already been considered. Note that we cannot combine a red matching containing $e$ with a GR, as then the GR and the GG would have to combine to form two non-clique three-point lines containing $e$, which by Table 6.1 we see does not happen.

From Table 6.1, we see that we put $e$ into a second red matching in a $\Omega$ (Figure 6.24.11) configuration with the existing red matching containing $e$. This requires five vertices, and such a configuration would not have each of 


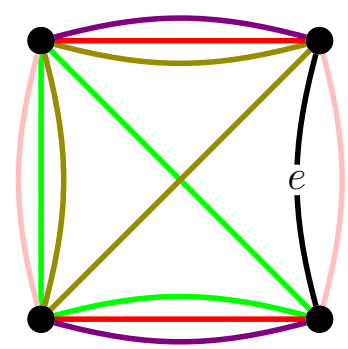

Figure 6.29: Starting from a $\Pi$

the red matchings containing $e$ in a $\Xi$ configuration with the GG, and so this cannot occur.

\subsubsection{6 $\Pi$}

We now consider the case where the first two lines form a $\Pi$ (Figure 6.24.6). From Table 6.1, we can see that this configuration is constructed from two GR (Figure 6.17.2) three-point lines. Also from Table 6.1, we note that the only cases we need to consider are the case where the third non-clique three-point line arises from a GR, and the case where the third non-clique three-point line arises from $e$ being in a red matching as all other possibilities do not occur.

Identify a GR inside the $\Pi$. We are going to add another GR onto this GR. From Table 6.1, we see that there is one way to do this, as a $\Pi$. However, this will not add the third GR into a $\Pi$ with the second GR, as is required. Hence this case is forbidden.

The final case is to identify a GR inside $\Pi$, and extend this GR into a $\Sigma$ (Figure 6.24.7) by making $e$ part of a red matching. This is shown in Figure 6.29. In this case, all_lines $(4,[[0,1,3],[0,2,4],[0,5]$, $[1,4],[1,4]], 5)$ outputs that $e$ is on three non-clique three-point lines. We call this the Betsy Ross configuration, shown in Figure 6.34.2.

\subsubsection{7 $\Sigma$}

We now consider the case where the first two lines form a $\Sigma$ (Figure 6.24.7). From Table 6.1, we can see that this particular configuration is constructed from a GR (Figure 6.17.2) three-point line with the second non-clique three- 


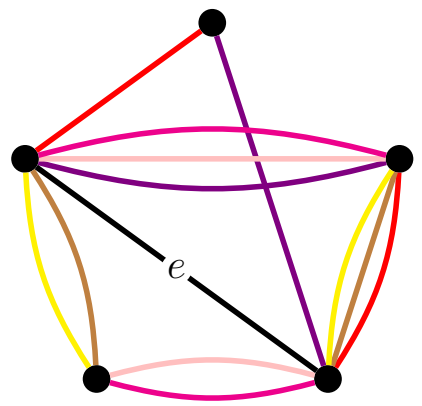

Figure 6.30: Starting from a $\Upsilon$

point line coming from $e$ being in a red matching. Also from Table 6.1, we note that the only case we need to consider is the case where the third non-clique three-point line arises from $e$ being in a red matching as all other possibilities either do not occur or have already been considered.

From Table 6.1, we see that we put $e$ into a second red matching in a $\Omega$ (Figure 6.24.11) configuration with the existing red matching containing $e$. This requires five vertices, and such a configuration would not have each of the red matchings containing $e$ in a $\Sigma$ configuration with the GR, and so this cannot occur.

\subsubsection{8 $\Upsilon$}

We now consider the case where the first two lines form a $\Upsilon$ (Figure 6.24.8). From Table 6.1, we can see that this particular configuration is constructed from two RR-square (Figure 6.17.3) three-point lines. Also from Table 6.1, we note that the only case we need to consider is the case where the third non-clique three-point line arises from a RR-square as all other possibilities either do not occur or have already been considered.

Identify a RR-square inside the $\Upsilon$. We are going to add another RR-square onto this RR-square. From Table 6.1, we see that there are two ways to do this, either as a $\Upsilon$ or a $\Phi$ (Figure 6.24.9). If we choose a $\Upsilon$, then we will get a TM (Figure 6.10.2), which is forbidden. Hence we must choose a $\Phi$. It is easier to see this configuration if we start with a $\Phi$ and doubleup one of the RR-squares inside it, giving a $\Upsilon$, as shown in Figure 6.30. In this case, all_lines $(5,[[1,5],[1,9],[1,9],[2,4],[2,8]$, $[2,8]], 7)$ returns the empty list, so this configuration is forbidden. 


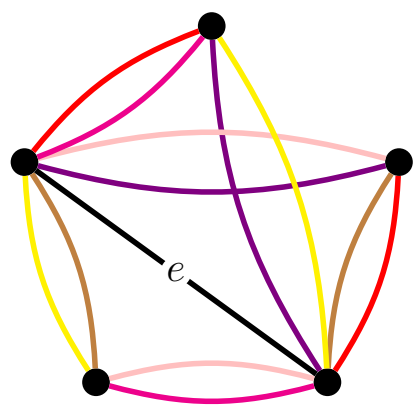

6.31.1: Five vertices

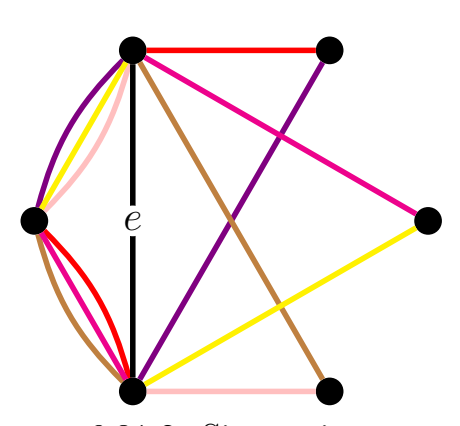

6.31.2: Six vertices

Figure 6.31: Starting from a $\Phi$

\subsubsection{9 $\Phi$}

We now consider the case where the first two lines form a $\Phi$ (Figure 6.24.9). From Table 6.1, we can see that this particular configuration is constructed from two RR-square (Figure 6.17.3) three-point lines. Also from Table 6.1, we note that the only case we need to consider is the case where the third non-clique three-point line arises from a RR-square as all other possibilities either do not occur or have already been considered.

Furthermore, we see from Table 6.1 that there are two ways to add in the third RR-square relative to each existing RR-square: $\Upsilon$ (Figure 6.24.8), which has already been covered, and $\Phi$. So any two RR-squares in our final configuration must form a $\Phi$.

We start by attempting to create such a configuration on five vertices. There are three choices for a vertex for our third RR-square to avoid, and two of these vertices would lead to a $\Upsilon$. Hence there is only one place for the third RR-square, shown in Figure 6.31.1. In this case, we can find a BC (Figure 6.10.3), and therefore this case is forbidden.

We now try to construct such a configuration on six vertices. In $\Phi$, the two RR-squares have two edges that are adjacent in both RR-squares. As such, there is only one place that we can put the third RR-square without ruining this condition for one of the existing RR-squares. This configuration is shown in Figure 6.31.2. In this case, all_lines $(6,[00,14],[4,7],[5,14]$, $[6,14],[7,11],[7,13]], 8)$ outputs that $e$ is on three non-clique three-point lines. We call this the two-parallel configuration, shown in Figure 6.34.3. 


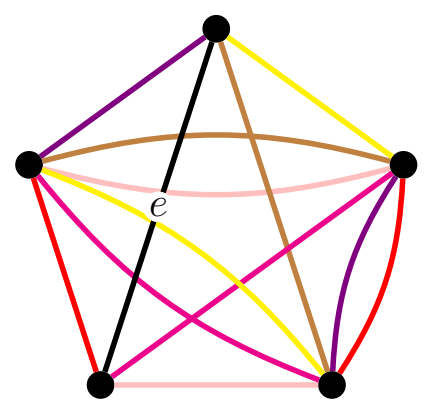

Figure 6.32: Starting from a $\Psi$

\subsubsection{0 $\Psi$}

We now consider the case where the first two lines form a $\Psi$ (Figure 6.24.10). From Table 6.1, we can see that this particular configuration is constructed from two RR-digon (Figure 6.17.4) three-point lines. Also from Table 6.1, we note that the only case we need to consider is the case where the third non-clique three-point line arises from a RR-digon as all other possibilities either do not occur or have already been considered.

We also note from Table 6.1 that there is only way to add in the third RRdigon, namely that any two of the RR-digons must form a $\Psi$. In light of this, there is only one way to add in the third RR-digon, shown in Figure 6.32. In this case, we can find a XS (Figure 6.10.1), so this case is forbidden.

\subsubsection{1 $\Omega$}

Finally, we are in the case where the first two lines form a $\Omega$. From Table 6.1, we can see that this configuration is formed by having $e$ on two red matchings. Also, we note that the third non-clique three-point line must also be made by having $e$ on a red matching, as all other possibilities either do not occur or have already been considered. Furthermore, any two red matchings in our final configuration must form a $\Omega$.

We start by constructing this on five vertices. Taking into account the previously mentioned restriction, there is only one place that we could place the third red matching, which is in such a way that an AE (Figure 6.11.2) configuration results, which is forbidden.

We now construct this on six vertices. Taking into account the previously 


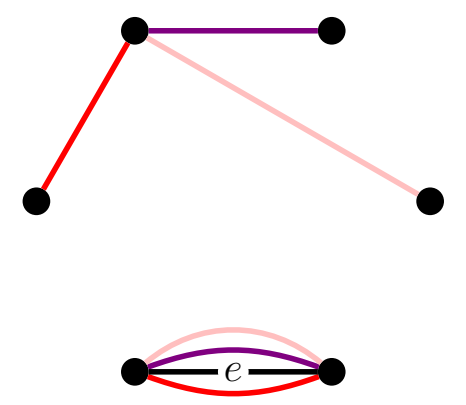

Figure 6.33: Starting from a $\Omega$

mentioned restriction, there is only one place that we could place the third red matching, shown in Figure 6.33. In this case, all_lines $(6,[0,11]$, $[0,13],[0,14]], 0)$ outputs that $e$ is on three non-clique three-point lines. We call this the matching-star configuration, shown in Figure 6.34.4.

\subsubsection{Summary}

To summarise, there are four ways to have a clique element $e$ on three nonclique three-point lines, yet no $U_{2,4}$-restrictions. These are shown in Figure 6.34.

The line-star configuration consists of a highlighted edge $e$ and a star that is vertex-disjoint from $e$. Each edge of the star is contained in two green triangles, one using each of the end vertices of $e$. A schematic is given in Figure 6.44.

The Betsy Ross configuration consists of two green triangles and three red triangles on $K_{4}$. If we label the vertices of $K_{4}$ such that $e$ is the line $a b$, then the two green triangles are $a c d$ and $a b d$, while the three red matchings are $(a c, b d)$ twice and $(a b, c d)$.

The two-parallel configuration consists of three distinguished vertices, two of which are the ends of $e$, and a collection of other vertices. For each nondistinguished vertex, a RR-square not containing $e$ is formed that uses the three distinguished vertices.

The matching-star configuration consists of a distinguished edge $e$ and a star that is vertex-disjoint from $e$. For each edge of this star, form a matching that uses this edge and $e$. 


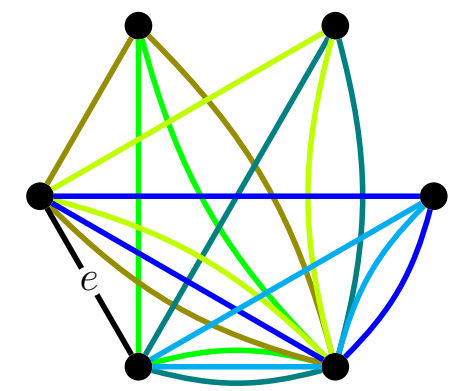

6.34.1: Line-star configuration

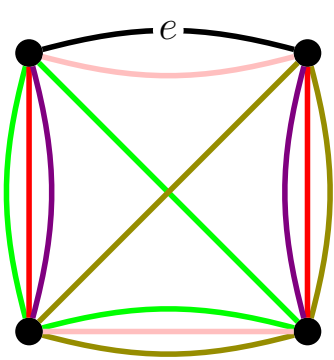

6.34.2: Betsy Ross configuration
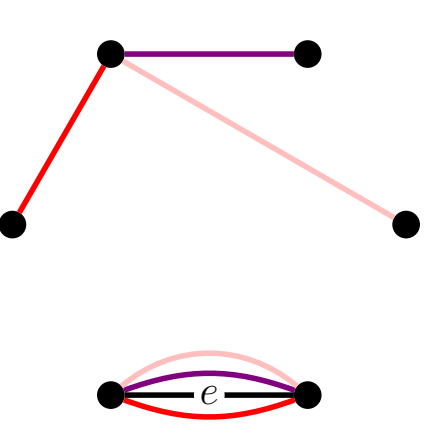

6.34.4: Matching-star configuration

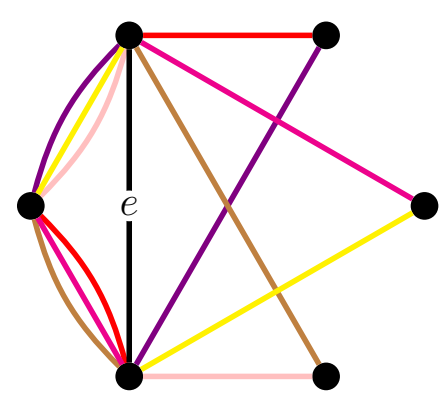

6.34.3: Two-parallel configuration

Figure 6.34: Three non-clique three-point lines

\section{$6.5 U_{2,4}$-restrictions}

We now analyse how an augmented clique can give rise to an $U_{2,4}$-restriction containing at least one element of the clique. Hence an $U_{2,4}$-restriction must contain one, two, or three non-clique elements. We consider each case in turn.

\subsubsection{One Non-clique Element}

We consider the case that a $U_{2,4}$-restriction contains exactly one non-clique element. In this case, the $U_{2,4}$-restriction contains a triangle of the clique. There are two ways to add in one non-clique element: a red matching or a green triangle. The red matching does not create any $U_{2,4}$-restrictions, while the green triangle creates one. 


\subsubsection{Two Non-clique Elements}

We consider the case that a $U_{2,4}$-restriction contains exactly two non-clique elements. If we have a $U_{2,4}$-restriction containing exactly two non-clique elements, then we can find a $U_{2,3}$-restriction containing one clique element and two non-clique elements. In Sections 6.4.1.2 - 6.4.1.4, we looked for long lines that contained two non-clique elements. The result of this analysis was that the only way to obtain a $U_{2,4}$-restriction with exactly two non-clique elements is the doubled-up red matching (Figure 6.15.2).

\subsubsection{Three Non-clique Elements}

We consider the case that a $U_{2,4}$-restriction contains exactly three non-clique elements. There are four different cases to consider, and we go through them in a methodical way.

\subsubsection{Three Green Triangles}

From Section 6.4.1.2, we know that the first two green triangles meet at a triangle, an edge, or a vertex, so we only need to consider how to place the third triangle.

We start with the first two triangles meeting at a triangle (Figure 6.14.1). We cannot use only these three vertices, as that would create a QI (Figure 6.8.1), which is forbidden. If we use one extra vertex, the only way to place the third green triangle is as in $\mathrm{GGG}_{1}$ (Figure 6.35.1). If we use two extra vertices, we get $\mathrm{GGG}_{2}$ (Figure 6.35.2). We cannot use any more vertices, as this would lead to vertex-disjoint green triangles, which is forbidden by TL (Figure 6.8.3).

Now we start with the first two triangles meeting at an edge (Figure 6.14.2). When we add in the third green triangle, we cannot add in it on top of an existing triangle, as this case has already been considered. With this in mind, there is only one way to place the third green triangle if we use only the existing four vertices, $\mathrm{GGG}_{3}$ (Figure 6.35.3).

With five vertices, the third green triangle is determined by an edge in the span of the first two triangles. There are three such edges - the edge that 


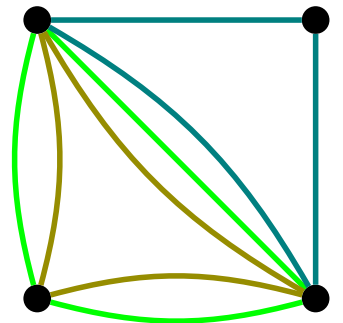

6.35.1: $\mathrm{GGG}_{1}$

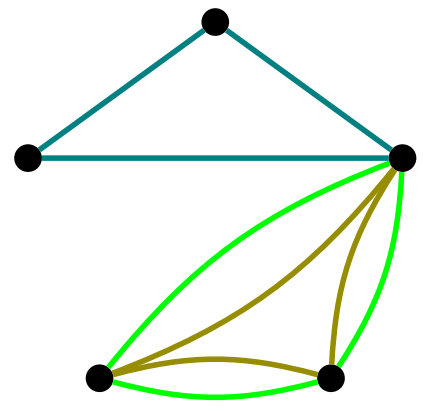

6.35.2: $\mathrm{GGG}_{2}$

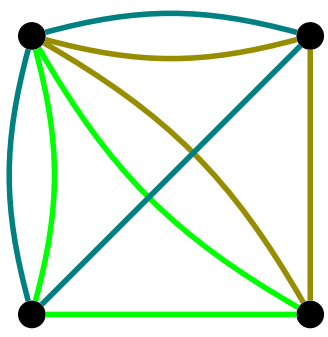

6.35.3: $\mathrm{GGG}_{3}$

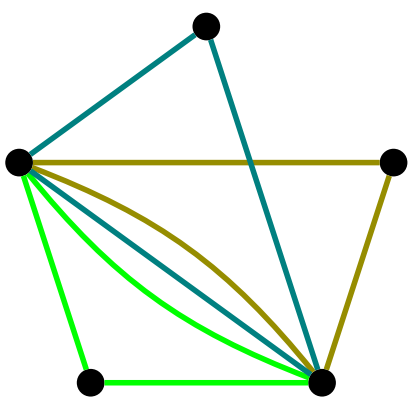

6.35.4: $\mathrm{GGG}_{4}$

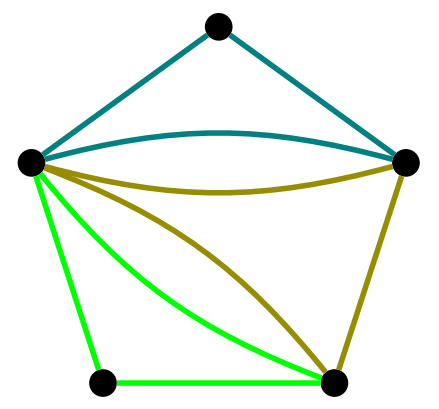

6.35.6: $\mathrm{GGG}_{6}$

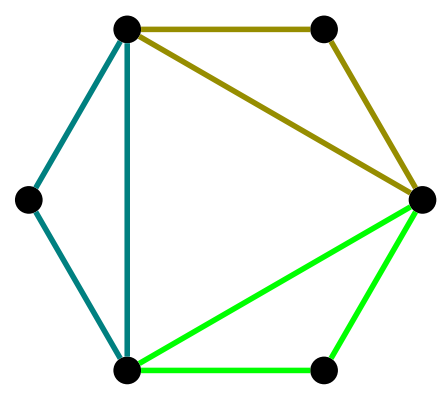

6.35.8: $\mathrm{GGG}_{8}$

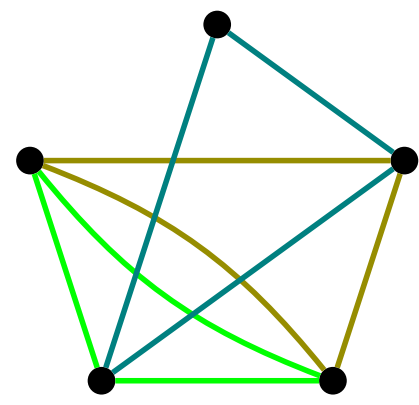

6.35.5: $\mathrm{GGG}_{5}$

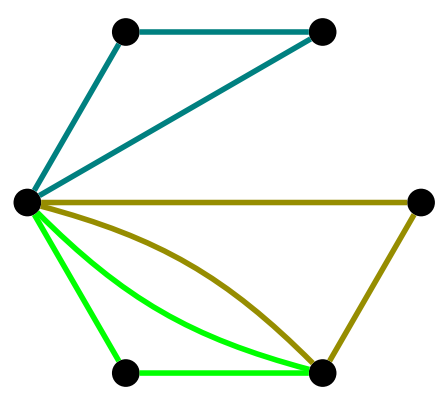

6.35.7: $\mathrm{GGG}_{7}$

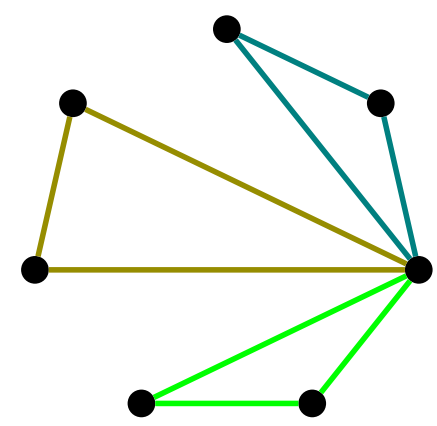

6.35.9: GGG9

Figure 6.35: Three Green Triangles 
is in both existing triangles $\left(\mathrm{GGG}_{4}\right.$, Figure 6.35.4), the edge that is in no existing triangles $\left(\mathrm{GGG}_{5}\right.$, Figure 6.35.5), and the edge that is in exactly one of the existing triangles $\left(\mathrm{GGG}_{6}\right.$, Figure 6.35.6).

With six vertices, the third triangle is determined by an existing vertex. This existing vertex must touch both existing triangles (because of TL, Figure 6.8.3), so there is only one place to put it, giving $\mathrm{GGG}_{7}$ (Figure 6.35.7).

Finally, we start with the first two triangles meeting at a vertex (Figure 6.14.3). The final green triangle must meet both existing triangles at a vertex, and not use any existing edges, or we are in a previously considered case. There is no way to do this with no extra vertices. With one extra vertex, there is only one way, $\mathrm{GGG}_{8}$ (Figure 6.35.8). With two extra vertices, there is also only one to do this, $\mathrm{GGG}_{9}$ (Figure 6.35.9).

We now go through each configuration in turn and check for the existence of $U_{2,4}$-restrictions containing all three of the extension elements. These checks are done by the lines() function from Appendix 6.A.4. The function calls, and an interpretation of the output are given below.

$\mathrm{GGG}_{1}$ lines(4, [ [0, 2, 4], [0, 2, 4], [1, 2, 5]]) outputs that there is no line containing the three extension elements and a clique element.

$\mathrm{GGG}_{2}$ lines $(5,[[0,2,5],[1,3,8],[1,3,8]])$ outputs that there is no line containing the three extension elements and a clique element.

$\mathrm{GGG}_{3}$ lines $(4,[[0,1,3],[0,2,4],[1,2,5]])$ outputs that there is no line containing the three extension elements and a clique element.

$\mathrm{GGG}_{4}$ lines(5, [[1, 2, 7], [4, 5, 7], [7, 8, 9] ]) outputs that there is no line containing the three extension elements and a clique element.

$\mathrm{GGG}_{5}$ This is OM (Figure 6.8.2), and so is forbidden.

$\mathrm{GGG}_{6}$ lines(5, $\left.[[0,2,5],[1,2,7],[7,8,9]]\right)$ outputs that there is no line containing the three extension elements and a clique element.

$\mathrm{GGG}_{7}$ lines(6, $\left.[[0,3,7],[1,3,10],[12,13,14]]\right)$ outputs that there is no line containing the three extension elements and a 
clique element.

$\mathrm{GGG}_{8}$ This is TB (Figure 6.8.4), and so is forbidden.

$\mathrm{GGG}_{9}$ This is RA (Figure 6.8.6), and so is forbidden.

So there are no configurations of interest.

\subsubsection{Two Green Triangles and One Red Matching}

From Section 6.4.1.2, we know that the first two green triangles meet at a triangle, an edge, or a vertex, so we only need to consider how to place the matching.

First note that we cannot add more than two extra vertices, as if we added three extra vertices, then one edge of the matching must be vertex-disjoint from the existing green triangles, contradicting Lemma 6.3.4.

We start with the two green triangles meeting at a triangle (Figure 6.14.1). We cannot use three vertices, as a red matching requires four vertices. With one extra vertex, the only thing we can do is $\mathrm{GGR}_{1}$ (Figure 6.36.1). With two extra vertices, we cannot have a matching edge go between these vertices, as that would contradict Lemma 6.3.4. This leaves only GGR 2 (Figure 6.36.2). Now we start with the two green triangles meeting at an edge (Figure 6.14.2). If we stay on four vertices, there are two places where a red matching could go - either both edges in parallel with an existing triangle edge $\left(G_{G R}\right.$, Figure 6.36.3), or using the edge that the existing triangles avoid $\left(\mathrm{GGR}_{4}\right.$, Figure 6.36.4). We now add in one extra vertex. Due to Lemma 6.3.4, the first edge of the matching must go to one of the vertices that both existing green triangles touch. This leaves two places that the final matching edge can go - either in parallel with one of the triangle edges $\left(\mathrm{GGR}_{5}\right.$, Figure 6.36.5), or incident with the two vertices that are incident with exactly one green triangle $\left(\mathrm{GGR}_{6}\right.$, Figure 6.36.6). With two extra vertices, the restrictions already discussed only allow one configuration, GGR $_{7}$ (Figure 6.36.7).

Finally, we start with the two green triangles meeting at a vertex (Figure 6.14.3). Starting with the existing five vertices, the first edge placed of the matching could either be in parallel with a triangle edge, or it could not. If it is in parallel, and taking Lemma 6.3.4 into consideration, there is only one place for it to go, namely it must be incident with both green 


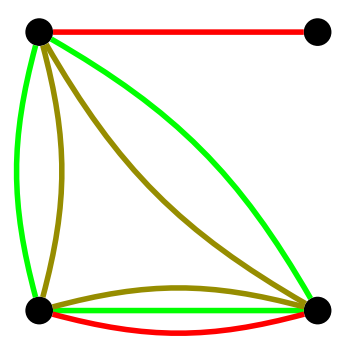

6.36.1: $\mathrm{GGR}_{1}$

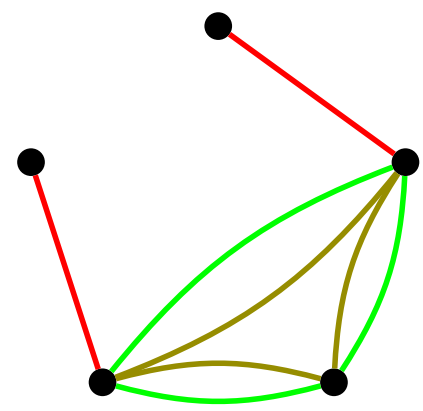

6.36.2: GGR

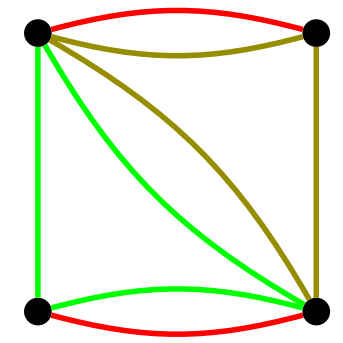

6.36.3: $\mathrm{GGR}_{3}$

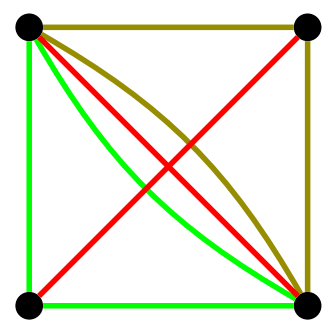

6.36.4: $\mathrm{GGR}_{4}$

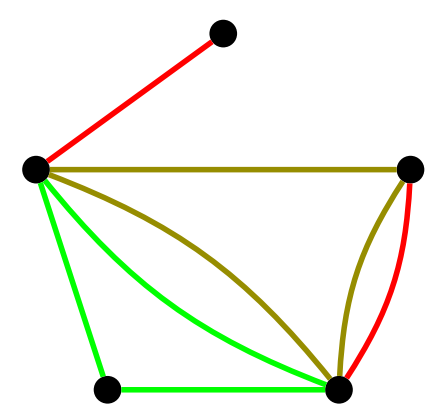

6.36.5: GGR

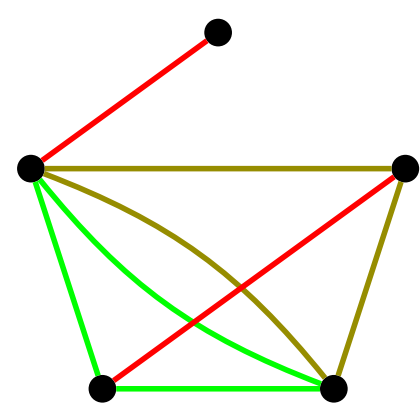

6.36.6: GGR 6

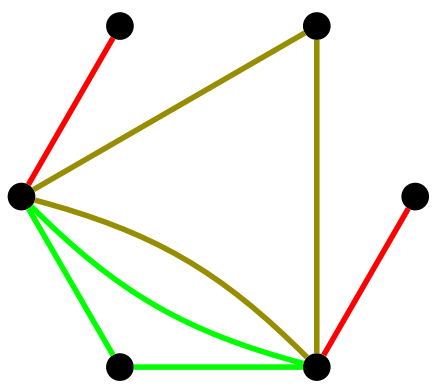

6.36.7: $\mathrm{GGR}_{7}$

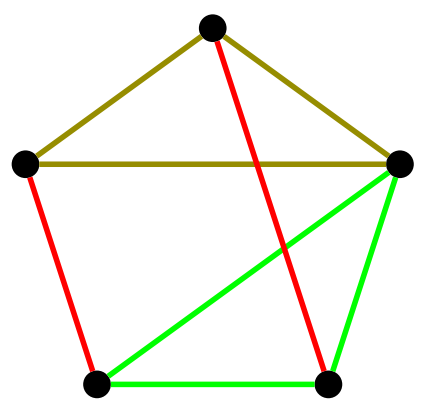

6.36.9: GGR $_{9}$

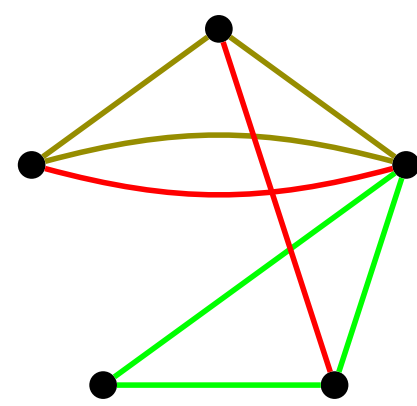

6.36.8: $\mathrm{GGR}_{8}$

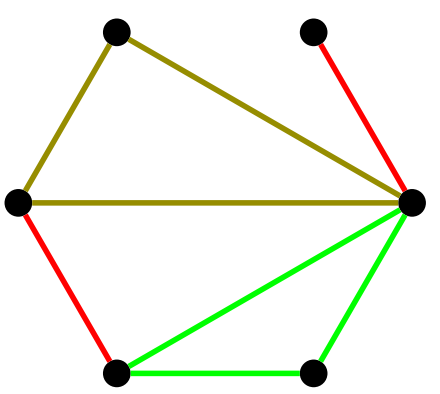

6.36.10: GGR $\mathbf{R}_{10}$

Figure 6.36: Two Green Triangles and One Red Matching 
triangles. Taking Lemma 6.3.4 into consideration again, there is also only one place for the second matching edge to go, giving GGR (Figure 6.36.8). If neither edge is in parallel, there are only four vertices that our matching could span, forcing us to have $\mathrm{GGR}_{9}$ (Figure 6.36.9).

Adding in extra vertices, as every matching edge that uses these new vertices must touch both triangles, and there is only one vertex that both triangles touch, we can only add one extra vertex. Once this vertex, and the matching edge required by it, are added, there is only one place the last matching edge could go, giving GGR $_{10}$ (Figure 6.36.10).

We now go through each configuration in turn and check for the existence of $U_{2,4}$-restrictions containing all three of the extension elements. These checks are done by the lines () function from Appendix 6.A.4. The function calls, and an interpretation of the output are given below.

$\operatorname{GGR}_{1}$ lines $(4,[[0,2,4],[0,2,4],[1,4]])$ outputs that there is no line containing the three extension elements and a clique element.

$\mathrm{GGR}_{2}$ This is CC (Figure 6.12.4), and so is forbidden.

$\operatorname{GGR}_{3}$ lines $(4,[[0,2,4],[1,2,5],[1,4]])$ outputs that $[a, b$, c, 3] is a line containing the three extension elements. This is shown in Figure 6.43.1.

$\mathrm{GGR}_{4}$ lines $(4,[[0,2,4],[1,2,5],[2,3]])$ outputs that there is no line containing the three extension elements and a clique element.

$\mathrm{GGR}_{5}$ lines $(5,[[1,2,7],[7,8,9],[1,5]])$ outputs that there is no line containing the three extension elements and a clique element.

$\mathrm{GGR}_{6}$ This is KF (Figure 6.12.2), and so is forbidden.

$\mathrm{GGR}_{7}$ lines $(6,[[0,3,7],[2,3,12],[1,14]])$ outputs that there is no line containing the three extension elements and a clique element.

$\mathrm{GGR}_{8}$ This is PM (Figure 6.12.3), and so is forbidden.

$\mathrm{GGR}_{9}$ lines $(5,[[0,2,5],[1,3,8],[4,9]])$ outputs that there is no line containing the three extension elements and a clique element.

$\mathrm{GGR}_{10}$ This is SR (Figure 6.13.5), and so is forbidden.

So $\mathrm{GGR}_{3}$ is the only configuration of interest. 


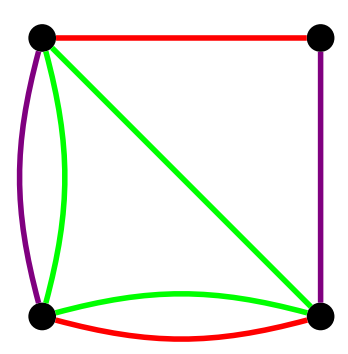

6.37.1: $\mathbf{G R R}_{1}$

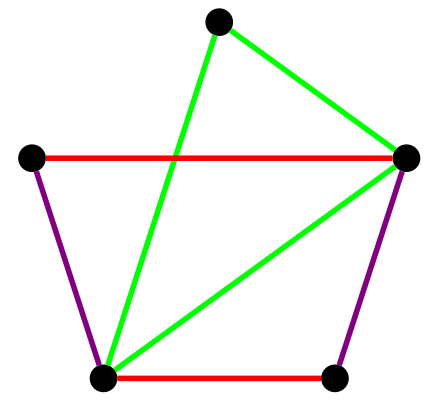

6.37.2: $\mathrm{GRR}_{2}$

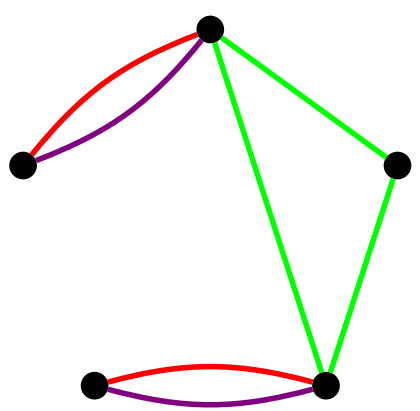

6.37.4: $\mathrm{GRR}_{4}$

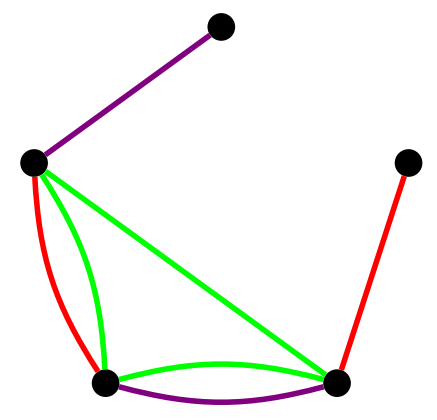

6.37.5: $\mathrm{GRR}_{5}$

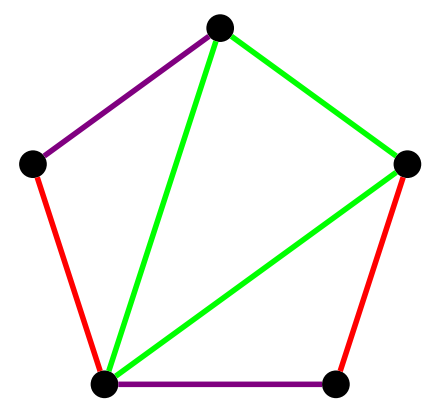

6.37.6: $\mathrm{GRR}_{6}$

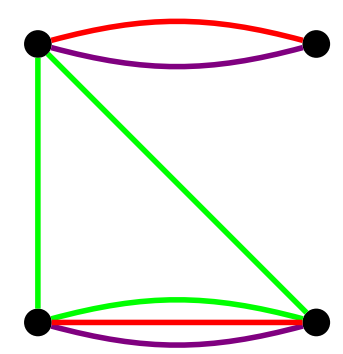

6.37.3: $\mathrm{GRR}_{3}$

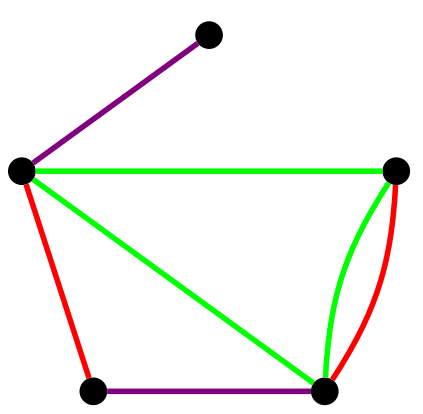

6.37.7: $\mathrm{GRR}_{7}$

Figure 6.37: One Green Triangle and Two Red Matchings I 


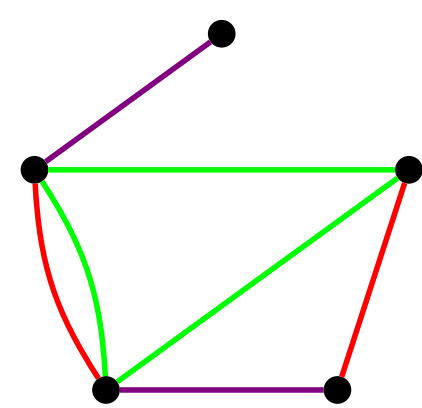

6.38.1: $\mathrm{GRR}_{8}$

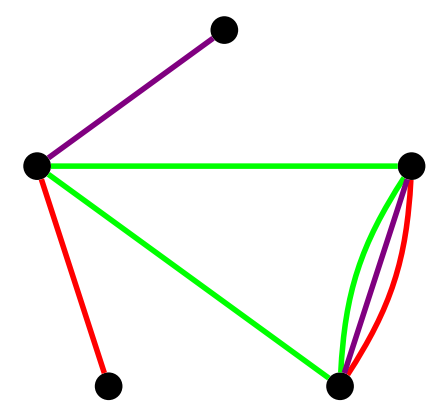

6.38.3: $\mathbf{G R R}_{10}$

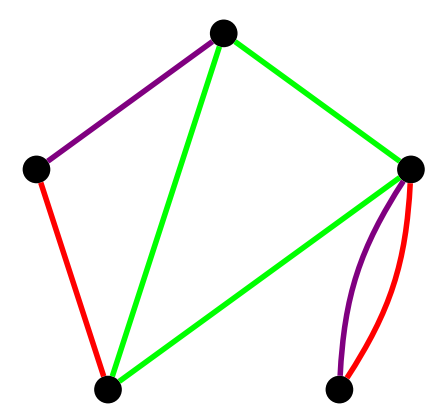

6.38.5: GRR $_{12}$

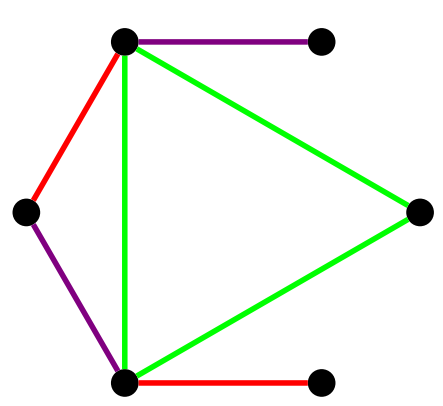

6.38.2: GRR $_{9}$

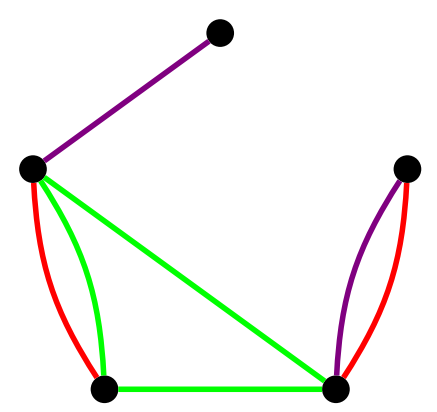

6.38.4: GRR $_{11}$

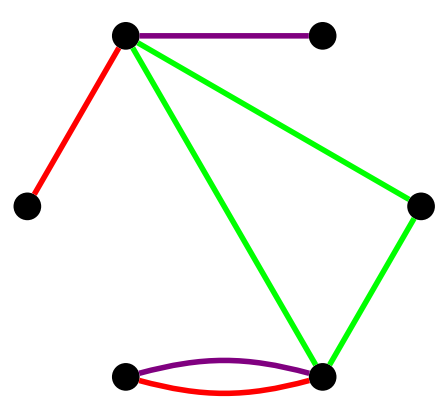

6.38.6: $\mathrm{GRR}_{13}$

Figure 6.38: One Green Triangle and Two Red Matchings II 


\subsubsection{One Green Triangle and Two Red Matchings}

From Section 6.4.1.3 we know that the first two red matchings must form a square, a double, a join, or a parallel. We place the green triangle on each of these in all possible ways.

First note that if we were to add in two additional vertices, we would need to find a vertex that all four matching edges touch because of Lemma 6.3.4. This can never happen, so in this entire case, we can never add more than one vertex.

When we start with the square (Figure 6.15.1), we place the green triangle without adding any additional vertices. There is only one way to do this, $\mathrm{GRR}_{1}$ (Figure 6.37.1). With one additional vertex, the edge that defines the green triangle must touch all four existing matching edges because of Lemma 6.3.4, and $\mathrm{GRR}_{2}$ (Figure 6.37.2) is the only way to do this.

Our next case is to start with the double (Figure 6.15.2). First, we place the green triangle without any additional vertices. There is only one way to do this, $\mathrm{GRR}_{3}$ (Figure 6.37.3). With one additional vertex, the edge that defines the green triangle must touch all four existing matching edges because of Lemma 6.3.4, and $\mathrm{GRR}_{4}$ (Figure 6.37.4) is the only way to do this.

We now start with the join (Figure 6.15.3) and try to add in a green triangle while remaining on five vertices. To do this, we identify an edge in the span of the two existing matchings and place the triangle disjoint from this edge. The edge cannot be parallel to a matching edge, as then that matching edge would be disjoint from the green triangle, a contradiction to Lemma 6.3.4. This leaves four places for the avoided edge. First, we could pick the edge that would turn the path of matchings into a cycle, this gives us $\mathrm{GRR}_{5}$ (Figure 6.37.5). Second, we could pick the edge that does not touch either end of the path of matchings, giving us $\mathrm{GRR}_{6}$ (Figure 6.37.6). Third, we could pick the edge that goes from one end of the path of matchings to the centre of the path, giving us $\mathrm{GRR}_{7}$ (Figure 6.37.7). Finally, we could pick the edge that goes from one end of the path of matchings to the fourth vertex in the path of matchings, giving $\mathrm{GRR}_{8}$ (Figure 6.38.1). We now add in one extra vertex. Because of Lemma 6.3.4, the edge that determines the triangle must touch all four existing matching edges. There is only one edge that does this, giving us $\mathrm{GRR}_{9}$ (Figure 6.38.2). 
Finally, we start with the parallel (Figure 6.15.4) and try to add in a green triangle while remaining on five vertices. As before, we identify an edge for our green triangle to avoid. This edge cannot be parallel to any existing matching edge, leaving three choices. First, we could pick the edge that joins the ends of the path, giving GRR $_{10}$ (Figure 6.38.3). Next, we could pick an edge from the parallel class to an end of the path, giving $\operatorname{GRR}_{11}$ (Figure 6.38.4). Finally, we could select an edge from the parallel class to the centre of the path, giving $\mathrm{GRR}_{12}$ (Figure 6.38.5). We now add in one extra vertex. Because of Lemma 6.3.4, the edge that determines the triangle must touch all four existing matching edges. There is only one edge that does this, giving us $\mathrm{GRR}_{13}$ (Figure 6.38.6).

We now go through each configuration in turn and check for the existence of $U_{2,4}$-restrictions containing all three of the extension elements. These checks are done by the lines () function from Appendix 6.A.4. The function calls, and an interpretation of the output are given below.

$\operatorname{GRR}_{1}$ lines(4, $\left.[[0,2,4],[0,5],[1,4]]\right)$ outputs that $[a, b, c$, 3 ] is a line containing the three extension elements. This is $\beth$, shown in Figure 6.43.2.

$\operatorname{GRR}_{2}$ lines $(5,[[0,3,6],[1,9],[2,8]])$ outputs that there is no line containing the three extension elements and a clique element.

$\mathrm{GRR}_{3}$ lines(4, [ [0, 2, 4], [1, 4], [1,4] ]) outputs that there is no line containing the three extension elements and a clique element.

$\mathrm{GRR}_{4}$ This is CF (Figure 6.12.5), and so is forbidden.

$\mathrm{GRR}_{5}$ lines $(5,[[7,8,9],[1,9],[5,8]])$ outputs that there is no line containing the three extension elements and a clique element.

$\mathrm{GRR}_{6}$ This is FS (Figure 6.13.2), and so is forbidden.

$\mathrm{GRR}_{7}$ This is HN (Figure 6.13.1), and so is forbidden.

$\mathrm{GRR}_{8}$ This is HM (Figure 6.13.4), and so is forbidden.

$\mathrm{GRR}_{9}$ This is BP (Figure 6.13.6), and so is forbidden.

$\operatorname{GRR}_{10}$ lines (5, [ [1, 2, 7], $\left.\left.[1,5],[1,9]\right]\right)$ outputs that there is no line containing the three extension elements and a clique element.

$\mathrm{GRR}_{11}$ lines (5, [ [7, 8, 9], [1, 5] , [1, 9] ]) outputs that there is no line containing the three extension elements and a clique element.

$\mathrm{GRR}_{12}$ This is WB (Figure 6.13.3), and so is forbidden.

$\operatorname{GRR}_{13}$ lines $(6,[[1,4,11],[0,13],[0,14]])$ outputs that there 


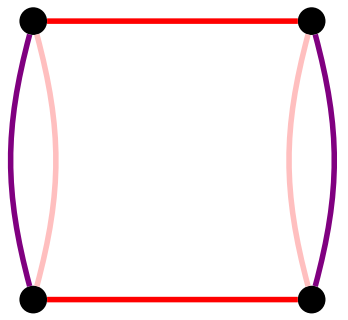

6.39.1: $\mathbf{R R R}_{1}$

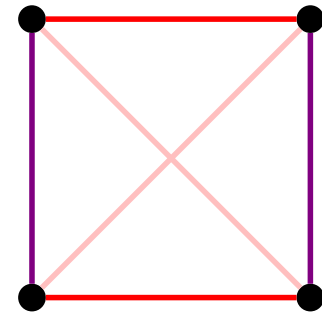

6.39.2: $\mathbf{R R R}$

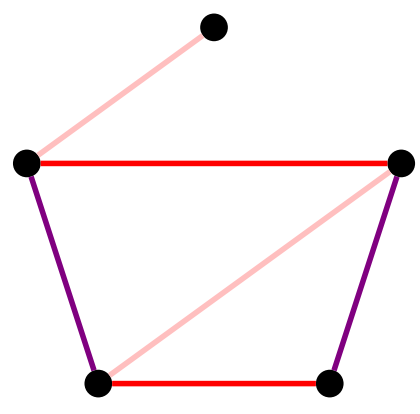

6.39.3: $\mathbf{R R R}_{3}$

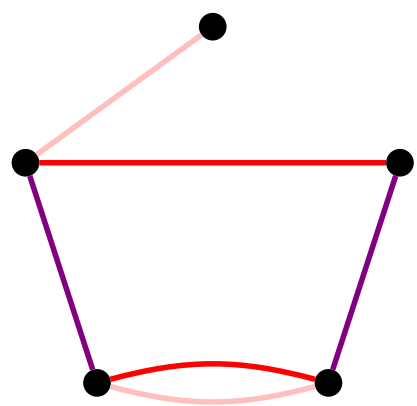

6.39.4: $\mathrm{RRR}_{4}$

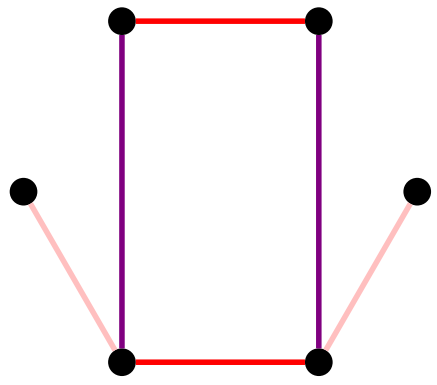

6.39.6: $\mathbf{R R R}_{6}$

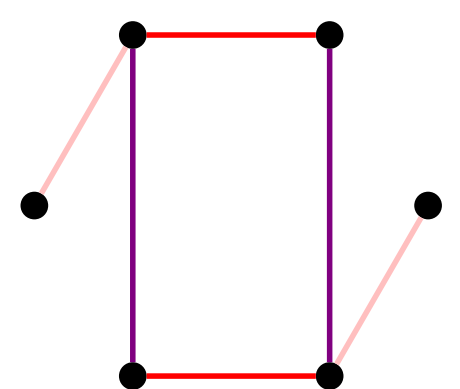

6.39.5: $\mathrm{RRR}_{5}$

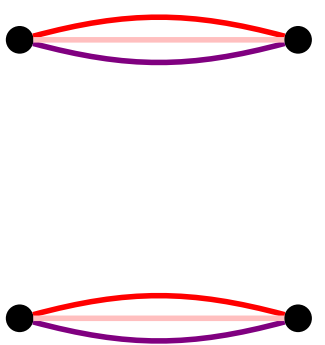

6.39.7: $\mathbf{R R R}_{7}$

Figure 6.39: Three Red Matchings I

is no line containing the three extension elements and a clique element.

So $\mathrm{GRR}_{1}$ is the only configuration of interest.

\subsubsection{Three Red Matchings}

From Section 6.4.1.3 we know that the first two red matchings must form a square, a double, a join, or a parallel. We systematically add in the third matching in all possible ways.

First note that we cannot add in more than two new vertices, as adding in three new vertices would force one red matching edge to be vertex-disjoint 

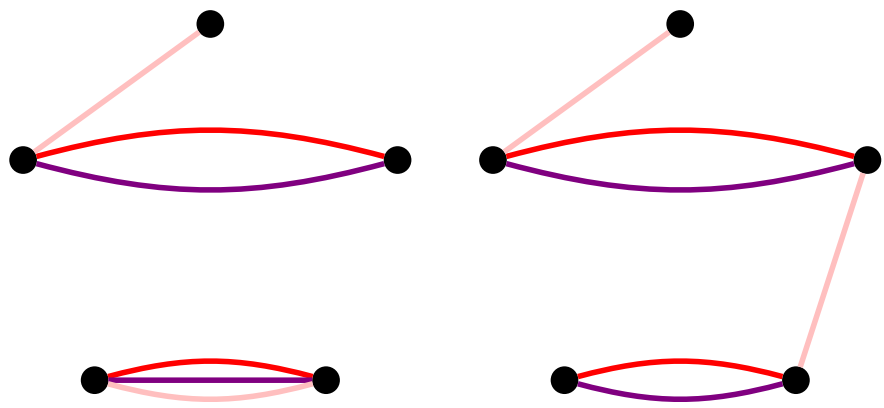

6.40.1: $\mathbf{R R R}_{8}$

6.40.2: RRR9

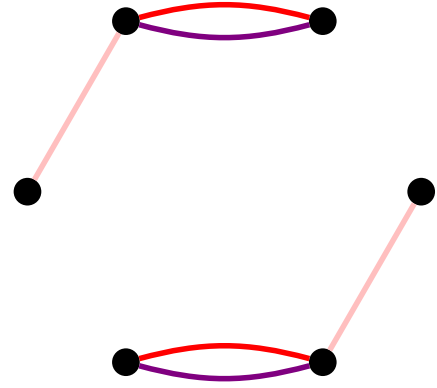

6.40.3: $\mathbf{R R R}_{10}$

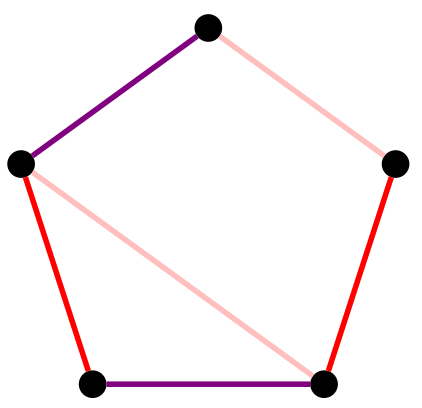

6.40.5: $\mathbf{R R R}_{12}$

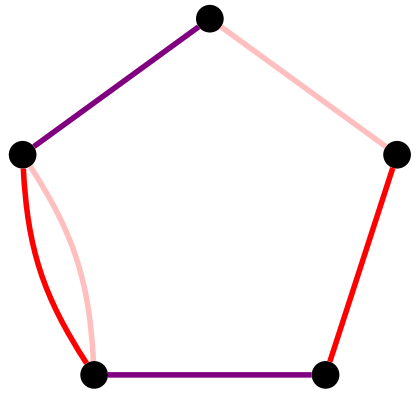

6.40.4: $\mathbf{R R R}_{11}$

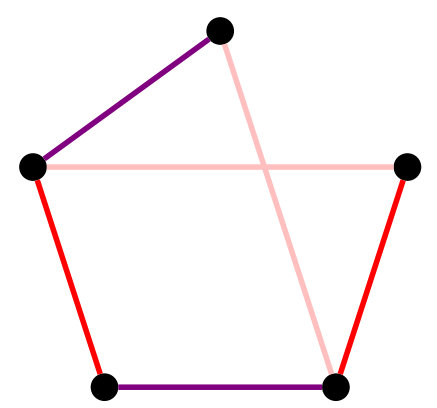

6.40.6: $\mathbf{R R R}_{13}$

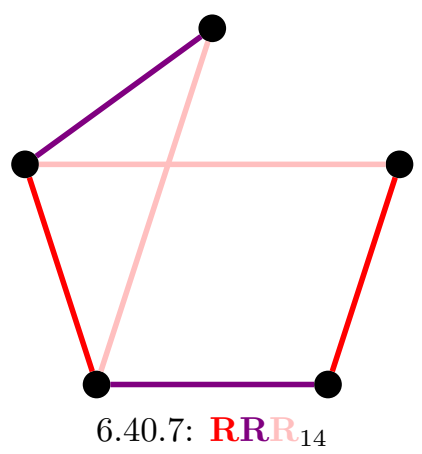

Figure 6.40: Three Red Matchings II 


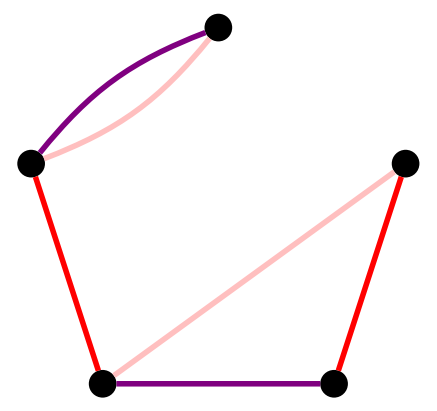

6.41.1: $\mathbf{R R R}_{15}$

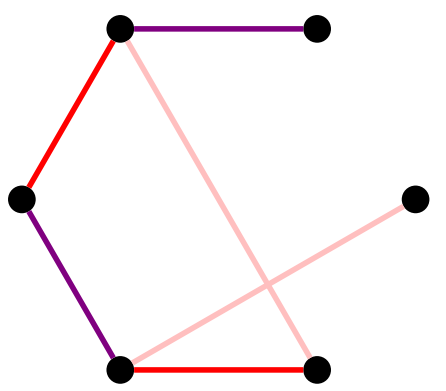

6.41.3: $\mathbf{R R R}_{17}$

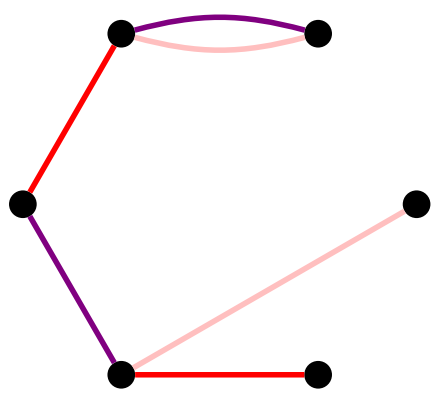

6.41.5: $\mathbf{R R R}_{19}$

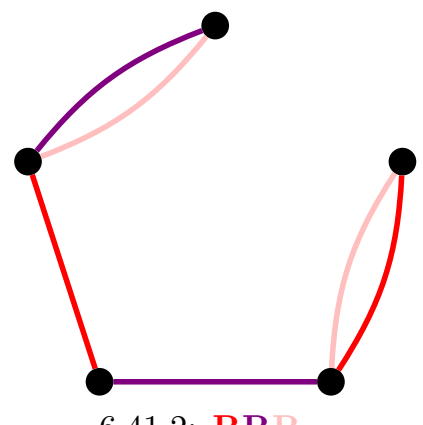

6.41.2: $\mathbf{R R R}_{16}$

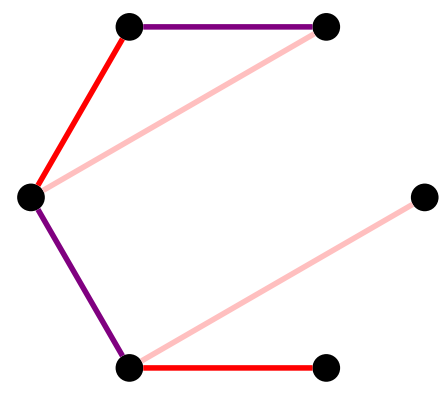

6.41.4: $\mathbf{R R R}_{18}$

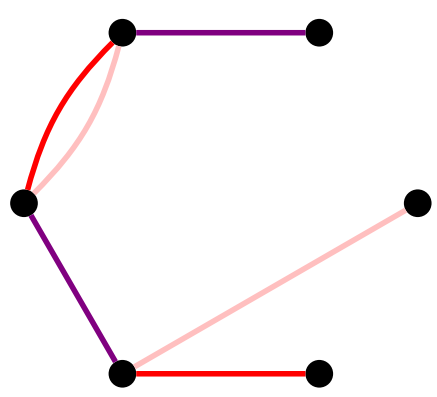

6.41.6: $\mathbf{R R R}_{20}$

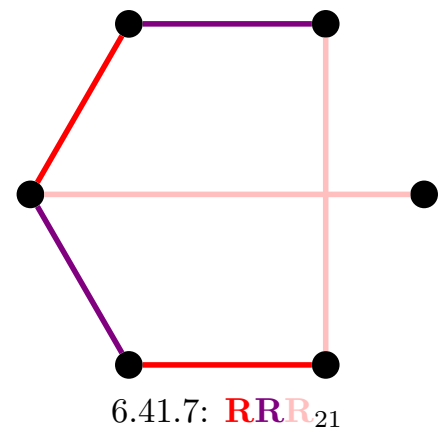

6.41.7: RRR 21

Figure 6.41: Three Red Matchings III 


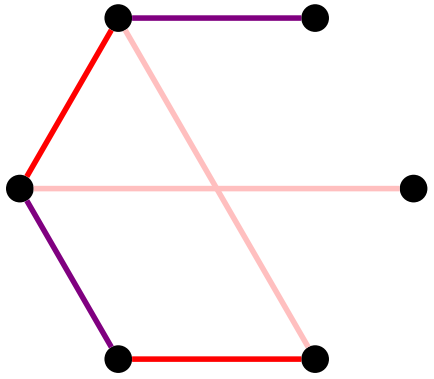

6.42.1: $\mathbf{R R R}_{22}$

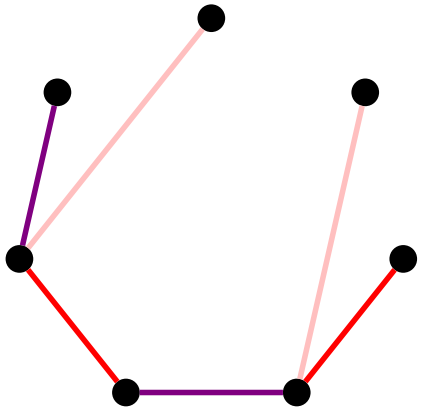

6.42.3: $\mathbf{R R R}_{24}$
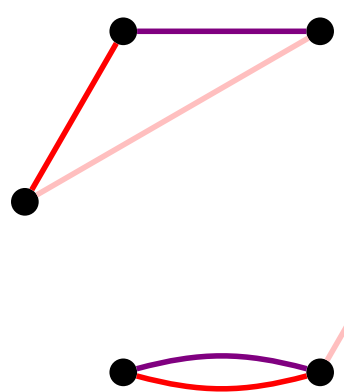

6.42.5: $\mathbf{R R} R_{26}$
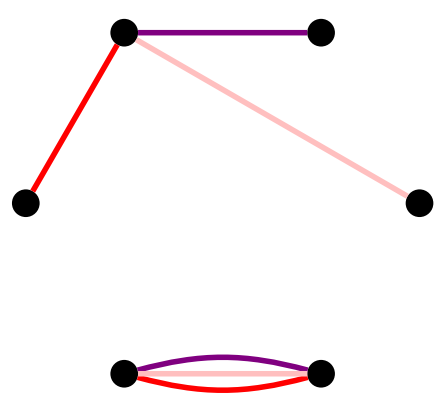

6.42.7: $\mathbf{R R R}_{28}$
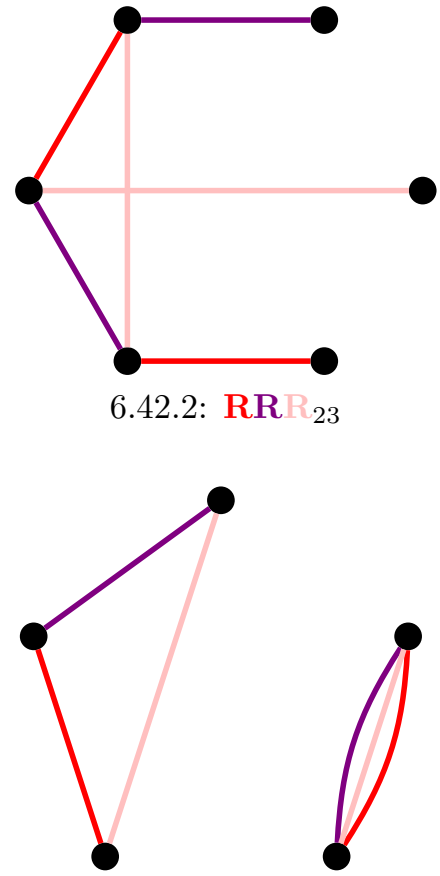

6.42.4: $\mathbf{R R R}_{25}$

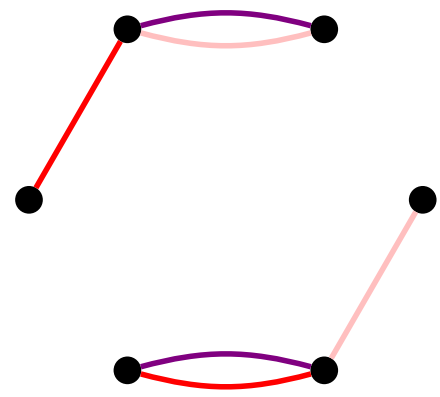

6.42.6: RRR 27

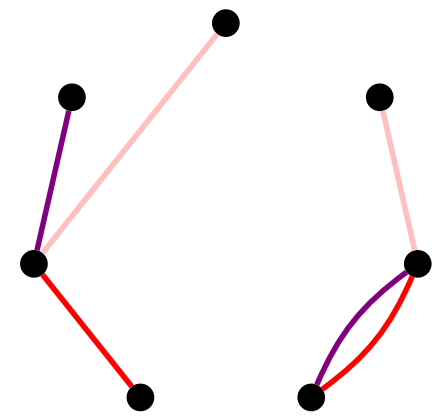

6.42.8: $\mathbf{R R R}_{29}$

Figure 6.42: Three Red Matchings IV 
from all the other matchings, creating a contradiction to Lemma 6.3.5.

Our first case is starting from the square (Figure 6.15.1) and remaining on four vertices. We can either add our third matching in parallel to one of the existing matchings, giving $\mathrm{RRR}_{1}$ (Figure 6.39.1), or we can add our third matching so that it is edge-disjoint from both existing matchings, giving $\mathrm{RRR}_{2}$ (Figure 6.39.2).

Our next case is again starting from the square, but this time we have five vertices. All the vertices in the square can be considered to be the same, so there is only one place that the first edge of the new matching can go up to symmetry. This leaves two places that the second edge can go. First, it could go diagonally across the square, giving $\mathrm{RRR}_{3}$ (Figure 6.39.3). The other place it could go is parallel to an existing matching edge, giving $\mathrm{RRR}_{4}$ (Figure 6.39.4).

We now start from a square again, but add in two extra vertices. The two vertices on the square that the last red matching edges are incident with can either be on a diagonal of the existing square $\left(\mathrm{RRR}_{5}\right.$, Figure 6.39.5), or be on an edge of the existing square $\left(\mathrm{RRR}_{6}\right.$, Figure 6.39.6). This finishes the case any two of the matchings form a square.

We now start from a double (Figure 6.15.2). Without increasing the number of vertices, we can either add our third matching in parallel to the existing matchings, giving $\mathrm{RRR}_{7}$ (Figure 6.39.7), or we could add the third matching so that it is edge-disjoint from the existing matching, giving a square $\left(R R R_{1}\right.$, Figure 6.39.1).

We now start from a double, but add in one extra vertex. The first edge of the third red matching can only go in one place, namely from the new vertex to an existing vertex. This leaves two possible places for the second edge to go. We can either add it in parallel to the avoided matching edge $\left(\mathrm{RRR}_{8}\right.$, Figure 6.40.1), or so that it is incident with both existing parallel classes $\left(\mathrm{RRR}_{9}\right.$, Figure 6.40.2).

This time, starting from the double, we add in two extra vertices. If the two edges of the third matching go to the same parallel class in the double, the avoided parallel class gives a contradiction to Lemma 6.3.5. So the edges must go to distinct parallel classes, giving $\mathrm{RRR}_{10}$ (Figure 6.40.3). This finishes the case of any two of the matchings being a double. 
We now start from a join (Figure 6.15.3), and stay on five vertices. To make describing the placement of the third red matching easier, we label the vertices along the path 1 through 5 .

First, we place the first edge of the third red matching between 1 and 5 . This leaves two places for the second edge to go, either between 3 and $4\left(R_{R R}\right.$, Figure 6.40.4), or between 2 and $4\left(\mathrm{RRR}_{12}\right.$, Figure 6.40.5).

Next, we place the first edge of the third red matching between 1 and 4 . This leaves three places for the second edge to go. We could place it between 2 and 3, giving a square $\left(\mathrm{RRR}_{4}\right.$, Figure 6.39 .4$)$, or we could place it between 2 and 5, giving $\mathrm{RRR}_{13}$ (Figure 6.40.6). Finally, we could place it between 3 and 5, giving $\mathrm{RRR}_{14}$ (Figure 6.40.7).

Next, we place the first edge of the third red matching between 1 and 3 . There are three locations to place the final edge of the third matching, two of which we have already seen. These are between 2 and $4\left(\mathrm{RRR}_{3}\right.$, Figure 6.39.3), and between 2 and $5\left(\mathrm{RRR}_{14}\right.$, Figure 6.40.7). Finally, we could place the final edge of the third red matching between 4 and 5, giving $\mathrm{RRR}_{15}$ (Figure 6.41.1). We now place the first edge of the final matching between 1 and 2 . This also gives three locations for the final edge, two of which we have already seen. These are between 3 and $4\left(\mathrm{RRR}_{9}\right.$, Figure 6.40.2), and between 3 and $5\left(\mathrm{RRR}_{15}\right.$, Figure 6.41.1). Finally, we could place it between 4 and 5 , giving $\mathrm{RRR}_{16}$ (Figure 6.41.2).

There are all possible places, up to symmetry, for the third red matching on five vertices, as now any placement would have to avoid both 1 and 5, leaving only three vertices.

We now start from a join, and add in one extra vertex. By symmetry, the edge of the third red matching that uses this vertex must go to either 2 or 3 (as otherwise we would have a contradiction to Lemma 6.3.5).

We start by joining it to 2 . This leaves six locations for the final edge of the third red matching to go. If we place it either between 1 and 5 or 1 and 3, we get a contradiction to Lemma 6.3.5. The remaining four locations for the final edge of the third red matching are between 1 and $4\left(R_{R R}\right.$, Figure 6.41.3), between 3 and $5\left(\mathrm{RRR}_{18}\right.$, Figure 6.41.4), between 4 and 5 $\left(\mathrm{RRR}_{19}\right.$, Figure 6.41.5), and between 3 and $4\left(\mathrm{RRR}_{20}\right.$, Figure 6.41.6). 
So now we place the first edge of the third red matching so that it meets the join at 3 . There are now four locations up to symmetry that we could place the final edge of the third matching. If we place it between 1 and 2 , we have a contradiction to Lemma 6.3.5. The remaining three locations are between 1 and $5\left(\mathrm{RRR}_{21}\right.$, Figure 6.41.7), between 1 and $4\left(\mathrm{RRR}_{22}\right.$, Figure 6.42.1), and between 2 and $4\left(\mathrm{RRR}_{23}\right.$, Figure 6.42.2).

Now we start from a join, and add in two extra vertices. As already discussed, the third matching edges must meet the join at 2,3 , or 4 . If they meet the join at 2 and 3, we have a contradiction to Lemma 6.3.5. By symmetry, they cannot meet the join at 3 and 4 . If they meet the join at 2 and 4 , we get $\mathrm{RRR}_{24}$ (Figure 6.42.3). This finishes the case of any two of the red matchings forming a join.

Finally, we start from a parallel (Figure 6.15.4), firstly having no extra vertices. If we place the first edge of the third matching in the existing parallel class, there are two locations for the second edge to go. First, we could place it parallel to one of the existing edges, giving $R_{R} R_{8}$ (Figure 6.40.1). Secondly, we could place it so that it joins the ends of the existing path, giving $R_{R} R_{25}$ (Figure 6.42.4). If we place the first edge so that it is not in the existing parallel class, we must place it so that it joins the parallel class to the path. If we connect to an end of the path, the second edge of the third red matching could go in three places. First, we could place it so that it is incident with the parallel class and the centre of the path of matchings, giving $R_{R R}$ (Figure 6.39.4). Second, we could place it so that it is incident with the parallel class and the available end of the path of matchings, giving $R_{R R}$ (Figure 6.40.4). Finally, we could place it parallel to an existing matching edge in the path of matchings, giving $\mathrm{RRR}_{16}$ (Figure 6.41.2). Finally, if we place the third red matching so that neither edge of the red matching joins the existing parallel component to an end of the path of matchings, we only have $\operatorname{RRR}_{15}$ (Figure 6.41.1).

We now start from a parallel and add in one extra vertex. The edge in the third matching that uses this extra vertex must go to either the parallel class or the centre of the path, as otherwise we would have a contradiction to Lemma 6.3.5. We start by joining to the parallel class. The final edge in the third matching can go in four different places. If we place it from the parallel class to an end of the path, we have a contradiction to Lemma 6.3.5. If we 
place it from the parallel class to the centre of the path, we get $\mathrm{RRR}_{20}$ (Figure 6.41.6). The other two locations for the final edge of the third matching are joining the ends of the path $\left(\mathrm{RRR}_{26}\right.$, Figure 6.42.5) and parallel to one of the path edges $\left(\mathrm{RRR}_{27}\right.$, Figure 6.42.6). If we now add the first edge of the third red matching so that it goes to the centre of the path, we have three places for the final edge to go. If we join the ends of the path, we have a contradiction to Lemma 6.3.5. If we join the parallel class to the matching, we get $\mathrm{RRR}_{19}$ (Figure 6.41.5). Finally, we could place the final edge in the existing parallel class, giving $\mathrm{RRR}_{28}$ (Figure 6.42.7).

For the last case, we start from a parallel and add in two extra vertices. As discussed earlier, each edge of the third matching must either go to the parallel class or the centre of the path. If both edges go to the parallel class, we get a contradiction to Lemma 6.3.5. If one edge goes to the parallel class and the other edge goes to the centre of the path, we get $\mathrm{RRR}_{29}$ (Figure 6.42.8). This finishes the entire case check for three red matchings.

We now go through each configuration in turn and check for the existence of $U_{2,4}$-restrictions containing all three of the extension elements. These checks are done by the lines() function from Appendix 6.A.4. The function calls, and an interpretation of the output are given below.

$\operatorname{RRR}_{1}$ lines $(4,[[0,5],[0,5],[1,4]])$ outputs that there is no line containing the three extension elements and a clique element.

$\mathrm{RRR}_{2}$ This is XS (Figure 6.10.1), and so is forbidden.

$\mathrm{RRR}_{3}$ This is XG (Figure 6.11.1), and so is forbidden.

$\mathrm{RRR}_{4}$ lines $(5,[[1,9],[2,8],[5,8]])$ outputs that there is no line containing the three extension elements and a clique element.

$\mathrm{RRR}_{5}$ This contains a TT (Figure 6.9.3), and so is forbidden.

$\mathrm{RRR}_{6}$ This contains a TT (Figure 6.9.3), and so is forbidden.

$\mathrm{RRR}_{7}$ This is TM (Figure 6.10.2), and so is forbidden.

$\mathrm{RRR}_{8}$ This is AF (Figure 6.11.3), and so is forbidden.

$\mathrm{RRR}_{9}$ This is MW (Figure 6.10.4), and so is forbidden.

$\mathrm{RRR}_{10}$ This contains a TT (Figure 6.9.3), and so is forbidden.

$\mathrm{RRR}_{11}$ This is DM (Figure 6.10.5), and so is forbidden.

$\operatorname{RRR}_{12}$ lines (5, [ [0, 7], [1, 9], [5, 8]]) outputs that there is no line containing the three extension elements and a clique element.

$\mathrm{RRR}_{13}$ This is SW (Figure 6.11.5), and so is forbidden. 
$\mathrm{RRR}_{14}$ lines (5, [ [1,9], [2, 6], [5, 8]]) outputs that there is no line containing the three extension elements and a clique element.

$\mathrm{RRR}_{15}$ This is PL (Figure 6.11.4), and so is forbidden.

$\mathrm{RRR}_{16}$ This is BC (Figure 6.10.3), and so is forbidden.

$\mathrm{RRR}_{17}$ This contains a TT (Figure 6.9.3), and so is forbidden.

$\mathrm{RRR}_{18}$ This contains a TT (Figure 6.9.3), and so is forbidden.

$\mathrm{RRR}_{19}$ This contains a TT (Figure 6.9.3), and so is forbidden.

$\mathrm{RRR}_{20}$ lines $(6,[[0,14],[5,14],[7,13]])$ outputs that there is no line containing the three extension elements and a clique element.

$\mathrm{RRR}_{21}$ This contains a TT (Figure 6.9.3), and so is forbidden.

$\mathrm{RRR}_{22}$ This contains a TT (Figure 6.9.3), and so is forbidden.

$\mathrm{RRR}_{23}$ This is DI (Figure 6.11.6), and so is forbidden.

$\mathrm{RRR}_{24}$ This contains a TT (Figure 6.9.3), and so is forbidden.

$\mathrm{RRR}_{25}$ This is AE (Figure 6.11.2), and so is forbidden.

$\mathrm{RRR}_{26}$ This contains a TT (Figure 6.9.3), and so is forbidden.

$\mathrm{RRR}_{27}$ This contains a TT (Figure 6.9.3), and so is forbidden.

$\mathrm{RRR}_{28}$ lines $(6,[[0,11],[0,13],[0,14]])$ outputs that there is no

line containing the three extension elements and a clique element.

$\mathrm{RRR}_{29}$ This contains a TT (Figure 6.9.3), and so is forbidden.

So there are no configurations of interest.

\subsubsection{Summary}

We have now considered all possible ways to construct a $U_{2,4}$-restriction where exactly three of the elements are non-clique elements. There are two ways that this can occur, $\mathrm{GGR}_{3}$ (Figure 6.36.3), and $\mathrm{GRR}_{1}$ (Figure 6.37.1).

To summarise, there are four configurations in an augmented clique which may quadrate to a $U_{2,4}$-restriction containing a clique element in the corresponding matroid. These are a green triangle, a doubled-up red matching, and the two configurations ( $\aleph$ and $\beth$ ) from Figure 6.43. 


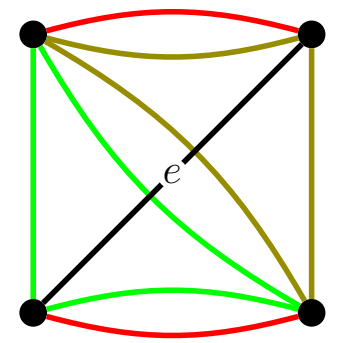

6.43.1:

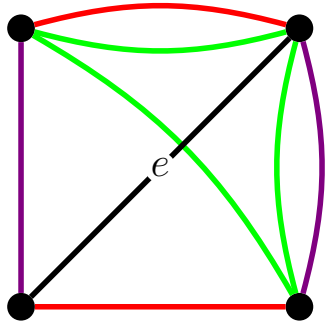

6.43.2: コ

Figure 6.43: $U_{2,4}$-restrictions

\subsection{Proof of Theorem 6.1.4}

In order to prove Theorem 6.1.4, we need to find an element of the spanning clique to contract. We want this element to only be on three-point lines. We start by restricting the location of $\aleph$ (Figure 6.43.1) and $\beth$ (Figure 6.43.2) configurations.

Lemma 6.6.1. Let $e$ be in a (Figure 6.43.1) configuration $\aleph_{e}$. It is not possible to have an edge $f$ that is vertex-disjoint from $\aleph_{e}$ and is in a $U_{2,4^{-}}$ restriction.

Proof. Assume for a contradiction that this does occur. So we have an edge $f$ such that $f$ is vertex-disjoint from $\aleph_{e}$ and $f$ is in a $U_{2,4}$-restriction.

There are four possible configurations that $f$ could be in, in order for $f$ to be in a $U_{2,4}$-restriction. First, it could either be on a green triangle or on a doubled-up red matching. It could also be in a $\aleph$ (Figure 6.43.1) or a $\beth$ (Figure 6.43.2).

If $f$ was on a green triangle, then one of the red matching edges in $\aleph_{e}$ would not touch this green triangle, contradicting Lemma 6.3.4.

If $f$ was on a red matching, then this red matching edge would be vertexdisjoint from a green triangle in $\aleph_{e}$, contradicting Lemma 6.3.4.

If $f$ was on either a $\aleph$ or a $\beth$, then we would need to place a red matching so both ends of $f$ and the two vertices in $\aleph_{e}$ that are not incident with $e$. However we place this red matching, we are able to find a forbidden TT (Figure 6.9.3) configuration with the red matching in $\aleph_{e}$.

As all possibilities have been exhausted, it is impossible for $f$ to be vertex- 
disjoint from $\aleph_{e}$ and in a $U_{2,4}$-restriction, as required.

Lemma 6.6.2. Let $e$ be in $a \beth$ (Figure 6.43.2) configuration $\beth_{e}$. It is not possible to have an edge $f$ that is vertex-disjoint from $\beth_{e}$ and is in a $U_{2,4^{-}}$ restriction.

Proof. Assume for a contradiction that this does not occur. So we have an edge $f$ such that $f$ is vertex-disjoint from $\beth_{e}$ and $f$ is in a $U_{2,4}$-restriction.

As in the proof of Lemma 6.6.1, there are four possible configurations that $f$ could be in, in order for $f$ to be in a $U_{2,4}$-restriction. First, it could either be on a green triangle or on a doubled-up red matching. It could also be in a $\aleph$ (Figure 6.43.1) or a $\beth$ (Figure 6.43.2).

If $f$ was on a green triangle, then one of the red matching edges in $\beth_{e}$ would not touch this green triangle, contradicting Lemma 6.3.4.

If $f$ was on a red matching, then this red matching edge would be vertexdisjoint from the green triangle in $\beth_{e}$, contradicting Lemma 6.3.4.

If $f$ was on either a $\aleph$ or a $\beth$, then we would need to place a red matching so that it touches two vertices of the green triangle in $\beth_{e}$ and both ends of $f$. Up to symmetry, there are two ways to do this, both of which contain a forbidden TT (Figure 6.9.3) configuration with a red matching in $\beth_{e}$.

As all possibilities have been exhausted, it is impossible for $f$ to be vertexdisjoint from $\beth_{e}$ and in a $U_{2,4}$-restriction, as required.

Lemma 6.6.3. Let $M$ be minor-minimal counterexample to Theorem 6.1.4 such that $M$ is maximum sized. There must be an element of a spanning clique of $M$ that is not in a $U_{2,4}$-restriction.

Proof. Assume for a contradiction that every clique element is in a $U_{2,4^{-}}$ restriction. Arbitrarily pick $e$ from the clique. From Lemmas 2.3.1 and 6.1.5 we know that the clique must have at least six vertices.

There are four possible configurations that $e$ could be in. First, it could either be on a green triangle or on a doubled-up red matching. It could also be in a $\aleph$ (Figure 6.43.1) or a $\beth$ (Figure 6.43.2).

Assume first that $e$ is on a $\aleph$ or a $\beth$. As we are on at least six vertices, we are able to find an edge $f$ that is vertex disjoint from the configuration containing e. It follows from Lemmas 6.6.1 and 6.6.2 that this is a contradiction. 
Hence $e$ cannot be in a $\aleph$ or a $\beth$ and we can assume that there are no $\aleph$ or $\beth_{\mathrm{s}}$ for the rest of this analysis.

Now we assume that $e$ is on a doubled-up red matching, and consider $f$ as before. As before, $f$ cannot be on a red matching, as this would contradict Lemma 6.3.5. Likewise, if $f$ was in a green triangle, then only one of the two existing parallel classes of red matchings would touch this new green triangle, contradicting Lemma 6.3.4. So e cannot be on a doubled-up red matching.

Now we assume that $e$ is in a green triangle $T_{e}$. Because the previous cases have considered everything else, every edge of our complete graph must be in a green triangle. Consider an edge $f$ that is vertex-disjoint from $T_{e}$, and consider a green triangle $T_{f}$ containing $f . T_{e}$ and $T_{f}$ must meet at a vertex $v$, as otherwise we would have a forbidden TL (Figure 6.8.3).

If we have seven or more vertices, then we can find an edge $g$ that is vertexdisjoint from both $T_{e}$ and $T_{f}$. Now we can find a green triangle containing $g$, and this green triangle must touch both $T_{e}$ and $T_{f}$, and so must contain $v$. Hence we have a forbidden RA (Figure 6.8.6), and so we have at most six vertices. It follows from Lemma 6.1.5 that we have at least six vertices, and hence we have exactly six vertices.

Let $u$ be the vertex that is not in $T_{e}$ or $T_{f}$. Let $T_{e}$ have vertices $v, v_{e}$, and $u_{e}$. Let $T_{f}$ have vertices $v, v_{f}$, and $u_{f}$. The edge $u v_{e}$ must be in a green triangle $T_{x}$. Because we cannot have vertex-disjoint green triangles by TL (Figure 6.8.3), up to symmetry, $T_{x}$ must contain either $v$ or $v_{f}$. If $T_{x}$ contains $v_{f}$, then we can find a forbidden TB (Figure 6.8.4), so $T_{x}$ must consist of the vertices $v u v_{e}$. Likewise, any green triangle $T_{y}$ containing the edge $u v_{f}$ must consist of the vertices $v u v_{f}$.

We now consider a green triangle $T_{z}$ containing the edge $u_{e} u_{f}$. This green triangle must contain either $u$ or $v$, as otherwise we would have vertex-disjoint green triangles, which are forbidden by TL (Figure 6.8.3). If $T_{z}$ contains $u$, then the green triangles $T_{f}, T_{y}$, and $T_{z}$ form a forbidden OM (Figure 6.8.2). Hence $T_{z}$ must consist of the vertices $u_{e} u_{f} v$, and the five green triangles $T_{e}$, $T_{f}, T_{x}, T_{y}$, and $T_{z}$ form a forbidden VQ (Figure 6.8.5).

As we covered every case, it is not possible for every clique element to be in a $U_{2,4}$-restriction, and so there must be an element of the clique that is not in a $U_{2,4}$-restriction, as desired. 
We now have two cases to consider in order to prove Theorem 6.1.4. The first case is when a clique element that is not on a $U_{2,4}$-restriction is on three non-clique three-point lines.

If a clique element $e$ is not in a $U_{2,4}$-restriction and is on three non-clique three-point lines, then it must be in one of the four configurations from Figure 6.34. These configurations are the line-star configuration, the twoparallel configuration, the matching-star configuration, and the Betsy Ross configuration.

We now analyse the four configurations.

Definition 6.6.4. Let $C=(G, \mathcal{A})$ be a golden-mean augmented clique. A semi-line-star configuration is a restriction $C^{\prime}=\left(G^{\prime}, \mathcal{A}^{\prime}\right)$ of $C$, where

(i) $G^{\prime}$ is a complete subgraph of $G$,

(ii) $G^{\prime}$ is spanned by a subgraph consisting of $H$ and $e=u v$, where

1. $H$ is a star, and

2. $e$ is vertex-disjoint from $H$.

In addition, $\mathcal{A}^{\prime}$ consists of a collection of green triangles, where

(i) each green triangle is incident with either $u$ or $v$,

(ii) each green triangle contains an edge of $H$,

(iii) every edge of $H$ is in one or two green triangles, and

(iv) no two members of $\mathcal{A}^{\prime}$ have the same edge set.

A semi-line-star configuration is maximal if it is not a restriction of a semiline-star configuration (apart from itself).

A line-star configuration is a semi-line-star configuration where every edge in $H$ is in two green triangles in $\mathcal{A}^{\prime}$.

A schematic is given in Figure 6.44.

Lemma 6.6.5. Let $C=(G, \mathcal{A})$ be a golden-mean augmented clique. Let $C_{1}=\left(G_{1}, \mathcal{A}_{1}\right)$ be a maximal semi-line-star configuration within $C$, and assume that $C_{1}$ has a restriction $C_{2}=\left(G_{2}, \mathcal{A}_{2}\right)$, such that $C_{2}$ is a line-star configuration containing at least six vertices. Then there are no green triangles in $\mathcal{A} \backslash \mathcal{A}_{1}$.

Proof. We use the labels from Figure 6.44, where $u, v, s, l_{1}, l_{2}$, and $l_{3}$ are in $G_{2}$. Let $T$ be a green triangle in $\mathcal{A} \backslash \mathcal{A}_{1}$. If $T$ does not touch $s$, then we 


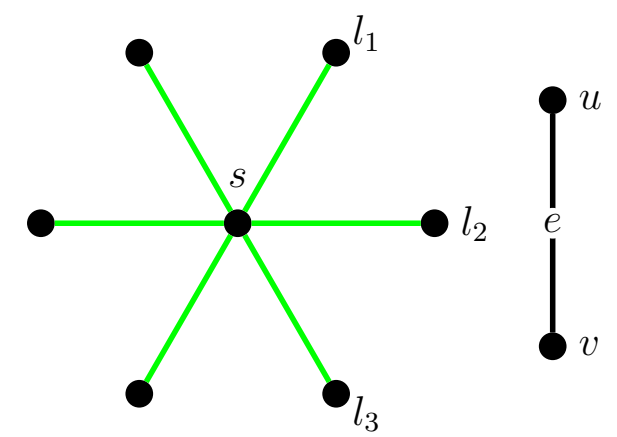

Figure 6.44: Line-star configuration

are able to find a TL (Figure 6.8.3), which is forbidden, so $T$ must touch $s$. Choose two leaves $\left(l_{1}\right.$ and $\left.l_{2}\right)$ in the star, with $T_{1}=l_{1} s u$ and $T_{2}=l_{2} s v$ as green triangles. $T_{1}$ and $T_{2}$ are edge-disjoint, so $T$ must share an edge with one of $T_{1}$ or $T_{2}$, as if it does not, we will be able to find a RA (Figure 6.8.6), which is forbidden. Without loss of generality, $T$ shares an edge with $T_{1}$, and so $T$ contains either $u$ or $l_{1}$.

We consider $u$ first. So $T=$ sux. If $x=v$, then $e$ is on a green triangle and hence is in a $U_{2,4}$-restriction, which is forbidden by assumption. If $x=l_{i}$ for $i \in\{1,2,3\}$, then we have a $\Delta$ (Figure 6.24.2) configuration, with one of the green triangles doubled up. In this case, is_forbidden $(5,[0,1,4]$, $[0,3,6],[1,2,7],[2,3,9],[2,3,9]])$ reports that this configuration is forbidden. So $x$ cannot be $s, l_{i}, u$, or $v$. If $x$ is not in $G_{1}$, then we contradict the maximality of $C_{1}$. So $x$ is in $G_{1}$. The maximality of $C_{1}$ means that $T$ is identical to, but distinct from, a green triangle that is already in $\mathcal{A}^{\prime}$. In this case, we can find a forbidden configuration consisting of a maximal semi-line-star configuration with star $S$ on six vertices with five green triangles, with an edge $f$ of $S$ that is only on one green triangle. The green triangle containing $f$ is doubled-up, giving us the function call is_forbidden $(6,[[1,3,10],[1,3,10],[5,7,10],[5$, $8,11],[9,10,12],[9,11,13]])$, which reports that this configuration is forbidden.

Hence $T$ cannot touch $u$, and so $T=s l_{1} x$. By symmetry, $x \neq$ $u$ and $x \neq v$. If $x$ is not in $G_{2}$, then the three green triangles $T, s l_{2} u$, and $s l_{3} v$ form a RA (Figure 6.8.6), which is forbidden. Hence, without loss of generality, $x=l_{2}$. So we have the 


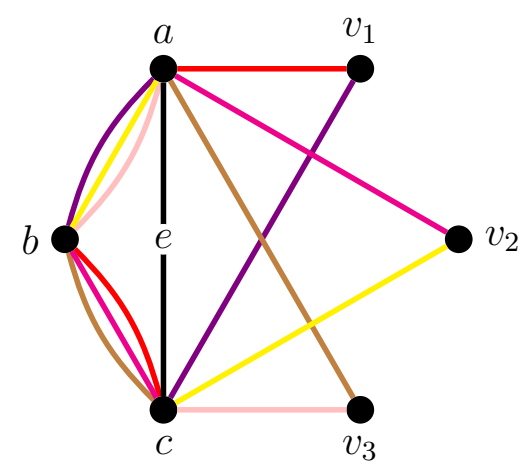

Figure 6.45: Two-parallel configuration

configuration shown in Figure 6.26.2 with an additional green triangle $\{2,4,13\}$. The function call is_forbidden $(6,[[0,1,5],[0,2,6]$, $[0,4,8],[1,3,10],[2,3,12],[2,4,13],[3,4,14]])$ outputs True, which means that this configuration is forbidden.

As all possibilities have been exhausted, there can be no green triangles in $\mathcal{A} \backslash \mathcal{A}_{1}$.

Lemma 6.6.6. Let $C=(G, \mathcal{A})$ be a golden-mean augmented clique. Let $C_{1}=\left(G_{1}, \mathcal{A}_{1}\right)$ be a maximal semi-line-star configuration within $C$, and assume that $C_{1}$ has a restriction $C_{2}=\left(G_{2}, \mathcal{A}_{2}\right)$, such that $C_{2}$ is a line-star configuration containing at least six vertices. Then there are no red matchings in $\mathcal{A} \backslash \mathcal{A}_{1}$.

Proof. Let $R$ be a red matching in $\mathcal{A} \backslash \mathcal{A}_{1}$. Because of Lemma 6.3.4, both edges of $R$ must touch all the green triangles in $\mathcal{A}_{1}$. This means that both edges of $R$ must either touch $s$ or be $e$.

Assume that one edge of $R$ is $e$. No matter where the other edge of $R$ is, as it must touch $s$, we are able to find a forbidden KF (Figure 6.12.2).

Hence both edges of $R$ must touch $s$, which is impossible.

Definition 6.6.7. Let $C=(G, \mathcal{A})$ be a golden-mean augmented clique. A semi-two-parallel configuration is a restriction $C^{\prime}=\left(G^{\prime}, \mathcal{A}^{\prime}\right)$ of $C$, where

(i) $G^{\prime}$ is a complete subgraph of $G$, and

(ii) $G^{\prime}$ is spanned by the vertices $\left\{a, b, c, v_{1}, \ldots, v_{n}\right\}$. 
Let $e$ be the edge $a c$. In addition, $\mathcal{A}^{\prime}$ consists of a collection of red matchings, where

(i) each red matching contains either $a b$ or $b c$,

(ii) if a red matching contains $a b$, then it also contains $c v_{i}$, for some $i \in$ $1, \ldots, n$,

(iii) if a red matching contains $b c$, then it also contains $a v_{i}$, for some $i \in$ $1, \ldots, n$,

(iv) All red matchings in $\mathcal{A}^{\prime}$ are distinct, and

$(\mathrm{v})$ each $v_{i}$ is in either one or two red matchings.

A semi-two-parallel configuration is maximal if it is not a restriction of a semi-two-parallel configuration (apart from itself).

A two-parallel configuration is a semi-two-parallel configuration where every $v_{i}$ in $G^{\prime}$ is contained in two red matchings in $\mathcal{A}^{\prime}$.

A schematic is given in Figure 6.45.

Lemma 6.6.8. Let $C=(G, \mathcal{A})$ be a golden-mean augmented clique. Let $C_{1}=\left(G_{1}, \mathcal{A}_{1}\right)$ be a maximal semi-two-parallel configuration within $C$, and assume that $C_{1}$ has a restriction $C_{2}=\left(G_{2}, \mathcal{A}_{2}\right)$ such that $C_{2}$ is a two-parallel configuration containing at least six vertices. Then there are no green triangles in $\mathcal{A} \backslash \mathcal{A}_{1}$.

Proof. We use the labels from Figure 6.45, where $a, b, c, v_{1}, v_{2}$, and $v_{3}$ are in $G_{2}$. Let $T$ be a green triangle in $\mathcal{A} \backslash \mathcal{A}_{1}$.

First, assume that $T$ consists of three vertices from $G \backslash G_{1}$. But this is a contradiction to Lemma 6.3.4.

There is no vertex in $G_{1}$ that every red matching edge from $\mathcal{A}_{2}$ touches, so it is impossible for $T$ to contain two vertices from $G \backslash G_{1}$ without contradicting Lemma 6.3.4.

The only edge of $G_{1}$ that all red matching edges touch is $a c$. However, this is also $e$, and hence cannot be on a green triangle as that would cause $e$ to be in a $U_{2,4}$-restriction, which is forbidden by assumption. Therefore there is no way that $T$ can contain one vertex from $G \backslash G_{1}$ without contradicting Lemma 6.3.4.

Therefore $T$ must be on vertices from $G_{1}$. Every $K_{3}$ subgraph of $G_{1}$ that all 
red matching edges from $\mathcal{A}_{1}$ touch contains $e$, which is a contradiction to the definition of $e$.

Hence there are no green triangles in $\mathcal{A} \backslash \mathcal{A}_{1}$.

Lemma 6.6.9. Let $C=(G, \mathcal{A})$ be a golden-mean augmented clique. Let $C_{1}=\left(G_{1}, \mathcal{A}_{1}\right)$ be a maximal semi-two-parallel configuration within $C$, and assume that $C_{1}$ has a restriction $C_{2}=\left(G_{2}, \mathcal{A}_{2}\right)$ such that $C_{2}$ is a two-parallel configuration containing at least six vertices. Then there are no red matchings in $\mathcal{A} \backslash \mathcal{A}_{1}$.

Proof. We use the labels from Figure 6.45, where $a, b, c, v_{1}, v_{2}$, and $v_{3}$ are in $G_{2}$. Let $R$ be a red matching in $\mathcal{A} \backslash \mathcal{A}_{1}$.

First note that Lemma 6.3.5 implies that $R$ cannot use three or more vertices from $G \backslash G_{1}$.

Assume that $R$ contains two vertices, $u$ and $v$, from $G \backslash G_{1}$. Because of Lemma 6.3.5, the edges of $R$ must touch all red matching edges from the red matchings in $\mathcal{A}_{1}$. Hence, up to symmetry, $R=(u a, v c)$ and we can find a forbidden TT (Figure 6.9.3) using $R$ and red matching from $\mathcal{A}_{1}$.

Now assume that $R=\left(r_{1}, r_{2}\right)$ contains one vertex $v$ from $G \backslash G_{1}$. The vertices in $G_{1}$ that touch every red matching in $\mathcal{A}_{1}$ are $a, b$, and $c$. Hence $r_{2}$, the edge of $R$ that uses $v$, must meet $G_{1}$ at one of $a, b$, or $c$. We now consider the possible locations for $r_{1}$. If $r_{1}$ is only incident with vertices in $\left\{v_{1}, \ldots, v_{n}\right\}$, then it can touch only four different red matchings, which is not enough to satisfy Lemma 6.3 .5 as $\mathcal{A}_{2}$ consists of at least six red matchings. Hence $r_{1}$ must be incident with at least one of $a, b$, or $c$. We consider the cases in turn.

First, we consider the case where $r_{2}$ meets $G_{1}$ at $a$. Note that this case is the same as when $r_{2}$ meets $G_{1}$ at $c$. First, it could be that $r_{1}=b c$. This contradicts the maximality of $C_{1}$. Next, consider the case where $r_{1}=b v_{i}$. In this case, we are able to find a forbidden TT (Figure 6.9.3) that uses $R$ and a red matching from $\mathcal{A}_{1}$ that avoids $v_{i}$ and does not contain $a b$. Finally, we consider the case where $r_{2}=c v_{i}$. In this case, we are able to find a forbidden TT (Figure 6.9.3) consisting of $R$ and a red matching from $\mathcal{A}_{1}$ that contains bc and avoids $v_{i}$.

Therefore $r_{2}$ meets $G_{1}$ at $b$. First, we consider the case that $r_{1}=a c$. This 
gives us a forbidden XG (Figure 6.11.1) consisting of $R$ and any two red matchings from $\mathcal{A}_{1}$ that form a RR-square (Figure 6.17.3). Hence, up to symmetry, $r_{1}=a v_{i}$. In this case, we are able to find a forbidden TT (Figure 6.9.3) that consists of $R$ and a red matching from $\mathcal{A}_{1}$ of the form $\left(a v_{j}, b c\right)$, where $i \neq j$.

So $R=\left(r_{1}, r_{2}\right)$ contains only vertices from $G_{1}$. By Lemma 6.3.5, both $r_{1}$ and $r_{2}$ must touch every red matching in $\mathcal{A}_{1}$. Hence both $r_{1}$ and $r_{2}$ must touch at least one of $a, b$, or $c$. We start by considering the case where $r_{1}$ touches two of $a, b$, and $c$.

If $r_{1}=a c$, then, by Lemma 6.3.5, $r_{2}=b v_{i}$ for some $i \in 1, \ldots, n$. In this case, we can find a forbidden XG (Figure 6.11.1). Now assume that $r_{1}=a b$. By Lemma 6.3.5, $r_{2}$ must touch $c$. So, by the maximality of $C_{1}, R$ must be parallel to an existing red matching. We can easily find a forbidden MW (Figure 6.10.4) in this case. This case is identical to the case where $r_{1}=b c$.

Therefore both $r_{1}$ and $r_{2}$ must touch exactly one vertex from $\{a, b, c\}$. First, we consider the case where $r_{1}=a x$ and $r_{2}=c y$, where $\{x, y\} \subset\left\{v_{1}, \ldots, v_{n}\right\}$. No matter what $x$ and $y$ are, we are always able to find a forbidden TT (Figure 6.9.3) consisting of $R$ and a red matching from $\mathcal{A}_{1}$ that avoids both $x$ and $y$. Hence, without loss of generality, $r_{1}=a x$ and $r_{2}=b y$, where $\{x, y\} \subset\left\{v_{1}, \ldots, v_{n}\right\}$. In this case, there exists a matching edge $c z$, where $z \in\left\{v_{1}, \ldots, v_{n}\right\} \backslash\{x, y\}$ that avoids $R$, a contradiction to Lemma 6.3.5.

Hence there are no red matchings in $\mathcal{A} \backslash \mathcal{A}_{1}$.

Definition 6.6.10. Let $C=(G, \mathcal{A})$ be a golden-mean augmented clique. A matching-star configuration is a restriction $C^{\prime}=\left(G^{\prime}, \mathcal{A}^{\prime}\right)$ of $C$, where

(i) $G^{\prime}$ is a complete subgraph of $G$,

(ii) $G^{\prime}$ is spanned by a subgraph consisting of $H$ and $e$, where

1. $H$ is a star with core $s$, and

2. $e$ is an edge that is vertex-disjoint from $H$.

In addition, $\mathcal{A}^{\prime}$ consists of a collection of red matchings, where

(i) each red matching contains $e$,

(ii) each red matching contains an edge of $H$, and

(iii) every edge of $H$ is in exactly one red matching.

A matching-star configuration is maximal if it is not a restriction of a 


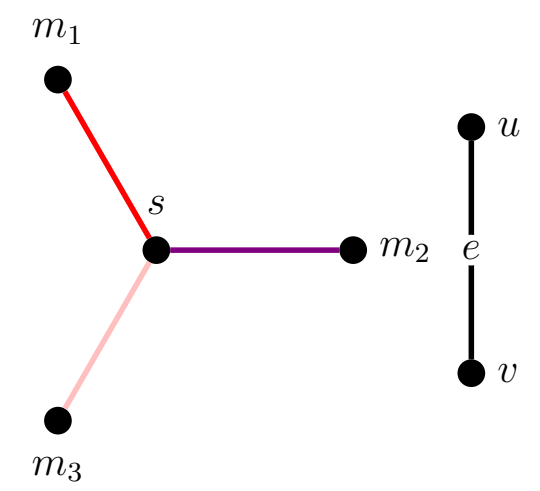

Figure 6.46: Matching-star configuration

matching-star configuration (apart from itself).

A schematic is given in Figure 6.46.

Lemma 6.6.11. Let $C=(G, \mathcal{A})$ be a golden-mean augmented clique. Let $C^{\prime}=\left(G^{\prime}, \mathcal{A}^{\prime}\right)$ be a maximal matching-star configuration within $C$. Let $e, s$ and $H$ be from the definition of $G^{\prime}$, and let $X=G \backslash\{s, u, v\}$. Then the endvertices of e can be labelled $u$ and $v$ in such a way that one of the following holds.

(i) (a) $\mathcal{A} \backslash \mathcal{A}^{\prime}$ contains no red matchings,

(b) if $T$ is a green triangle from $\mathcal{A}$, then $T=$ sux for some $x \in X$, and

(c) no two green triangles in $\mathcal{A}$ have the same edge set.

(ii) (a) $\mathcal{A}$ contains no green triangles,

(b) if $R$ is a red matching from $\mathcal{A} \backslash \mathcal{A}^{\prime}$, then $R=(s u, v x)$ for some $x \in X$, and

(c) no two red matchings in $\mathcal{A} \backslash \mathcal{A}^{\prime}$ have the same edge set.

Proof. Throughout this proof, we use the labels from Figure 6.46. Note that $C^{\prime}$ must have at least six vertices.

If $\mathcal{A}=\mathcal{A}^{\prime}$, then the lemma vacuously holds. Hence we can assume that $\mathcal{A} \neq \mathcal{A}^{\prime}$.

First assume that $\mathcal{A}$ contains a green triangle $T$. We need to show that $T=\operatorname{sux}$ for some $x \in X$.

Sublemma 6.6.11.1. Up to swapping the labels on $u$ and $v, T=$ sux for some $x \in X$. 
Subproof. If $T$ does not touch at least one of $u$ or $v$, then we have a contradiction to Lemma 6.3.4. The same thing occurs if $T$ does not touch $s$. Note that $T$ cannot touch both $u$ and $v$, as we are assuming that $e$ is not in a $U_{2,4}$-restriction. Hence, without loss of generality, $T=$ suz for some $z \in G \backslash\{s, u, v\}$, and we have the desired result.

Sublemma 6.6.11.1 is not enough, however. We need every green triangle in $\mathcal{A}$ to be of the form sux.

Sublemma 6.6.11.2. Assume that there are two distinct green triangles, $T$ and $T^{\prime}$ in $\mathcal{A}$. Then up to swapping the labels on $u$ and $v, T=$ sux and $T^{\prime}=$ suy, where $\{x, y\} \subseteq X$.

Subproof. From Sublemma 6.6.11.1, we can assume that $T=$ sux for some $x \in X$. Also, $T^{\prime}=$ swy, where $w \in\{u, v\}$ and $y \in X$. We first show that $y \neq x$.

Assume that $y=x$, that is, $T^{\prime}=s w x$. If $w=u$, then the two green triangles $T$ and $T^{\prime}$, along with the red matching $(u v, s z)$, for some $z \in X \backslash\{x\}$ form a forbidden CC (Figure 6.12.4). So $T^{\prime}=s v x$. In this case, the two green triangles $T$ and $T^{\prime}$, and the red matching $(u v, s z)$, for some $z \in X \backslash\{x\}$ form a KF (Figure 6.12.2), which is forbidden. Therefore $y \neq x$.

Therefore if the sublemma is false, $T^{\prime}=$ svy. In this case, the two green triangles $T$ and $T^{\prime}$, and the red matching (uv,sz), for some $z \in X \backslash\{x, y\}$, from a forbidden SR (Figure 6.13.5), and hence the sublemma is true.

From Sublemmas 6.6.11.1 and 6.6.11.2, we deduce that, if there are no red matchings in $\mathcal{A} \backslash \mathcal{A}^{\prime}$, then (i) holds.

We set aside the assumption that $\mathcal{A} \backslash \mathcal{A}^{\prime}$ contains a green triangle for now, and assume that there is one red matching $R$ in $\mathcal{A} \backslash \mathcal{A}^{\prime}$. We need to show that $R=(s u, v x)$ for some $x \in X$.

Sublemma 6.6.11.3. Up to swapping the labels on $u$ and $v, R=(s u, v x)$ for some $x \in X$.

Subproof. Let $R=\left(r_{1}, r_{2}\right)$, and let $\{i, j\}=\{1,2\}$. As every red matching edge must touch every other red matching by Lemma 6.3.5, each edge of $R$ must touch at least one of $s, u$, or $v$. 
First, assume that $R$ does not touch $s$. If $r_{i}=u v$, then $r_{j}$ cannot touch $s$, $u$, or $v$, which is a contradiction. Hence, without loss of generality, $r_{1}=u x$ and $r_{2}=v y$ for some $\{x, y\} \subseteq X$. However, in this case, the red matching edge $s z$, for some $z \in X \backslash\{x, y\}$, does not touch $R$, which is a contradiction to Lemma 6.3.5.

Therefore $R$ must touch $s$. Now assume that $R$ does not touch $v$ (note that this is equivalent to $R$ not touching $u$ ). Ergo, without loss of generality, $r_{1}=u x$ and $r_{2}=s y$ for some $\{x, y\} \subseteq X$. If either $x$ or $y$ is not in $G^{\prime}$, then we can easily find a forbidden TT (Figure 6.9.3). Hence we can assume that $\{x, y\} \subseteq H \backslash\{s\}$. In this case, the three red matchings $R,(u v, s x)$, and $(u v, s y)$ form a forbidden BC (Figure 6.10.3).

So $R$ must touch $u, v$, and $s$, meaning that the vertex set of $R$ is $\{u, v, s, x\}$ for some $x \in X$. There are three possible red matchings that $R$ could be $(u v, s x),(u x, s v)$, or $(s u, v x)$.

First, assume that $R=(u v, s x)$. If $x \notin G^{\prime}$, then we contradict the maximality of $C^{\prime}$, so we can assume that $x \in G$. In this case $R$ and two red matchings from $\mathcal{A}^{\prime},(u v, s x)$ and $(u v, s y)$, for some $y \in H \backslash\{s, x\}$ form a forbidden $\mathrm{AF}$ (Figure 6.11.3).

We now assume that $R=(u x, s v)$. We can swap the labels of $u$ and $v$ to get that $R=(s u, v x)$.

Hence $R=(s u, v x)$, as desired.

As before, Sublemma 6.6.11.3 is not enough. We need all red matchings in $\mathcal{A} \backslash \mathcal{A}^{\prime}$ to be of the form $(s u, v x)$.

Sublemma 6.6.11.4. Assume that there are two distinct red matchings, $R$ and $R^{\prime}$ in $\mathcal{A} \backslash \mathcal{A}^{\prime}$. Then, up to swapping the labels of $u$ and $v, R=(s u, v x)$ and $R^{\prime}=(s u, v y)$, where $\{x, y\} \subseteq X$.

Subproof. From Sublemma 6.6.11.3, we can assume that $R=(s u, v x)$ for some $x \in X$. Also, $R^{\prime}=(s t, w y)$, where $\{t, w\}=\{u, v\}$ and $y \in X$. We first show that $y \neq x$.

Assume that $y=x$, that is, $R^{\prime}=(s t, w x)$. If $t=u$, then the three red matchings $R, R^{\prime}$, and (uv,sz), for some $z \in X \backslash\{x\}$ form a MW (Figure 6.10.4), which is forbidden. Therefore $R^{\prime}=(s v, u x)$. In this case, the three red 
matchings $R, R^{\prime}$, and $(u v, s x)$ form a forbidden XG (Figure 6.11.1). So $y \neq x$.

Hence, if the sublemma is false, then $R^{\prime}=(s v, u y)$. In this case, the three red matchings $R, R^{\prime}$, and (uv,sz), for some $z \in X \backslash\{x, y\}$ form a forbidden DI (Figure 6.11.6), and hence the sublemma is true.

From Sublemmas 6.6.11.3 and 6.6.11.4, we can surmise that if there are no green triangles in $\mathcal{A} \backslash \mathcal{A}^{\prime}$, then (ii) holds.

We now have one last thing to show.

Sublemma 6.6.11.5. There cannot be a red matching $R$ and a green triangle $T$ in $\mathcal{A} \backslash \mathcal{A}^{\prime}$.

Subproof. Assume that both $R$ and $T$ are in $\mathcal{A} \backslash \mathcal{A}^{\prime}$. Without loss of generality, we can assume that $R=(s u, v x)$ for some $x \in X$. Then by Sublemma 6.6.11.1, $T=$ swy, where $w \in\{u, v\}$ and $y \in X$. We first show that $x \neq y$.

Assume that $x=y$, that is, $T=s w x$. If $w=u$, then $R, T$, and the red matching (uv,sz), for some $z \in X \backslash\{x\}$ form a forbidden HM (Figure 6.13.4). Hence $T=s v x$. In this case, $R, T$, and the red matching ( $u v, s z)$, for some $z \in X \backslash\{x\}$ form a HN (Figure 6.13.1), which is forbidden. Therefore $x \neq y$. As $C^{\prime}$ is maximal, $y \in X$. So $T=$ swy where $\{x, y\} \subseteq X$. If $w=u$, then the red matching edge $v x$ from $R$ does not touch $T$, a contradiction to Lemma 6.3.4. Ergo $T=$ svy. In this case, $R, T$, and the red matching (uv,sz) for some $z \in X \backslash\{x, y\}$ form a forbidden BP (Figure 6.13.6).

Therefore, if $R$ exist, then $T$ cannot exist, and the result follows.

We now have the desired result, as no other possibilities exist.

Definition 6.6.12. Let $C=(G, \mathcal{A})$ be a golden-mean augmented clique. A Betsy Ross configuration is a restriction $C^{\prime}=\left(G^{\prime}, \mathcal{A}^{\prime}\right)$ of $C$, where

(i) $G^{\prime} \cong K_{4}$,

(ii) $G^{\prime}$ consists of the vertices $a, b, c$, and $d$, and

(iii) $\mathcal{A}^{\prime}$ consists of two green triangles and three red matchings, where, up to relabelling, 


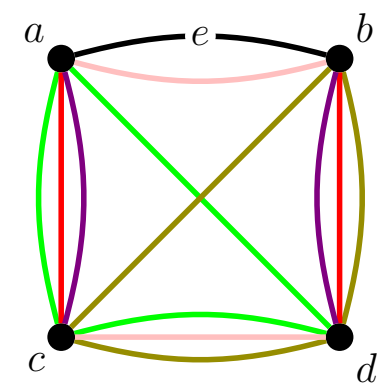

Figure 6.47: Betsy Ross configuration

1. the two green triangles are $a c d$ and $b c d$, and

2. the three red matchings are $(a b, c d)$, and $(a c, b d)$ twice.

Note that the edge $e=a b$ is in no $U_{2,4}$-restrictions but is in three non-clique three-point lines.

A representation is given in Figure 6.47.

Lemma 6.6.13. Let $C=(G, \mathcal{A})$ be a golden-mean augmented clique. Let $C^{\prime}=\left(G^{\prime}, \mathcal{A}^{\prime}\right)$ be a Betsy Ross configuration contained in $C$. Then $\mathcal{A}=\mathcal{A}^{\prime}$.

Proof. We use the labels in Figure 6.47. Let $X$ be an element of $\mathcal{A} \backslash \mathcal{A}^{\prime}$.

By Lemma 2.3.1, the Betsy Ross configuration is maximal on four vertices as it contains eleven elements, and all matroids that correspond to augmented cliques are simple by definition. Hence $X$ must use at least one vertex from $G \backslash G_{1}$. $X$ cannot use three or more vertices in $G \backslash G^{\prime}$, as then we would have a red matching edge in either $\mathcal{A}^{\prime}$ or $X$ that is vertex-disjoint from a green triangle, contradicting Lemma 6.3.4.

First, $X$ could be a green triangle $T$. If $T$ uses two vertices from $G \backslash G^{\prime}$, then $T$ cannot touch all red matching edges in $\mathcal{A}^{\prime}$ and hence this cannot happen by Lemma 6.3.4.

Hence $T$ can only use one vertex in $G \backslash G^{\prime}$. In this case, $T$ must contain an edge of $G^{\prime}$. As $T$ must touch every red matching edge from the red matchings in $\mathcal{A}^{\prime}$, the edge of $T$ from $G^{\prime}$ must be ad, up to symmetry. In this configuration, we are able to find a forbidden PM (Figure 6.12.3) using $T$, the green triangle $b c d$, and one of the $(a c, b d)$ red matchings.

Hence $X$ cannot be a green triangle.

So $X$ must be a red matching $R=\left(r_{1}, r_{2}\right)$. Consider the case that $R$ uses 
two vertices, $u$ and $v$, from $G \backslash G^{\prime}$. The edge $f=u v$ cannot be in $R$, as $f$ does not touch either green triangle in $\mathcal{A}^{\prime}$ and so is forbidden by Lemma 6.3.4. Hence $R=(u x, v y)$, where $\{x, y\} \subset\{a, b, c, d\}$. This leads to a forbidden TT (Figure 6.9.3), regardless of which vertices $x$ and $y$ are.

Hence there can only be one vertex $v$ in $G \backslash G^{\prime}$ that $R$ uses. As every edge of $R$ must touch every green triangle in $\mathcal{A}^{\prime}$ by Lemma 6.3.4, without loss of generality, $r_{1}=c v$.

This leaves three possible locations for $r_{2}$. If $r_{2}=a b$, then the two green triangles in $\mathcal{A}^{\prime}$ and $R$ form a forbidden KF (Figure 6.12.2). If $r_{2}=a d$, then the three red matchings from $\mathcal{A},(a b, c d),(a c, b d)$, and $(a d, c v)$ form a forbidden XG (Figure 6.11.1). Ergo $r_{2}=b d$. But now $(a b, c d)$ and $(b d, c v)$, and the green triangle $a c d$ form a forbidden HM (Figure 6.13.4) in $C$.

Hence $X$ cannot exist, and so $\mathcal{A}=\mathcal{A}^{\prime}$, as desired.

The name of the Betsy Ross configuration is not a coincidence. If $M$ is a golden-mean matroid with a spanning clique, and $M$ corresponds to an augmented clique that is isomorphic to the Betsy Ross configuration, then $M$ is the Betsy Ross matroid.

To show this, we use the function is_br() from Appendix 6.A.6. The function call is is_br $\operatorname{b}(4,[0,2,4],[3,4,5],[0,5],[0,5],[1$, 4] ]), which returns True, as desired.

Lemma 6.6.14. Let $M$ be a rank-r minimal counterexample to Theorem 6.1.4 such that $M$ is maximum-sized. There is no clique element e of $M$ such that $e$ is on three non-clique three-point lines and no $U_{2,4}$-restrictions.

Proof. Let $C=(G, \mathcal{A})$ be a golden-mean augmented clique associated with $M$. Assume that there is such a clique element $e$. Then $e$ must be on one of the four configurations considered in Section 6.4.3.

If $e$ is on the Betsy Ross configuration, then Lemma 6.6.13 implies that $|\mathcal{A}|=5$, and so

$$
\left(\begin{array}{c}
r+3 \\
2
\end{array}\right)-5 \leq \epsilon(M)=\left(\begin{array}{c}
r+1 \\
2
\end{array}\right)+5
$$

As $r \geq 5$, it is impossible for (6.6.1) to be true, so $M$ cannot be a counterexample to Theorem 6.1.4 in this case. 
Hence $e$ must be on a line-star, two-parallel, or matching-star configuration.

First, assume that $e$ is in a line-star configuration $C_{2}=\left(G_{2}, \mathcal{A}_{2}\right)$. Let $C_{1}=\left(G_{1}, \mathcal{A}_{1}\right)$ be a maximal semi-line-star configuration containing $C_{2}$ as a restriction. Lemmas 6.6.5 and 6.6.6 tell us that $\mathcal{A}=\mathcal{A}_{1}$. We now consider the maximum size $\epsilon(M)$ can have. $G$ has three identified vertices, $s, u$, and $v$. This leaves $r+1-3=r-2$ vertices, each of which determines up to two green triangles. Hence there are at most $2(r-2)$ elements in $\mathcal{A}$, for a maximum total of $\left(\begin{array}{c}r+1 \\ 2\end{array}\right)+2(r-2)$ elements.

Now assume that $e$ is in a two-parallel configuration $C_{2}=\left(G_{2}, \mathcal{A}_{2}\right)$. Let $C_{1}=\left(G_{1}, \mathcal{A}_{1}\right)$ be a maximal semi-line-star configuration containing $C_{2}$ as a restriction. Lemmas 6.6.8 and 6.6.9 tell us that $\mathcal{A}=\mathcal{A}_{1}$. We now consider the maximum size $\epsilon(M)$ can have. $G$ has three identified vertices, $a, b$, and c. This leaves $r+1-3=r-2$ vertices, each of which determines at most two red matchings. Hence there are no more than $2(r-2)$ elements in $\mathcal{A}$, with a maximum of $\left(\begin{array}{c}r+1 \\ 2\end{array}\right)+2(r-2)$ elements.

Finally assume that $e$ is in a matching-star configuration $C^{\prime}=\left(G^{\prime}, \mathcal{A}^{\prime}\right)$. Then Lemma 6.6.11 states that there are two possibilities for $\mathcal{A}^{\prime}$.

First, it could be that $\mathcal{A} \backslash \mathcal{A}^{\prime}$ consists of green triangles. In this case, part (i) of Lemma 6.6.11 applies. As before, we consider the maximum size $\epsilon(M)$ could have. In $G$, there are three distinguished vertices, $s, u$, and $v$. This leaves $r+1-3=r-2$ vertices, each of which determines at most one red matching in $\mathcal{A}^{\prime}$, and at most one green triangle in $\mathcal{A} \backslash \mathcal{A}^{\prime}$ for a maximum total of $r-2$ red matchings and $r-2$ green triangles. Hence the most points that $M$ could have is $\left(\begin{array}{c}r+1 \\ 2\end{array}\right)+2(r-2)$.

The other case is when $\mathcal{A} \backslash \mathcal{A}^{\prime}$ consists of red matchings. In this case, part (ii) of Lemma 6.6.11 applies. As before, there are three distinguished vertices, $s$, $u$, and $v$. Each vertex $x$ in $G \backslash\{s, u, v\}$ determines at most two red matchings, $(u v, s x)$ and $(s u, v x)$, and there are $r-2$ such vertices. Hence the maximum size of $\epsilon(M)$ is $\left(\begin{array}{c}r+1 \\ 2\end{array}\right)+2(r-2)$.

We know that if $M$ is a counterexample, then $\epsilon(M) \geq\left(\begin{array}{c}r+3 \\ 2\end{array}\right)-5$. So if any of our configurations are to be a counterexample then

$$
\left(\begin{array}{c}
r+1 \\
2
\end{array}\right)+2(r-2) \geq\left(\begin{array}{c}
r+3 \\
2
\end{array}\right)-5
$$


must be true. However, (6.6.2) implies that $\frac{5}{2} r-4 \geq \frac{5}{2} r-2$, which is false. So our configurations cannot be counterexamples.

Therefore no such $e$ can exist.

Corollary 6.6.15. Let $M$ be a rank-r minimal counterexample to Theorem 6.1.4 such that $M$ is maximum-sized. Then every clique element that is not in a $U_{2,4}$-restriction must be on exactly two non-clique three-point lines.

Proof. Let $e$ be a clique element of $M$ that is not in any $U_{2,4}$-restrictions. Since $M$ is a minimal counterexample, we have that $\epsilon(M) \geq\left(\begin{array}{c}r+3 \\ 2\end{array}\right)-5$, and that $\epsilon(M / e) \leq\left(\begin{array}{c}r+2 \\ 3\end{array}\right)-5$. Hence

$$
\epsilon(M)-\epsilon(M / e) \geq r+2
$$

As $e$ is not in any $U_{2,4}$-restrictions, it follows from (6.6.3) that $e$ is on at least $r+1$ three-point lines. Therefore $e$ must be on at least two non-clique threepoint lines. However, Lemma 6.6.14 states that $e$ can be on no more than two non-clique three-point lines. Hence $e$ must be on exactly two non-clique three-point lines.

Corollary 6.6.16. Let $M$ be a rank-r minimal counterexample to Theorem 6.1.4 such that $M$ is maximum-sized, and let e be a clique element of $M$ that is on no $U_{2,4}$-restrictions. Then $\mathrm{si}(M / e)$ is isomorphic to a member of $\mathscr{G}_{r-1}$.

We now construct all augmented clique representations for all members of $\mathscr{G}_{r}$.

Lemma 6.6.17. Let $M$ be isomorphic to $T_{r}^{2}$ for some $r \geq 3$. Let $(G, \mathcal{A})$ be a golden-mean augmented clique associated with $M$. Then $\mathcal{A}$ consists of $2(r-1)$ green triangles. There is an edge e of $G$ such that every green triangle in $\mathcal{A}$ contains e. For every vertex $v$ of $G$ not incident with e, there are exactly two green triangles in $\mathcal{A}$ that contain $e$ and $v$.

Proof. Let $H$ be the weighted directed graph that corresponds to a $\mathbb{G}$-graphic representation of $M$, as described on Page 32. The loop in $H$ that is incident with all non-trivial parallel classes is the acme of $M$. Let $a$ be this loop, and 


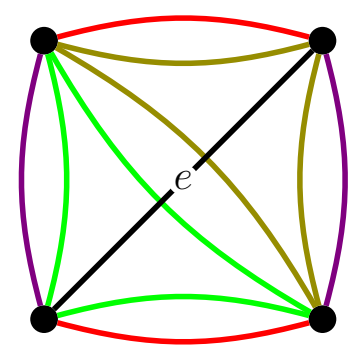

Figure 6.48: Five-point line with four non-clique elements

assume that $a$ is not in $G$. There must be an element in $G$ that is contained in a leg of $M$, for otherwise $G$ cannot be spanning. Let $b$ be such an element. Any permutation of the elements of a leg that fixes the acme, when applied to all the legs of $M$ simultaneously, is an automorphism of $M$. Therefore we can assume that $b$ is a loop in $H$. Now $b$ is contained in $r-1$ triangles of $G$. Since $b$ is contained in $r-1$ long lines in $M$, it follows that $\mathrm{cl}_{M}(\{a, b\})$ contains three elements of $G$. Let $\{b, c, d\}$ be those three elements, so that in $H$, the edges that $c$ and $d$ correspond to are incident with both $a$ and $b$. One of the other triangles of $G$ that contains $b$ also contains $f$, another loop of $H$, as well as the edge of $H$ that is incident with $b$ and $d$. Since $\{b, c, d\}$ is a triangle of $G$, and $\{b, f\}$ is contained in a triangle of $G$, it follows that $f$ is contained in a triangle of $G$ with either $c$ or $d$. But consideration of $H$ shows that no such line exists.

Now we know that $G$ contains $a$, the acme of $M$. By again applying an automorphism of $M$, we can assume that $G$ contains $b$, which is another loop in $H$. Since $b$ is contained in $r-1$ lines in $G$, an inspection of $H$ shows that all loops of $H$ must be in $G$, as well as precisely one edge between each pair of vertices. Now the elements of $M$ not in $G$ correspond to two edges from each non-trivial parallel class of $H$. We see that each triangle of $G$ that contains $a$ spans two elements not in $G$, and the result follows easily.

Lemma 6.6.18. Let $M$ be a simple golden-mean matroid of rank at least four with a spanning clique, and assume that $(G, \mathcal{A})$ is a corresponding golden-mean augmented clique. Let $\ell$ be a five-point line of $M$. Then there is a $K_{5}$ subgraph of $G$ that contains all members of $\mathcal{A}$ that correspond to elements of $\ell$. 
Proof. It is clear that $\ell$ contains no more than three edges from $G$. If $\ell$ contains exactly three edges from $G$, then by the definition of green elements, both non-clique elements of $\ell$ must be green triangles, and furthermore, they must have the same vertex set, so there is nothing left to prove. Likewise, if $\ell$ contains exactly two elements from $G$, then the three non-clique elements of $\ell$ must be three red matchings sharing the same vertex set, and we are done.

Now assume that $\ell$ contains four non-clique elements, $X_{1}, \ldots, X_{4}$, as well as $e$, a clique element. Then the four elements $e, X_{x}, X_{y}$, and $X_{z}$ must form a $U_{2,4}$-restriction for all $\{x, y, z\} \subset\{1,2,3,4\}$. All such $U_{2,4}$-restrictions were discovered in Section 6.5.3.5. From this, we can see that the only way to form a five-point line with four non-clique elements is the configuration shown in Figure 6.48, and again we are done.

Hence we assume that $\ell$ consists entirely of non-clique elements. Let the elements of $\ell$ be $X_{1}, \ldots, X_{5}$. Note that any long line containing two or more elements of $\ell$ must only contain elements of $\ell$. Hence, in particular, any two elements of $\ell$ must not be on a long line with a clique element.

Assume that $X_{1}$ and $X_{2}$ are green triangles. Then, using the computer checks from Section 6.4.1.2 and the observation above, we can see that $X_{1}$ and $X_{2}$ must join at a vertex $v$. Furthermore, any two green triangles that are in $\ell$ must meet at a vertex. Now consider $X_{3}$. If $X_{3}$ is a green triangle, then it must meet both $X_{1}$ and $X_{2}$ at a single vertex. Hence $X_{1}, X_{2}$, and $X_{3}$ must form either a RA (Figure 6.8.6) or a TB (Figure 6.8.4), both of which are forbidden. So $X_{3}$ must be a red matching.

By Lemma 6.3.4, both edges of $X_{3}$ must touch both $X_{1}$ and $X_{2}$. This means that the edges of $X_{3}$ must either be incident with $v$, or be of the form $x_{1} x_{2}$, where $x_{i} \in X_{i} \backslash\{v\}$. Assume that one edge of $X_{3}$ is incident with $v$. Then $X_{1}, X_{2}$, and $X_{3}$ form either a SR (Figure 6.13.5) or a PM (Figure 6.12.3), both of which are forbidden. Hence $X_{3}$, and, by an identical argument, $X_{4}$ and $X_{5}$, must be contained in the $K_{5}$ spanned by $X_{1}$ and $X_{2}$, as desired.

Therefore we assume that $\ell$ contains at most one green element. So we can assume that $X_{1}, \ldots, X_{4}$ are all red matchings. By considering the computer checks from Section 6.4.1.3, we can assume that $X_{i}$ and $X_{j}$ form an alternating path, for all $\{i, j\} \subset\{1,2,3,4\}$. Assume that $X_{5}$ is a green triangle. 
Then, by the computer checks in Section 6.4.1.4, $X_{5}$ must form the configuration shown in Figure 6.16 .2 with each of $X_{1}, \ldots, X_{4}$. Hence $X_{1}, X_{2}$, and $X_{5}$ must form either a FS (Figure 6.13.2) or a BP (Figure 6.13.6), both of which are forbidden. Therefore $X_{5}$ must be a red matching.

Now consider the three red matchings $X_{1}, X_{2}$, and $X_{3}$, and assume that $X_{3}$ is not contained within the $K_{5}$ spanned by $X_{1} \cup X_{2}$. As $X_{3}$ must form an alternating path with both $X_{1}$ and $X_{2}$, it must be that $X_{1}, X_{2}$, and $X_{3}$ form a forbidden DI (Figure 6.11.6). Hence $X_{3}$ must be contained within the $K_{5}$ spanned by $X_{1} \cup X_{2}$, and the result follows.

Lemma 6.6.19. Let $M$ be isomorphic to either $G_{r}$ or $H P_{r}$ for $r \geq 4$, and let $C=(G, \mathcal{A})$ be a corresponding golden-mean augmented clique. Then $C$ is one of the following three configurations.

(i) C consists of a line-star configuration (see Definition 6.6.4) that spans every vertex of $G$ with the addition of two green triangles, both on the vertices suv. In this case, $M \cong G_{r}$.

(ii) $C$ consists of a two-parallel configuration (see Definition 6.6.7) that spans every vertex of $G$ with the addition of two green triangles, both on the vertices abc. In this case, $M \cong H P_{r}$.

(iii) $C$ consists of a matching-star configuration (see Definition 6.6.10) that spans every vertex of $G$ with the addition of all possible green triangles as described in part (i) of Lemma 6.6.11, as well as two additional green triangles, both on the vertices suv. In this case, $M \cong G_{r}$.

Proof. We first show that the lemma is true for low rank.

Sublemma 6.6.19.1. The lemma is true when $4 \leq r \leq 5$.

Subproof. We prove this by case analysis. The code in Appendix 6.A.7 creates eighteen augmented cliques at both rank four and rank five. We then create each augmented clique by hand, and the sublemma is verified.

Now assume that the lemma is false, and that $r$ is is the smallest value for which the lemma fails. Note that, by Sublemma 6.6.19, $r \geq 6$, and hence $G$ has at least seven vertices. Let $\ell$ be the unique five-point line of $M$, and recall that $G$ is an arbitrary spanning clique of $M$. We start by showing that $\ell$ contains exactly three edges of $G$. 
Sublemma 6.6.19.2. The unique five-point line $\ell$ of $M$ contains exactly two non-clique elements.

Subproof. Assume that this sublemma is false. It is clear that $\ell$ contains at least two non-clique elements. If $\ell$ contains exactly three non-clique elements, then these three non-clique elements are spanned by two clique elements, and hence are distinct red matchings with identical edge sets. However, this is a forbidden TM (Figure 6.10.2).

Hence $\ell$ contains at least four non-clique elements. By Lemma 6.6.18, there is a $K_{5}$-subgraph $K$ of $G$ that contains all members of $\mathcal{A}$ corresponding to elements of $\ell$. Let $e$ be an edge of $G \backslash K$ such that $e$ is not incident with any edge of $G$ that is in $\ell$. Note that $e$ exists, as at most one element of $\ell$ is an edge of $G$, and this edge must be spanned by $K$, and $G$ has at least seven vertices.

By our choice of $e$, there is no triangle of $M$ consisting of $e$, an edge of $G$ that is not in $\ell$, and an edge of $G$ that is in $\ell$. Assume that $T$ is a triangle of $M$ containing $e$, an edge $e^{\prime}$ of $G$ not in $\ell$, and an $l$ that is in $\ell$. Then $l$ corresponds to a member of $\mathcal{A}$. Then $l$ is spanned by the edges $e$ and $e^{\prime}$, so the member of $\mathcal{A}$ corresponding to $l$ contains the edge $e$, which is contradictory, since this member of $\mathcal{A}$ should be contained in $K$. Hence, in $M / e$, no parallel class contains an element of $\ell$ and an edge of $G$.

Now we can assume that $\ell$ is a five-point line in $\operatorname{si}(M / e)$, and we can assume that $\operatorname{si}(G / e)$ is a spanning clique of $\operatorname{si}(M / e)$ that does not contain three points in $L$. However, since $e$ is not in five-point line of $M$, Corollary 2.2.2 tells us that $\operatorname{si}(M / e)$ is isomorphic to $G_{r-1}$ or $H P_{r-1}$.

Now we can apply induction, which leads to a contradiction, since in the augmented cliques described in the statement of the lemma, the five-point line contains exactly two non-clique elements.

Hence $G$ contains exactly three elements of $\ell$. Let $T$ be the triangle of $G$ formed by these three elements. Since $\left|\mathrm{cl}_{M}(T)\right|=5$, we see that $\mathcal{A}$ contains two copies of $T$. Let these copies be $T_{1}$ and $T_{2}$, and let $t_{1}$ and $t_{2}$ be the corresponding elements of $\ell$. Consider the golden-mean augmented clique $C^{\prime}=\left(G, \mathcal{A}^{\prime}\right)$, where $\mathcal{A}^{\prime}=\mathcal{A} \backslash\left\{T_{1}, T_{2}\right\}$. Note that $C^{\prime}$ corresponds to the golden-mean matroid $M^{\prime}=M \backslash t_{1} \backslash t_{2}$. By inspecting the matrices of $G_{r}$ 
(Figure 2.1.2) and $H P_{r}$ (Figure 2.1.3), we note that there exists an edge $x$ in $T$, such that $x$ is in no $U_{2,4}$-restrictions in $M^{\prime}$, and $x$ is contained in $2(r-2)$ three-point lines in $M^{\prime}$. As $r \geq 6$ (Sublemma 6.6.19.1 covers the lower ranks), this means that $x$ is on at least three non-clique three-point lines, and we can apply the case analysis from Section 6.4.3, to get that $C^{\prime}$ is one of the configurations given in Figure 6.34 (with the exception of the Betsy Ross, as $r \geq 6)$.

We now consider $C$, which is the augmented clique $C^{\prime}$ with the addition of two green triangles, $T_{1}$ and $T_{2}$. Note that $T_{1}$ and $T_{2}$ have the same vertex set $T$.

First, we assume that $C^{\prime}$ is a line-star configuration (Definition 6.6.4). Using the labels from Figure 6.44, if $T=\{s, u, v\}$, then we are done. Hence, without loss of generality, $T=\left\{l_{1}, u, v\right\}$. In this case, the three green triangles $T_{1}$, $s u l_{1}$, and $s v l_{2}$ form a forbidden OM (Figure 6.8.2). Therefore $T=\{s, u, v\}$ and case (i) holds. The additional remark that $M \cong G_{r}$ follows from the fact that there are green elements in $M$, which equate to $U_{2,4}$-restrictions.

Now we assume that $C^{\prime}$ is a two-parallel configuration (Definition 6.6.7). Using the labels from Figure 6.45, if $T=\{a, b, c\}$, then we are done. So, without loss of generality, we can assume that $T=\left\{a, c, v_{1}\right\}$. In this case, the two green triangles $T_{1}$ and $T_{2}$, along with the red matching $\left(a b, c v_{2}\right)$, form a CC (Figure 6.12.4), which is forbidden. So $T=\{a, b, c\}$ and case (ii) holds. The additional remark that $M \cong H P_{r}$ follows from the fact that there are no green elements in $M$ with the exception of $t_{1}$ and $t_{2}$.

Finally we assume that $C^{\prime}$ is a matching-star configuration (Definition 6.6.10). We can apply either case (i) or case (ii) of Lemma 6.6.11 to $C^{\prime}$. If we apply case (ii), we can see that $C^{\prime}$ is contained within a two-parallel configuration, which was considered above. Hence we must apply case (i). Using the labels from Figure 6.46, if $T=\{s, u, v\}$, then we are done. Thus, without loss of generality, $T=\left\{u, v, m_{1}\right\}$. Now the green triangle $T_{1}$ does not touch the red matching edge $s m_{2}$, which is a contradiction to Lemma 6.3.4. Hence $T=\{s, u, v\}$, and case (iii) holds. The additional remark that $M \cong G_{r}$ follows from the fact that there are at least three green elements in $M$, which equate to $U_{2,4}$-restrictions. 


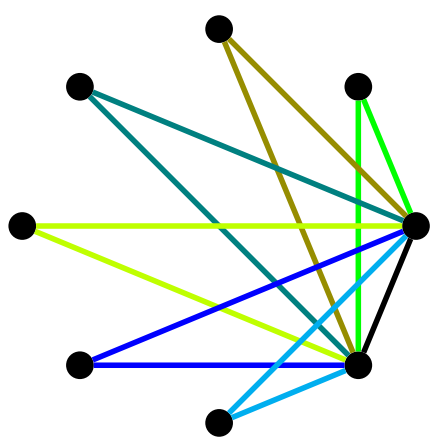

6.49.1: $\mathfrak{W}$ (Lemma 6.6.17)

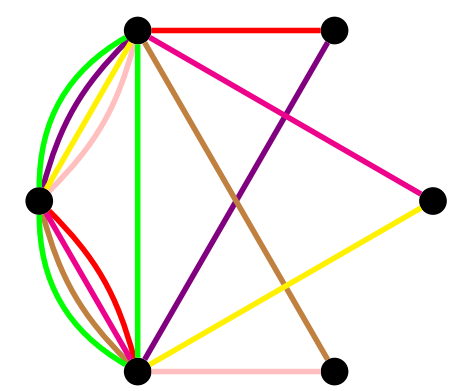

6.49.3: $\mathfrak{Y}$ (Lemma 6.6.19 (ii))

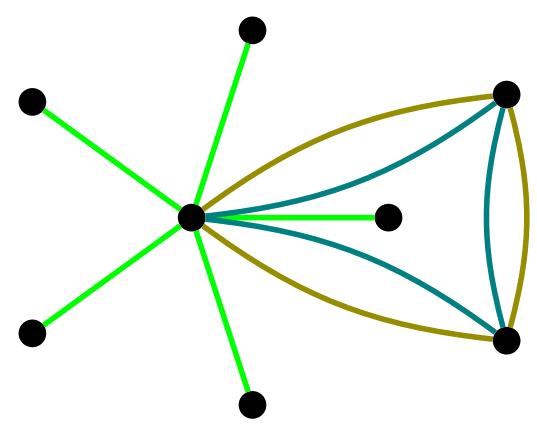

6.49.2: $\mathfrak{X}$ (Lemma 6.6.19 (i))

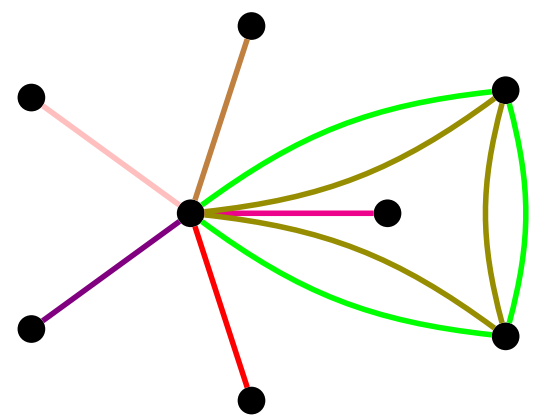

6.49.4: 3 (Lemma 6.6.19 (iii))

Figure 6.49: Schematics of the four possible outcomes for $C_{e}$ and $C_{f}$

We can now prove Theorem 6.1.4. Let $M$ be a rank- $r$ counterexample to Theorem 6.1.4 with an augmented clique representation $C=(G, \mathcal{A})$ such that $M$ is maximum-sized. Let $e$ be a clique element of $M$ that is in no $U_{2,4^{-}}$ restrictions. Then Corollary 6.6.16 tells us that $M_{e}=\mathrm{si}(M / e)$ is isomorphic to a member of $\mathscr{G}_{r-1}$.

Let $C_{e}=\left(G_{e}, \mathcal{A}_{e}\right)$ be an augmented clique corresponding to $M_{e}$. Now, Lemmas 6.6.17 and 6.6.19 tell us that $C_{e}$ is one of the four configurations shown in Figure 6.49.

Let $f$ be an edge of $G_{e}$ that is not in any $U_{2,4}$-restrictions in $M_{e}$. If $f$ were in a $U_{2,4}$-restriction in $M$, this restriction would have to contain $e$, which is a contradiction. Hence $f$ is not in any $U_{2,4}$-restrictions in $M$, and we can apply Corollary 6.6.16 to find that $M_{f}=\mathrm{si}(M / f)$ is also isomorphic to a member of $\mathscr{G}_{r-1}$.

Let $C_{f}=\left(G_{f}, \mathcal{A}_{f}\right)$ be an augmented clique corresponding to $M_{f}$. Now, Lemmas 6.6.17 and 6.6.19 tell us that $C_{f}$ is one of the four configurations shown in Figure 6.49. 
First, we assume that $C_{e}$ is a $\mathfrak{W}$ (Figure 6.49.1). Then we can select $f$ so that it does not touch the vertex identified with $e$ in $C_{e}$ and is not in any green triangles. Now, upon considering the possible members of $\mathcal{A}_{f}$ and Lemma 6.3.2, we note that $C_{f}$ must also be a $\mathfrak{B}$, and hence $C$ is a $\mathfrak{W}$. We now construct a $G F(4)$ matrix for $M$. Let $D_{n}^{x}$ be the $n \times\left(\begin{array}{l}n \\ 2\end{array}\right)$ matrix whose entries consist of all $n$-tuples with two non-zero entries, with the first being 1 and the second being $x$. When we consider the star of $G$ where the core is a vertex that is touched by all green triangles of $\mathcal{A}$, it is not too hard to see that $M$ must be represented over $G F(4)$ by a matrix of the following form.

$$
\left[I_{r}\left|D_{r}^{1}\right| D_{r}^{\alpha} \mid D_{r}^{\alpha^{2}}\right]
$$

This matrix is scaling-equivalent to the $G F(4)$ matrix for $T_{r}^{2}$ given in Figure 2.5.1, and hence $M$ is isomorphic to $T_{r}^{2}$.

We now assume that $C_{e}$ is a $\mathfrak{X}$ (Figure 6.49.2). Then we can select $f$ so that it does not touch the vertex identified with $e$ in $C_{e}$ and is not in any green triangles. Now, upon considering the possible members of $\mathcal{A}_{f}$ and Lemma 6.3.2, we note that $C_{f}$ must also be a $\mathfrak{X}$, and hence $C$ is a $\mathfrak{X}$. We now construct a $G F(4)$ matrix for $M$. When we consider the star of $G$ where the core is the vertex of $G$ that is the core of the star from the underlying line-star configuration, it is not too hard to see that $M$ must be represented over $G F(4)$ by a matrix of the following form.

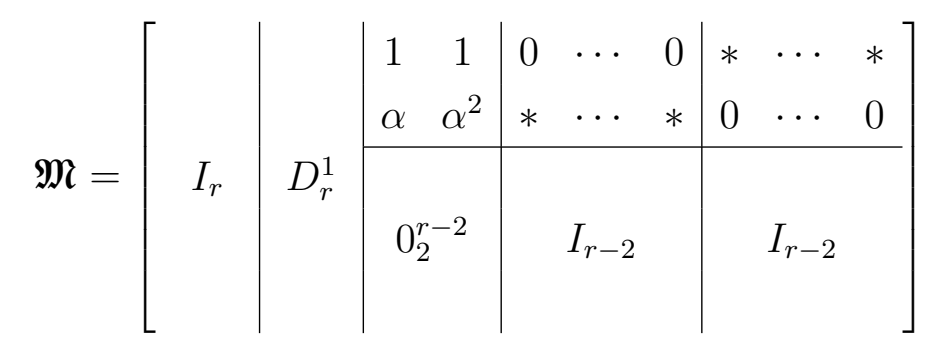

where each $*$ is either $\alpha$ or $\alpha^{2}$. Now, by selecting the correct rows and columns, we can find a rank-three submatrix of $\mathfrak{M}$ for each $*$ in the fourth block of $\mathfrak{M}$. This submatrix must be of the form

$$
\left[\begin{array}{cccccccccc}
1 & 0 & 0 & 1 & 1 & 0 & 1 & 1 & 0 & *_{2} \\
0 & 1 & 0 & 1 & 0 & 1 & \alpha & \alpha^{2} & *_{1} & 0 \\
0 & 0 & 1 & 0 & 1 & 1 & 0 & 0 & 1 & 1
\end{array}\right] .
$$


This matrix represents a rank-three golden-mean matroid with ten elements, and hence, by Lemma 2.3.1, must be isomorphic to either $T_{3}^{2}, G_{3}, H P_{3}, Y_{10}$, $S_{10}$ or $B R \backslash p$. As there is a five-point line, we can further restrict this list of possible matroids to $T_{3}^{2}, G_{3}$, or $H_{3}$. As there are four-point lines, this submatrix must therefore represent $G_{3}$ over $G F(4)$. Now, by uniqueness of representation, we can conclude that $*_{1}=*_{2}=\alpha$.

By repeating this argument, we can discover that

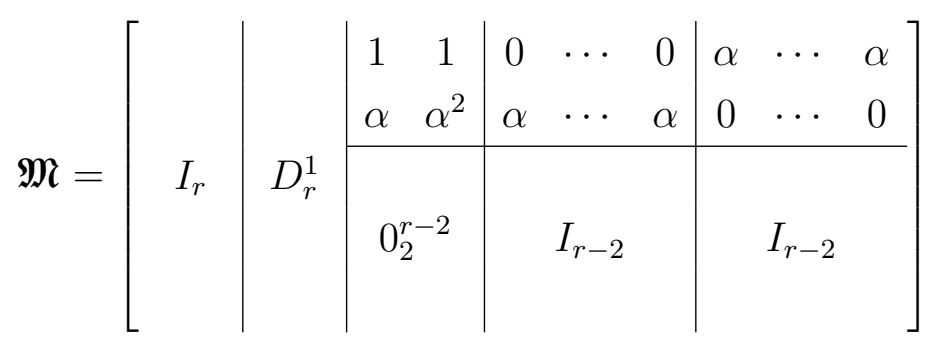

which is the $G F(4)$ matrix for $G_{r}$ given in Figure 2.5.2, and hence $M \cong G_{r}$. Next, we consider the case where $C_{e}$ is a $\mathfrak{g}$ (Figure 6.49.3). Then we can select $f$ so that it does not touch the vertex identified with $e$ in $C_{e}$ and is not in any green triangles or red matchings. Now, upon considering the possible members of $\mathcal{A}_{f}$ and Lemma 6.3.2, we note that $C_{f}$ must also be a $\mathfrak{Y}$, and hence $C$ is a $\mathfrak{Y}$. We now construct a $G F(4)$ matrix for $M$. When we consider the star of $G$ where the core is the vertex of $G$ that is incident with both sets of parallel classes of red matchings, it is not too hard to see that $M$ must be represented over $G F(4)$ by a matrix of the following form.

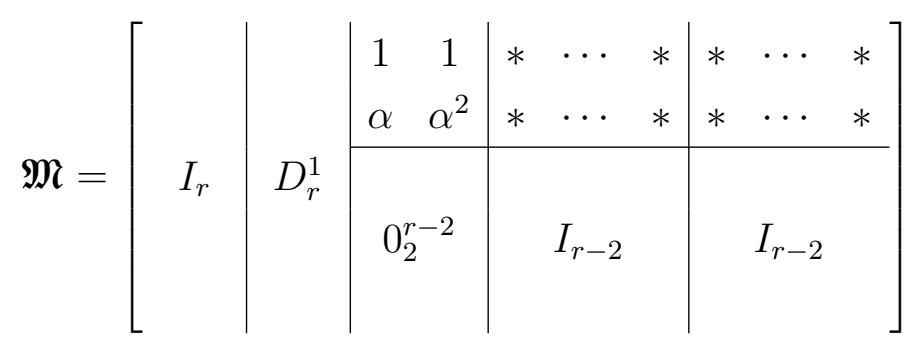

where each $*$ is a non-zero element of $G F(4)$. Now, by selecting the correct rows and columns, we can find a rank-three submatrix of $\mathfrak{M}$ for each column 
from the fourth block of $\mathfrak{M}$. This submatrix must be of the form

$$
\left[\begin{array}{cccccccccc}
1 & 0 & 0 & 1 & 1 & 0 & 1 & 1 & *_{1} & *_{3} \\
0 & 1 & 0 & 1 & 0 & 1 & \alpha & \alpha^{2} & *_{2} & *_{4} \\
0 & 0 & 1 & 0 & 1 & 1 & 0 & 0 & 1 & 1
\end{array}\right] .
$$

This matrix represents a rank-three golden-mean matroid with ten elements, and hence, by Lemma 2.3.1, must be isomorphic to either $T_{3}^{2}, G_{3}, \mathrm{HP}_{3}$, $Y_{10}, S_{10}$ or $B R \backslash p$. As there is a five-point line, we can further restrict this list of possible matroids to $T_{3}^{2}, G_{3}$, or $H P_{3}$. As there are no further very long lines, this submatrix must therefore represent $H P_{3}$ over $G F(4)$. Now, by uniqueness of representation, we can conclude that $*_{1}=*_{4}=\alpha$ and $*_{2}=*_{3}=1$.

By repeating this argument, we can discover that

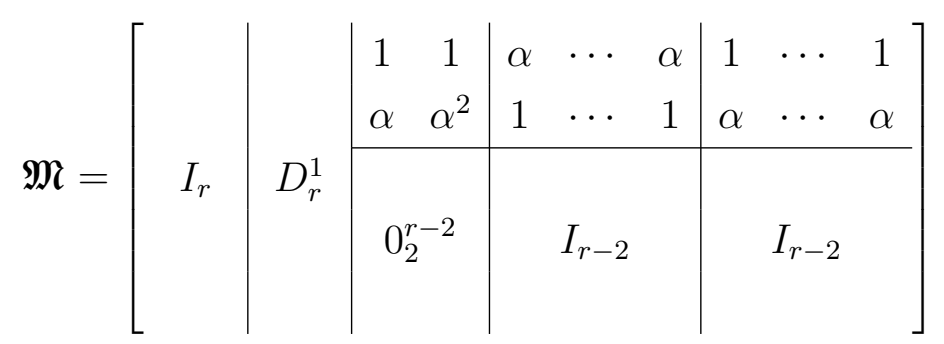

which is scaling-equivalent to the $G F(4)$ matrix for $H P_{r}$ given in Figure 2.5.3, and hence $M \cong H P_{r}$.

Finally, we assume that $C_{e}$ is a $\mathbf{Z}$ (Figure 6.49.4). Then we can select $f$ so that it does not touch the vertex identified with $e$ in $C_{e}$ and is not in any green triangles or red matchings. Now, upon considering the possible members of $\mathcal{A}_{f}$ and Lemma 6.3.2, we note that $C_{f}$ must also be a $\boldsymbol{Z}$, and hence $C$ is a $\mathbf{3}$. We now construct a $G F(4)$ matrix for $M$. When we consider the star of $G$ where the core is the vertex of $G$ that is the core of the star from the underlying matching-star configuration, it is not too hard to see 
that $M$ must be represented over $G F(4)$ by a matrix of the following form.

$$
\mathfrak{M}=\left[\begin{array}{l|l|cc|ccc|ccc}
1 & 1 & 0 & \cdots & 0 & \star & \cdots & \star \\
I_{r} & D_{r}^{1} & \alpha & \alpha^{2} & * & \cdots & * & \star & \cdots & \star \\
\cline { 3 - 8 } & & 0_{2}^{r-2} & & I_{r-2} & & & I_{r-2} \\
& & & & & & & &
\end{array}\right]
$$

where each $*$ is either $\alpha$ or $\alpha^{2}$, and each $\star$ is a non-zero element of $G F(4)$ with the added condition that two $\star s$ in the same column have the same value. Now, by selecting the correct rows and columns, we can find a rank-three submatrix of $\mathfrak{M}$ for each $*$ in the fourth block of $\mathfrak{M}$. This submatrix must be of the form

$$
\left[\begin{array}{cccccccccc}
1 & 0 & 0 & 1 & 1 & 0 & 1 & 1 & 0 & \star \\
0 & 1 & 0 & 1 & 0 & 1 & \alpha & \alpha^{2} & * & \star \\
0 & 0 & 1 & 0 & 1 & 1 & 0 & 0 & 1 & 1
\end{array}\right] .
$$

This matrix represents a rank-three golden-mean matroid with ten elements, and hence, by Lemma 2.3.1, must be isomorphic to either $T_{3}^{2}, G_{3}, H P_{3}, Y_{10}$, $S_{10}$ or $B R \backslash p$. As there is a five-point line, we can further restrict this list of possible matroids to $T_{3}^{2}, G_{3}$, or $H P_{3}$. As there are also four-point lines, this submatrix must therefore represent $G_{3}$ over $G F(4)$. Now, by uniqueness of representation, we can conclude that $*=\star=\alpha^{2}$.

By repeating this argument, we can discover that

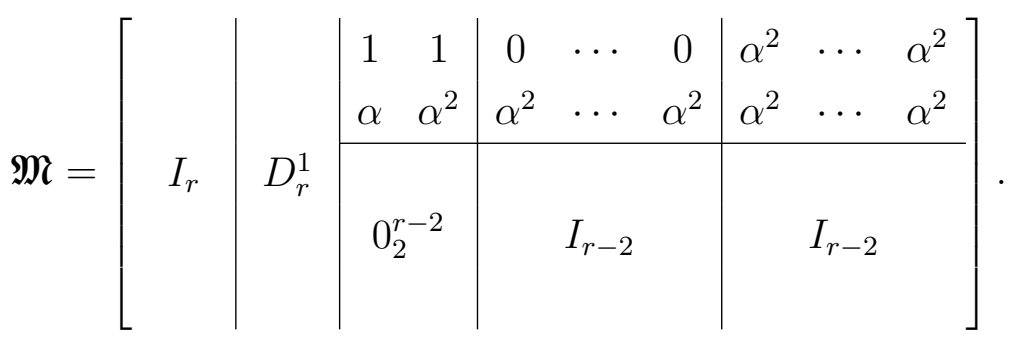

Let $\mathfrak{N}$ be the $G F(4)$ matrix of $G_{r}$ from Figure 2.5.2. Add the first row of $\mathfrak{N}$ to the second row, and add every other row of $\mathfrak{N}$ scaled by $\alpha$ to the second row of $\mathfrak{N}$. Now $\mathfrak{N}$ is scaling-equivalent to $\mathfrak{M}$, and thus $M \cong G_{r}$. 


\subsection{Future Work}

It is anticipated that our Theorem 6.1.4, when combined with the following theorem due to Geelen and Nelson (personal correspondence) will lead to a proof of Conjecture 1.2.3 for matroids of sufficiently high rank. In this section, we outline how we anticipate this combination will occur.

Theorem 6.7.1. Let $\mathcal{M}$ be a quadratically dense minor-closed class of matroids and let $p(x)$ be a real quadratic polynomial with positive leading coefficient. If $h_{\mathcal{M}}(n)>p(n)$ for infinitely many $n \in \mathbb{Z}^{+}$, then for all $r, s \in \mathbb{Z}^{+}$ there exists $M \in \mathcal{M}$ satisfying $\epsilon(M)>p(r(M))$ and $r(M) \geq r$ such that either

(1) $M$ has a spanning clique restriction, or

(2) $M$ is vertically s-connected and there is an s-element independent set $S$ of $M$ so that $\epsilon(M)-\epsilon(M / e)>p(r(M))-p(r(M)-1)$ for each $e \in S$.

If Conjecture 1.2.3 is false, then the conditions of Theorem 6.7.1 are met. Theorem 6.1.4 shows that outcome (1) cannot occur, and hence outcome (2) must occur. To show that outcome (2) is also impossible, we need to apply the following lemmas, both due to Geelen and Nelson (personal correspondence).

Lemma 6.7.2. Let $k \in \mathbb{Z}^{+}$, let $M$ be a matroid and let $N$ be a minor of $M$ such that $\mathcal{T}_{k}(N)$ is a tangle. If $X \subseteq E(M)$ is contained in a $\mathcal{T}_{k}(M, N)$ small set, then there is a minor $M^{\prime}$ of $M$ such that $M^{\prime}|X=M| X, M^{\prime}$ has $N$ as a minor, and $X$ is contained in a $\mathcal{T}_{k}\left(M^{\prime}, N\right)$-small set $X^{\prime}$ such that $E\left(M^{\prime}\right)=E(N) \cup X^{\prime}$ and $\lambda_{M^{\prime}}\left(X^{\prime}\right)=r_{\mathfrak{T}_{k}\left(M^{\prime}, N\right)}(X)=r_{\mathfrak{T}_{k}(M, N)}(X)$.

Lemma 6.7.3. There is a function $f: \mathbb{Z}^{2} \rightarrow \mathbb{Z}$ so that, for all $m, n, l, t \in \mathbb{Z}$ with $m>t \geq 0, l \geq 2$, and $n \geq f(m, l)$, if $M \in \mathcal{U}(l)$ has an $M\left(K_{n+1}\right)$-minor $N$ with corresponding tangle $\mathcal{T}=\mathcal{T}_{\lceil 2 n / 3\rceil}(M, N)$ and $X \subseteq E(M)$ satisfies $r_{\mathcal{T}}(X)=t$, then $M$ has a minor $M^{\prime}$ with an $M\left(K_{m+1}\right)$-restriction $R$ so that $X \cap E(R)=\varnothing, M^{\prime}|X=M| X, E\left(M^{\prime}\right)=E(R) \cup X$ and $\lambda_{M^{\prime}}(X)=t$.

Using these two lemmas, we are able to glue the $s$-element independent set from outcome (2) of Theorem 6.7.1 onto a spanning clique, where we anticipate that our techniques developed in the proof of Theorem 6.1.4 will be able to show that this case is also contradictory. 


\section{Appendix 6.A Code}

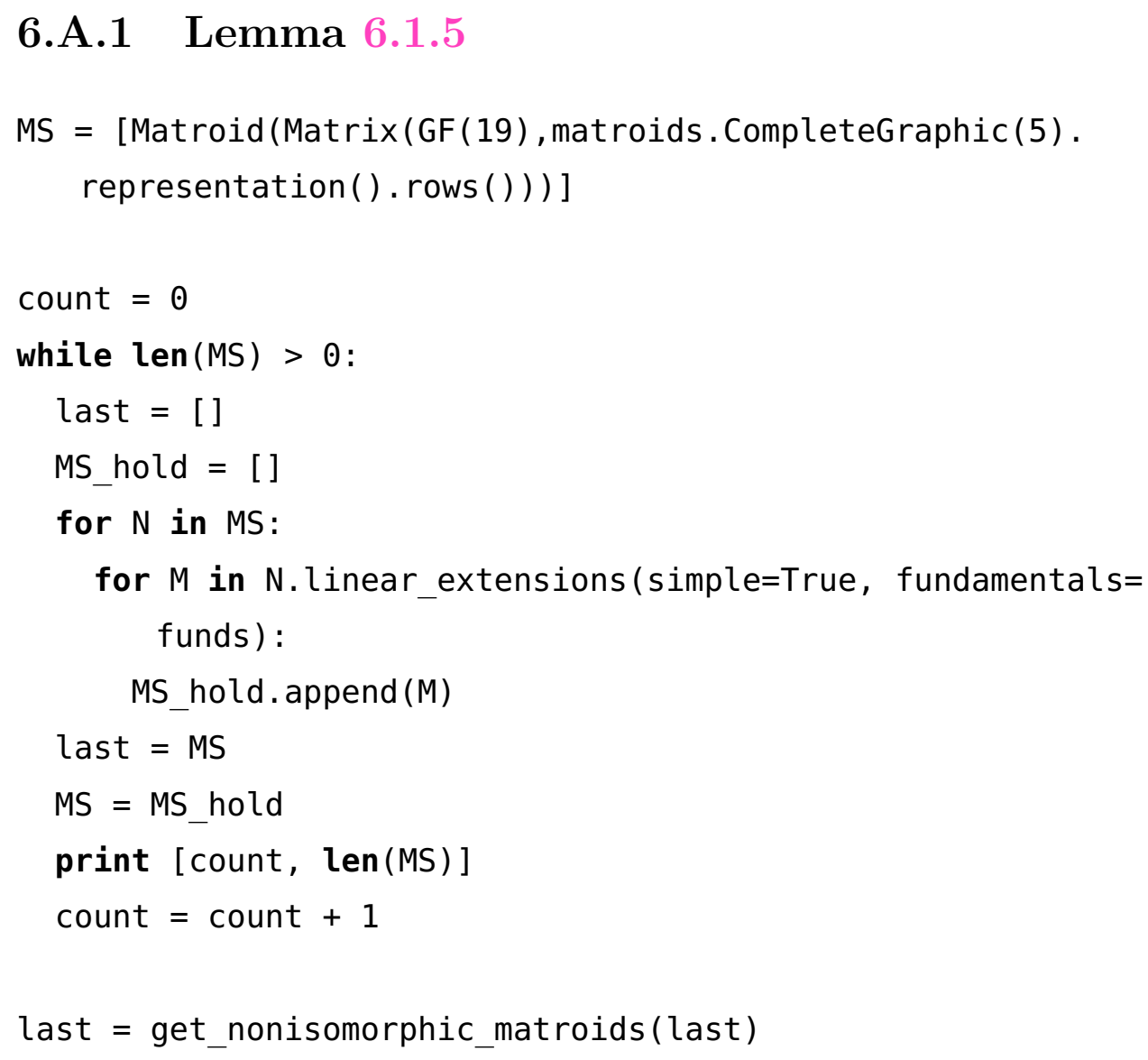

This code starts from $M\left(K_{5}\right)$ and constructs all golden-mean matroids (over $G F(19))$ that have $M\left(K_{5}\right)$ as a restriction. Once there are no more possible extensions, it creates a list, last of matroids that are maximum-sized.

Upon running this code (which takes a long time), we discover that Lemma 6.1.5 is indeed true. That is, Theorem 6.1 .4 is true when $r=4$.

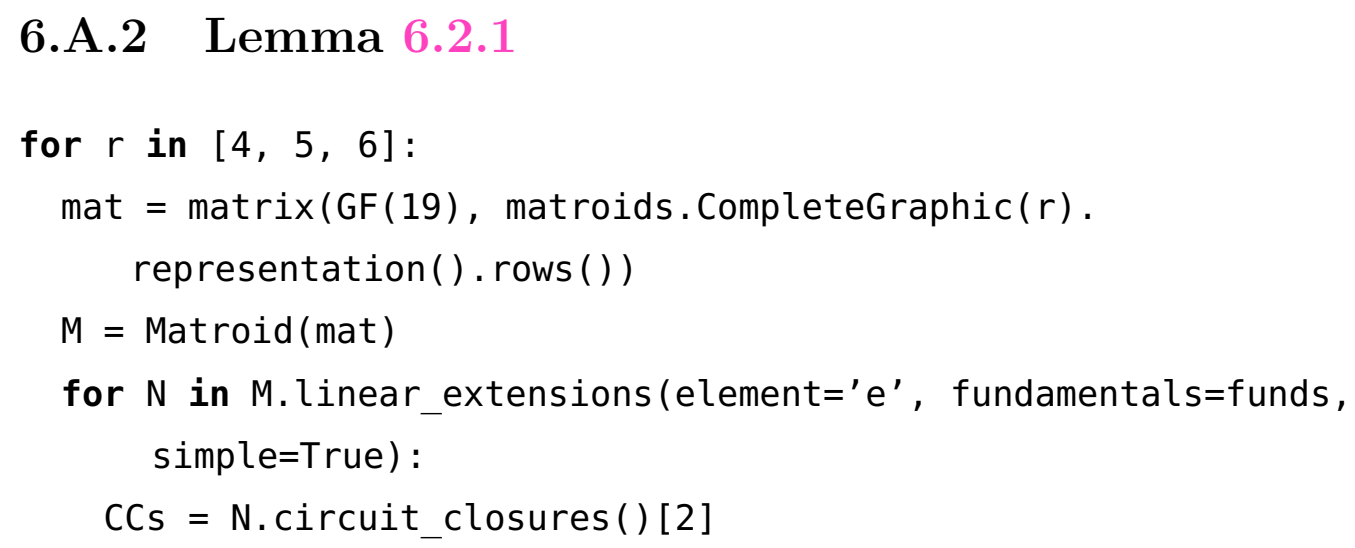




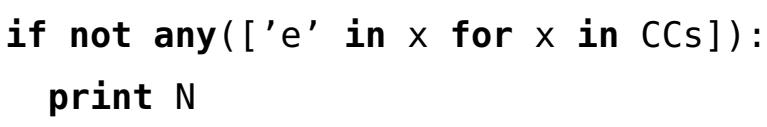

This code is used to prove Lemma 6.2.1. We construct all golden-mean single element extensions of $M\left(K_{\mathrm{r}}\right)$ for $r$ in $[4,5,6]$. If there were any counterexamples to the lemma, then $e$ would not be in a long line of the constructed matroid. This code prints out any matroids that give counterexamples, and nothing is output, so no counterexamples exist.

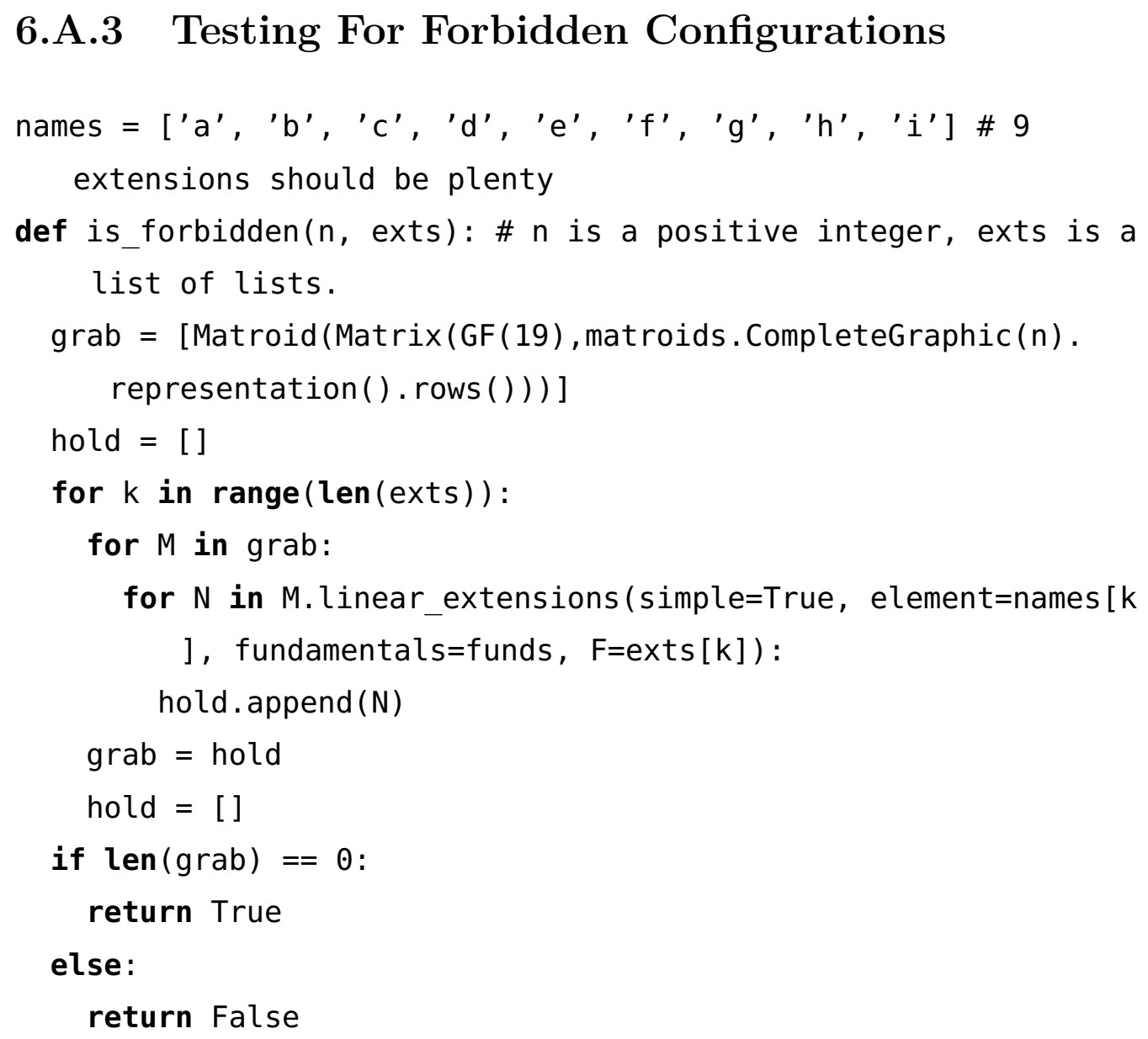

This code returns True if it is not possible to construct a golden-mean matroid by starting with $M\left(K_{\mathrm{n}}\right)$ and extending into all the flats in exts, and False otherwise.

\section{A.4 Finding Lines}

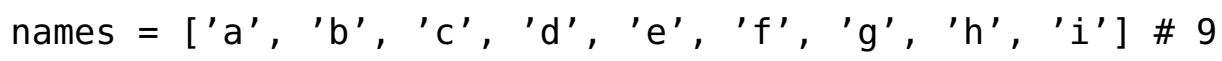

extensions should be plenty 


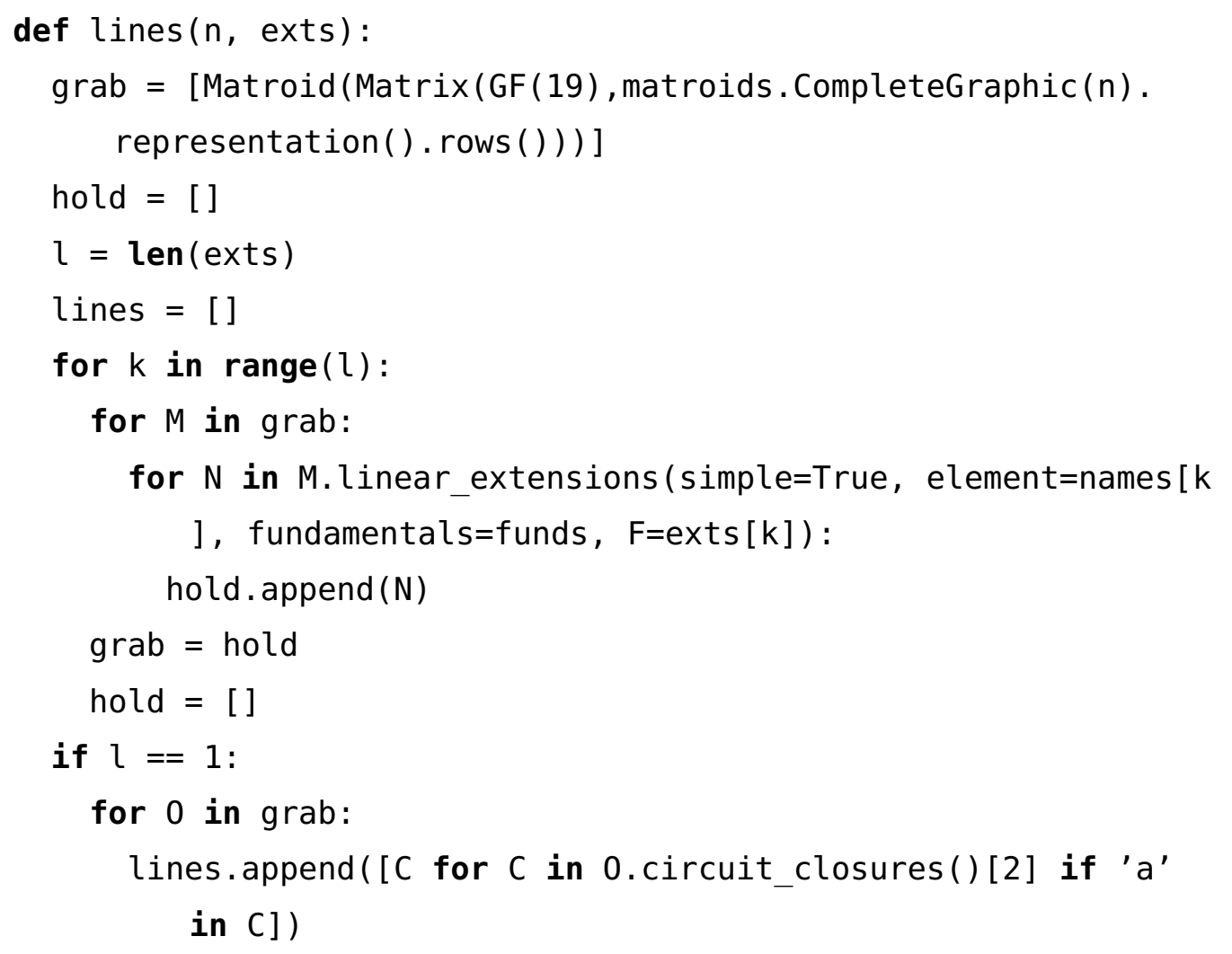

This code takes an augmented clique and constructs all possible golden-mean matroids that arise from that augmented clique. For each of the matroids constructed, lines returns the list of all lines that contain all of the extension 
elements.

\section{A.5 Finding Multiple Lines}

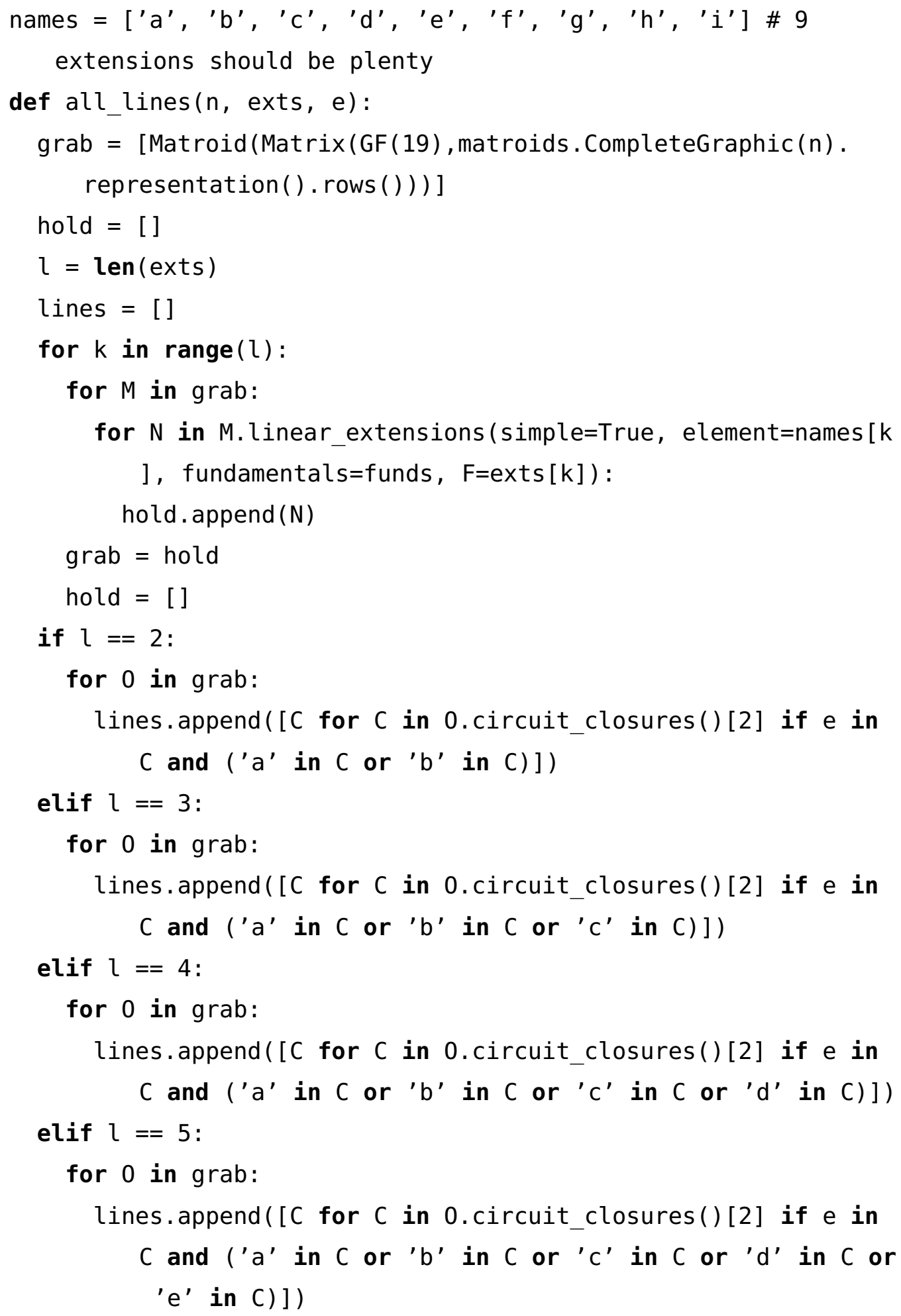




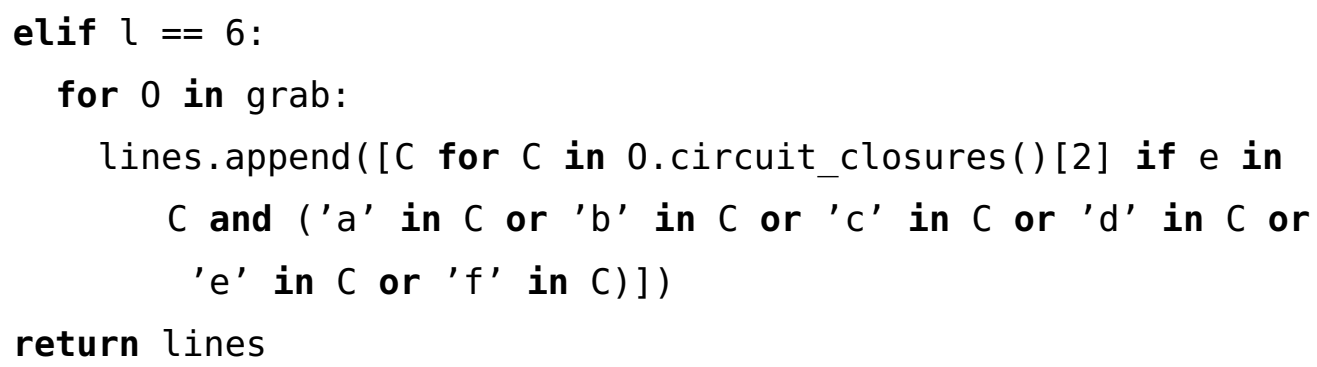

This code takes an augmented clique and constructs all possible golden-mean matroids that arise from that augmented clique. The function all_lines() then returns all the lines that contain a distinguished clique element e and at least one non-clique element. If it returns the empty list, then no matroids were constructed, and the configuration given is forbidden.

\section{A.6 Testing For Betsy Ross}

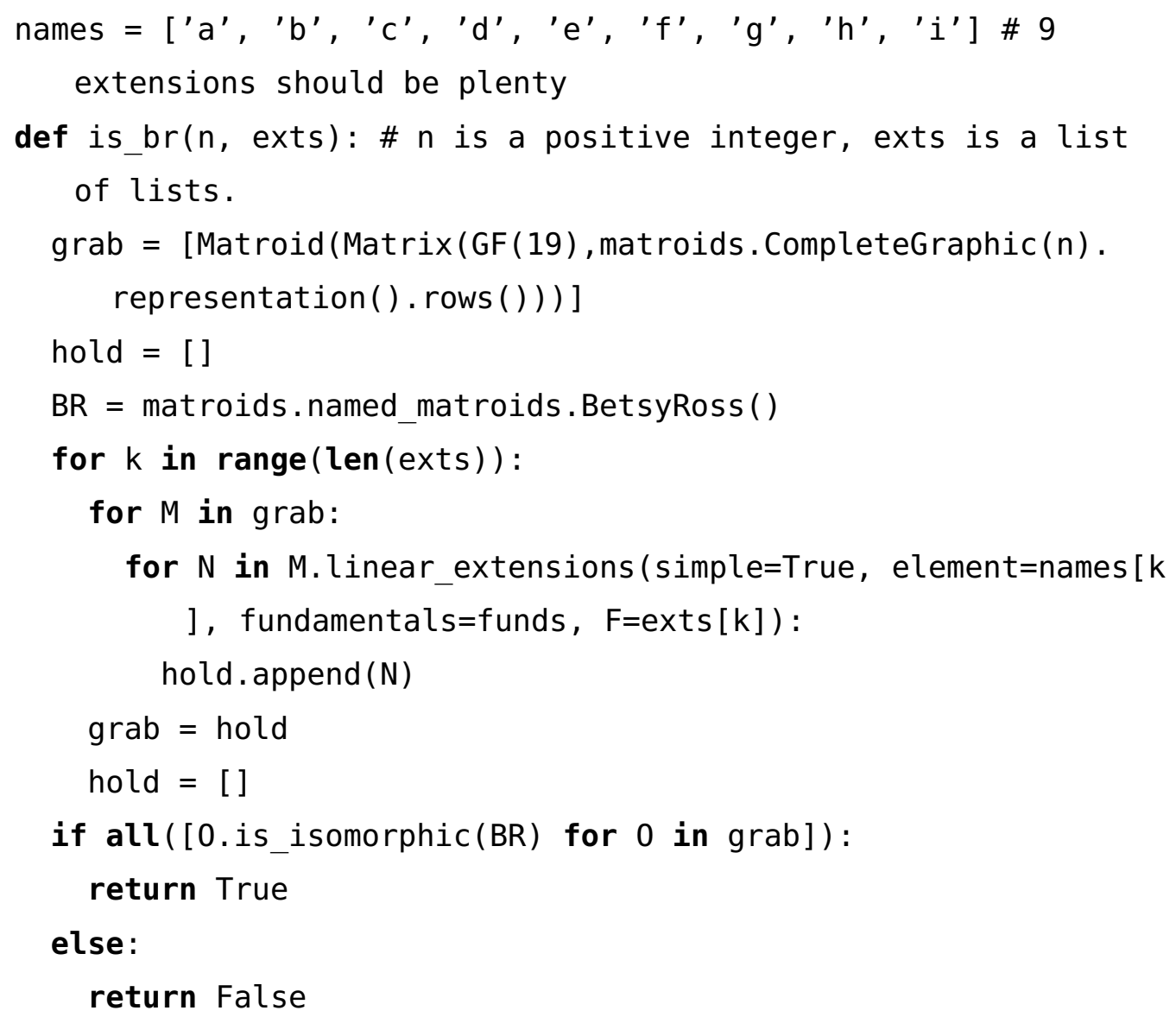

This code returns True if all matroids constructed by starting with $M\left(K_{\mathrm{n}}\right)$ and extending into all the flats in exts are isomorphic to the Betsy Ross 
(Figure 2.16), and False otherwise.

\section{A.7 Sublemma 6.6.19.1}

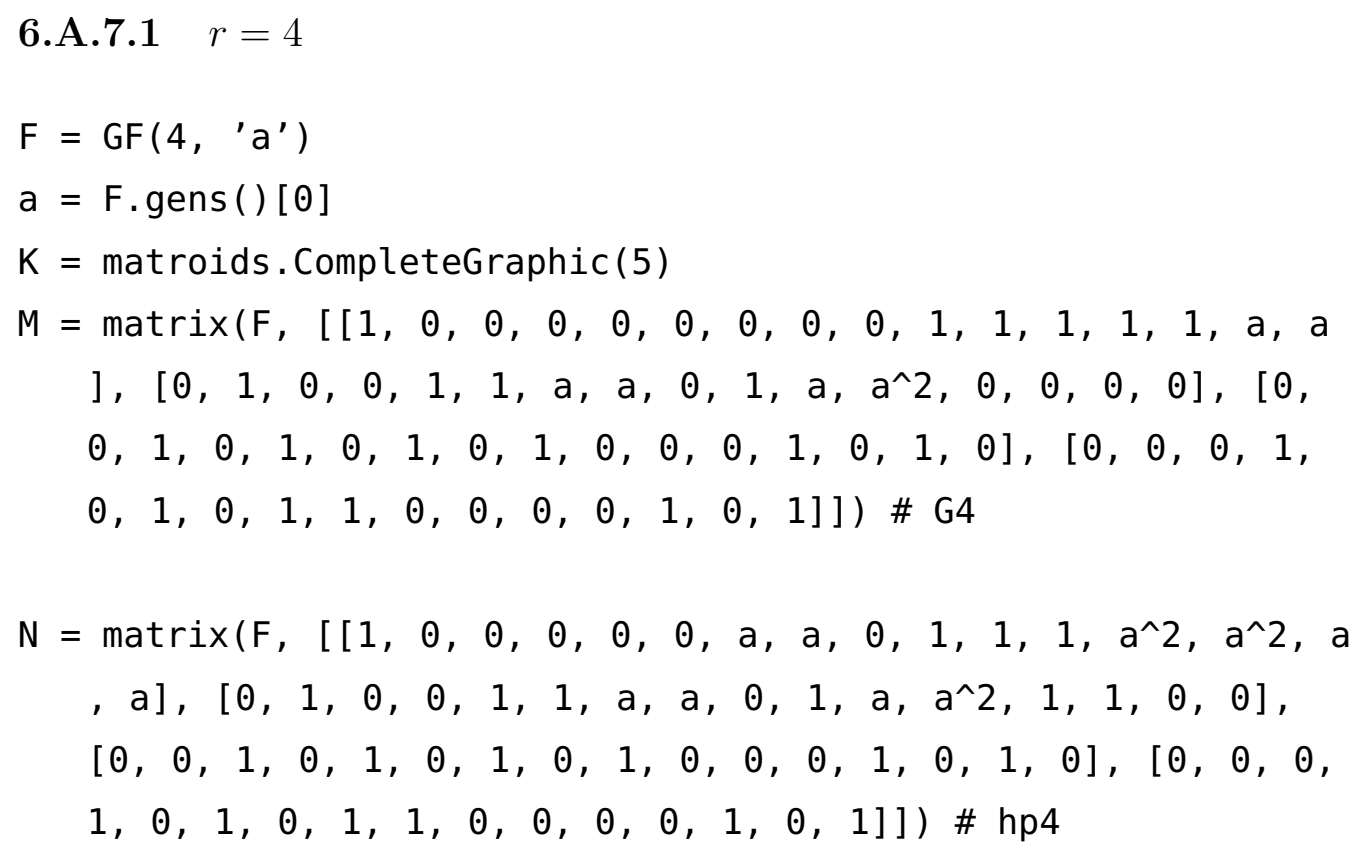




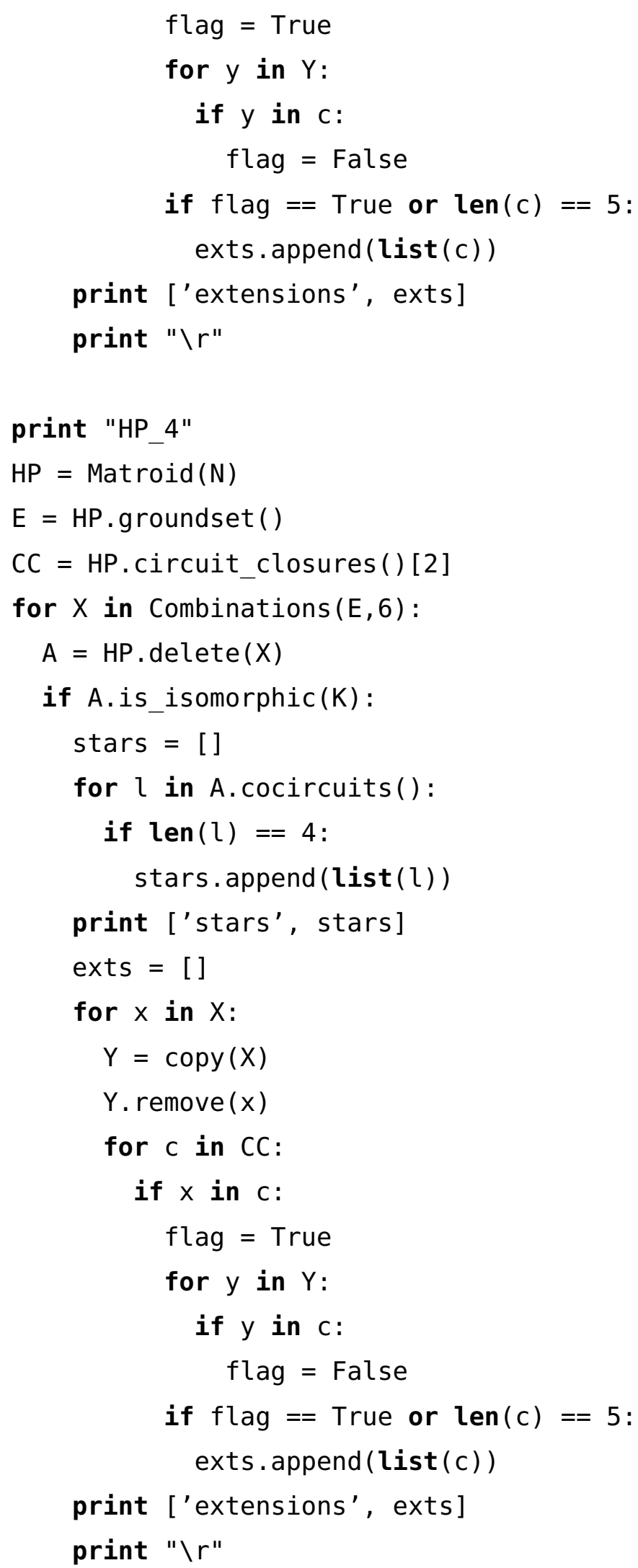




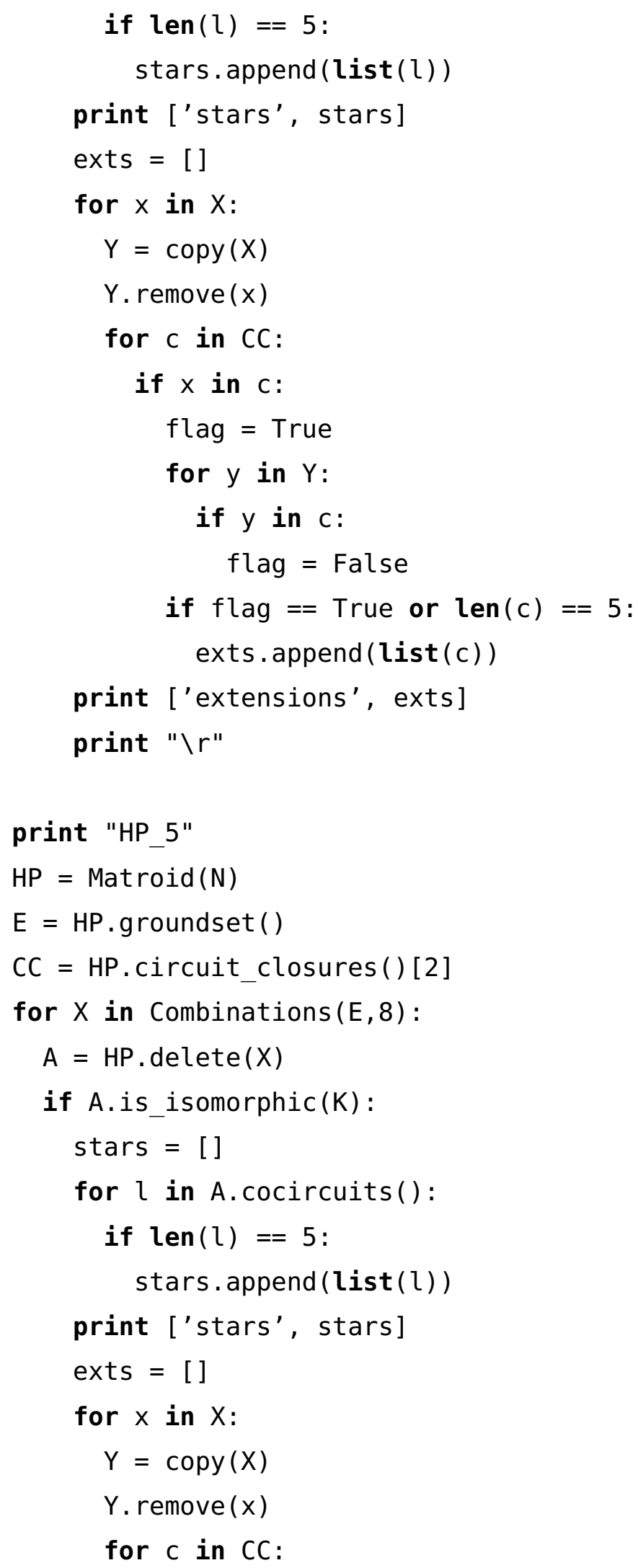




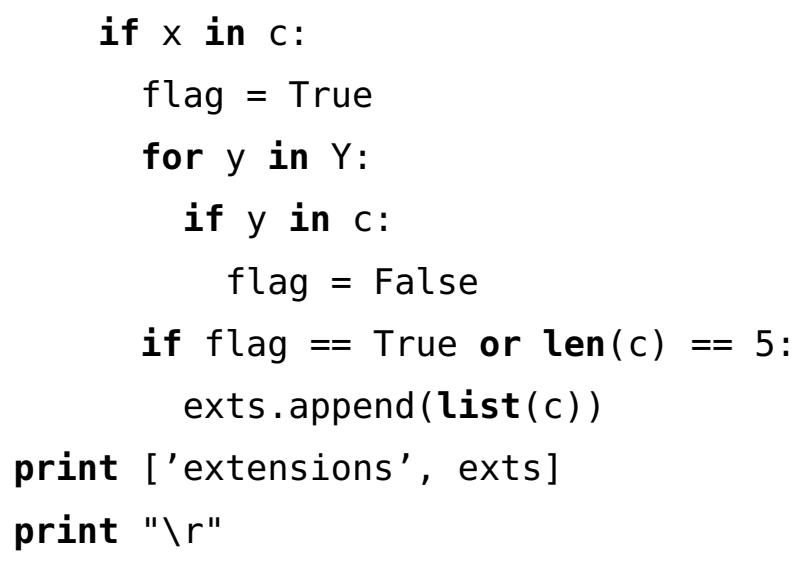

This code outputs, for each spanning clique $K$ in both $G_{5}$ and $H P_{5}$, two things. First, it outputs the stars in $K$, allowing us to create a labelled clique easily. It then outputs the rank-two flats that each non-clique element is contained in, allowing us to easily form the augmented clique. 


\section{Bibliography}

[1] Archer, S. Near Varieties and Extremal Matroids. PhD thesis, Victoria University of Wellington, 2005.

[2] Bixby, R. A simple theorem on 3-connectivity. Linear Algebra Appl. 45 (1982), 123-126.

[3] Diestel, R. Graph theory, fourth ed., vol. 173 of Graduate Texts in Mathematics. Springer, Heidelberg, 2010.

[4] Ding, G., Oporowski, B., Oxley, J., and Vertigan, D. Unavoidable minors of large 3-connected matroids. J. Combin. Theory Ser. B 71, 2 (1997), 244-293.

[5] Hall, R., Mayhew, D., and Van Zwam, S. The excluded minors for near-regular matroids. European J. Combin. 32, 6 (2011), 802-830.

[6] Heller, I. On linear systems with integral valued solutions. Pacific J. Math 7 (1957), 1351-1364.

[7] Kahn, J. On the uniqueness of matroid representations over $G F(4)$. Bull. London Math. Soc. 20, 1 (1988), 5-10.

[8] Kung, J. Combinatorial geometries representable over $G F(3)$ and $G F(q)$. I. The number of points. Discrete Comput. Geom. 5, 1 (1990), $83-95$.

[9] Kung, J., And Oxley, J. Combinatorial geometries representable over $G F(3)$ and $G F(q)$. II. Dowling geometries. Graphs Combin. 4, 4 (1988), 323-332. 
[10] Oxley, J. Matroid theory, second edition. Oxford University Press, USA, 2011.

[11] Oxley, J., Vertigan, D., and Whittle, G. On maximum-sized near-regular and $\sqrt[6]{1}$-matroids. Graphs and Combinatorics 14, 2 (1998), $163-179$.

[12] Pendavingh, R., And Van Zwam, S. Confinement of matroid representations to subsets of partial fields. J. Combin. Theory Ser. B 100, 6 (2010), 510-545.

[13] Pendavingh, R., And Van Zwam, S. Lifts of matroid representations over partial fields. Journal of Combinatorial Theory, Series B 100, 1 (2010), 36-67.

[14] Pendavingh, R., Van Zwam, S., et AL. Sage Matroid Package, included in Sage Mathematics Software 6.1.1. The Sage Matroid Development Team, 2014. http://www. sagemath . org.

[15] Semple, C. Matroid representation over partial fields. Master's thesis, Victoria University of Wellington, 1995.

[16] Semple, C. On maximum-sized k-regular matroids. Graphs Combin. 15, 4 (1999), 441-462.

[17] Semple, C., And Whittle, G. Partial fields and matroid representation. Adv. in Appl. Math. 17, 2 (1996), 184-208.

[18] Seymour, P. On minors of non-binary matroids. Combinatorica 1, 4 (1981), 387-394.

[19] Stein, W., ET AL. Sage Mathematics Software (Version 6.1.1). The Sage Development Team, 2014. http://www.sagemath.org.

[20] Van Zwam, S. Partial Fields in Matroid Theory. PhD thesis, Technische Universiteit Eindhoven, 2009.

[21] Welsh, M. Golden-mean and secret sharing matroids. Master's thesis, Victoria University of Wellington, 2011. 
[22] Whittle, G. A characterization of the matroids representable over GF(3) and the rationals. Journal of Combinatorial Theory, Series B 65, 2 (1995), 222-261.

[23] Whittle, G. On matroids representable over $G F(3)$ and other fields. Trans. Amer. Math. Soc. 349, 2 (1997), 579-603.

[24] Whittle, G. Recent work in matroid representation theory. Discrete Math. 302, 1-3 (2005), 285-296.

[25] Wu, Z. On the number of spikes over finite fields. Discrete Math. 265, 1-3 (2003), 261-296.

[26] Zaslavsky, T. Voltage-graphic matroids. In Matroid theory and its applications. Liguori, Naples, 1982, pp. 417-424.

[27] Zaslavsky, T. Biased graphs. II. The three matroids. J. Combin. Theory Ser. B 51, 1 (1991), 46-72. 
2-rounded, 35

$\alpha, 15$

augmented clique, 128

forbidden, 129

golden-mean, 129

green triangle, 128

red matching, 128

restriction, 128

balanced, 110

barbell graph, 110

$\beta, 4$

Betsy Ross configuration, 202

block, 111

e, $, 9,126$

clique

augmented, 128

spanning, 125

connected, 43

vertically $4-, 46$

core, 125

cross ratio, 13

cut vertex, 111

cycle

balanced, 110

$\mathbb{D}, 6$

deficit function

near-regular, 68 regular, 67

$d_{n}(N), 68$

$d_{r}(N), 67$

dyadic, 6

$\epsilon, 1$

exact vertical 3-separation, 46

extension

green, 128

red, 128

forbidden, 129

forbidden configuration, 129

function

growth-rate, 1

near-regular deficit, 68

regular deficit, 67

weight, 66

fundamental element, 12

$\mathbb{G}, 6$

$\mathscr{G}_{r}, 15$

golden-mean, 6

golden-mean augmented clique, 129

graph

balanced cycle, 110

barbell, 110

block, 111

cut vertex, 111

handcuff, 110 
star, 125

core, 125

theta, 110

green element, 128

green triangle, 128

growth-rate function, 1

$h(r), 7$

handcuff graph, 110

isomorphism

$\mathbb{P}$-matrix, 12

$k$-regular, 4

$\mathcal{L}(M, e), 63$

line, 9

length, 9

long, 9

non-clique, 125

very long, 9

line-star configuration, 193

semi-, 193

long line, 9

matching-star configuration, 198

maximal, 198

matroid

dyadic, 6

golden-mean, 7

$k$-connected, 43

$k$-regular, 4

$k$-separation, 43

exact, 43

near-regular, 4

$\mathbb{P}$-graphic, 109

partial field-representable, 3

regular, 3 sixth-roots-of-unity, 5

uses, 35

vertically 4-connected, 46

maximum-sized, 1

minor

$\mathbb{P}$-matrix, 12

$M\left(K_{2, b}^{+}\right)$

head, 63

limb, 63

$\mathcal{N}, 8,57$

$n$-spike, 58

standard form, 58

near-regular, 4

near-regular deficit function, 68

$\mathbb{P}^{*}, 3$

$\mathbb{P}$-graphic, 109

$\mathbb{P}$-matrix, 3

cross ratio, 13

isomorphism, 12

minor, 12

pivot, 12

scaling-equivalent, 12

$\mathbb{P}$-matroid, 3

partial field, 2

dyadic, 6

element, 3

fundamental element, 12

golden-mean, 6

homomorphism, 12

$k$-regular, 4

near-regular, 4

regular, 3

representable over, 3

sixth-roots-of-unity, 5

pivot, 12 
point, 9

$\mathcal{R}, 8,57$

red element, 128

red matching, 128

regular, 3

regular deficit function, 67

restriction, 128

RR-digon

parallel component, 147

path component, 147

$\mathbb{S}, 5$

scaling-equivalent, 12

semi-line-star configuration, 193

maximal, 193

semi-two-parallel configuration, 195

maximal, 196

separation, 43

exact, 43

exact vertical 3-, 46

sixth-roots-of-unity, 5

spanning clique, 125

spike

leg, 58

star, 125

core, 125

$T_{n}^{k}$

acme, 83

contour, 83

leg, 83

$\mathcal{T}$, 9, 109

$\tau, 7$

theta graph, 110

touch, 127

two-parallel configuration, 196 semi-, 195

$\mathbb{U}_{0}, 3$

$\mathbb{U}_{1}, 4$

$\mathbb{U}_{k}, 4$

uses, 35

vertically 4-connected, 46

very long line, 9

$w(N), 66$

weight function, 66 